\title{
Targeting the cerebrovasculature in sepsis: A focus on the brain microvascular endothelium
}

\author{
Divine C. Nwafor \\ West Virginia University, dnwafor@mix.wvu.edu
}

Follow this and additional works at: https://researchrepository.wvu.edu/etd

Part of the Behavioral Neurobiology Commons, Immunology of Infectious Disease Commons, Molecular and Cellular Neuroscience Commons, Nervous System Diseases Commons, and the Other Neuroscience and Neurobiology Commons

\section{Recommended Citation}

Nwafor, Divine C., "Targeting the cerebrovasculature in sepsis: A focus on the brain microvascular endothelium" (2021). Graduate Theses, Dissertations, and Problem Reports. 8340.

https://researchrepository.wvu.edu/etd/8340

This Dissertation is protected by copyright and/or related rights. It has been brought to you by the The Research Repository @ WVU with permission from the rights-holder(s). You are free to use this Dissertation in any way that is permitted by the copyright and related rights legislation that applies to your use. For other uses you must obtain permission from the rights-holder(s) directly, unless additional rights are indicated by a Creative Commons license in the record and/ or on the work itself. This Dissertation has been accepted for inclusion in WVU Graduate Theses, Dissertations, and Problem Reports collection by an authorized administrator of The Research Repository @ WVU.

For more information, please contact researchrepository@mail.wvu.edu. 


\title{
Targeting the cerebrovasculature in sepsis: A focus on the brain microvascular endothelium
}

\author{
Divine C. Nwafor \\ Dissertation submitted to the West Virginia University Health Science Center Neuroscience \\ Program in partial fulfillment of the requirements for the degree of
}

Doctor of Philosophy in Neuroscience

\author{
Randy J. Nelson, Ph.D., Chair \\ Mark R. Lee, M.D., Ph.D. \\ Bernard G. Schreurs, Ph.D. \\ Paul R. Lockman, Ph.D. \\ Candice M. Brown, Ph.D. \\ Department of Neuroscience, West Virginia University \\ Morgantown, West Virginia
}

2021

Keywords: Tissue-nonspecific alkaline phosphatase, Blood-brain barrier, Endothelial cells, Neuroinflammation, Sepsis, Alpl, Systemic inflammation, Cytoskeleton, Neuroimmune, BBB integrity 


\title{
ABSTRACT
}

\section{Targeting the cerebrovasculature in sepsis: A focus on the brain microvascular endothelium}

\author{
Divine C. Nwafor
}

The blood-brain barrier (BBB) is a critical interface between the systemic circulation and the brain. It is a specialized multicellular unit composed of brain microvascular endothelial cells (BMECs), pericytes, a basement membrane, and astrocytic end foot processes. BMECs are a principal component of the BBB that provide the structural framework needed for the stringent transport of molecules into the brain. BMEC dysfunction permits the trafficking of neurotoxins from systemic circulation into the brain, which ultimately exacerbates BBB dysfunction and neuroinflammation. Studies have shown that BBB dysfunction is a key determinant of cognitive decline in sepsis. However, there are critical knowledge gaps that exist in understanding the molecular and cellular mechanisms underpinning BMEC physiology and function as it relates to the maintenance of BBB integrity.

A strategy for bridging this critical knowledge gap requires an examination of proteins and enzymes localized to BMECs. Tissue-nonspecific alkaline phosphatase (TNAP) has been historically used as a brain endothelial histological marker due to its abundant expression on BMECs. Yet, the role of TNAP in BMECs remains unclear. The objective of this project was to understand the function of BMEC TNAP at the BBB in normal physiology and sepsis. Our central hypothesis is that BMEC TNAP is critical for maintenance of BBB integrity in normal physiology and in sepsis through molecular mechanisms that preserve BMEC structure and function. The studies presented in this dissertation demonstrated a novel antiinflammatory role for TNAP in cerebral microvessels. We also demonstrated that BMEC TNAP plays an important role in maintaining paracellular barrier integrity during systemic inflammation via cytoskeletal reorganization. Importantly, our data elucidated a molecular target (Rho- 
associated protein kinase) through which loss of TNAP activity in cerebral microvessel in sepsis could be mitigated. Finally, we utilized the cre-lox system to generate a genetic mouse model with an endothelial VE-cadherin conditional knockout of the Alp/ gene (VE-cKO). We showed that VE-cKO mice exhibited whole brain size selectively increased BBB permeability that is worsened in sepsis and stroke.

Collectively, this body of work demonstrated that TNAP activity in cerebral microvessel is important for maintaining BBB integrity. These findings lay the groundwork needed to stimulate the discovery of a therapeutic target whose activity can be manipulated to mitigate long-term neurological dysfunction in sepsis. Moreover, our results will impact not only sepsis but will improve the quality of life and medical outcomes in other neurodegenerative and inflammatory diseases such as stroke and Alzheimer's disease (AD). 


\section{Dedication}

This dissertation is dedicated to my parents, Peter and Patience Nwafor, whose unconditional love, encouragement, and support played an instrumental part in shaping who I am today. From them, I have learnt never to lose courage but continue my steadfast quest to discover the unknown. 


\section{Acknowledgement}

First and foremost, I would like to thank my mentor, Dr. Candice M. Brown, for her excellent mentorship and instruction which provided the foundation to start and complete this novel body of work. More importantly, your guidance and unwavering support empowered my development of autonomy and productivity.

Next, I must thank my committee members, Dr. Randy J. Nelson, Dr. Paul R. Lockman, Dr. Mark R. Lee, and Dr. Bernard G. Schreurs for their guidance, engagement, and expertise which greatly impacted the scientific work discussed in this dissertation.

I would like to thank my fellow lab compadres, Allison L. Brichacek, Dr. Sunjung Jun, Wei Wang, Dr. Sreeparna Chakraborty, and Sneha Gupta for their support with experiments and providing excellent feedback that improved the quality of the work discussed in this dissertation.

Finally, I must also thank my family and friends, Peter Nwafor, Patience Nwafor, Frank Nwafor, Nora-Anne Nwafor, Esther Nwafor, Nina Bidwai, Dr. Ashok Bidwai, Dr. Jyoti Bidwai, Dr. Benjamin Arah, Edith Arah, Emily Minor, and Shane Kaski, whose unconditional love and emotional support were instrumental in over-coming the numerous challenges I faced. 


\section{List of symbols, abbreviations and nomenclature}

5-((5-chloro-2-methoxyphenyl) sulfonamido) nicotinamide (SBI-425)

Accelerating rotarod (AR)

Adenosine triphosphate (ATP)

Adenosine diphosphate (ADP)

Adenosine monophosphate (AMP)

Aldosterone (ALD)

Alzheimer's disease (AD)

Alzheimer's disease related dementia's (ADRD)

Alkaline phosphatase (AP)

Amyotrophic lateral sclerosis

Analysis of variance (ANOVA)

Apparent diffusion coefficient (ADC)

Blood-brain barrier (BBB)

Bovine capillary endothelial cells (BCECs)

Breast cancer resistance protein (BCRP)

Brain microvascular endothelial cell (BMEC)

Cecal ligation and puncture (CLP)

Cerebral blood flow (CBF)

Cell index $(\mathrm{Cl})$

Centers of Biomedical Research Excellence (COBRE)

Central nervous system (CNS)

Choline acetyltransferase (ChAT

Circumventricular organs (CVOs)

Colon ascendens stent peritonitis model (CASP)

Corticosteroid (CORT)

Dephosphorylated LPS (dLPS)

Diffusion weighted imaging (DWI)

Dimethyl sulfoxide (DMSO)

Electroencephalogram (EEG)

Endothelial cell growth basal medium-2 (EBM-2)

Endothelial cell growth medium (ECGM)

Emergency Department (ED)

Gamma aminobutyric acid (GABA)

Gadolinium (Gd)

Germ cell alkaline phosphatase (GCAP)

Glial fibrillary acidic protein (GFAP)

Glucocorticoid receptors (GR)

Glucocorticoid response elements (GREs)

Glycosylphosphatidylinositol (GPI)

Green fluorescent protein (GFP)

Fetal bovine serum (FBS)

High mobility group box 1 protein (HMGBI)

Hot plate (HP)

Hour (h)

Human cerebral microvascular endothelial cell line (hCMEC/D3)

Human immunodeficiency virus (HIV)

Human recombinant AP (recAP)

Huntington's disease (HD)

Hypophosphatasia (HPP) 
Hypothalamic-pituitary-adrenal axis (HPA)

Hypoxanthine phosphoribosyltransferase (Hprt)

Intercellular adhesion molecule-1 (ICAM-1)

In-Cell Western (ICW)

IDeA Networks of Biomedical Research Excellence (INBRE)

Immunohistochemistry (IHC)

Immunoglobulin $\mathrm{G}(\mathrm{lgG})$

Institutional Development Awards (IDeA)

Interferon-gamma (IFN- $\gamma$ )

Interleukin (IL)

Intensive care unit (ICU)

Intestinal AP (IAP)

Leukoaraisosis (LA)

L-homoarginine (hArg)

Lipopolysaccharide (LPS)

Locus coeruleus (LC)

Lucifer Yellow $\left(\mathrm{Pe}^{\mathrm{LY}}\right)$

Magnetic resonance imaging (MRI)

Major facilitator super family domain containing 2a (Mfsd2a)

Medial vascular calcification (MVC)

Microglia/macrophage ionized calcium binding adaptor molecule 1 (lba-1)

Mineralocorticoid receptors (MR)

Minute ( $\min )$

Modified murine sepsis severity score (MMSS)

Monocyte chemoattractant protein-1 (MCP-1)

Monocytic myeloid-derived suppressor cells (M-MDSCs)

Mouse embryonic fibroblast (MEF)

Multiple sclerosis (MS)

Murine sepsis severity score (MSS)

Myeloid derived suppressor cells (MDSCs)

Myosin II regulatory light chain (MLC)

MLC phosphatase (MLCP)

National Institutes of Health $(\mathrm{NIH})$

Neurovascular unit (NVU)

Nucleus tractus solitarii (NTS)

Open field (OFT)

Parkinson's disease (PD)

Passive avoidance (PA)

Pathogen associated molecular patterns (PAMPs)

Paraformaldehyde (PFA)

Permanent Middle Cerebral Artery Occlusion (pMCAO)

Permeability glycoprotein (P-gp)

Phosphate $(\mathrm{Pi})$

Phosphate buffered saline (PBS)

Placental alkaline phosphatase (PLAP)

Polymorphonuclear myeloid-derived suppressor cells (PMN-MDSCs)

Primary Brain Microvascular Endothelial Cell (pBMEC)

Prostaglandin E2 (PGE2)

Pyrophosphate (PPi)

Radial arm water maze (RAWM)

Ras homolog family member A (RhoA) 
Recombinant human TNAP (rhTNAP)

Rho-associated protein kinase (ROCK)

Seconds (s)

Sepsis-associated encephalopathy (SAE)

Sphingosine-1-receptor-1 (S1Pr1)

Subcutaneously (s.c.)

Tight junctions (TJs)

Tissue-nonspecific alkaline phosphatase (TNAP)

Tissue-nonspecific alkaline phosphatase inhibitor (TNAPi/TNAPI)

Toll-like receptor-4 (TLR4)

Transendothelial electrical resistance (TEER)

Transient Middle Cerebral Artery Occlusion (tMCAO)

T-regulatory (Tregs)

Tumor necrosis factor alpha (TNF- $\alpha$ )

Tumor necrosis factor receptor 1 (TNFR1)

Tumor necrosis factor receptor 2 (TNFR2)

Two-day radial arm water maze (2D-RAWM)

Wild-type (WT)

Vagotomy (VGX)

Vagus nerve (VN)

von Willebrand Factor (VWF) 


\section{TABLE OF CONTENTS}

\section{Chapter 1. Introduction}

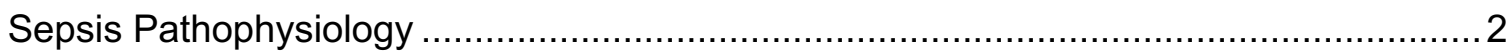

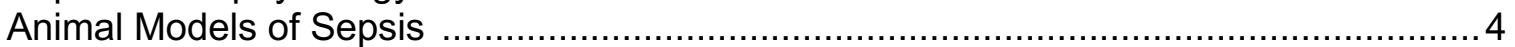

Blood-Brain Barrier (BBB) Dysfunction in Sepsis ...................................................10

Tissue-nonspecific Alkaline Phosphatase (TNAP) at the BBB ................................... 14

Gaps in Knowledge and Project Objectives .......................................................... 19

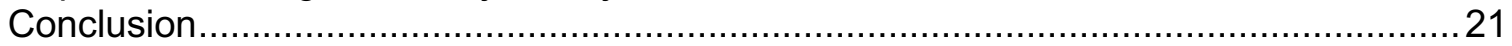

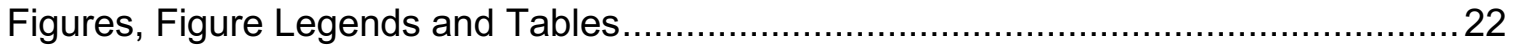

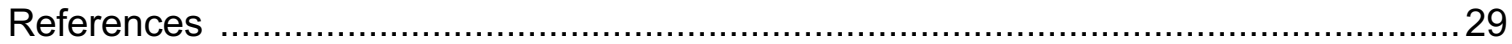

Chapter 2. Loss of tissue-nonspecific alkaline phosphatase (TNAP) enzyme activity in cerebral microvessels is coupled to persistent neuroinflammation and behavioral deficits in late sepsis

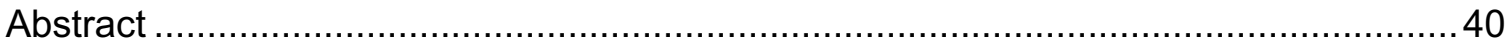

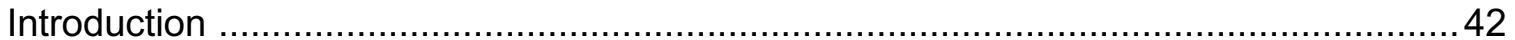

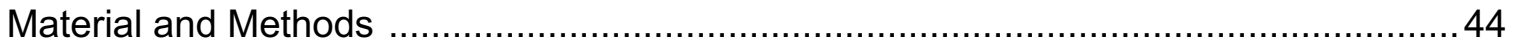

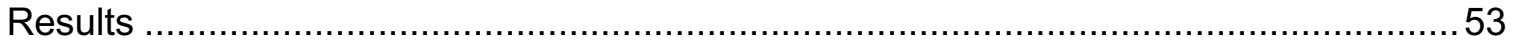

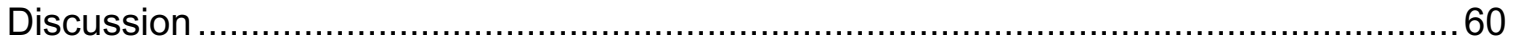

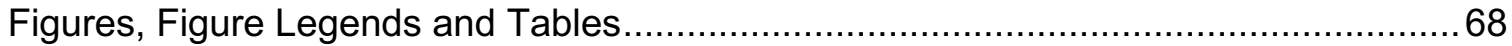

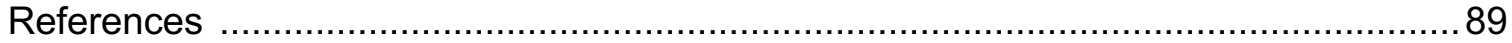

Chapter 3. Brain endothelial cell tissue-nonspecific alkaline phosphatase (TNAP) activity promotes maintenance of barrier integrity via the ROCK pathway

Abstract $\begin{aligned} & \text { Introduction } \ldots \ldots \ldots \\ & \text { Material and Methods } \\ & \text { Results } \\ & \text { Discussion }\end{aligned}$

Chapter 4. A Brief Report: The in vivo effects of a conditional loss of brain endothelial cell tissue-nonspecific alkaline phosphatase (TNAP) on blood-brain barrier permeability in the early stages of injury

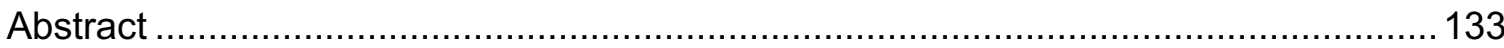

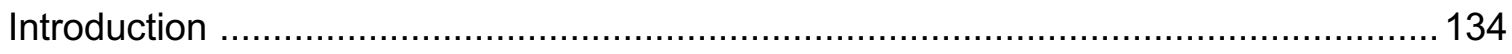

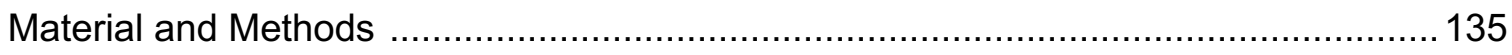

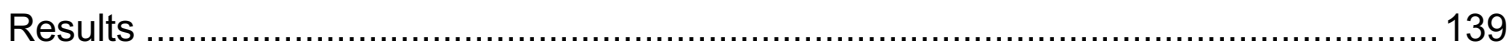

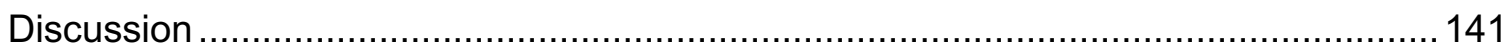

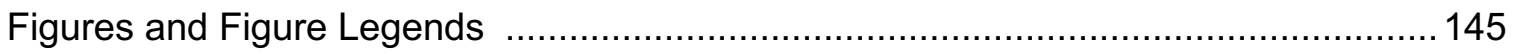

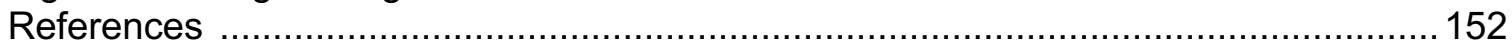

\section{Chapter 5. Discussion}

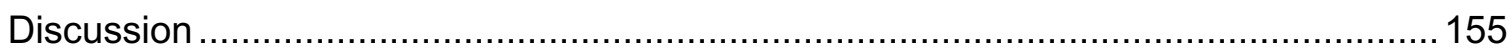


A Novel Anti-Inflammatory Role for TNAP in Cerebral Microvessels ......................... 156

TNAP-mediated signaling pathways in brain endothelium ...................................... 160

Deciphering the role of BMEC TNAP at the neurovascular unit in vivo....................... 162

Restoring AP activity as therapy to treat neuroinflammatory diseases .......................166

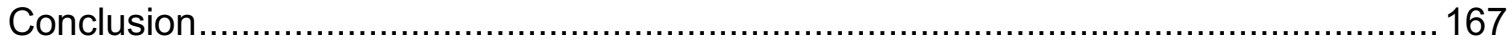

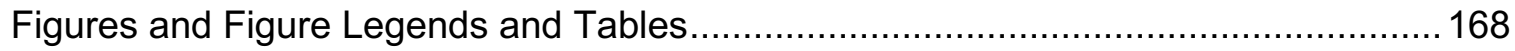

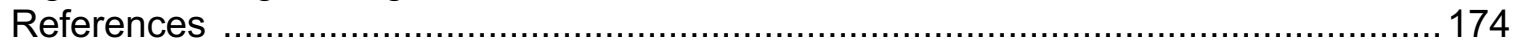




\section{Chapter 1}

\section{Introduction and literature review}

Nwafor DC, Brichacek AL, Mohammad AS, Griffith J, Lucke-Wold BP, Benkovic SA, Geldenhuys WJ, Lockman PR, Brown CM. Targeting the Blood-Brain Barrier to Prevent SepsisAssociated Cognitive Impairment. J Cent Nerv Syst Dis. 2019 Apr 9; 11:1179573519840652. doi: 10.1177/1179573519840652. PMID: 31007531; PMCID: PMC6456845.

Nwafor, D.C, Brichacek, A.L, Chakraborty, S., Gambill, C., Benkovic, S., \& Brown, C.M. (2020, March 31). Neuroendocrine and Neuroimmune Mechanisms Regulating the BloodBrain Barrier. Oxford Research Encyclopedia of Neuroscience. doi: 10.1093/acrefore/9780190264086.013.307

Nwafor, DC., Brichacek, AL., Ahsan, A., \& Brown, CM. TNAP in Central Nervous System Health and Disease: A Focus on Brain Microvascular Endothelial Cells. International Journal of Molecular Sciences (in press)

Note: Parts of this chapter include text and figures taken from the publications highlighted above 


\section{Background}

Sepsis is a debilitating systemic inflammatory process involving multiple organ systems that is preceded by an infection. It is the $10^{\text {th }}$ leading cause of death in the United States with an annual financial burden for patients and survivors that exceeds $\$ 20$ billion [1, 2]. Through mechanisms that remain largely poorly understood, sepsis can induce acute and chronic changes in the central nervous system (CNS), particularly at the blood-brain barrier (BBB) [3]. A compromised CNS can lead to sepsis-associated encephalopathy (SAE), a well-characterized state of cognitive impairment and neurological dysfunction often seen in the acute phase of sepsis. Multiple pathways have been investigated for their contribution to the sepsis-associated compromise of the BBB. This chapter integrates current clinical knowledge of sepsis with mechanistic insights from both clinical studies and preclinical animal models of sepsis. The overall goal of this chapter is to demonstrate how sepsis pathophysiology perturbs the integral function of cells and proteins that comprise the BBB. We provide insights to uncover how a compromised BBB may lead to SAE or permanent brain dysfunction in sepsis survivors. Finally, we discuss how tissue-nonspecific alkaline phosphatase (TNAP), an enzyme localized to brain microvascular endothelial cells (BMECs), may play an important role in maintaining endothelial barrier integrity.

\section{Sepsis Pathophysiology}

\section{A. Clinical sepsis presentation}

The most current definition from the Sepsis-3 consortium describes sepsis as a lifethreatening organ dysfunction caused by a dysregulated host response to an infection [4]. The most common precipitating sites for sepsis are the respiratory system, genitourinary system, and the abdomen $[5,6]$. Sepsis may present as a combination of various non-descript signs and symptoms making early diagnosis difficult. For example, patients may present with fever, chills, pain, delirium, increased heart rate, shortness of breath, diarrhea, and/or low blood pressure. 
The diagnosis and management of sepsis has changed dramatically over thirty years. The historical definition of sepsis was focused primarily on inflammation and incorrectly portrayed sepsis as a sequential process that eventually ends in septic shock (Fig 1A). In 2016, Sepsis-2 criteria were revised to current Sespis-3 criteria to improve consistency in classification in epidemiological and clinical trials. The revised Sepsis-3 classification shown in Fig. 1B focuses on accelerated recognition and management of sepsis [4].

In recent years the focus in clinical treatment has shifted to severe sepsis and septic shock, which has increased survival in hospitalized patients diagnosed with severe sepsis and critically ill patients with septic shock who have a higher risk of multi-organ failure complications and death [5]. This heterogeneous presentation of clinical sepsis makes disease management and appropriate therapeutic interventions difficult. Some challenges associated with the management of sepsis include late diagnoses, poor prognoses, inadequate therapeutics, and post-sepsis complications. These challenges stem from late recognition and difficulties associated with differentiation of sepsis from other illnesses in its early stage. In the later stages of sepsis, recognition becomes easier, yet sepsis is more difficult to treat and often coincides with multi-organ failure $[5,7,8]$.

Most sepsis cases are hospital-acquired. Hospital-acquired sepsis cases are often comorbid with prior injury, such as stroke, trauma, or post-surgery. Most cases of hospitalacquired sepsis are treated in the intensive care unit (ICU). However, ICU heterogeneity can make sepsis more common in one ICU versus another. For example, there is a higher incidence of sepsis in a trauma ICU as opposed to a surgical ICU [5]. Alternatively, a significant number of sepsis patients are admitted to the hospital or directly to the ICU via the Emergency Department [9]; the majority of these patients present with community-acquired sepsis from pneumonia or complications from other comorbid conditions such as diabetes $[10,11]$. In addition to the patient setting, the development of sepsis often depends on several risk factors, such as age where a proportionate relationship exists between increasing age and sepsis acquisition [12, 
13]. Male gender, non-Caucasian ethnicity, and pre-existing conditions such as Alzheimer's disease (AD), human immunodeficiency virus, or cancer are also risk factors for sepsis acquisition [5, 13-15].

\section{B. Current experimental animal models of sepsis}

Understanding the mechanisms involved in the pathophysiology of sepsis requires the use of animal models that adequately reproduce several features of the human disorder including both inflammation and infection. The most common animal models are: cecal ligation and puncture (CLP), the colon ascendens stent peritonitis model (CASP), endotoxin injection, and bacterial infusion [16]. The CLP model is regarded as the gold standard for human-like sepsis progression in animal models [17]. Execution of this model necessitates leakage of polymicrobial feces into the peritoneum after the cecum is punctured with a needle. Animals typically show decreased cerebrovascular blood flow post-CLP surgery and disease severity is modeled by controlling needle size and number of punctures (Fig. 2); however, a major limitation of this model is the failure to maintain continuous fecal leakage due to abscess formation and necrosis of the punctured bowel. Some investigators also administer antibiotics either at the time of injury or at intervals post-injury. Although antibiotic administration is an additional feature which mimics the treatment regimen in human patients, the use of different antibiotic classes and dosing paradigms across laboratories may confound the interpretation of findings when results are compared between laboratories $[16,18]$. The CASP model is a newer model recently introduced to counter the flaws of the CLP model $[16,19]$. This model involves the insertion of a stent at the ascending portion of the colon, allowing continual leakage of feces into the peritoneum $[16,20]$. Despite the resemblance to human-like sepsis progression, the drawbacks to this model include animal variation in colon size, fecal content, and the volume of feces that leaks into the peritoneum [16, 21]. Although these two models have provided 
remarkable insights to understanding the pathophysiology of sepsis, they fail to fully recapitulate the comprehensive clinical progression of sepsis in humans [16].

Two alternative sepsis models involve injection or infusion of endotoxin or bacteria. The endotoxin model typically involves injection of lipopolysaccharide (LPS) endotoxin, a component of gram-negative bacterial cell walls which signals most commonly through toll-like receptor-4 (TLR4). Administration of LPS via different routes (i.e., intraperitoneal, intravenous, or intracerebroventricular) initiates a cytokine storm that results in the release of tumor necrosis factor alpha (TNF- $\alpha$ ) and numerous Interleukins (IL-1, -6, and -10). Injection of LPS mimics many classical signs and symptoms of sepsis-induced inflammation, thereby providing a basic understanding of how inflammation activates the immune response in sepsis [16, 22]. One major limitation of the LPS model is the lack of integration of the infection component. A second limitation is that very large endotoxin doses are required in many rodent models to mimic the pathological profile of the clinical sepsis picture observed in humans [23]. Bacterial injection is a less widely used model involving infusion of a bacteria (usually Escherichia coli or Staphylococcus aureus) to initiate both inflammation and infection [16, 24, 25]. Different bacterial strains used for infection present a challenge in this model, as they will produce different patterns of sepsis progression [26]. Thus, the characteristics of the sepsis model must be considered when interpreting the effects of sepsis on the CNS and other organ systems. The CLP model was utilized for a majority of the experiments discussed in this dissertation.

\section{The CNS in sepsis: sickness behavior and sepsis-associated encephalopathy (SAE)}

A critical role for the CNS in the pathophysiology of sepsis has emerged over the past two decades. Several recent reviews address this topic in excellent detail $[13,27-30]$. One important contribution of the CNS is "sickness behavior". Sickness behavior is a response seen in sepsis characterized by fever, adaptive behavioral changes, and neuroimmune changes [31]. The response is governed primarily by systemic interactions with the vagus nerve (VN) and 
circumventricular organs (CVOs). The VN is an important mediator of inflammation. Septic mice that underwent a vagotomy (VGX) surgery exhibited an increase in the synthesis of inflammatory cytokines compared to sepsis only mice [27, 32-34]. In contrast, stimulation of the VN in septic animals resulted in an overall reduction in the synthesis of inflammatory cytokines, leukocyte recruitment, and endothelial activation [34-36]. The VN also relays peripheral information to the medullary autonomic nuclei while the CVOs allow passage of inflammatory mediators, primarily cytokines, from the peripheral circulation into the brain parenchyma. These inflammatory mediators activate the nucleus tractus solitarii (NTS) and locus coeruleus [37]. Activation of these brainstem areas relay signals to autonomic nuclei, behavioral, and neuroendocrine centers [38, 39]. The summative effect can be observed as depression, social withdrawal, increased heart rate, poor blood pressure control, or altered vigilance [18].

In addition to sickness behavior, patients with acute sepsis may have changes in brain function that present as delirium, seizures, psychological disorders, abnormal motor movements, and increased mortality $[38,40]$. Changes in brain function are most commonly manifested as delirium. While sepsis-associated delirium usually presents as decreased activity, a hyperactive form associated with agitation may be seen in some patients [38]. Tools that can be utilized to confirm sepsis-associated delirium include medical history, blood chemistry, electrolyte balance, the ICU screening checklist, Confusion Assessment Method, and Glasgow Coma Scale $[38,41]$. Sickness behavior and/or delirium may progress to a more severe phenotype, sepsis associated encephalopathy (SAE), which is regarded as a diagnosis of exclusion [42]. It is characterized by impaired consciousness, seizures, delirium, coma, focal cognitive deficits and alterations in electroencephalogram [43] patterns [44]. Patients with SAE have increased mortality, long-term neurological decline, memory lapse, inattentiveness, disorientation, and verbal difficulties [45].

Alterations in EEG wave patterns often predict SAE outcome, and EEG reactivity is associated with mortality even at one-year post-severe sepsis [44, 46]. For example, a recent 
study showed resting-state EEG changes in sepsis survivors at 6-24 months after hospital discharge, including increased delta and sigma activity compared to control patients [47]. Changes in EEG frequencies can be associated with changes in brain function. For example, slowing alpha activity with increased theta activity reflects cortical dysfunction and can occur in patients with mild to moderate encephalopathy. Slowing of delta activity is often associated with more severe neurocognitive decline and indicates impaired function in deeper brain structures, such as the basal ganglia [44]. While evaluation of EEG can be sensitive for SAE diagnosis in the absence of neurological examination abnormalities, it has poor specificity and can be hampered by sedation and analgesia $[45,48]$.

Ischemia is another common complication of early sepsis due to drastic changes in systemic blood pressure [40]. A number of human clinical studies support the premise of decreased cerebral blood flow (CBF) in acute sepsis [49-52]. The abrupt change in blood pressure with added sepsis-associated coagulopathy causes reduced blood flow to neurons. The hippocampal region and watershed areas are affected more often than other brain regions when this occurs. Autopsies in patients who died from septic shock revealed consistent ischemic and hypoxic insults in areas particularly susceptible to low blood flow (e.g. amygdala, frontal junctional cortex etc.) and in autonomic centers [53]. Furthermore, the autopsies of seven delirious ICU patients in another study revealed pathological lesions in the hippocampus, striatum, and pons triggered by ischemia or hypoxia [54]. The presence of ischemia in postmortem studies strongly suggest that vascular irregularities and alterations of CBF occur during sepsis. Importantly, multiple clinical observations support the concept that, in the absence of cerebrovascular occlusion (i.e., stroke), impaired cerebral autoregulation and hypotension may be the primary drivers of tissue hypoxia and cerebral ischemia observed in sepsis patients [5557].

A recent study in rats conducted by Towner et al. showed that CBF in the thalamus and cortex is significantly increased 24 hours post-LPS injection but significantly reduced six weeks 
post-LPS injection when compared to saline controls [58]. Preceding human clinical studies also support the decrease in CBF at 24 hours observed by Towner and colleagues, yet there remains a paucity of literature on how sepsis may affect long-term CBF in human patients. Overall, coincident alterations in cerebral blood and cardiovascular collapse in sepsis emphasize the importance of fluid resuscitation as a crucial component of sepsis management. The ideal type of fluid (colloid versus crystalloids) and ideal composition used to treat septic patients remains controversial. Three clinical trials demonstrated that colloid use in sepsis treatment failed to show a clear benefit [59-61]. In addition to the findings from this study, the restricted accessibility, safety issues, and the expense of colloids shifted the debate towards identifying the ideal crystalloid composition (e.g. Ringer's lactate, Ringer acetate etc.) [62]. We refer the reader to excellent reviews regarding optimal fluid therapeutic strategies in sepsis [6264].

The utilization of magnetic resonance imaging (MRI) in the diagnosis of SAE offers a unique opportunity in capturing some of the morphologic, ischemic, and metabolic alterations associated with sepsis. A summary of MRI findings in acute sepsis is shown in Table 1. In particular, diffusion weighted imaging (DWI) and the apparent diffusion coefficient (ADC) are two MRI modalities currently used in assessing BBB breakdown caused by vasogenic (extracellular) or cytotoxic (intracellular) edema [65]. Cytotoxic edema typically caused by ischemia, hypoxia, or vasogenic edema is the most consistently reported MRI change associated with SAE [65-68]. Early detection of BBB breakdown by gadolinium (Gd) could establish an adequate therapeutic window for current and future septic treatments, but human studies are limited [69-71]. A recent study in rats revealed a significant increase in the infiltration of $\mathrm{Gd}$ in the cortex, hippocampus, and thalamus 24 hours and 1-week post LPS injection [58]. Gd use may also cause a substantial risk of nephrogenic systemic fibrosis, a risk factor which suggests that Gd-based imaging in the CNS should be evaluated on a case-by-case basis. 
Sedatives are often administered in the ICU when treating sepsis. A study conducted by Qiao and colleagues in rats showed that the application dexmedetomidine and midazolam improved survival, and reduced cytokine levels and splenic apoptosis in septic mice [72]. A systematic review by Zamani et al. emphasized the importance of the kind of sedative used in treating sepsis; the findings from this study revealed that dexmedetomidine improved short term mortality when compared to other sedatives [73]. It is widely thought that the neuroprotective effects of dexmedetomidine result from neuronal death prevention, suppression of inflammatory cytokines, and the modulation of neurotransmitters released in the sympathetic nervous system [73-76]. However, a limitation noted by Zamani et al. was the small sample size included in the clinical studies. It is important to note that the tools used in the confirmation of sepsisassociated delirium are not helpful in ICU-sedated patients who may otherwise exhibit signs of delirium [42].

Sepsis also affects long-term neurological outcomes. The greatest risk factor for longterm impairment is the duration of delirium in the acute phase of sepsis and the increased ventricular to brain ratio as calculated by $\operatorname{MRI}[38,40,77,78]$. A seminal study published by Iwashyna and colleagues suggested that up to $70 \%$ of sepsis survivors may exhibit lasting neurological impairment, including alterations in mood, cognition, and motor function [79]. Cognitive, motor, and mood impairments are three of the most common long-term neurological outcomes in septic patients [40]. Current evidence also suggests an increased susceptibility to other neurodegenerative disorders such as stroke or AD post-sepsis insult [80]. Thus, patient populations that are the most vulnerable to long-term neurologic decline post-sepsis are the elderly and patients with preexisting neurodegenerative diseases $[12,13,38,80]$. The consequences of sepsis on both acute and chronic neurological outcomes demonstrates a critical need to understand the mechanisms involved in SAE development. Harnessing this knowledge will provide essential therapeutic avenues to limit SAE progression and protect against long-term neurological impairment or dysfunction. 


\section{Mechanisms of Blood-Brain Barrier Dysfunction in Sepsis}

\section{A. Blood-brain barrier overview}

This section will provide a brief overview of the cell biology and physiology of the BBB. The BBB and other associated cell types within the neurovascular unit (NVU) are shown in Figure $3[38,81]$. The BBB is a highly-selective, dynamic, and semipermeable biological interface between the brain parenchyma and cerebral circulation. Preservation of BBB integrity protects normal brain function and is dependent on maintaining a precise cerebral homeostasis driven, in large part, by ion and gas concentrations and nutrient availability. The BBB's unique structure is comprised of endothelial cells, astrocytes, pericytes, and a basal lamina. The endothelial cells are joined by tight junctions (TJs), and surrounding pericytes and astrocytes, which associate with the basal lamina to protect the brain parenchyma. Endothelial cells are sealed or joined together by TJs which are comprised of occludin, claudin, and cadherin proteins. Because paracellular movement of compounds around and between endothelial cells is highly restricted, active transport is required to move polar solutes and nutrients across the BBB into the brain parenchyma [82]. However, water, small gases, and small- to moderatelysized lipid-soluble compounds can enter the brain passively [83, 84].

Primary efflux transporters include permeability glycoprotein (P-gp) and breast cancer resistance protein (BCRP), which actively pump compounds out of the brain and back into circulation [82]. Often, these transporters further restrict the permeability of compounds and drugs which may otherwise have the molecular characteristics to passively cross the BBB [85]. The concept of the NVU emphasizes the continual crosstalk and interactions between the BBB, other resident CNS cells such as neurons, microglia, and oligodendrocytes, and peripheral immune cells. The BBB-NVU crosstalk also forms a network in which all cells are under constant surveillance and adapt their behavior in both healthy and disease states [86]. Readers 
are referred to these excellent in-depth reviews about the function and structure of the BBB and NVU [83, 86-88].

B. Neuroinflammation, immune cell infiltration, and BBB permeability post-sepsis: $A$ focus brain microvascular endothelial cells (BMECs)

BMECs are a principal component of the BBB that provide the structural framework needed for the stringent transportation of molecules into the brain, and maintenance of precise cerebral homeostasis. A probable starting point of sepsis-induced brain dysfunction is the initiation of brain endothelial cell damage followed by chronic neuroinflammation, but the mechanism by which this occurs is not well understood [51]. Neuroinflammation is a response to CNS disruption or dysfunction and it is typically found in many neurological disorders [89]. Current literature suggests neuroinflammation in sepsis begins when immune cells recognize foreign pathogen associated molecular patterns (PAMPs) such as LPS, flagellin, fimbriae, peptidoglycan, heat shock proteins, and DNA fragments, which are encoded as 'danger signals' to the host. Recognition of PAMPs causes release of pro-inflammatory cytokines in the periphery [90]. Inflammatory mediators may enter the brain by numerous mechanisms that include: transcellular diffusion, solute carrier proteins, receptor-mediated transcytosis, and adsorptive transcytosis [91]. Many cytokines enter the brain through receptor-mediated endocytosis on brain endothelial cells. For example, during inflammation TNF- $\alpha$ is upregulated and its transportation from blood to brain parenchyma is increased, primarily through receptormediated endocytosis of its receptors, tumor necrosis factor receptor 1 (TNFR1) and tumor necrosis factor receptor 2 (TNFR2) [92, 93]. Molecules originating in peripheral or CNS tissues may activate the brain vascular endothelium and various leukocytes to produce hormones that facilitate their entry into the brain. For example, Nishijima and colleagues observed that prostaglandin E2 (PGE2) enhanced transport of serum insulin-like growth factor 1 across the BBB [94]. 
Cytokine production contributes to neuronal dysfunction in sepsis in addition to many other neurological disorders. Cytokine infiltration enhances activation of brain endothelial cells and microglia, which ultimately leads to loss of neuronal function. Activation of the brain endothelium leads to enhanced activity of the coagulation cascade, microthrombi formation, and ischemia, which, in turn, promotes increased BBB permeability, leukocyte infiltration and neuroinflammation. This process triggers neuronal damage, apoptosis, and brain edema [95, 96]. Cytokine-mediated microglial activation occurs simultaneously with brain endothelial cell activation. Although the normal microglial response is to phagocytose injured neuronal cells and clear debris, sustained and dysregulated microglial activation is highly detrimental to specific regions of the CNS. Thus, persistent microglial activation enhances the production of inflammatory cytokines and reactive oxygen species (ROS), which perpetuates a vicious cycle of increased BBB permeability coupled with neuronal damage and apoptosis [92, 95]. Collectively, neuronal apoptosis and microglial activation are two primary mechanisms that increase the activity of inducible nitric oxide synthase (iNOS) activity and generation of nitric oxide (NO). Neuronal apoptosis is further exacerbated due to neuron sensitivity from increased levels of NO produced by activated microglia [97, 98]. Intriguingly, iNOS levels are elevated in sepsis and are highest in deceased septic patients [99]. This increased iNOS activity could also be responsible for the cardiovascular collapse seen in sepsis $[53,93,99]$. It is likely that this cardiovascular collapse also affects the cerebral microcirculation and leads to subsequent sepsis-associated brain dysfunction.

At the onset of inflammation, peripheral leukocytes migrate through the tightly regulated BBB and enter myriad areas of the CNS including but not restricted to CSF, CP, meninges, perivascular spaces, and eventually into cerebral parenchymal tissues [89]. Transcellular migration of leukocytes across the BBB during inflammation is a multi-step process. These steps are: (i) rolling: weak adhesions between brain endothelial cell selectins and leukocytes (e.g. neutrophils, monocytes, macrophages etc.); (ii) activation: G-coupled receptor activation 
on leukocytes via chemokines results in an increased expression of adhesion molecules of their cell surfaces; (iii) arrest: interactions between adhesion molecules on the brain endothelial cells and adhesion molecules on leukocytes; (iv) crawling: preferred site for transmigration is sought out by the leukocyte as it crawls along the brain endothelial cells; (v) transmigration: crossing of the leukocytes in between adjacent brain endothelial cells and into the brain parenchyma [100]. This multi-step process of leukocyte migration has been implicated in many neurological diseases like multiple sclerosis (MS), stroke, and life-threatening infections like sepsis [100102].

The infiltration of immune cells from the periphery into the brain is a central indication that other components of the brain endothelial cell (TJ proteins, actin cytoskeleton etc.) may be dysfunctional depending on the disease context. Loss of BBB integrity is well documented in neuropathologies like the MS and stroke [103]. Rochfort et al. and others have shown reduced expression of TJ proteins (occludin and claudin-5) and an upregulation of metalloproteinases (MMPs) in human brain microvascular endothelial cells after treatment with the serum of MS patients [104-107]. Furthermore, the reorganization of brain endothelium actin cytoskeleton via pro-inflammatory cytokines in MS patients contributes to the decreased expression of TJ proteins $[108,109]$. Likewise, oxidative stress on brain endothelial cells during the ischemic and reperfusion phases in stroke may also cause BBB dysfunction. Stroke oxygen-glucose deprivation in vitro and in vivo middle cerebral artery occlusion (MCAO) experiments conducted by Liu et al. showed that brain endothelial TJ proteins are decreased and redistributed alongside an associated increase MMP-2 [110].

Sepsis, particularly Gram-negative sepsis, has been shown to upregulate caveolin-1 in brain endothelial cells [111]. Increased caveolin-1 has recently been shown to increase the amount of peripheral immune infiltration into the brain [112]. The mechanisms by which this occurs are not completely understood, but new preclinical studies have shed light on some prevailing theories. Wu and colleagues found that caveolin-1 facilitates T-cell trafficking into the 
CNS via brain endothelial cell increased expression of intercellular adhesion molecule-1 (ICAM-

1) [113]. Caveolin-1 causes acid sphingomyelinase to interact with ICAM-1 increasing the binding affinity for peripheral immune cells [114]. Once activated, ICAM-1 facilitates peripheral immune cell diapedesis into the brain. This process occurs via Src phosphorylation in brain endothelial cells and a subsequent conformational change to ICAM-1, which directly induces transcellular migration [115]. During sepsis, this entire process is enhanced. After entering the brain, T-cells are recruited towards damaged glia via cytokine release. Recent evidence suggests that IL-17A aids this migration process [116]. Additionally, T-cells are helped by astrocytes to re-cross the leaky BBB and carry information about the status of the brain to the rest of the body [117]. It is postulated that peripheral immune cells also release cytokines that maintain the leakiness of the BBB as they exit the brain. When and how long this cross-talk between microglia and peripheral immune cells persist remains to be elucidated. The feedback loop, however, has been implemented in non-autonomous neuronal death [118].

Future studies are warranted to further characterize the brain/immune communication network and, in particular, where the peripheral immune cells ultimately reside after exiting the brain. Collectively, these mechanisms represent the complicated and multi-factorial mechanisms of sepsis at the level of the BBB. More importantly these studies emphasize a role for BMECs as a therapeutic target to treat many neurological conditions. An integrated overview of the brain and peripheral mechanisms found in sepsis is shown in Figure 4.

\section{IV: A Putative Role Tissue-nonspecific Alkaline Phosphatase (TNAP) in BMECs}

The identification of unexplored membrane proteins may be key to better understanding the specific barrier functions of the BBB in disease states such as sepsis. In turn, this knowledge may provide novel therapeutic targets for intervention. One potential therapeutic target localized primarily to the surface of brain endothelial cells is the non-specific isoform of alkaline phosphatase (AP), also known as TNAP. Tissue-nonspecific alkaline phosphatase 
(TNAP) is one of many proteins localized to BMECs, and is highly abundant in human and rodent cerebral microvessels [119]. There are four alkaline phosphatase (AP) isoenzymes and they include: TNAP, germ cell alkaline phosphatase (GCAP), intestinal alkaline phosphatase (IAP), and placental alkaline phosphatase [120]. TNAP is expressed in bone, liver, intestine, kidney, and brain while the other three AP isoenzymes are expressed in the tissues which they are aptly named after [121].

TNAP is bound to the plasma membranes of cells via a glycosylphosphatidylinositol (GPI) moiety, and functions as an ectoenzyme [122-124]. Known substrates for TNAP under healthy conditions include: inorganic pyrophosphates (PPi), vitamin B6, adenosine triphosphate (ATP), adenosine diphosphate (ADP), adenosine monophosphate (AMP), and phosphoethanolamine (PEA) $[125,126]$. A role for TNAP in bone is well documented from earlier studies showing that defective TNAP activity in bone led to hypophosphatasia in humans and rodents [127-129]. The observed defect in TNAP function demonstrates a role for TNAP in skeletal mineralization, and resulted from an accumulation of PPi and osteopontin (OPN) which blocks hydroxyapatite crystal formation - a key inorganic constituent required for bone formation $[130,131]$. Furthermore, patients who demonstrated clinical hypophosphatasia also presented with seizures [132]. Later studies, revealed that the seizures resulted from failure to transport vitamin B6 across the BBB. TNAP's inability to hydrolyze vitamin B6 in the periphery decreased the availability of pyridoxal, a hydrolyzed form of vitamin B6 which is able to cross the BBB but not vitamin B6; thus, this creates a deficiency of vitamin B6 in the brain which is important for synthesis of gamma aminobutyric acid (GABA), an inhibitory neurotransmitter [133, 134]. Despite the presumed role of TNAP in peripheral and CNS health from these elegant studies, it's role in cerebral microvessels remained vastly unexplored.

There are two different TNAP isoforms, so-called bone and liver TNAP isoforms. The bone-type TNAP transcript is expressed in the BMECs of human and mouse [135]. During development, mouse TNAP activity in cerebral microvessels is delayed and not seen until post- 
natal day 10 compared to rat and primate TNAP activity which is seen as early as embryonic day 15 [135], and at 28 weeks of gestation in humans [136]. Also, TNAP enzymatic activity in the brain parenchyma compared to cerebral microvessels differs from one species to another. For example, TNAP activity is weaker in the brain parenchyma compared to cerebral microvessels in humans, monkeys, rodents, guinea pigs, and cats [137-140]; however, in rabbits, frogs, and chickens, TNAP activity is stronger in the brain parenchyma but weaker in cerebral microvessels [137, 141]. Furthermore, differences between TNAP activity expression in peripheral vessels compared to cerebral microvessels have been elucidated. A comparative study assessing the expression of TNAP in BMECs compared to peripheral endothelial cells from the aorta showed that BMECs express a significant amount of TNAP activity compared to peripheral endothelial cells [142]. In addition, Vorbrodt et al. showed that TNAP in the endothelial cells of the liver sinusoids was absent, however, skeletal endothelial cells revealed a strong TNAP activity that was discontinuous or irregularly scattered across the plasma membrane compared to brain endothelial cells which showed a continuous and uniform layer of strong TNAP expression [143]. Such species-to-species or cell developmental differences need to be taken into account when elucidating the function of BMEC TNAP in animal models prior to translating these findings to human BMEC TNAP function. Despite the high expression of TNAP on cerebral microvessels, the role of TNAP in the brain microvascular endothelium remained unclear. This dissertation aims to demonstrate a role for TNAP in maintaining barrier integrity in health and in sepsis.

\section{Models and Tools to Study TNAP: Advantages and Disadvantages}

One of the many challenges to elucidating the role of TNAP in health and disease has been the creation of appropriate pharmacological and genetic tools. Although TNAP is present in all mammals and most tissues, its gene ( $A L P L$ in humans, Alpl or Akp2 in mice) transcription varies slightly [135]. In the first of its 12 exons, the 5'-untranslated region (UTR) contains either 
exon $1 \mathrm{~A}$ or $1 \mathrm{~B}$ by alternative transcription initiation [144]. Exon $1 \mathrm{~A}$ is preferentially driven in osteoblasts, while exon 1B is more often initiated by the liver and kidney [145-147]. Using marmosets, brain TNAP has been shown to use the same promotor as bone, however exon 1B is preferentially transcribed by mouse neurons [135]. TNAP activity is also important during mouse embryo development; it is the predominant AP expressed in 7 to 14 day-old embryos and primordial germ cells [148]. As of January 2021, there are 411 mutations reported in the ALPL gene mutations database (http://alplmutationdatabase.hypophosphatasie.com/), the majority of which are pathogenic. Dramatic depletion of TNAP activity, clinically referred to as hypophosphatasia (HPP), results in neurological symptoms, including epilepsy [130, 133, 149]. Conversely, abnormally high levels of TNAP activity can also result in seizures and neurological dysfunction [150-152]. This presents an interesting dynamic where TNAP activity levels must be sustained within a certain range in order to maintain homeostasis.

\section{A. TNAP mouse models}

A recent review describes several models for HPP where the TNAP gene is either genetically or chemically depleted [153]. Readers are referred to this review which covers indepth genetically modified mice for evaluation of TNAP function [154]. TNAP knock-out $\left(A / p /^{H^{-}}\right)$ mice life expectancy is short, averaging $3-5$ weeks $[133,155]$; therefore, they cannot be used for adult studies of HPP or to elucidate the function of TNAP in brain microvessels. Some researchers opt to use adult heterozygous transgenic mice $\left(A / \mathrm{pl}^{+/-}\right)$to explore the consequences of reduced TNAP activity [156-158]. Injection of a recombinant mineral-targeted TNAP lentivirus into neonatal mice has been shown to increase AP levels up to 60 days and reduces craniosynostosis $[159,160]$. Due to the wide expression of TNAP in various cell types, TNAPexpressing cells can also be targeted using the Cre-Lox system. For example, using mice with tamoxifen-inducible inactivation of transforming growth factor $ß$ (TGF-ß) in TNAP-expressing 
cells (Tnap $\left.{ }^{\text {cre }} ; T g f ß r 2^{f / / f l}\right)$, researchers have shown that TNAP mitigates TGF-ß-dependent cardiac and skeletal muscle fibrosis through inactivation of SMAD2/3 transcription factors [161].

Conditional TNAP overexpression has been achieved using an $H_{p r}{ }^{\mathrm{ALPL}}$ knock-in mouse, which is described in [162]. Briefly, this model contains a floxed "stop cassette" and human ALPL cDNA is inserted into the hypoxanthine phosphoribosyltransferase (Hprt) locus on the $\mathrm{X}$ chromosome. Cross-breeding of the $\mathrm{Hprt}^{\mathrm{ALPL}}$ with Cre-expressing mice results in deletion of the stop cassette and constitutive ALPL expression within the target cell type. Because of the nature of this X-linked system, both homozygous (Hprt $\left.{ }^{\mathrm{ALPL} / \mathrm{ALPL}}\right)$ and heterozygous $\left(\mathrm{Hprt}^{\mathrm{ALPL} / \mathrm{s}}\right)$ females are considered to be over-expressors, however Hprt ${ }^{\mathrm{ALPL} /-}$ females typically show a milder phenotype. Female Tagln-Cre ${ }^{+/-} ; \operatorname{Hprt} A L P L^{/-}$mice showed a much milder medial vascular calcification (MVC) phenotype compared to male hemizygous Tagln-Cre ${ }^{+/-} ; H_{p r t A L P L}{ }^{\top}$ mice, however the effects in female Tagln-Cre ${ }^{+/-} ; \operatorname{HprtALPL}{ }^{\text {IALPL }}$ mice were not described [162]. Creation of $A / p f^{f / / f l}$ mice was first described here [163]; a floxed Alpl allele allows for conditional knockout of TNAP when crossed with Cre recombinase transgenic mice. Breeding of the appropriate combinations of Cre and $A / p f^{f / / f l}$ animals after three generations will result in $50 \%$ of the offspring as TNAP-cKO mice and $50 \%$ as $A / p f^{f / f f l}$ littermate controls.

\section{B. Pharmaceutical tools to study TNAP}

There are several commercially available TNAP inhibitors currently on the market. Lhomoarginine (hArg) and levamisole are two of the oldest inhibitors, however these are not very selective and offer weak binding to TNAP and are known to cause neurological side effects [164, 165]. In 2019, researchers showed that hArg had no effect on TNAP levels in TNAPoverexpressing mice, however, it did offer some protection from myocardial remodeling through mechanisms unrelated to TNAP inhibition [166]. Although levamisole has been used in several studies to help identify the role that TNAP plays in health and disease $[119,167,168]$, it is a reversible inhibitor, so cells treated with this drug rapidly regain TNAP activity [169]. Due to their 
lack of specificity and negative effects of pan-AP inhibitors such as levamisole, Dr. Jose Luis Millan and collaborators sought to develop a better inhibitor for TNAP. Using high-throughput screening they identified several small molecule Aryl Sulfonamides as potent and selective TNAP inhibitors [170]. So far, one of these inhibitors is commercially available for purchase from Millipore Sigma, 2,5-Dimethoxy-N-(quinoline-3-yl) benzenesulfonamide or MLS-0038949, primarily for cell structure applications. Further modifications of the previous TNAP inhibitor has resulted in the development of a second inhibitor more suitable for in vivo applications, SBI-425 [171]. SBI-425 has been used in several studies related to vascular calcification [162, 172, 173] and by our own lab to identify its applicability for studying TNAP's role in the brain; however, we demonstrated that the SBI-425 inhibitor is unable to cross the healthy BBB [174].

\section{Project Objectives and Aims}

BMECs are a principal component of the BBB that provide the structural framework needed for the stringent transportation of molecules into the brain, and maintenance of precise cerebral homeostasis. BMEC dysfunction permits the trafficking of neurotoxins, bacteria, and inflammatory cells from systemic circulation into the brain, which ultimately exacerbates BBB dysfunction and neuroinflammation. Recent studies have shown that BBB dysfunction is a key determinant of cognitive decline [79, 175-177]. Yet, therapies that mitigate BBB dysfunction and improve cognitive outcomes in neurogenerative and inflammatory disease conditions are limited because of the critical knowledge gap that exists in understanding the molecular and cellular mechanisms underpinning BMEC physiology and function as it relates to the maintenance of

\section{$\underline{B B B \text { integrity. }}$}

A strategy for bridging this critical knowledge gap requires an examination of protein and enzymes localized to BMECs, and evaluating their role in preserving BBB integrity. TNAP has been historically used as a brain endothelial histological marker due to its abundant expression on BMECs. Yet, the role of TNAP in BMECs remains unclear. The objective of this dissertation 
is to elucidate the role played by BMEC TNAP in preserving BBB integrity in normal physiology and in sepsis. Taken together, we will test the central hypothesis that BMEC TNAP is critical for maintenance of BBB integrity in normal physiology and in sepsis through molecular mechanisms that preserve BMEC structure and function. We will test our central hypothesis via the following 3 specific aims:

Aim 1: Establish a role for brain microvascular TNAP in early and late sepsis (covered in Chapter 2). Decades of studies have shown that cerebral microvessels are abundantly rich in the AP isoenzyme known as TNAP $[124,178,179]$. Yet, TNAP's role on the cerebral microvessels remains to be elucidated [119]. The objective of this aim is to demonstrate a role for TNAP in sepsis, and provide insights to how loss of brain microvascular TNAP couples sepsis-associated BBB dysfunction and neuroinflammation.

\footnotetext{
Aim 2: Elucidate the molecular mechanisms through which BMEC TNAP maintains barrier integrity (covered in Chapter 3). Preliminary evidence from earlier TNAP studies have demonstrated that TNAP is able to hydrolyze nucleotides (ATP, AMP, ADP), inorganic pyrophosphates (PPi), vitamin B6, and PEA [125, 126]. However, these targets are not specific to brain endothelial cells and represent upstream pathways through which TNAP operates. The objective of this aim is to elucidate downstream molecular targets of BMEC TNAP that could be therapeutically advantageous towards the treatment of sepsis and prevention of long-term cognitive impairment.
}

\footnotetext{
Aim 3: Determine the impact of BMEC TNAP conditional loss on BBB integrity in health and sepsis (covered in Chapter 4). Other cell types that comprise the BBB such as astrocyte end-feet processes and pericytes have been shown to express TNAP activity [180-182]. The
} 
objective of this aim is to examine whether the conditional loss of BMEC TNAP in vivo is capable of inducing BBB dysfunction in health and sepsis.

The expected outcome of this work will produce a comprehensive understanding of the role played by BMEC TNAP activity in preserving BBB integrity in normal physiology and in the setting of sepsis-induced neurological impairment. The results will have a positive impact because they lay the groundwork needed to stimulate the discovery of a potential therapeutic target whose activity can be manipulated during injury to mitigate long-term neurological dysfunction in sepsis, or alternatively, as a tool to increase the permeability of the BBB for temporary delivery of drugs to the brain.

\section{VIl. Conclusion}

The heterogeneous presentation and causes of sepsis are profoundly linked to its variable clinical outcomes. Chronic neurological impairment is an increasingly common yet poorly understood clinical outcome. Understanding the mechanistic determinants of BBB integrity during sepsis is critically important for sepsis diagnosis and implementation of treatment options to ensure a positive prognosis. Importantly, long-term prognosis in sepsis survivors is linked to both transient and permanent alterations in BBB permeability and function. Thus, targeting the BBB should be incorporated as part of a short- and long-term therapeutic strategy in all sepsis patients. The development of therapies that inhibit BBB dysfunction and stimulate normal BBB function will limit mortality, suppress neuroinflammation, and improve neurological outcomes in sepsis survivors. Taken together, the identification of cellular and molecular mechanisms that preserve BBB function in the face of sepsis will provide valuable therapeutic targets to treat numerous inflammatory disorders that target both the brain and the periphery-ranging from $A D$ and stroke to diabetes and cardiovascular disease. 


\section{Figures}

\section{Figure 1}

A.

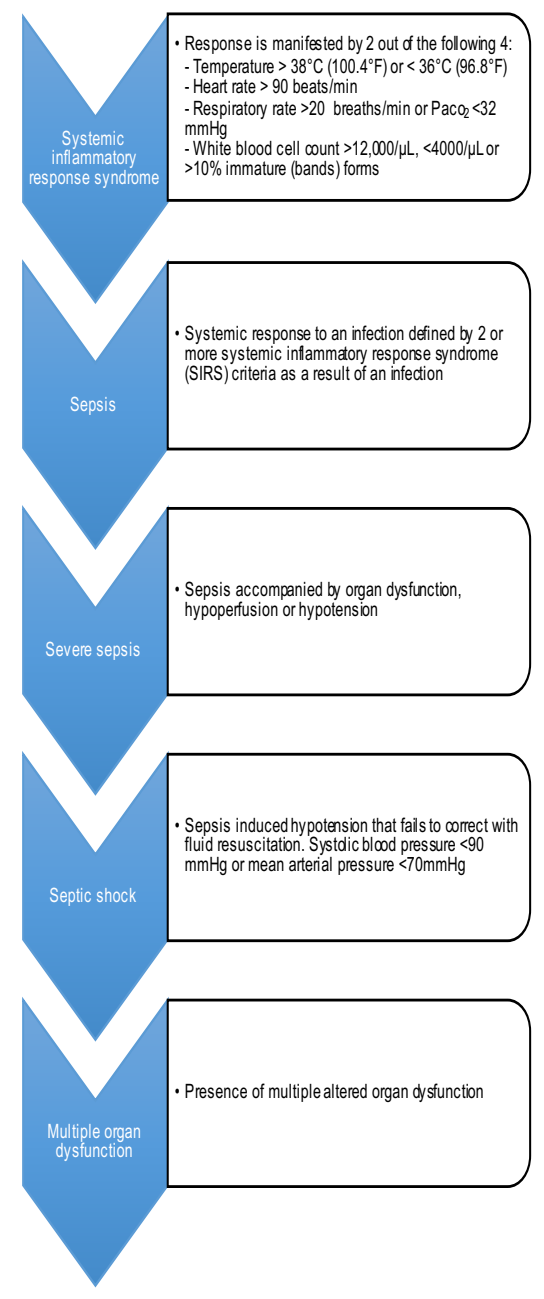

B.
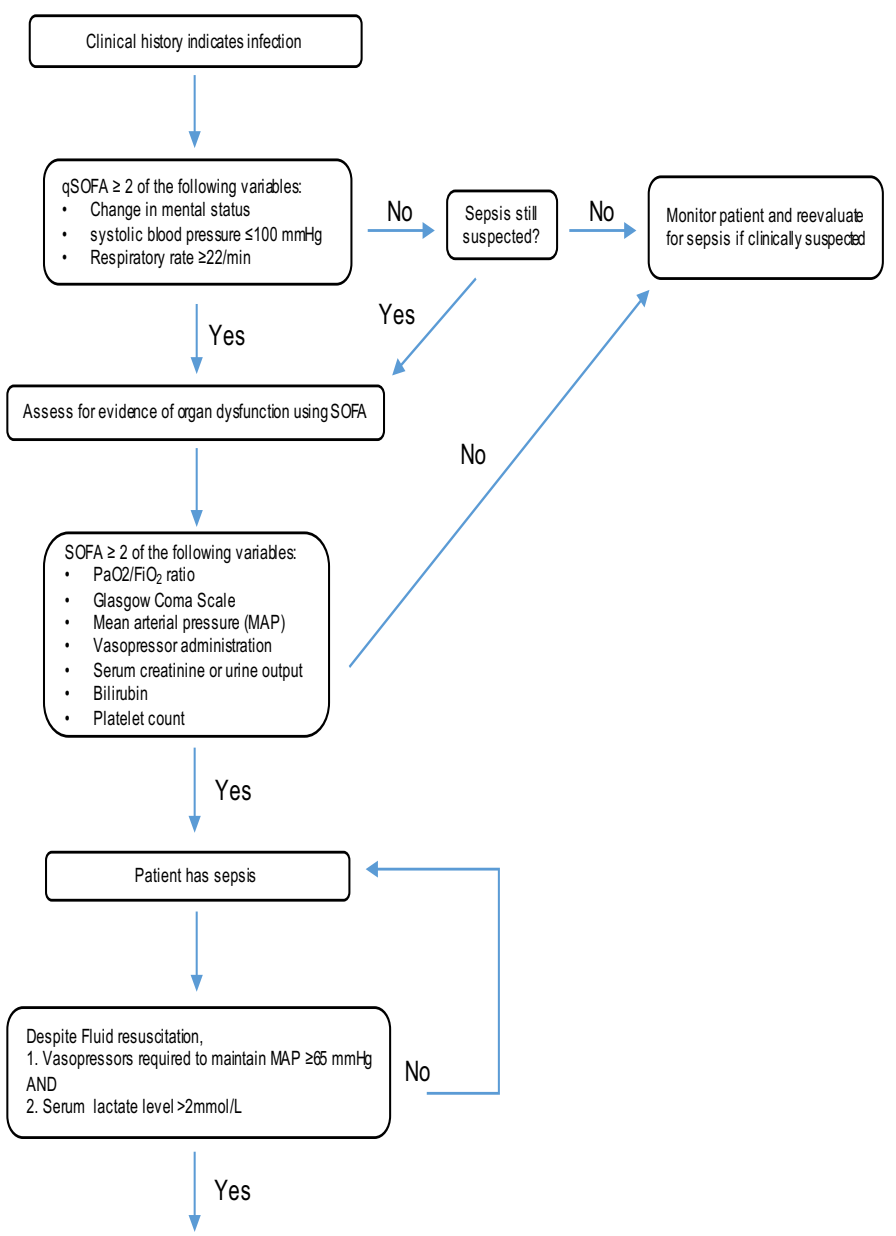

Patient has septic shock 
Figure 2

Sham $\quad$ CLP $-22 g \quad C L P-20 g$

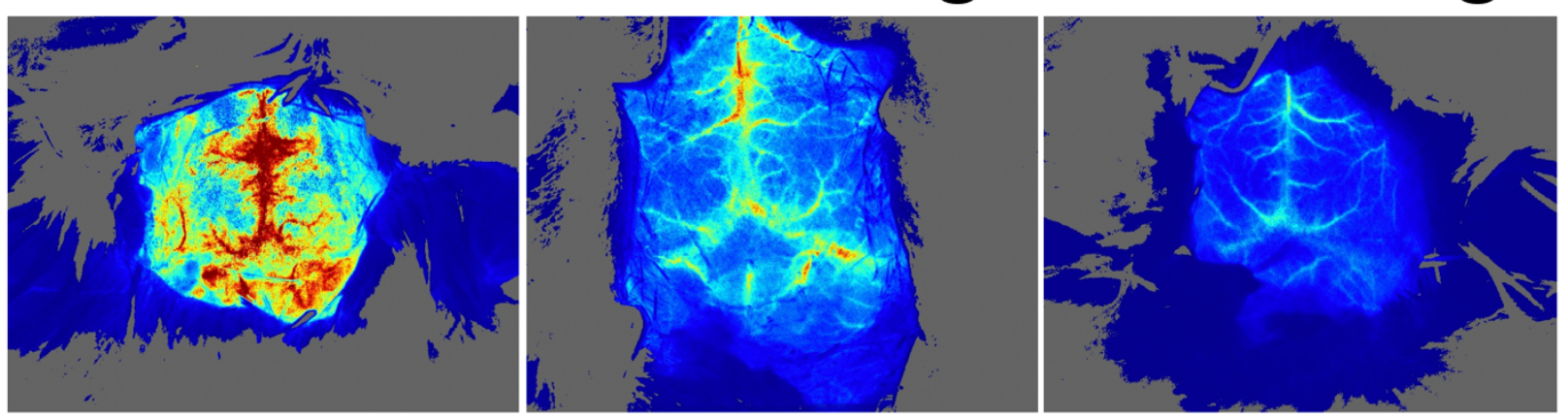


Figure 3

\section{CNS}

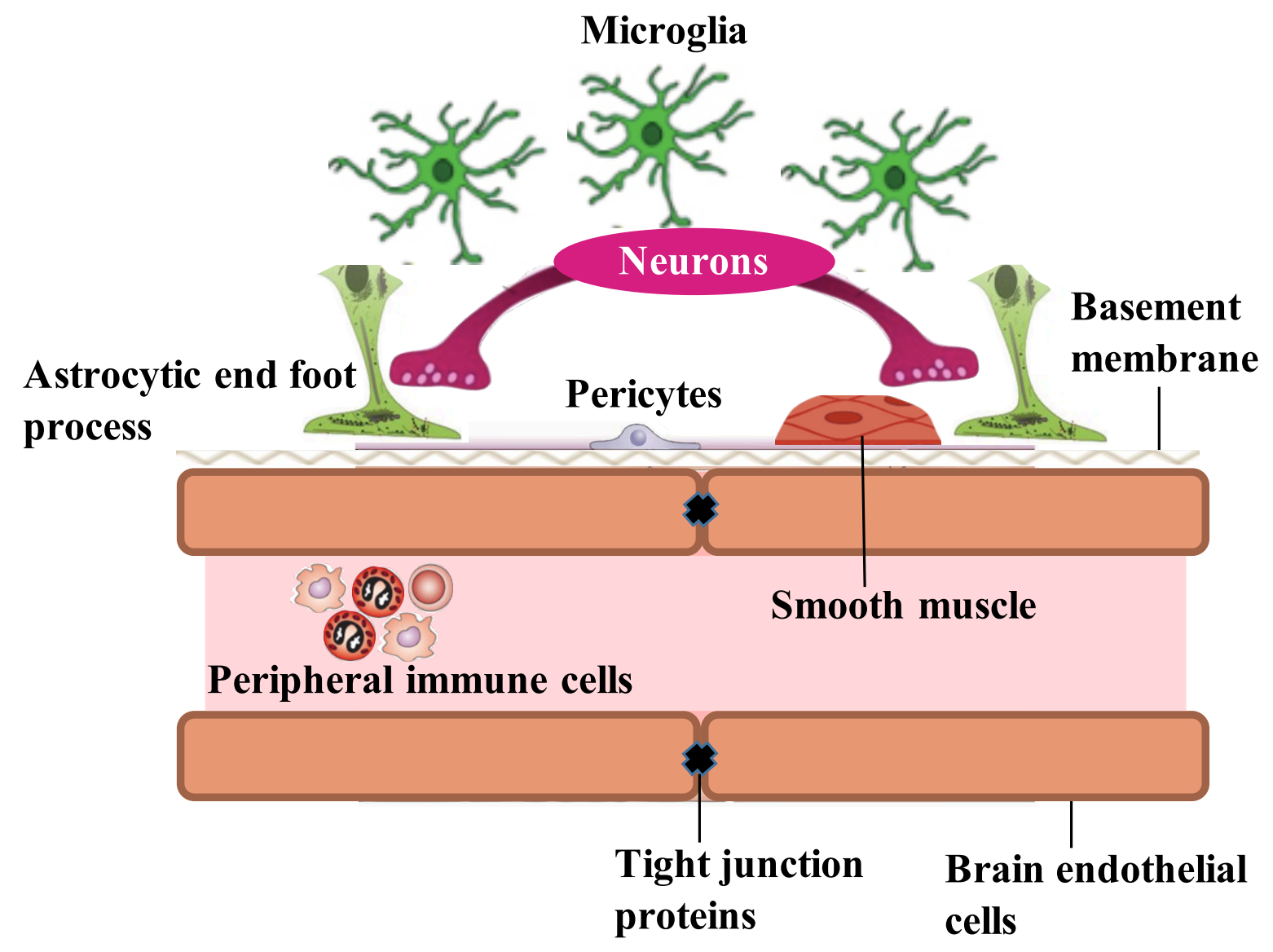




\section{Figure 4}

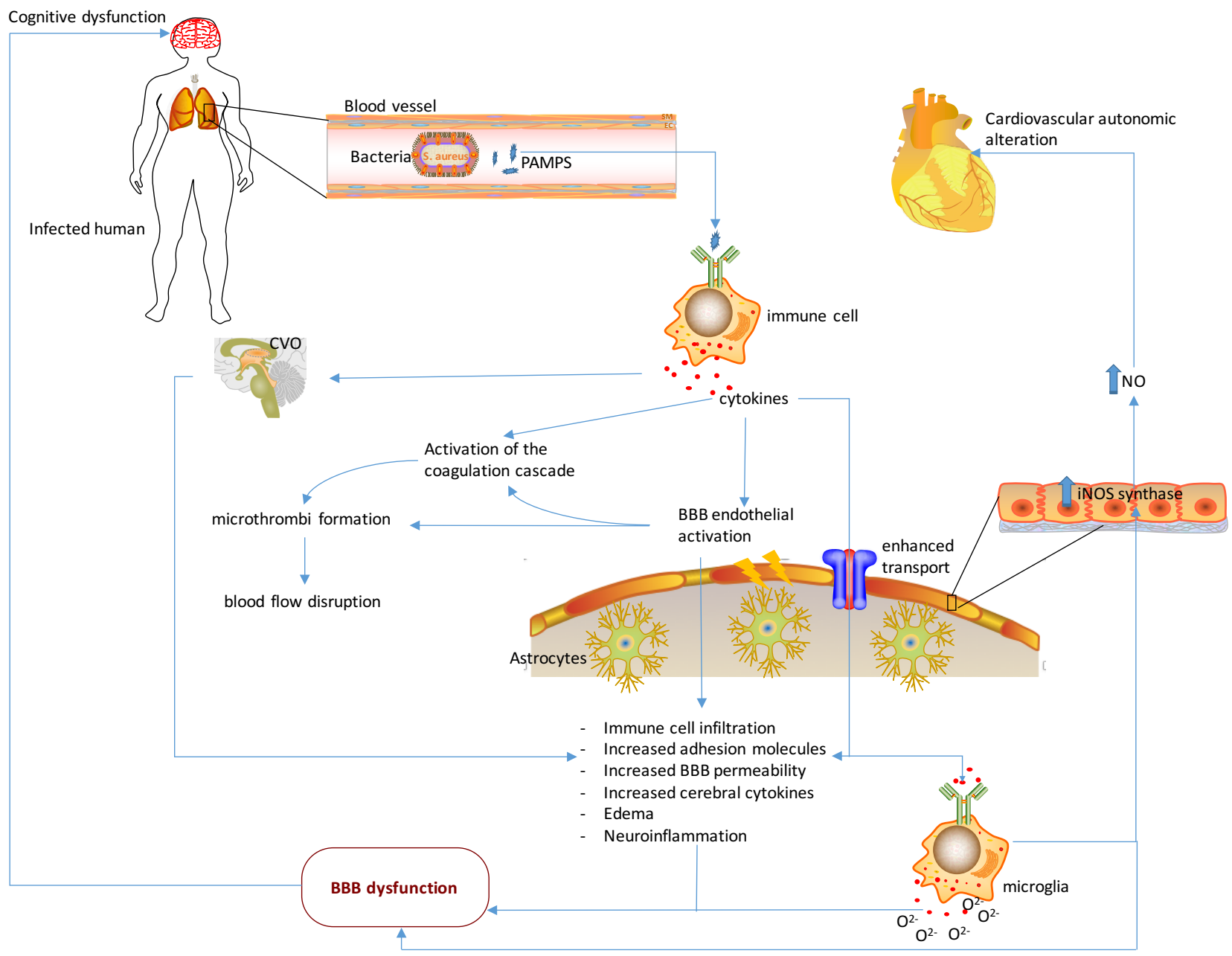

Figure Legends

Figure 1: Comparison of past and present guidelines for diagnosis of sepsis and septic shock. (A) Past guidelines stressed that the diagnosis and progression of sepsis from a 
systemic inflammatory response to multiple organ dysfunction was sequential rather than multifactorial. (B) The current guidelines for defining sepsis and septic shock stress that multiple linked considerations are necessary for an accurate diagnosis. Both past and present guidelines involve a clinical screening tool for patients likely to have sepsis that includes a clinical characterization of the severity of the disease. Clinicians have traditionally used a sequential organ failure assessment (SOFA) system to categorize the severity of organ dysfunction in sepsis, which associates a higher SOFA score with increased mortality [4, 183]. New sepsis guidelines employ a quick SOFA (qSOFA) score, which is a modified version of the SOFA score that includes altered mental status, a systolic blood pressure $\leq 100 \mathrm{mg} \mathrm{Hg}$, and a respiratory rate $\geq 22 / \mathrm{min}$. Patients with a qSOFA $\geq 2$ have an overall in-hospital mortality risk greater than $10 \%[4,184]$. If warranted, further clinical analysis can be completed using other SOFA criteria [4]. Figure is adapted from Singer et al. [4].

Figure 2: Relative cerebrovascular blood flow post-sepsis. Male C57BL/6J mice were subjected to two different CLP severity models of sepsis (22 gauge: severe sepsis; 20 gauge: septic shock). Relative blood flow was measured $12 \mathrm{~h}$ post-sepsis using a laser speckle instrument. As expected, the septic shock mice demonstrated reduced cerebrovascular blood flow compared to the severe sepsis and sham mice. This diagram illustrates the impact of sepsis on the cerebrovasculature.

Figure 3 Key cellular components of the blood-brain barrier. The schematic illustrates the relationships between cells that comprise the BBB. Neurons and microglia are also included as part of the neurovascular unit, a conceptual framework that describes the interactions with the $\mathrm{BBB}$, resident brain cells, and peripheral immune cells.

Figure 4: Complex neuroinflammatory processes disrupt the blood brain barrier (BBB) and lead to cognitive impairment in sepsis. Sepsis begins with systemic infection that evokes an exaggerated host immune response from the recognition of pathogen-associated molecular patterns (PAMPs) [90]. This exaggerated immune response involves a cascade of events, including disrupted blood flow, immune cell infiltration, and increased BBB permeability, which may ultimately result in cognitive dysfunction [95-97]. 
Tables

Table 1: MRI imaging studies in patients with sepsis

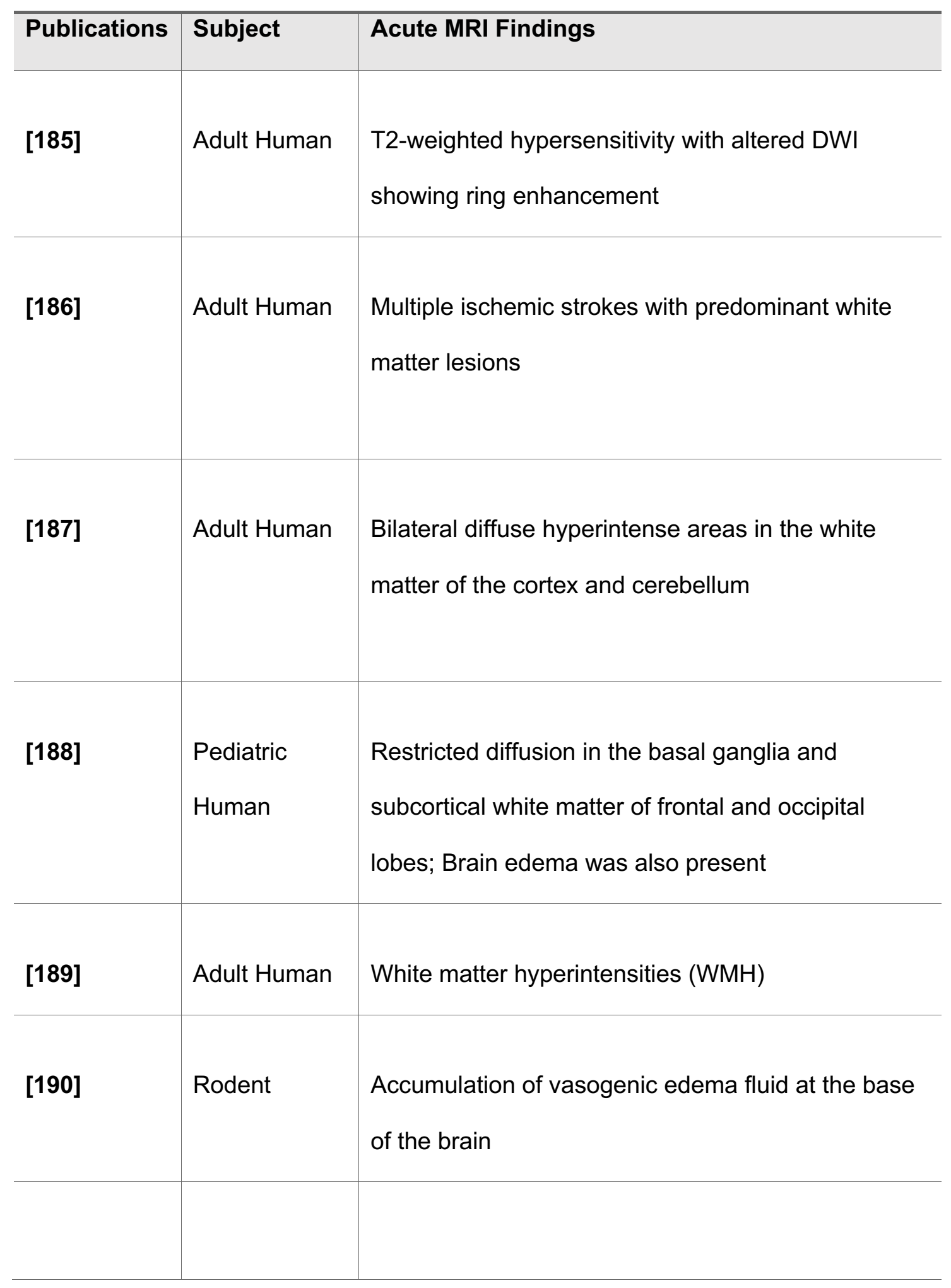




\begin{tabular}{l|l|l}
\hline [191] & Adult Human & $\begin{array}{l}\text { Atrophy, WMH, edema, and localized bilateral } \\
\text { hemorrhage in the cortex and subcortical structures }\end{array}$ \\
\hline [57] & Adult Human & Leukoencephalopathy and ischemic stroke \\
\hline [192] & Adult Human & Acute abnormal hypointensity of the white matter \\
& and edema; Subacute WMH \\
\hline [58] & Rodent & Increased MRI intensities in the cortex, perirhinal \\
& cortex, and hippocampus; Increased gadolinium \\
& infiltration into various brain regions
\end{tabular}




\section{References}

1. Martin, A.B., et al., National Health Spending In 2014: Faster Growth Driven By Coverage Expansion And Prescription Drug Spending. Health Aff (Millwood), 2016. 35(1): p. 150-60.

2. Fingar, K. and R. Washington, Trends in Hospital Readmissions for Four High-Volume Conditions, 2009-2013: Statistical Brief \#196, in Healthcare Cost and Utilization Project (HCUP) Statistical Briefs. 2006: Rockville (MD).

3. Stearns-Kurosawa, D.J., et al., The pathogenesis of sepsis. Annu Rev Pathol, 2011. 6: p. 19-48.

4. $\quad$ Singer, M., et al., The Third International Consensus Definitions for Sepsis and Septic Shock (Sepsis-3). JAMA, 2016. 315(8): p. 801-10.

5. Martin, G.S., Sepsis, severe sepsis and septic shock: changes in incidence, pathogens and outcomes. Expert Rev Anti Infect Ther, 2012. 10(6): p. 701-6.

6. Mayr, F.B., S. Yende, and D.C. Angus, Epidemiology of severe sepsis. Virulence, 2014. 5(1): p. 4-11.

7. Mahapatra, S. and A.C. Heffner, Shock, Septic (Sepsis), in StatPearls. 2018: Treasure Island (FL).

8. Laszlo, I., et al., Sepsis: From Pathophysiology to Individualized Patient Care. J Immunol Res, 2015. 2015: p. 510436.

9. Monai, H., et al., Adrenergic receptor antagonism induces neuroprotection and facilitates recovery from acute ischemic stroke. Proc Natl Acad Sci U S A, 2019. 116(22): p. 11010-11019.

10. Macdonald, S.P., et al., Review article: Sepsis in the emergency department - Part 1: Definitions and outcomes. Emerg Med Australas, 2017. 29(6): p. 619-625.

11. Morr, M., et al., Sepsis recognition in the emergency department - impact on quality of care and outcome? BMC Emerg Med, 2017. 17(1): p. 11.

12. Fleischmann, C., et al., Hospital Incidence and Mortality Rates of Sepsis. Dtsch Arztebl Int, 2016. 113(10): p. 159-66.

13. Iskander, K.N., et al., Sepsis: multiple abnormalities, heterogeneous responses, and evolving understanding. Physiol Rev, 2013. 93(3): p. 1247-88.

14. Martin, G.S., et al., The epidemiology of sepsis in the United States from 1979 through 2000. N Engl J Med, 2003. 348(16): p. 1546-54.

15. Esper, A.M., et al., The role of infection and comorbidity: Factors that influence disparities in sepsis. Crit Care Med, 2006. 34(10): p. 2576-82.

16. Kingsley, S.M. and B.V. Bhat, Differential Paradigms in Animal Models of Sepsis. Curr Infect Dis Rep, 2016. 18(9): p. 26.

17. Parker, S.J. and P.E. Watkins, Experimental models of gram-negative sepsis. Br J Surg, 2001. 88(1): p. 22-30.

18. Dantzer, R., Cytokine-induced sickness behavior: where do we stand? Brain Behav Immun, 2001. 15(1): p. 7-24.

19. Zantl, N., et al., Essential role of gamma interferon in survival of colon ascendens stent peritonitis, a novel murine model of abdominal sepsis. Infect Immun, 1998. 66(5): p. 2300-9.

20. Traeger, T., et al., Colon ascendens stent peritonitis (CASP)--a standardized model for polymicrobial abdominal sepsis. J Vis Exp, 2010(46).

21. Kerschen, E.J., et al., Endotoxemia and sepsis mortality reduction by non-anticoagulant activated protein C. J Exp Med, 2007. 204(10): p. 2439-48.

22. Michie, H.R., et al., Detection of circulating tumor necrosis factor after endotoxin administration. N Engl J Med, 1988. 318(23): p. 1481-6. 
23. Wyler, F., J.M. Neutze, and A.M. Rudolph, Effects of endotoxin on distribution of cardiac output in unanesthetized rabbits. Am J Physiol, 1970. 219(1): p. 246-51.

24. Fink, M.P. and S.O. Heard, Laboratory models of sepsis and septic shock. J Surg Res, 1990. 49(2): p. 186-96.

25. Fink, M.P., et al., Systemic and regional hemodynamic changes after intraperitoneal endotoxin in rabbits: development of a new model of the clinical syndrome of hyperdynamic sepsis. Circ Shock, 1987. 22(1): p. 73-81.

26. Buras, J.A., B. Holzmann, and M. Sitkovsky, Animal models of sepsis: setting the stage. Nat Rev Drug Discov, 2005. 4(10): p. 854-65.

27. Deutschman, C.S. and K.J. Tracey, Sepsis: current dogma and new perspectives. Immunity, 2014. 40(4): p. 463-75.

28. Barichello, T., et al., Long-Term Cognitive Outcomes After Sepsis: a Translational Systematic Review. Mol Neurobiol, 2018.

29. Polat, G., et al., Sepsis and Septic Shock: Current Treatment Strategies and New Approaches. Eurasian J Med, 2017. 49(1): p. 53-58.

30. Hotchkiss, R.S., et al., Sepsis and septic shock. Nat Rev Dis Primers, 2016. 2: p. 16045.

31. Kirsten, T.B., et al., Zinc prevents sickness behavior induced by lipopolysaccharides after a stress challenge in rats. PLoS One, 2015. 10(3): p. e0120263.

32. Kessler, W., et al., The role of the vagus nerve: modulation of the inflammatory reaction in murine polymicrobial sepsis. Mediators Inflamm, 2012. 2012: p. 467620.

33. Mitsui, T., et al., Truncal vagotomy temporarily decreases the pro- and anti-inflammatory cytokine levels in the small intestine. Surg Today, 2014. 44(6): p. 1123-7.

34. Tracey, K.J., The inflammatory reflex. Nature, 2002. 420(6917): p. 853-9.

35. Borovikova, L.V., et al., Vagus nerve stimulation attenuates the systemic inflammatory response to endotoxin. Nature, 2000. 405(6785): p. 458-62.

36. Li, N., et al., [Protective effects of vagus nerve stimulation on rats with sepsis-associated encephalopathy]. Zhonghua Wei Zhong Bing Ji Jiu Yi Xue, 2015. 27(6): p. 509-13.

37. Wilcock, D.M., et al., Progression of amyloid pathology to Alzheimer's disease pathology in an amyloid precursor protein transgenic mouse model by removal of nitric oxide synthase 2. J Neurosci, 2008. 28(7): p. 1537-45.

38. Mazeraud, A., et al., Neuroanatomy and Physiology of Brain Dysfunction in Sepsis. Clin Chest Med, 2016. 37(2): p. 333-45.

39. Reyes, E.P., et al., LPS-induced c-Fos activation in NTS neurons and plasmatic cortisol increases in septic rats are suppressed by bilateral carotid chemodenervation. Adv Exp Med Biol, 2012. 758: p. 185-90.

40. Annane, D. and T. Sharshar, Cognitive decline after sepsis. Lancet Respir Med, 2015. 3(1): p. 61-9.

41. Pun, B.T. and J. Dunn, The sedation of critically ill adults: Part 1: Assessment. The first in a two-part series focuses on assessing sedated patients in the ICU. Am J Nurs, 2007. 107(7): p. 40-8; quiz 49.

42. Pytel, P. and J.J. Alexander, Pathogenesis of septic encephalopathy. Curr Opin Neurol, 2009. 22(3): p. 283-7.

43. vom Steeg, L.G. and S.L. Klein, SeXX Matters in Infectious Disease Pathogenesis. PLoS Pathog, 2016. 12(2): p. e1005374.

44. Hosokawa, K., et al., Clinical neurophysiological assessment of sepsis-associated brain dysfunction: a systematic review. Crit Care, 2014. 18(6): p. 674.

45. Chaudhry, N. and A.K. Duggal, Sepsis Associated Encephalopathy. Adv Med, 2014. 2014: p. 762320.

46. Gilmore, E.J., et al., Acute brain failure in severe sepsis: a prospective study in the medical intensive care unit utilizing continuous EEG monitoring. Intensive Care Med, 2015. 41(4): p. 686-94. 
47. Semmler, A., et al., Persistent cognitive impairment, hippocampal atrophy and EEG changes in sepsis survivors. J Neurol Neurosurg Psychiatry, 2013. 84(1): p. 62-9.

48. Piazza, O., et al., Elevated S100B levels do not correlate with the severity of encephalopathy during sepsis. Br J Anaesth, 2007. 99(4): p. 518-21.

49. Sinclair, J.F., et al., Cerebral blood flow is reduced in patients with sepsis syndrome. Crit Care Med, 1990. 18(6): p. 684.

50. Brassard, P., et al., Endotoxemia reduces cerebral perfusion but enhances dynamic cerebrovascular autoregulation at reduced arterial carbon dioxide tension. Crit Care Med, 2012. 40(6): p. 1873-8.

51. Semmler, A., et al., Sepsis causes neuroinflammation and concomitant decrease of cerebral metabolism. J Neuroinflammation, 2008. 5: p. 38.

52. Taccone, F.S., et al., Cerebral microcirculation is impaired during sepsis: an experimental study. Crit Care, 2010. 14(4): p. R140.

53. Sharshar, T., et al., The neuropathology of septic shock. Brain Pathol, 2004. 14(1): p. 21-33.

54. Janz, D.R., et al., Brain autopsy findings in intensive care unit patients previously suffering from delirium: a pilot study. J Crit Care, 2010. 25(3): p. 538 e7-12.

55. Crippa, I.A., et al., Impaired cerebral autoregulation is associated with brain dysfunction in patients with sepsis. Crit Care, 2018. 22(1): p. 327.

56. Goodson, C.M., et al., Cerebral Blood Flow Autoregulation in Sepsis for the Intensivist: Why Its Monitoring May Be the Future of Individualized Care. J Intensive Care Med, 2018. 33(2): p. 63-73.

57. Polito, A., et al., Pattern of brain injury in the acute setting of human septic shock. Crit Care, 2013. 17(5): p. R204.

58. Towner, R.A., et al., Assessing long-term neuroinflammatory responses to encephalopathy using MRI approaches in a rat endotoxemia model. Geroscience, 2018. 40(1): p. 49-60.

59. Myburgh, J.A., et al., Hydroxyethyl starch or saline for fluid resuscitation in intensive care. N Engl J Med, 2012. 367(20): p. 1901-11.

60. Brunkhorst, F.M., et al., Intensive insulin therapy and pentastarch resuscitation in severe sepsis. N Engl J Med, 2008. 358(2): p. 125-39.

61. Perner, A., et al., Hydroxyethyl starch 130/0.42 versus Ringer's acetate in severe sepsis. N Engl J Med, 2012. 367(2): p. 124-34.

62. Avila, A.A., et al., The Use of Fluids in Sepsis. Cureus, 2016. 8(3): p. e528.

63. Semler, M.W. and T.W. Rice, Sepsis Resuscitation: Fluid Choice and Dose. Clin Chest Med, 2016. 37(2): p. 241-50.

64. Vincent, J.L. and L. Gottin, Type of fluid in severe sepsis and septic shock. Minerva Anestesiol, 2011. 77(12): p. 1190-6.

65. Stubbs, D.J., A.K. Yamamoto, and D.K. Menon, Imaging in sepsis-associated encephalopathy--insights and opportunities. Nat Rev Neurol, 2013. 9(10): p. 551-61.

66. Abe, S., et al., Sepsis associated encephalopathy in an infant with biliary atresia. Brain Dev, 2008. 30(8): p. 544-7.

67. Piazza, O., et al., Sepsis associated encephalopathy studied by MRI and cerebral spinal fluid S100B measurement. Neurochem Res, 2009. 34(7): p. 1289-92.

68. Bartynski, W.S., et al., Posterior reversible encephalopathy syndrome in infection, sepsis, and shock. AJNR Am J Neuroradiol, 2006. 27(10): p. 2179-90.

69. Bhaskaran, A., et al., Nephrogenic systemic fibrosis following acute kidney injury and exposure to gadolinium. Indian J Med Sci, 2010. 64(1): p. 33-6.

70. Schlaudecker, J.D. and C.R. Bernheisel, Gadolinium-associated nephrogenic systemic fibrosis. Am Fam Physician, 2009. 80(7): p. 711-4. 
71. Chien, C.C., et al., Risk of acute kidney injury after exposure to gadolinium-based contrast in patients with renal impairment. Ren Fail, 2011. 33(8): p. 758-64.

72. Qiao, H., et al., Sedation improves early outcome in severely septic Sprague Dawley rats. Crit Care, 2009. 13(4): p. R136.

73. Zamani, M.M., et al., Survival benefits of dexmedetomidine used for sedating septic patients in intensive care setting: A systematic review. J Crit Care, 2016. 32: p. 93-100.

74. Ma, D., et al., Dexmedetomidine produces its neuroprotective effect via the alpha 2Aadrenoceptor subtype. Eur J Pharmacol, 2004. 502(1-2): p. 87-97.

75. Maes, M., et al., The effects of noradrenaline and alpha-2 adrenoceptor agents on the production of monocytic products. Psychiatry Res, 2000. 96(3): p. 245-53.

76. Taniguchi, T., et al., Effects of dexmedetomidine on mortality rate and inflammatory responses to endotoxin-induced shock in rats. Crit Care Med, 2004. 32(6): p. 1322-6.

77. Pandharipande, P.P., et al., Long-term cognitive impairment after critical illness. N Engl J Med, 2013. 369(14): p. 1306-16.

78. Gunther, M.L., et al., The association between brain volumes, delirium duration, and cognitive outcomes in intensive care unit survivors: the VISIONS cohort magnetic resonance imaging study*. Crit Care Med, 2012. 40(7): p. 2022-32.

79. Iwashyna, T.J., et al., Long-term cognitive impairment and functional disability among survivors of severe sepsis. JAMA, 2010. 304(16): p. 1787-94.

80. Varatharaj, A. and I. Galea, The blood-brain barrier in systemic inflammation. Brain Behav Immun, 2017. 60: p. 1-12.

81. De Luca, C., et al., Neuro-Immune Hemostasis: Homeostasis and Diseases in the Central Nervous System. Front Cell Neurosci, 2018. 12: p. 459.

82. Abbott, N.J., et al., Structure and function of the blood-brain barrier. Neurobiol Dis, 2010. 37(1): p. 13-25.

83. Abbott, N.J., Blood-brain barrier structure and function and the challenges for CNS drug delivery. J Inherit Metab Dis, 2013. 36(3): p. 437-49.

84. Geldenhuys, W.J., et al., Molecular determinants of blood-brain barrier permeation. Ther Deliv, 2015. 6(8): p. 961-71.

85. Adkins, C.E., et al., P-glycoprotein mediated efflux limits substrate and drug uptake in a preclinical brain metastases of breast cancer model. Front Pharmacol, 2013. 4: p. 136.

86. Banks, W.A., From blood-brain barrier to blood-brain interface: new opportunities for CNS drug delivery. Nat Rev Drug Discov, 2016. 15(4): p. 275-92.

87. Zhao, Z., et al., Establishment and Dysfunction of the Blood-Brain Barrier. Cell, 2015. 163(5): p. 1064-1078.

88. Keaney, J. and M. Campbell, The dynamic blood-brain barrier. FEBS J, 2015. 282(21): p. 4067-79.

89. Ransohoff, R.M., et al., Neuroinflammation: Ways in Which the Immune System Affects the Brain. Neurotherapeutics, 2015. 12(4): p. 896-909.

90. Annane, D., E. Bellissant, and J.-M. Cavaillon, Septic shock. The Lancet, 2005. 365(9453): p. 63-78.

91. Erickson, M.A. and W.A. Banks, Neuroimmune Axes of the Blood-Brain Barriers and Blood-Brain Interfaces: Bases for Physiological Regulation, Disease States, and Pharmacological Interventions. Pharmacol Rev, 2018. 70(2): p. 278-314.

92. Akrout, N., T. Sharshar, and D. Annane, Mechanisms of brain signaling during sepsis. Curr Neuropharmacol, 2009. 7(4): p. 296-301.

93. Pan, W. and A.J. Kastin, TNFalpha transport across the blood-brain barrier is abolished in receptor knockout mice. Exp Neurol, 2002. 174(2): p. 193-200.

94. Nishijima, T., et al., Neuronal Activity Drives Localized Blood-Brain-Barrier Transport of Serum Insulin-like Growth Factor-I into the CNS. Neuron, 2010. 67(5): p. 834-846. 
95. Hughes, C.G., M.B. Patel, and P.P. Pandharipande, Pathophysiology of acute brain dysfunction: what's the cause of all this confusion? Curr Opin Crit Care, 2012. 18(5): p. 518-26.

96. Alexander, J.J., et al., TNF is a key mediator of septic encephalopathy acting through its receptor, TNF receptor-1. Neurochem Int, 2008. 52(3): p. 447-56.

97. Heneka, M.T., et al., Induction of nitric oxide synthase and nitric oxide-mediated apoptosis in neuronal PC12 cells after stimulation with tumor necrosis factoralpha/lipopolysaccharide. J Neurochem, 1998. 71(1): p. 88-94.

98. Leist, M., et al., Caspase-mediated apoptosis in neuronal excitotoxicity triggered by nitric oxide. Mol Med, 1997. 3(11): p. 750-64.

99. Sharshar, T., et al., Apoptosis of neurons in cardiovascular autonomic centres triggered by inducible nitric oxide synthase after death from septic shock. Lancet, 2003. 362(9398): p. 1799-805.

100. Takeshita, Y. and R.M. Ransohoff, Inflammatory cell trafficking across the blood-brain barrier: chemokine regulation and in vitro models. Immunol Rev, 2012. 248(1): p. 22839.

101. Wilson, E.H., W. Weninger, and C.A. Hunter, Trafficking of immune cells in the central nervous system. J Clin Invest, 2010. 120(5): p. 1368-79.

102. Kuperberg, S.J. and R. Wadgaonkar, Sepsis-Associated Encephalopathy: The BloodBrain Barrier and the Sphingolipid Rheostat. Front Immunol, 2017. 8: p. 597.

103. Zielinska, K.A., et al., Endothelial Response to Glucocorticoids in Inflammatory Diseases. Front Immunol, 2016. 7: p. 592.

104. Minagar, A., et al., Serum from patients with multiple sclerosis downregulates occludin and VE-cadherin expression in cultured endothelial cells. Mult Scler, 2003. 9(3): p. 2358.

105. Shimizu, F., et al., Sera from remitting and secondary progressive multiple sclerosis patients disrupt the blood-brain barrier. PLoS One, 2014. 9(3): p. e92872.

106. Rochfort, K.D., et al., Downregulation of blood-brain barrier phenotype by proinflammatory cytokines involves NADPH oxidase-dependent ROS generation: consequences for interendothelial adherens and tight junctions. PLoS One, 2014. 9(7): p. e101815.

107. Goncalves, A., A.F. Ambrosio, and R. Fernandes, Regulation of claudins in blood-tissue barriers under physiological and pathological states. Tissue Barriers, 2013. 1(3): p. e24782.

108. Ortiz, G.G., et al., Role of the blood-brain barrier in multiple sclerosis. Arch Med Res, 2014. 45(8): p. 687-97.

109. Alvarez, J.I., R. Cayrol, and A. Prat, Disruption of central nervous system barriers in multiple sclerosis. Biochim Biophys Acta, 2011. 1812(2): p. 252-64.

110. Liu, J., et al., Matrix metalloproteinase-2-mediated occludin degradation and caveolin-1mediated claudin-5 redistribution contribute to blood-brain barrier damage in early ischemic stroke stage. J Neurosci, 2012. 32(9): p. 3044-57.

111. Sowa, G., Role of Caveolin Proteins in Sepsis. Pediatr Ther, 2012. 2012(Suppl 2).

112. Wang, X., et al., Traumatic brain injury research and expression of caveolin-1 and its relationship with disease prognosis. Pak J Pharm Sci, 2017. 30(3(Suppl.)): p. 997-1000.

113. Wu, H., et al., Caveolin-1 Is Critical for Lymphocyte Trafficking into Central Nervous System during Experimental Autoimmune Encephalomyelitis. J Neurosci, 2016. 36(19): p. 5193-9.

114. Lopes Pinheiro, M.A., et al., Acid Sphingomyelinase-Derived Ceramide Regulates ICAM1 Function during $T$ Cell Transmigration across Brain Endothelial Cells. J Immunol, 2016. 196(1): p. 72-9. 
115. Allingham, M.J., J.D. van Buul, and K. Burridge, ICAM-1-Mediated, Src- and Pyk2Dependent Vascular Endothelial Cadherin Tyrosine Phosphorylation Is Required for Leukocyte Transendothelial Migration. The Journal of Immunology, 2007. 179(6): p. 4053-4064.

116. Zimmermann, J., et al., CNS-targeted production of IL-17A induces glial activation, microvascular pathology and enhances the neuroinflammatory response to systemic endotoxemia. PLoS One, 2013. 8(2): p. e57307.

117. Xie, L. and S.H. Yang, Interaction of astrocytes and T cells in physiological and pathological conditions. Brain Res, 2015. 1623: p. 63-73.

118. Crisafulli, S.G., et al., Therapeutic Strategies Under Development Targeting Inflammatory Mechanisms in Amyotrophic Lateral Sclerosis. Mol Neurobiol, 2017.

119. Deracinois, B., et al., Tissue Non-specific Alkaline Phosphatase (TNAP) in Vessels of the Brain. Subcell Biochem, 2015. 76: p. 125-51.

120. McEwen, B.S., et al., Putative estrogen and glucocorticoid receptors in the limbic brain. J Steroid Biochem, 1975. 6(6): p. 971-7.

121. Buchet, R., J.L. Millan, and D. Magne, Multisystemic functions of alkaline phosphatases. Methods Mol Biol, 2013. 1053: p. 27-51.

122. Low, M.G., Biochemistry of the glycosyl-phosphatidylinositol membrane protein anchors. Biochem J, 1987. 244(1): p. 1-13.

123. Low, M.G. and D.B. Zilversmit, Role of phosphatidylinositol in attachment of alkaline phosphatase to membranes. Biochemistry, 1980. 19(17): p. 3913-8.

124. Betz, A.L., J.A. Firth, and G.W. Goldstein, Polarity of the blood-brain barrier: distribution of enzymes between the luminal and antiluminal membranes of brain capillary endothelial cells. Brain Res, 1980. 192(1): p. 17-28.

125. Whyte, M.P., et al., Alkaline phosphatase: placental and tissue-nonspecific isoenzymes hydrolyze phosphoethanolamine, inorganic pyrophosphate, and pyridoxal 5'-phosphate. Substrate accumulation in carriers of hypophosphatasia corrects during pregnancy. J Clin Invest, 1995. 95(4): p. 1440-5.

126. Say, J.C., et al., Alkaline phosphatase from rat osseous plates: purification and biochemical characterization of a soluble form. Biochim Biophys Acta, 1991. 1074(2): p. 256-62.

127. Moore, C.A., et al., Infantile hypophosphatasia: autosomal recessive transmission to two related sibships. Am J Med Genet, 1990. 36(1): p. 15-22.

128. Barvencik, F., et al., Skeletal mineralization defects in adult hypophosphatasia--a clinical and histological analysis. Osteoporos Int, 2011. 22(10): p. 2667-75.

129. Fedde, K.N., et al., Alkaline phosphatase knock-out mice recapitulate the metabolic and skeletal defects of infantile hypophosphatasia. J Bone Miner Res, 1999. 14(12): p. 201526.

130. Whyte, M.P., Hypophosphatasia and the role of alkaline phosphatase in skeletal mineralization. Endocr Rev, 1994. 15(4): p. 439-61.

131. Orimo, $\mathrm{H}$., The mechanism of mineralization and the role of alkaline phosphatase in health and disease. J Nippon Med Sch, 2010. 77(1): p. 4-12.

132. Whyte, M.P., Physiological role of alkaline phosphatase explored in hypophosphatasia. Ann N Y Acad Sci, 2010. 1192: p. 190-200.

133. Waymire, K.G., et al., Mice lacking tissue non-specific alkaline phosphatase die from seizures due to defective metabolism of vitamin B-6. Nat Genet, 1995. 11(1): p. 45-51.

134. Whyte, M.P., et al., Markedly increased circulating pyridoxal-5'-phosphate levels in hypophosphatasia. Alkaline phosphatase acts in vitamin B6 metabolism. J Clin Invest, 1985. 76(2): p. 752-6.

135. Brun-Heath, I., et al., Differential expression of the bone and the liver tissue non-specific alkaline phosphatase isoforms in brain tissues. Cell Tissue Res, 2011. 343(3): p. 521-36. 
136. Anstrom, J.A., et al., Temporal expression pattern of cerebrovascular endothelial cell alkaline phosphatase during human gestation. J Neuropathol Exp Neurol, 2002. 61(1): p. 76-84.

137. Shimizu, N., Histochemical studies on the phosphatase of the nervous system. J Comp Neurol, 1950. 93(2): p. 201-17.

138. Fonta, C. and M. Imbert, Vascularization in the primate visual cortex during development. Cereb Cortex, 2002. 12(2): p. 199-211.

139. Bell, M.A. and W.G. Scarrow, Staining for microvascular alkaline phosphatase in thick celloidin sections of nervous tissue: morphometric and pathological applications. Microvasc Res, 1984. 27(2): p. 189-203.

140. Norman, M.G. and J.R. O'Kusky, The growth and development of microvasculature in human cerebral cortex. J Neuropathol Exp Neurol, 1986. 45(3): p. 222-32.

141. Latker, C.H., et al., Differential localization of alkaline phosphatase in barrier tissues of the frog and rat nervous systems: a cytochemical and biochemical study. J Comp Neurol, 1987. 264(3): p. 291-302.

142. Mizuguchi, H., et al., A comparison of drug transport through cultured monolayers of bovine brain capillary and bovine aortic endothelial cells. Biol Pharm Bull, 1994. 17(10): p. $1385-90$.

143. Vorbrodt, A.W., A.S. Lossinsky, and H.M. Wisniewski, Localization of alkaline phosphatase activity in endothelia of developing and mature mouse blood-brain barrier. Dev Neurosci, 1986. 8(1): p. 1-13.

144. M.J. Weiss, K.R., P.S. Henthorn, B. Lamb, T. Kadesch, H. Harris, Structure of the human liver/bone/kidney alkaline phosphatase gene. JOURNAL OF BIOLOGICAL CHEMISTRY, 1988. 263: p. 12002-10.

145. Toh, Y., et al., Isolation and characterization of a rat liver alkaline phosphatase gene. $A$ single gene with two promoters. Eur J Biochem, 1989. 182(2): p. 231-7.

146. Studer, M., et al., Characterization of a second promoter for the mouse liver/bone/kidney-type alkaline phosphatase gene: cell and tissue specific expression. Biochem Biophys Res Commun, 1991. 179(3): p. 1352-60.

147. Matsuura, S., F. Kishi, and T. Kajii, Characterization of a 5'-flanking region of the human liver/bone/kidney alkaline phosphatase gene: two kinds of mRNA from a single gene. Biochem Biophys Res Commun, 1990. 168(3): p. 993-1000.

148. Hahnel, A.C., et al., Two alkaline phosphatase genes are expressed during early development in the mouse embryo. Development, 1990. 110(2): p. 555-64.

149. Narisawa, S., N. Frohlander, and J.L. Millan, Inactivation of two mouse alkaline phosphatase genes and establishment of a model of infantile hypophosphatasia. Dev Dyn, 1997. 208(3): p. 432-46.

150. Kruse, K., et al., Hyperphosphatasia with mental retardation. J Pediatr, 1988. 112(3): p. 436-9.

151. Thompson, M.D., et al., Hyperphosphatasia with seizures, neurologic deficit, and characteristic facial features: Five new patients with Mabry syndrome. Am J Med Genet A, 2010. 152A(7): p. 1661-9.

152. Thompson, M.D., D.E. Cole, and C.C. Mabry, 50 Years Ago in TheJournal ofPediatrics: Familial Hyperphosphatasia with Mental Retardation, Seizures, and Neurologic Deficits. J Pediatr, 2020. 222: p. 97.

153. Liedtke, D., et al., Tissue-Nonspecific Alkaline Phosphatase-A Gatekeeper of Physiological Conditions in Health and a Modulator of Biological Environments in Disease. Biomolecules, 2020. 10(12).

154. Narisawa, S., Genetically Modified Mice for Studying TNAP Function. Subcell Biochem, 2015. 76: p. 45-57. 
155. Liu, J., et al., Tissue-nonspecific alkaline phosphatase deficiency causes abnormal craniofacial bone development in the Alpl(-/-) mouse model of infantile hypophosphatasia. Bone, 2014. 67: p. 81-94.

156. Sebastian-Serrano, A., et al., Haploinsufficient TNAP Mice Display Decreased Extracellular ATP Levels and Expression of Pannexin-1 Channels. Front Pharmacol, 2018. 9: p. 170.

157. Gamez-Belmonte, R., et al., Deficiency in Tissue Non-Specific Alkaline Phosphatase Leads to Steatohepatitis in Mice Fed a High Fat Diet Similar to That Produced by a Methionine and Choline Deficient Diet. Int J Mol Sci, 2020. 22(1).

158. Hernandez-Chirlaque, C., et al., Tissue Non-specific Alkaline Phosphatase Expression is Needed for the Full Stimulation of T Cells and T Cell-Dependent Colitis. J Crohns Colitis, 2017. 11(7): p. 857-870.

159. Yamamoto, S., et al., Prolonged survival and phenotypic correction of Akp2(-/-) hypophosphatasia mice by lentiviral gene therapy. J Bone Miner Res, 2011. 26(1): p. 135-42.

160. Nam, H.K., et al., Viral delivery of tissue nonspecific alkaline phosphatase diminishes craniosynostosis in one of two FGFR2C342Y/+ mouse models of Crouzon syndrome. PLoS One, 2020. 15(5): p. e0234073.

161. Arno, B., et al., TNAP limits TGF-beta-dependent cardiac and skeletal muscle fibrosis by inactivating the SMAD2/3 transcription factors. J Cell Sci, 2019. 132(15).

162. Sheen, C.R., et al., Pathophysiological role of vascular smooth muscle alkaline phosphatase in medial artery calcification. J Bone Miner Res, 2015. 30(5): p. 824-36.

163. Foster, B.L., et al., Conditional Alpl Ablation Phenocopies Dental Defects of Hypophosphatasia. J Dent Res, 2017. 96(1): p. 81-91.

164. Rufo, M.B. and W.H. Fishman, L-homoarginine, a specific inhibitor of liver-type alkaline phosphatase, applied to the recognition of liver-type enzyme activity in rat intestine. $\mathrm{J}$ Histochem Cytochem, 1972. 20(5): p. 336-43.

165. Kozlenkov, A., et al., Residues determining the binding specificity of uncompetitive inhibitors to tissue-nonspecific alkaline phosphatase. J Bone Miner Res, 2004. 19(11): p. 1862-72.

166. Rodionov, R.N., et al., Homoarginine Supplementation Prevents Left Ventricular Dilatation and Preserves Systolic Function in a Model of Coronary Artery Disease. J Am Heart Assoc, 2019. 8(14): p. e012486.

167. Diez-Zaera, M., et al., Tissue-nonspecific alkaline phosphatase promotes axonal growth of hippocampal neurons. Mol Biol Cell, 2011. 22(7): p. 1014-24.

168. Deracinois, B., et al., TNAP and EHD1 are over-expressed in bovine brain capillary endothelial cells after the re-induction of blood-brain barrier properties. PLoS One, 2012. 7(10): p. e48428.

169. Nakamura, T., et al., Tissue-nonspecific alkaline phosphatase promotes the osteogenic differentiation of osteoprogenitor cells. Biochem Biophys Res Commun, 2020. 524(3): p. 702-709.

170. Dahl, R., et al., Discovery and validation of a series of aryl sulfonamides as selective inhibitors of tissue-nonspecific alkaline phosphatase (TNAP). J Med Chem, 2009. 52(21): p. 6919-25.

171. Pinkerton, A.B., et al., Discovery of 5-((5-chloro-2methoxyphenyl)sulfonamido)nicotinamide (SBI-425), a potent and orally bioavailable tissue-nonspecific alkaline phosphatase (TNAP) inhibitor. Bioorg Med Chem Lett, 2018. 28(1): p. 31-34.

172. Romanelli, F., et al., Overexpression of tissue-nonspecific alkaline phosphatase (TNAP) in endothelial cells accelerates coronary artery disease in a mouse model of familial hypercholesterolemia. PLoS One, 2017. 12(10): p. e0186426. 
173. Opdebeeck, B., et al., Pharmacological TNAP inhibition efficiently inhibits arterial media calcification in a warfarin rat model but deserves careful consideration of potential physiological bone formation/mineralization impairment. Bone, 2020. 137: p. 115392.

174. Brichacek, A.L., et al., Systemic inhibition of tissue-nonspecific alkaline phosphatase alters the brain-immune axis in experimental sepsis. Sci Rep, 2019. 9(1): p. 18788.

175. Nwafor, D.C., et al., Targeting the Blood-Brain Barrier to Prevent Sepsis-Associated Cognitive Impairment. J Cent Nerv Syst Dis, 2019. 11: p. 1179573519840652.

176. Hu, Y., et al., Omi/HtrA2 Protease Associated Cell Apoptosis Participates in Blood-Brain Barrier Dysfunction. Front Mol Neurosci, 2019. 12: p. 48.

177. Nation, D.A., et al., Blood-brain barrier breakdown is an early biomarker of human cognitive dysfunction. Nat Med, 2019. 25(2): p. 270-276.

178. Vorbrodt, A.W., et al., Ultrastructural cytochemical studies of cerebral microvasculature in scrapie infected mice. Acta Neuropathol, 1981. 53(3): p. 203-11.

179. Zoellner, H.F. and N. Hunter, Histochemical identification of the vascular endothelial isoenzyme of alkaline phosphatase. J Histochem Cytochem, 1989. 37(12): p. 1893-8.

180. Mayahara, H., et al., The new lead citrate method for the ultracytochemical demonstration of activity of non-specific alkaline phosphatase (orthophosphoric monoester phosphohydrolase). Histochemie, 1967. 11(1): p. 88-96.

181. Mori, S. and M. Nagano, Electron-microscopic cytochemistry of alkaline-phosphatase activity in endothelium, pericytes and oligodendrocytes in the rat brain. Histochemistry, 1985. 82(3): p. 225-31.

182. Ovtscharoff, W., [Ultracytochemical localisation of the alkaline phosphatase in the cerebral cortex of newborn rats (author's transl)]. Histochemie, 1973. 37(1): p. 93-5.

183. Vincent, J.L., et al., Use of the SOFA score to assess the incidence of organ dysfunction/failure in intensive care units: results of a multicenter, prospective study. Working group on "sepsis-related problems" of the European Society of Intensive Care Medicine. Crit Care Med, 1998. 26(11): p. 1793-800.

184. Seymour, C.W., et al., Assessment of Clinical Criteria for Sepsis: For the Third International Consensus Definitions for Sepsis and Septic Shock (Sepsis-3). JAMA, 2016. 315(8): p. 762-74.

185. Hollinger, P., et al., Diffusion magnetic resonance imaging findings in cerebritis and brain abscesses in a patient with septic encephalopathy. J Neurol, 2000. 247(3): p. 232-4.

186. Sharshar, T., et al., Brain lesions in septic shock: a magnetic resonance imaging study. Intensive Care Med, 2007. 33(5): p. 798-806.

187. Jackson, J.C., et al., Acute respiratory distress syndrome, sepsis, and cognitive decline: a review and case study. South Med J, 2009. 102(11): p. 1150-7.

188. Kondo, A., et al., Fulminant sepsis-associated encephalopathy in two children: serial neuroimaging findings and clinical course. Neuropediatrics, 2009. 40(4): p. 157-61.

189. Morandi, A., et al., Neuroimaging in delirious intensive care unit patients: a preliminary case series report. Psychiatry (Edgmont), 2010. 7(9): p. 28-33.

190. Bozza, F.A., et al., Sepsis-associated encephalopathy: a magnetic resonance imaging and spectroscopy study. J Cereb Blood Flow Metab, 2010. 30(2): p. 440-8.

191. Suchyta, M.R., A. Jephson, and R.O. Hopkins, Neurologic changes during critical illness: brain imaging findings and neurobehavioral outcomes. Brain Imaging Behav, 2010. 4(1): p. 22-34.

192. Luitse, M.J., C.J. van Asch, and C.J. Klijn, Deep coma and diffuse white matter abnormalities caused by sepsis-associated encephalopathy. Lancet, 2013. 381(9884): p. 2222. 


\title{
Chapter 2
}

\section{Loss of tissue-nonspecific alkaline phosphatase (TNAP) enzyme activity in cerebral microvessels is coupled to persistent neuroinflammation and behavioral deficits in late sepsis}

\author{
Divine C. Nwafor ${ }^{a}$, Sreeparna Chakraborty ${ }^{b}$, Allison L. Brichacek ${ }^{b}$, Sujung Jun ${ }^{c}$, Catheryne A. \\ Gambill $^{b}$, Wei Wang ${ }^{a}$, Elizabeth B. Engler-Chiurazzi ${ }^{a}$, Duaa Dakhlallah ${ }^{a, d}$, Anthony B. Pinkerton ${ }^{e}$, \\ José Luis Millán ${ }^{\mathrm{e}}$, Stanley A. Benkovic ${ }^{\mathrm{a}}$, and Candice M. Brown ${ }^{\mathrm{a}, \mathrm{b}^{*}}$ \\ aDepartment of Neuroscience, West Virginia University Health Science Center, Morgantown, \\ WV 26506, USA \\ ${ }^{b}$ Department of Microbiology, Immunology, and Cell Biology, School of Medicine, West Virginia \\ University Health Science Center, Morgantown, WV 26506, USA \\ 'Wilmer Eye Institute, John Hopkins University School of Medicine, Baltimore, MD, 21231, USA \\ ${ }^{\mathrm{d}}$ Cancer Institute, West Virginia University Health Science Center, Morgantown, WV 26506, \\ USA \\ 'Sanford-Burnham Prebys Medical Discovery Institute, La Jolla, CA, 92037, USA
}

Nwafor DC, Chakraborty S, Brichacek AL, Jun S, Gambill CA, Wang W, Engler-Chiurazzi EB, Dakhlallah D, Pinkerton AB, Millán JL, Benkovic SA, Brown CM. Loss of tissue-nonspecific alkaline phosphatase (TNAP) enzyme activity in cerebral microvessels is coupled to persistent neuroinflammation and behavioral deficits in late sepsis. Brain Behav Immun. 2020 Feb; 84:115-131. doi: 10.1016/j.bbi.2019.11.016. Epub 2019 Nov 25. PMID: 31778743; PMCID: PMC7010562.

Nwafor DC, Brichacek AL, Mohammad AS, Griffith J, Lucke-Wold BP, Benkovic SA, Geldenhuys WJ, Lockman PR, Brown CM. Targeting the Blood-Brain Barrier to Prevent SepsisAssociated Cognitive Impairment. J Cent Nerv Syst Dis. 2019 Apr 9; 11:1179573519840652. doi: 10.1177/1179573519840652. PMID: 31007531; PMCID: PMC6456845.

Note: Parts of this chapter include text and figures taken from the publications highlighted above 


\section{Highlights}

- Alkaline phosphatase activity in brain microvessels is diminished in early and late sepsis.

- Loss of alkaline phosphatase activity is coupled to the loss of barrier integrity.

- Brain infiltration of myeloid and T-lymphoid cells persists in late sepsis.

- Septic mice exhibit a novel anti-nociceptive phenotype.

- Cortical astrogliosis and microgliosis persist in late sepsis. 


\subsection{Abstract}

Sepsis is a host response to systemic inflammation and infection that may lead to multiorgan dysfunction and eventual death. While acute brain dysfunction is common among all sepsis patients, chronic neurological impairment is prevalent among sepsis survivors. The brain microvasculature has emerged as a major determinant of sepsis-associated brain dysfunction, yet the mechanisms that underlie its associated neuroimmune perturbations and behavioral deficits are not well understood. An emerging body of data suggests that inhibition of tissuenonspecific alkaline phosphatase (TNAP) enzyme activity in cerebral microvessels may be associated with changes in endothelial cell barrier integrity. The objective of this study was to elucidate the connection between alterations in cerebrovascular TNAP enzyme activity and brain microvascular dysfunction in late sepsis. We hypothesized that the disruption of TNAP enzymatic activity in cerebral microvessels would be coupled to the sustained loss of brain microvascular integrity, elevated neuroinflammatory responses, and behavioral deficits. Male mice were subjected to cecal ligation and puncture (CLP), a model of experimental sepsis, and assessed up to seven days post-sepsis. All mice were observed daily for sickness behavior and underwent behavioral testing. Our results showed a significant decrease in brain microvascular TNAP enzyme activity in the somatosensory cortex and spinal cord of septic mice but not in the CA1 and CA3 hippocampal regions. Furthermore, we showed that loss of cerebrovascular TNAP enzyme activity was coupled to a loss of claudin-5 and increased perivascular IgG infiltration in the somatosensory cortex. Analyses of whole brain myeloid and T-lymphoid cell populations also revealed a persistent elevation of infiltrating leukocytes, which included both neutrophil and monocyte myeloid derived suppressor cells (MDSCs). Regional analyses of the somatosensory cortex, hippocampus, and spinal cord revealed significant astrogliosis and microgliosis in the cortex and spinal cord of septic mice that was accompanied by significant microgliosis in the CA1 and CA3 hippocampal regions. Assessment of behavioral deficits revealed no changes in learning and memory or evoked locomotion. However, the hot plate test 
uncovered a novel anti-nociceptive phenotype in our septic mice, and we speculate that this phenotype may be a consequence of sustained GFAP astrogliosis and loss of TNAP activity in the somatosensory cortex and spinal cord of septic mice. Taken together, these results demonstrate that the loss of TNAP enzyme activity in cerebral microvessels during late sepsis is coupled to sustained neuroimmune dysfunction which may underlie, in part, the chronic neurological impairments observed in sepsis survivors.

Keywords: sepsis; cecal ligation and puncture; tissue non-specific alkaline phosphatase, bloodbrain barrier; nociception; astrogliosis; microgliosis; neuroimmune 


\subsection{Introduction}

Sepsis is a potentially fatal clinical syndrome that results from an excessive systemic inflammatory response to an infection [1-3]. This syndrome accounts for 30 million cases annually, and about 6 million deaths worldwide [4]. A unique feature of sepsis is that it is often comorbid or may precipitate from other disease conditions such as stroke, cancer, or diabetes [5-7]. It is anticipated that the rise in these chronic diseases will increase the global prevalence of sepsis $[8,9]$. Yet, therapeutic strategies to treat septic patients remain limited, as there are no FDA-approved drugs to treat sepsis, and the primary treatments are largely limited to antimicrobial drugs, supportive fluids, and vasopressors [10]. In addition, the mechanisms underlying the long-term consequences of sepsis on the brain and periphery remain elusive [11, 12].

Sepsis survivors are burdened with an increased risk of infections, sensorimotor abnormalities, and cognitive decline $[5,12,13]$. The central nervous system (CNS), particularly the brain, is severely affected in sepsis and sepsis-associated cognitive impairment is an independent predictor of mortality [14]. Persistent peripheral inflammation in sepsis contributes to a dysfunctional brain microvasculature and facilitates the trafficking of peripheral immune cell populations, cytokines and neurotoxins into the brain which, collectively, promote neuroinflammation and alter brain function $[15,16]$. Short-term brain microvascular dysfunction in murine models of experimental sepsis has been demonstrated by increased barrier permeability and diminished tight junction [17] proteins $24 \mathrm{~h}$ post-sepsis [18, 19]. Thus, a better understanding of the cellular and molecular mechanisms that contribute to brain microvascular dysfunction in sepsis may provide insights to mitigate and improve long term neurological outcomes in sepsis survivors.

We and others have shown that the ectoenzyme tissue-nonspecific alkaline phosphatase (TNAP) may play a critical role in brain microvascular function and dysfunction [5, 20]. TNAP, also known as bone/liver/kidney alkaline phosphatase, is one of four alkaline 
phosphatase genes in the mammalian genome [21]. Peripheral TNAP enzymatic activity, most likely generated within neutrophils, liver, and kidneys, has been shown to play an important role in attenuating inflammation and improving survival in sepsis rodent models $[22,23]$. TNAP enzyme activity is also highly elevated in cerebral microvessels and has been used as a histological marker of the brain microvasculature for nearly a century [24-26]. While a clearly delineated physiological role for TNAP at the brain microvascular interface remains less understood, recent studies suggest a role for TNAP in the maintenance of brain endothelial cell integrity. An in vitro study demonstrated that inhibition of TNAP activity on brain endothelial cells by levamisole, a pan-AP enzyme inhibitor, worsened barrier function and increased cellular permeability [20]. In a recent in vivo study, we reported that brain TNAP enzyme activity is decreased $24 \mathrm{~h}$ post-sepsis compared to sham-injured mice [5]. Taken together, both findings suggest a plausible role for TNAP in maintaining brain microvascular barrier integrity during inflammation. However, it remains unclear whether the observed decrease in TNAP's enzyme activity persists beyond 24 hours and whether the decrease in TNAP enzyme activity is coupled to brain microvascular dysfunction in vivo. The delineation between early $(\leq 24 \mathrm{~h})$ and late sepsis $(>36 \mathrm{~h})$ is particularly important considering that most sepsis-related deaths occur in the late/hypo-inflammatory phase of sepsis [27].

The objective of this study was to determine the neuroimmune and behavioral changes which paralleled the loss of TNAP in cerebral microvessels in late sepsis, i.e., up to seven days post-sepsis. We incorporated an established a seven-day model of late sepsis, cecal ligation and puncture (CLP) [28-30]. We assessed novel behavioral and neuroinflammatory outcomes in late sepsis and coupled these outcomes to distinct alterations in the brain microvasculature and integrity. Our results revealed a loss of TNAP enzymatic activity in cerebral microvessels, diminished tight junctions and increased permeability, indicating a loss of barrier integrity. Enhanced cortical astrogliosis and microgliosis was accompanied by sustained leukocyte infiltration, and we also identified a novel cell population infiltrate with phenotypic characteristics 
of myeloid-derived suppressor cells (MDSCs). Assessment of behavioral deficits in late sepsis uncovered a novel anti-nociceptive phenotype in the absence of an impairment in learning and memory. Taken together, these results demonstrate that late sepsis is characterized by a novel neuroimmune phenotype that embodies loss of sustained loss of cerebral microvascular TNAP activity and persistent neuroinflammation.

\subsection{Materials and Methods}

\subsubsection{Animals}

All experiments were conducted in accordance with the National Institutes of Health Guide for the Care and Use of Laboratory Animals and were approved by the Institutional Animal Care and Use Committee at West Virginia University. Male wild-type (WT; C57BL6/J) mice were bred in West Virginia University Health Sciences Center vivarium facilities and used for experiments at 3-5 months old. All mice were generated from C57BL/6J breeding pairs obtained from Jackson Labs (Bar Harbor, ME, Catalog \# 000664). Mice were group housed in environmentally controlled conditions with a reverse light cycle $(12: 12 \mathrm{~h}$ light/dark cycle at $21 \pm 1^{\circ} \mathrm{C}$ ) and provided food and water ad libitum.

\subsubsection{SBI-425 preparation}

5-((5-chloro-2-methoxyphenyl) sulfonamido) nicotinamide (SBI-425) was synthesized as previously described [31]. SBI-425 powder was dissolved in 100\% dimethyl sulfoxide (DMSO, Sigma-Aldrich, Milwaukee, $\mathrm{WI})$, heated, sonicated, and stored at $-80^{\circ} \mathrm{C}$. The stock solution was diluted to $1 \mathrm{mg} / \mathrm{mL}$ in a vehicle solution comprised of: $10 \%$ DMSO, 10\% Tween-80 (SigmaAldrich, Milwaukee, $\mathrm{WI})$, and $80 \%$ water.

\subsubsection{Brain endothelial cell barrier function assays}


The human cerebral microvascular endothelial cell line (hCMEC/D3) is an immortalized line that retains BBB characteristics in vitro [32]. hCMEC/D3 cells were seeded onto 3 independent collagen-coated 16-well E-Plate PET arrays (ACEA Biosciences, San Diego, CA) at a concentration of 20,000 cells/well and loaded onto an xCelligence RTCA DP system (ACEA Biosciences) enclosed in a tissue culture incubator. Once cells reached confluence $\sim 24 \mathrm{~h}$ after seeding, duplicate wells in each array were treated with $200 \mu$ of the following: vehicle (DMSO, 0.3\%), tissue-nonspecific alkaline phosphatase inhibitor (TNAPi $100 \mu \mathrm{M}$; Millipore, Temecula, CA), lipopolysaccharide (LPS $100 \mathrm{ng} / \mathrm{mL}$; Sigma-Aldrich, Milwaukee, WI), or LPS (100 ng/ml) and TNAPi $(100 \mu \mathrm{M})$. Cell index [20] was recorded continuously for $24 \mathrm{~h}$ and analyzed with RTCA Software 2.0 (ACEA Biosciences). Cl for all wells was normalized to a single timepoint, i.e., $\sim 1 \mathrm{~h}$ after treatment and reported as normalized $\mathrm{Cl}$.

\subsubsection{Cecal ligation and puncture (CLP)}

The cecal ligation and puncture (CLP) model of moderate polymicrobial sepsis was employed as previously described [30]. Briefly, mice were anesthetized by the inhalation of 1 $2 \%$ isoflurane. Abdominal access was obtained via a midline incision following topical application of $2 \%$ lidocaine (Hospira, Lake Forest, IL). The cecum was isolated and ligated with a 4-0 silk ligature below the ileocecal valve and then punctured twice with a $22 \mathrm{G}$ needle through and through. Fecal matter (approximately $1 \mathrm{~mm}$ ) was extruded from the hole, and the cecum was placed back into the abdominal cavity. The abdominal muscle layer was closed with 6-0 sutures (Ethilon, Cornelia, GA), while the abdominal skin layer was closed with 5-0 sutures (Ethilon, Cornelia, GA). Sham-operated animals had their cecum isolated and then returned to the peritoneal cavity without being ligated or punctured. One $\mathrm{mL}$ of sterile $0.9 \%$ saline was administered subcutaneously after sepsis procedure for fluid resuscitation in sham- and CLPoperated mice. All mice were housed according to treatment group and placed on heating pads 
for three days following sepsis procedure. Mice were monitored for survival and sepsisassociated clinical signs twice daily for six days and underwent a series of behavioral assessments. To assess the role of TNAP in maintaining brain endothelial cell barrier integrity in vivo, a third cohort of septic mice was used for SBI-425 studies. Briefly, mice were injected intraperitoneally with $25 \mathrm{mg} / \mathrm{kg} \mathrm{SBI}-425$ or vehicle $1 \mathrm{~h}$ following surgery and once daily for the next six days; this dosage was derived from a previously published study [33]. Mice used for all experiments were euthanized at day seven post-sepsis.

\subsubsection{Modified murine sepsis score (MMSS) and average weight loss score}

We modified a validated murine sepsis severity score (MSS) [34] to examine eight sickness behavior components: appearance, weight loss, activity, response to stimulus, eyes, posture, diarrhea, and respiratory rate, henceforth referred to as the modified murine sepsis severity score (MMSS). Each component is scored from 0 (normal/no change) to 4 (severe change), and the MMSS is a grand total of all eight aforementioned sickness behavior components (i.e., 32 total points) collected each day post-sepsis over a 6-day period for each animal. The average percent weight loss score was derived from the MMSS and defined on a scale from 0 to 4 (i.e., a score of $0=0-5 \%, 1=5.1-10 \%, 2=10.1-15 \%, 3=15.1-20 \%$, and $4=>20.1 \%$ average percent weight loss). A score of 24 or greater on the MMSS represents a humane endpoint for all animals used in our studies. Animals with a MMSS greater than 24 were euthanized and excluded from behavioral assessments.

\subsubsection{Tissue collecting and processing}

Mice were deeply anesthetized with isoflurane and perfused intracardially with a perfusion pump (Masterflex 7524-10, Cole-Parmer, Vernon Hills, IL) set to $5.0 \mathrm{~mL} / \mathrm{min}$. Blood was removed with $25 \mathrm{~mL}$ of $0.9 \%$ saline followed by perfusion and fixation with $50 \mathrm{~mL} 4 \%$ chilled paraformaldehyde (PFA, Fisher Scientific, Pittsburgh, PA). Perfused brains and spinal 
cords were removed from the skull and spinal canal, respectively, and post-fixed in $4 \%$ PFA overnight at $4^{\circ} \mathrm{C}$. On the following day, brains and spinal cords were rinsed in $0.01 \mathrm{M}$ phosphate buffered saline (PBS) and incubated sequentially in 10\%, 20\%, and 30\% sucrose in PBS for 24 h each. Following successive incubations in sucrose, brains and spinal cords were coembedded in a $15 \%$ gelatin matrix in groups of twelve per matrix for simultaneous sectioning. The gelatin block was processed sequentially through 4\% PFA for $24 \mathrm{~h}, 15 \%$ sucrose for $24 \mathrm{~h}$, and $30 \%$ sucrose for $48 \mathrm{~h}$. The block was trimmed and placed in a $-80^{\circ} \mathrm{C}$ freezer for $30 \mathrm{~min}$. Sectioning was performed in the coronal plane at $30 \mu \mathrm{m}$ on a sliding microtome (HM 450, ThermoFisher Scientific, Waltham, MA) equipped with a 3×3 freezing stage (BFS-40MPA, Physitemp, Clifton, NJ) at $-20^{\circ} \mathrm{C}$. Sections were collected into a series of six cups filled with PBS/0.06\% sodium azide. Adjacent cups were used for sequential histological staining or immunostaining.

\subsubsection{Immunohistochemistry}

Following sequential cuts of the gelatin blocks on the microtome, sections were immunostained using standard free-floating immunohistochemistry techniques as described [35]. Briefly, all sections were blocked with $80 \mathrm{~mL}$ PBS, $10 \mathrm{~mL}$ of methanol (Fisher Scientific, Pittsburgh, PA), and $10 \mathrm{~mL}$ of $30 \%$ hydrogen peroxide (Fisher Scientific, Pittsburgh, PA) and incubated on a shaker (Model 55D, Reliable Scientific, Hernando, MS) for 15 min. Sections were then washed three times and permeabilized for 30 min on a shaker with $1.83 \%$ lysine (Fisher Scientific, Pittsburgh, PA) in 1\% Triton (Sigma-Aldrich, St. Louis, MO), and 4\% heatinactivated horse serum (Sigma-Aldrich, St. Louis, MO). Sections were then incubated for $24 \mathrm{~h}$ with primary antibodies at room temperature, followed by a $2 \mathrm{~h}$ incubation with the appropriate secondary antibody at room temperature. The following primary antibodies were used with working dilutions and antibody identification indicated in parentheses: Iba-1 (Invitrogen (1:1000), AB_2544912, Carlsbad, CA), GFAP (Agilent (1:10,000), AB_2811722, Santa Clara, CA), 
Claudin 5 (GeneTex (1:500), AB_2811720, Irvine, CA), TMEM 119 (Abcam (1:1000),

AB_2800343, Cambridge, MA), NeuN (Cell Signaling Technologies (1:500), AB_2651140, Danvers, MA), and choline acetyltransferase [36] antibody (Abcam (1:1000), AB_2721842, Cambridge, MA).

\subsubsection{Tissue non-specific alkaline phosphatase (TNAP) enzyme histology}

Brains and spinal cords and were evaluated for alkaline phosphatase enzyme activity with the BCIP/NBT substrate kit (SK-5400, Vector Laboratories, Burlingame, CA) as previously described [5]. Sections were rinsed in PBS twice for 5 min each, once in $0.1 \mathrm{M}$ Tris- $\mathrm{HCl}(\mathrm{pH}=$ 9.5) for $5 \mathrm{~min}$ and incubated in the staining solution for $4 \mathrm{~h}$ at room temperature. Following three rinses in 0.01 M PBS, the sections were mounted onto microscope slides (Unifrost+, Azer Scientific, Morgantown, PA), air-dried overnight, dehydrated through a standard dehydration series, and cover-slipped with Permount (Fisher Scientific, Pittsburgh, PA).

\subsubsection{Image analysis}

Sections were viewed on a Leica DM6B microscope (Leica Camera, Allendale, NJ) and images were captured using Leica LASX software (Leica Microsystems, Buffalo Grove, IL). All brain regions of interest i.e., the striatum, CA1, CA3, somatosensory cortex, and basal forebrain (diagonal band of Broca and medial septum) were identified by referring to the Allen Institute Brain Atlas (http://mouse.brain-map.org). Analysis of Iba-1 and TMEM 119 was conducted in the somatosensory cortex (20X (TMEM119), and 20X and 40X (Iba-1) magnifications), CA1 (40X (TMEM119 and Iba-1) magnification), and CA3 brain regions (40X (TMEM119 and lba-1) magnification). Analysis for GFAP was conducted in the somatosensory cortex (20X magnification), CA1 (40X magnification), and CA3 (40X magnification). Cell counting analysis for NeuN was conducted in the CA1 and CA3 hippocampal regions at 40X magnification. Cholinergic (ChAT-positive) neurons were analyzed in the basal forebrain region at 40X 
magnification. Analysis for TNAP enzyme activity was conducted in the somatosensory cortex at 20X magnification. Three random images were collected per animal per brain region of interest. Spinal cords were analyzed for GFAP, Iba-1, and TNAP. All collected images were converted to an 8-bit image and quantified in FIJI/Image $\mathrm{J}$ version 2.0 software.

\subsubsection{Flow cytometric sample preparation, gating strategy and analysis}

Single cell suspensions were obtained as previously described using the Miltenyi adult brain dissociation kit (Miltenyi Biotec, Auburn, CA) according to the manufacturer's instructions $[30,37]$. Briefly, mice were deeply anesthetized with isoflurane and were perfused intracardially with a perfusion pump (Masterflex 7524-10, Cole-Parmer, Vernon Hills, IL) set to $5.0 \mathrm{ml} / \mathrm{min}$. Blood was removed with $25 \mathrm{~mL}$ 0.9\% saline. Perfused whole brains were removed from the skull and processed with the Adult Brain Dissociation Kit (Miltenyi Biotec, Auburn, CA) according to manufacturer's instructions. Briefly, brains were minced followed by mechanical homogenization in a gentleMACS C tube with the gentleMACS Octo Dissociator (Miltenyi Biotec). Following centrifugation, cells were further dissociated using a $70 \mathrm{~m}$ strainer to obtain a single cell suspension. Myelin and other debris were removed, followed by red blood cell lysis with ACK Lysis Buffer (Lonza, Walkersville, MD). CD45+ cells within the suspension were isolated with mouse CD45 microbeads (Miltenyi Biotec) and magnetic separation with an autoMACS separator (Miltenyi Biotec). Viability and total cell yield were determined by trypan blue exclusion and cells were re-suspended to a final concentration of $2.5-3 \times 10^{6} \mathrm{cells} / \mathrm{ml}$ with FACS buffer (0.01 M PBS, 5mM EDTA, 2\% FBS). Cells were washed twice in cold 0.01M PBS and stained with fixable viability dye eFluor780 (eBioscience, San Diego, USA) for 30 min at $4^{\circ} \mathrm{C}$ in the dark. Cells were then briefly washed with FACS buffer and blocked with Ultra-Leaf purified anti-mouse CD16/32 (BioLegend, AB_2783156, San Diego, CA) for 20 min. Following non-specific blocking, cells were stained with monoclonal antibodies for CD45-PE (AB_2727965), CD11b-VioBlu (AB_2726322), CD11c-PerCp-Vio700 (AB_2660163), Ly6G-APC 
(AB_2660046), Ly6C-PE-Vio770 (AB_2660034) (Miltenyi Biotec, Auburn, CA) or CD4-FITC (AB_464896), CD25-PE (AB_465607), CD19-PerCp-Cy5.5 (AB_1106999) and CD8a-eFluor450 (AB_1272198) (eBioscience, San Diego, USA) for $10 \mathrm{~min}$ at $4^{\circ} \mathrm{C}$. Appropriate single stained controls were prepared for fluorophore compensation using compensation beads (Invitrogen, Carlsbad, CA). For intracellular FOXP3 detection cells were fixed/permeabilized using a mouse regulatory $T$ cell staining kit (eBioscience) after staining the surface markers (CD4 or CD25, Invitrogen, Carlsbad, CA) and stained with anti-FOXP3-PE-Cy5 (eBioscience, AB_468806, San Diego, USA). Fluorescence was measured using a BD LSR Fortessa with FACS Diva software (BD Biosciences, San Jose, CA). All data were compensated and spectral overlap was minimized using automatic compensation method of BD FACS diva software (BD Biosciences, San Jose, CA).

Further analyses were performed in FCS Express 6.0 (De Novo Software, Glendale, CA). Single cells were identified by forward scatter and side scatter, and viable cells were gated for further analysis of positive cell populations. Viable cells were gated for $\mathrm{CD}_{4} 5^{+}$populations and then divided into T-lymphoid cells, which included cytotoxic T-cells $\left(\mathrm{CD} 45^{\mathrm{hi}}, \mathrm{CD} 3^{+}, \mathrm{CD} 8^{+}\right)$, helper T-cells $\left(\mathrm{CD} 45^{\mathrm{hi}}, \mathrm{CD}^{+}, \mathrm{CD} 4^{+}\right)$, and T-regulatory cells $\left(\mathrm{CD} 4^{+}, \mathrm{CD} 25^{+}\right.$, Foxp3 $\left.3^{+}\right)$; myeloid cells, which included neutrophils $\left(C D 45^{\text {hi }}\right.$, CD11 b $b^{+}$, Ly6C $^{-}$, Ly6G $\left.^{+}\right)$, monocytes (CD45 ${ }^{\text {hi }}$, CD11C ${ }^{+}$, Ly6C $C^{+}$, Ly6G-), polymorphonuclear myeloid-derived suppressor cells (PMN-MDSCs) (CD45 ${ }^{\text {hi }}$, CD11b ${ }^{+}$, Ly6C ${ }^{\text {low }}$, Ly6G ${ }^{+}$), and monocytic myeloid-derived suppressor cells (M-MDSCs) (CD45 ${ }^{\text {hi }}$, CD11 $\left.\mathrm{b}^{+}, \mathrm{Ly}_{6 \mathrm{C}^{\mathrm{hi}}}, \mathrm{Ly}_{6 \mathrm{G}}\right)$ ). The quantified results represent the percentage of positive cells from the total $\mathrm{CD} 45^{+}$live cells.

\subsubsection{Behavioral testing}

\subsubsection{Open field test [38]}

The open field-testing paradigm was used to evaluate passive locomotor activity as previously described [39] and was administered on day two post-sepsis. A white plastic box 
$(60 \mathrm{~cm} \times 60 \mathrm{~cm} \times 15 \mathrm{~cm}$ ) was placed upon a table accompanied by five 60 -watt lamps that provided indirect illumination. At the start of the trial, each mouse was placed at the center of the box and received a $1 \mathrm{~h}$ session recorded over 5 min intervals. Locomotor activity was recorded using a 4x8 photobeam activity system by San Diego Instruments (San Diego, CA). The dependent variables are the total horizontal movements ( $\mathrm{x}, \mathrm{y}$ direction) and rearing movements (z direction) over a 60 min period.

\subsubsection{Accelerating rotarod (AR)}

The accelerating rotarod test was used to evaluate evoked locomotor performance and coordination as previously described [39] and was administered on day three post-sepsis. A textured plastic horizontal rod $(3 \mathrm{~cm}$ in diameter) was mounted $14.5 \mathrm{~cm}$ above a pressuresensitive base (Ugo Basile, Gemonio, Italy). For each trial, the mouse was placed on a moving rod that accelerates from $4 \mathrm{rpm}$ to $44 \mathrm{rpm}$ when the operator hits the start button. Acceleration is continued until the mouse fell onto the padded base or until 300 s elapsed. Latency to fall (s) onto the padded base was the dependent variable. All mice received 4 trials on the rotarod.

\subsubsection{Passive avoidance}

The passive avoidance test is used to assess non-spatial aversive learning and memory [40] and was administered days four and five post-sepsis. The test is performed using a twocompartment (illuminated and darkened) chamber connected by an automated door (Med Associates, VT). All animals undergo 3 trials (120 s acquisition, 15 min immediate retention, and $24 \mathrm{~h}$ retention). During the $120 \mathrm{~s}$ acquisition, each animal is placed in the illuminated compartment and receives a $0.3 \mathrm{~mA}$ shock when it enters the darkened compartment. For the 300 s retention trials, the animal is placed in the illuminated chamber; however, no shock is initiated upon entry into darkened compartment. The dependent variable is the latency to enter the darkened compartment (s). 


\subsubsection{Hot plate}

The hot plate test is used to assess nociception [41] and was administered on day five post-sepsis. Mice were individually placed on a preheated $55^{\circ} \mathrm{C}$ hot plate (IITC Life Science, $\mathrm{CA}$ ), inside an open-ended cylindrical Plexiglas tube with a diameter of $30 \mathrm{~cm}$. The latency (s) to respond to thermal stimuli and the total number of nociceptive behaviors (jumps as well as hind limb-lick, flick or flexion) exhibited over a 30 s time frame were measured. To prevent tissue damage, mice were removed from the hot plate after $30 \mathrm{~s}$ regardless of their response.

\subsubsection{Two-day radial arm water maze (2D-RAWM)}

The 2D-RAWM was used as previously described $[42,43]$ with minor modifications.

Briefly, a 6-arm maze is submerged in a pool of opaque water, and an invisible platform placed at the end of 1 arm below the surface of the water. Each mouse received 15 trials per day for two days and the start arm was changed on each trial, however, the goal arm remained the same for all animals. Using static visual cues, the mouse learned the position of the platform. The number of errors (incorrect arm entries) is counted across $1 \mathrm{~min}$ trials. If a mouse failed to locate the platform in $1 \mathrm{~min}$, the mouse was guided to the platform by the investigator and allowed to stay on the platform for $15 \mathrm{~s}$ before returning the mouse to a heated cage. The number of errors is averaged over two trials per block, resulting in five blocks (15 trials) per day per animal, and a total of 10 blocks (30 trials) for the two-day period. The latency to get to the platform (s) and the number of errors are the dependent variables measured. The 2D-RAWM was performed on days five (acquisition) and six (retention) post-sepsis. It is important to note that mice that participated in the hot plate test and the passive avoidance test did not participate in the 2D-RAWM and vice versa due to the stressful impact of both tests on the mice. To avoid any potential influence of aversive testing experience, a separate cohort of mice was used for $\mathrm{PA} / \mathrm{HP}$ testing than those that underwent 2D-RAWM. 


\subsubsection{Experimental design and statistical analysis}

All experiments were executed to enhance rigor and avoid experimenter bias according to ARRIVE guidelines [44]. Only male mice were used for all experiments because males are more susceptible to sepsis than females as previously published [45]. The use of male mice also allows for comparisons with a number of published studies which address similar outcomes $[2,16,46]$. Behavioral assays were conducted from the least aversive (i.e., open field) to most aversive (i.e., 2D-RAWM). The investigator was blinded to the treatment groups for all behavior tests and image analysis. Animals that died $24 \mathrm{~h}$ after a behavior test was conducted were excluded from the study. See Figure 1 for an experimental timeline and outline of behavioral assays performed. All statistical analyses were conducted in GraphPad Prism 8.1 (GraphPad Software, La Jolla, CA). Results are expressed as mean \pm SEM and $p$-values $\leq 0.05$ were considered significant. Survival was analyzed using a log-rank test. All other results were analyzed using a two-tailed unpaired Student's t-test, repeated two-way analysis of variance (ANOVA), or two-way analysis of variance (ANOVA) followed by Tukey's multiple comparisons post hoc test. Datasets that did not display a Gaussian distribution were subjected to a comparable non-parametric analysis as indicated in the text and figure legends. All $p$-values and $n$ values are indicated in the text and figure legends.

\subsection{Results}

\subsubsection{Survival analysis, sickness score, and average weight loss}

The objective of our first set of experiments was to characterize our sepsis model by investigating the detrimental effects of sepsis on mortality and sickness behavior. Septic mice had a significantly increased mortality rate compared to sham mice $\left({ }^{* * *} p=0.001\right.$, Log-rank $)$ (Fig. 2A). Thereafter, we examined sickness score and average weight loss using the modified murine sepsis score (MMSS). Our results showed a significantly increased sickness score in 
septic mice compared to sham mice $\left(F(1,26)=22.55,{ }^{* * * *} p<0.0001\right.$, repeated measures twoway ANOVA) (Fig. 2B). Furthermore, septic mice had a significantly higher average weight loss score compared to sham mice $\left(U(104,302)=26,{ }^{* * *} p=0.0005\right.$, Mann-Whitney test) (Fig. 2C). Due to the downward trend in the sickness score and an improvement in overall physical appearance of septic animals after day five, we examined blood serum levels of inflammatory cytokines interleukin 6 (IL-6) and monocyte chemoattractant protein-1 (MCP-1) chemokine on day seven in a terminal blood sample. Our results show that septic mice exhibit a persistent peripheral inflammatory phenotype denoted by the significant increase in the levels of inflammatory cytokines IL-6 $\left(\mathrm{U}(16,62)=1,{ }^{* *} p=0.005\right.$, Mann-Whitney test $)$ and MCP-1 (U (11, 55) $=1,{ }^{*} p=0.01$, Mann-Whitney test) (Fig. 2D).

2.4.2. TNAP's activity in brain microvascular endothelial cells is altered in early (24 h) and late sepsis (7 days)

Earlier studies have demonstrated a strong evidence for BBB dysfunction in early sepsis $[47,48]$. Hence, we examined whether loss of TNAP activity was also present as early as $24 \mathrm{~h}$ post-sepsis. Our results showed a significant loss of TNAP activity in the cerebral microvessels of septic mice compared to sham septic mice at $24 \mathrm{~h}$ post-sepsis (Supplementary Fig. 1). We next hypothesized that elevated levels of the serum pro-inflammatory cytokines, IL-6 and MCP1 (Fig. 2D), in late sepsis would support a sustained loss of TNAP enzyme activity in cerebral microvessels. To investigate this, we co-immunolabelled cerebral microvessels with the endothelial cell marker CD31 and subsequently quantified in vivo AP activity in the cerebral microvessels within the same tissue. It is important to note that TNAP is the only AP isoform found in microvessels of the brain and spinal cord $[49,50]$. Therefore, assessment of total AP activity is adequate to assess total TNAP activity in cerebral microvessels. Our in vivo results demonstrated a significant decrease in TNAP enzyme activity in the somatosensory cortex $(\mathrm{t}=$ 2.8, ${ }^{*} p=0.02$, unpaired t-test) (Fig. 3A, B) of septic mice. Parallel AP histology of the spinal 
cord also showed decreased TNAP enzyme activity in the spinal cords of septic mice

(Supplementary Fig. 2). Co-labeling of TNAP enzyme activity (purple) with cerebral

microvessels (CD31, brown) provides further support that the observed decrease in TNAP's enzyme activity is not the result of a loss of cerebral microvessels, and thus most likely demonstrates that TNAP enzyme activity is reduced in cortical cerebral microvessels during late sepsis (Fig. 3C). In contrast, we did not observe any significant differences in TNAP enzyme activity in the hippocampal CA1 or CA3 region of septic and sham mice on day seven (data not shown). Collectively, our findings reveal a sustained loss of cortical and spinal TNAP enzyme activity in late sepsis.

\subsubsection{TNAP is critical for maintaining cerebrovascular barrier integrity}

Past studies by Deracinois et al. revealed that inhibition of TNAP enzyme activity with a pan-AP inhibitor (levamisole) decreased barrier integrity in bovine capillary endothelial cells [20]. However, levamisole is a non-specific inhibitor of TNAP and is capable of decreasing the activity of other phosphatases $[31,51,52]$. Likewise, it is not clear whether TNAP inhibition during inflammation further exacerbates loss of barrier integrity. Therefore, we utilized a specific in vitro inhibitor of TNAP (TNAPi) to examine the effect of TNAP inhibition in hCMEC/D3 cells under basal conditions and during inflammation. Our results showed that treatment of hCMEC/D3 with TNAPi ( ${ }^{*} p=0.026$, Tukey's multiple comparison test, two-way ANOVA) or LPS $\left({ }^{*} p=0.01\right.$, Tukey's multiple comparison test, two-way ANOVA) significantly decreased barrier integrity to similar levels compared to DMSO treated cells. Furthermore, combination treatment of LPS and TNAPi significantly decreased barrier integrity when compared to TNAPi only $\left({ }^{* * *} p<0.0001\right.$, Tukey's multiple comparison test, two-way ANOVA), LPS only ${ }^{* * * *} p<0.0001$, Tukey's multiple comparison test, two-way ANOVA), and DMSO $\left({ }^{* * *} p<0.0001\right.$, Tukey's multiple comparison test, two-way ANOVA) treated hCMEC/D3 cells (Fig. 4A). As reported previously, the expression of the tight junction [17] protein claudin-5 is decreased at $24 \mathrm{~h}$ following either CLP 
or LPS injection $[53,54]$. We hypothesized that the sustained loss of TNAP enzyme activity in the somatosensory cortex (Fig. 2D) would be coupled to a persistent loss of brain microvascular integrity in late sepsis. To investigate this, we co-immunolabelled for the endothelial cell marker CD31 (brown) and claudin-5 (red) in the somatosensory cortex. Our results showed that claudin-5 expression around CD31-labelled microvasculature was decreased in the somatosensory cortex of septic mice compared to sham mice (Fig. 4B). However, we did not observe any differences in hippocampal claudin- 5 expression between septic and sham mice (data not shown). Since the TNAPI can only be used in vitro, we next used a highly specific in vivo TNAP inhibitor (SBI-425) to investigate whether further inhibition of TNAP enzyme activity exacerbates additional loss of cortical claudin- 5 in septic mice. Our results showed that SBI425 treated septic mice exhibited an increased loss of cortical claudin-5 compared to vehicletreated septic mice and naïve mice (Fig. 4C). Furthermore, co-immunolabelling for immunoglobulin G (IgG, brain permeability marker) and TNAP enzyme activity in the somatosensory cortex revealed increased vascular (cyan arrow) and perivascular lgG permeability (red arrow) in the brains of septic mice compared to sham mice (Fig. 4D). Our results also showed that septic cortical microvascular brain regions with moderate TNAP enzyme activity exhibited decreased IgG brain parenchymal infiltration (green arrow) compared to cortical microvascular brains regions devoid of TNAP enzyme activity (red arrow) (Fig. 4D). Together, our results demonstrate that modulation of TNAP enzyme activity during inflammation may be critical for barrier maintenance. Thus, both in vitro and in vivo findings support TNAP's critical role in maintaining endothelial barrier integrity in normal physiology and during inflammation.

\subsubsection{Dysfunction of the neuroimmune axis post-sepsis}

\subsubsection{Sustained peripheral myeloid immune cell trafficking into brain parenchyma of septic} mice 
To investigate whether alterations in TNAP activity within cerebral microvessels (Fig. 3A, B) are coupled to trafficking of peripheral immune cells into the brain parenchyma at day seven post-sepsis, we next characterized the percentage of myeloid cell populations in the brains of septic and sham mice using flow cytometry. Myeloid cells are gated using the strategy shown in Supplementary Figure 3. FCS express analyses of inflammatory myeloid cell populations showed that neutrophils (CD45 hi, CD11 b+ Ly6C $^{-}$, Ly6G $\left.^{+}\right)$(Fig. 5A, B) and monocytes (CD45 hi, CD11C ${ }^{+}$, Ly6C $^{+}$, Ly6G $\left.^{-}\right)$(Fig. 5C, D) are significantly increased $(U(15,51)=$ $0,{ }^{* *} p=0.004$, Mann-Whitney test) in the brains of septic compared to sham mice. Myeloid derived suppressors cells (MDSCs) i.e., polymorphonuclear-MDSCs/PMN-MDCs (CD45 hi, CD11b+, Ly6C ${ }^{10}$, Ly6G $\left.^{+}\right)$(Fig. 5E, F; U $(15,51)=0,{ }^{* *} p=0.004$, Mann-Whitney test) and monocytic-MDSCs/M-MDSCs (CD45 hi, CD11b+, Ly6C ${ }^{\text {hi }}$, Ly6G') (Fig. 5E, G; U $(15,51)=0,{ }^{* *} p=$ 0.004 , Mann-Whitney test) were also significantly increased in the brains of septic compared to sham mice.

\subsubsection{Sustained peripheral T-lymphoid immune cell trafficking into brain parenchyma of septic} mice

Next, we explored T-lymphoid immune cell trafficking post-sepsis using the gating strategy for CD45 hi live cells shown in Supplementary Figure 4. FCS express analyses of $\mathrm{CD} 8+\left(\mathrm{CD} 45^{\mathrm{hi}}, \mathrm{CD}^{+}, \mathrm{CD}^{+}\right)\left(\right.$Fig. 6A, B $; \cup(15,51)=0,{ }^{* *} p=0.004$, Mann-Whitney test $)$ and CD4+ T-cells $\left(C D 45^{\text {hi }}, \mathrm{CD}^{+}, \mathrm{CD}^{+}\right)$(Fig. 6C, D; $\mathrm{t}=2.3,{ }^{*} p=0.046$, unpaired t-test) revealed increased populations of these cells in the brains of septic compared to sham mice. We also assessed whether T-regulatory (Tregs) cells from the T-lymphoid lineage were similarly increased in the brains of septic mice. To do this, CD4+ T-cells were gated further for Foxp3 and CD25 markers. Our results showed that Treg cells $\left(\mathrm{CD} 4^{+}, \mathrm{CD} 25^{+}\right.$, Foxp3 $\left.{ }^{+}\right)$(Fig. 6E, F) are significantly increased $\left(\mathrm{t}=4.7,{ }^{* *} p=0.0011\right.$, unpaired $\mathrm{t}$-test $)$ in the brains of septic mice 
compared to sham mice. Together both findings from Figures 5A-G and 6A-F demonstrate a persistent dysfunction in the neuroimmune axis in septic mice.

\subsubsection{Regional analyses of brain residential microglia populations in late sepsis}

Our next step was to determine the effects of sepsis associated brain microvascular dysfunction on residential microglia cell populations. For our analyses, we performed immunohistochemistry and assessed microglia in three different brain regions (i.e., somatosensory cortex, CA1, and CA3) for the microglia/macrophage ionized calcium binding adaptor molecule 1 (Iba-1). Iba-1 immunoreactive cells were significantly increased in the somatosensory cortex $\left(U(15,51)=0,{ }^{* *} p=0.004\right.$, Mann-Whitney test $)$, and CA1 $\left(\mathrm{t}=3.5,{ }^{* *} p=\right.$ 0.007 , unpaired t-test $)$ and CA3 $\left(t=3.9,{ }^{* *} p=0.004\right.$, unpaired $t$-test $)$ hippocampal regions of the brain (Fig. 7). Further analysis for lba-1 positive cells in the spinal cords of septic mice were increased compared to sham mice (Supplementary Figure 5A). To confirm whether the stained Iba-1 positive cells were microglia and not macrophages, we stained for microglia using a specific marker, TMEM119, which is not found on macrophages [55]. Contrary to the observed increase in Iba-1 immunoreactive cells shown in Figures 7 A-F, there were no significant differences in TMEM119 immunoreactive cells in the somatosensory cortex $(\mathrm{t}=0.22, p=0.13$, unpaired $\mathrm{t}$-test $)$, and the CA1 $(\mathrm{t}=0.07, p=0.94$, unpaired $\mathrm{t}$-test $)$ and $\mathrm{CA} 3(\mathrm{t}=0.42, p=0.68$, unpaired t-test) hippocampal regions (Supplementary Table 1). This suggests that these cells are infiltrating monocytes rather than resident microglia.

\subsubsection{Sustained astrogliosis in the somatosensory cortex of septic mice}

Given the alterations in brain microglial/monocyte activation, we also assessed changes in astrocyte proliferation and morphology post-sepsis. We performed glial fibrillary acidic protein (GFAP) immunolabeling and quantified the proliferation of reactive astrocytes in the somatosensory cortex, in hippocampal CA1 and CA3 and in the spinal cord. Our results 
demonstrate a significant increase in astrocyte proliferation in the somatosensory cortex $(\mathrm{t}=$ $3.5,{ }^{* *} p=0.006$, unpaired t-test) of septic compared to sham mice (Fig. 8A, B). However, we did not observe any significant differences in astrocyte proliferation in the CA1 $(\mathrm{t}=0.5, p=0.61$, unpaired t-test $)$ and CA3 $(\mathrm{t}=0.60, p=0.56$, unpaired $\mathrm{t}$-test $)$ hippocampal regions of the brain (Fig. 8C-F). Parallel to the results observed in the somatosensory cortex of the brain, we observed an increase in proliferative astrocytes in the spinal cords of septic mice compared to sham mice (Supplementary Figure 5B). These results demonstrate that the somatosensory cortex and the spinal cord exhibit sustained astrogliosis post-sepsis.

\subsubsection{Septic mice displayed an impairment in spontaneous locomotion and exhibited a novel anti-nociception phenotype}

Septic and critically ill patients often experience long-term sensorimotor impairment [12, $13,56]$. Therefore, we implemented a series of behavioral assays on days two (open field testing, OFT) and three (rotarod) to assess motor dysfunction and on day five (hot plate) to assess sensory dysfunction in septic mice. The OFT was used to evaluate spontaneous motor dysfunction while the rotarod was utilized to assess evoked motor dysfunction. Our results showed that spontaneous locomotion in the horizontal $\left(F(1,24)=20.51,{ }^{* \star *} p<0.0001\right.$, repeated measures two-way ANOVA $)$ and vertical axes $\left(F(1,24)=48.4,{ }^{* * *} p<0.0001\right.$, repeated measures two-way ANOVA) were significantly decreased in septic compared to sham mice on day two over a 60 min trial (Fig. 9A, B). However, we did not observe any differences in evoked locomotion on the rotarod $(F(1,13)=1.6, p=0.23$, repeated measures two-way ANOVA) between septic and sham mice over four $300 \mathrm{~s}$ trials (Fig. 9C). Next, we utilized the hot plate test to assess sensory dysfunction post-sepsis. Our results show that septic mice have an increased latency to respond to thermal stimuli $\left(t=2.8,{ }^{* *} p=0.01\right.$, unpaired $t$-test $)$ and exhibit a decreased total number of nociceptive behaviors (hind limb-lick, flick, and jump) $(\mathrm{t}=$ 2.9, ${ }^{* *} p=0.0096$, unpaired t-test) compared to sham mice (Fig. 9D, E). From these findings, we 
report, for the first time, a discrepancy between two measurements of locomotion, OFT and rotarod, in septic mice, and reveal a novel anti-nociceptive phenotype exhibited by septic mice.

\subsubsection{Learning and memory is preserved seven days post-sepsis}

To determine whether learning and memory deficits are evident during late sepsis, we used the two-day radial arm water maze (2D-RAWM) to assess for spatial learning and memory on days five and six, and the passive avoidance test to assess non-spatial learning and memory on days four and five. Septic mice showed no difference in the number of errors $(F(1,20)=$ $0.76, p=0.39$, repeated two-way ANOVA) or the latency to find the hidden platform $(F(1,20)=$ $0.87, p=0.36$, repeated two-way ANOVA) on day two of the retention trial compared to sham mice (Fig. 10A, B). Assessment of non-spatial learning and memory with the use of the passive avoidance test showed no difference in latency to enter the darkened compartment $(F(1,12)=$ $0.09, p=0.77$, repeated two-way ANOVA) between septic and sham mice at the 15 min and 24 $\mathrm{h}$ retention trials (Fig. 10C). Due to the absence of learning and memory impairments at these timepoints, we assessed neuronal loss via NeuN immunolabeling in hippocampal regions CA1 and CA3. No apparent differences in neuronal loss were observed in these regions between sham and septic mice (Supplementary Figure 6). To confirm that other brain regions involved in memory consolidation were not affected by sepsis, we performed immunostaining for choline acetyltransferase [36] in cholinergic neurons of the basal forebrain. Similarly, to results observed in the hippocampus, there were no significant differences in the number of ChAT positive neurons in the basal forebrain of septic mice compared to sham mice (Supplementary Table 2). Taken together, the absence of neuronal loss in the hippocampus and cholinergic neurons of the basal forebrain in septic mice further substantiated the results derived from the 2D-RAWM and passive avoidance behavioral tests (Fig. 10A-C).

\subsection{Discussion}


Brain function in humans is significantly impaired in both early and late sepsis and is an independent predictor of mortality [14]. An emerging hypothesis suggests that the pronounced hyper-inflammatory phase seen in early sepsis disrupts brain microvascular function and barrier integrity $[57,58]$. The subsequent infiltration of peripheral immune cells and systemic proinflammatory molecules into the brain parenchyma that accompanies changes in brain microvascular dysfunction may increase the likelihood of significant short-term and long-term deficits in neurocognitive function [16, 59]. Therefore, a better understanding of brain microvascular mechanisms that maintain barrier integrity may foster the development of therapeutics to mitigate the neurological impairments seen in sepsis survivors. The results from this study support a mechanism through which loss of TNAP activity is linked to brain microvascular dysfunction. Our results demonstrate that TNAP is critically important for maintenance of brain microvascular integrity in model of experimental sepsis and that perhaps modulation of TNAP's enzymatic activity may foster the development of new therapies that treat long-term sepsis associated cognitive impairment.

We employed the cecal ligation and puncture model of experimental sepsis to identify neuroimmune mechanisms and behavioral deficits that accompanied brain microvascular dysfunction following a loss of TNAP enzyme activity in cerebral microvessels. Results from our sepsis model closely align with previously reported findings in similar models of sepsis [2, 60]. However, a unique feature of our study involves our focus on the relationship between suppressed TNAP enzyme activity to brain microvascular dysfunction, leukocyte trafficking, and behavioral deficits in late sepsis. Very little is known about TNAP-associated functions and signaling mechanisms outside of its well-characterized role in bone and mineral metabolism [61]. The physiological importance of suppressed TNAP activity in brain endothelium was suggested by an earlier in vitro study which showed that inhibition of TNAP with a nonspecific pan-AP inhibitor (levamisole) worsened brain endothelial cell barrier integrity [20]. However, the experiments in this study established a role for TNAP in basal endothelial cell permeability but 
did not address the effects of TNAP inhibition during inflammation or injury. Moreover, levamisole is a nonspecific AP inhibitor that induces neurological deficits $[31,62]$. Thus, we carried out a series of in vitro and in vivo studies with highly specific TNAP inhibitors to further investigate a putative role for TNAP in brain endothelium. Our findings in the current study expand on previously published data showing that TNAP's enzymatic activity is decreased in septic mice $24 \mathrm{~h}$ post-sepsis [5], i.e., early sepsis, and we demonstrate that suppression of TNAP's enzymatic activity extends into late sepsis.

Results from the current study also reveal a mechanistic role for TNAP as an important regulator of barrier function during inflammation. First, our in vitro results in hCMEC/D3 cells demonstrated that inhibition of TNAP activity significantly suppressed barrier function in brain endothelium over time. More importantly, TNAP inhibition combined with LPS treatment synergistically worsened barrier function compared to LPS or TNAPi alone. Second, in vivo results showed that a decrease in claudin- 5 coupled with increased extravasation IgG in the somatosensory cortex suggests that inhibition of TNAP is coupled to cerebral microvascular dysfunction. Furthermore, in vivo inhibition of TNAP on cerebral microvessels of septic mice with SBI-425 decreased claudin 5 immunolabeling in the somatosensory cortex. However, a limitation of our SBI-425 studies is that because TNAP is expressed in numerous brain cell types [63], and it is likely that SBI-425 crosses the BBB in sepsis. Therefore, we cannot discern whether the effects SBI-425 on brain endothelial cell cells are direct or indirect due to crosstalk with other brain resident cells that express TNAP, e.g., neurons, astrocytes. Of note, there was no detectable loss of hippocampal TNAP enzyme activity or claudin- 5 in this model. These findings suggest that specific brain regions are more susceptible to sepsis-induced brain dysfunction than others in late sepsis. Collectively, our experiments demonstrate that TNAP modulation may be critical in attenuating the brain microvascular dysfunction described in many published preclinical and clinical sepsis studies [57]. 
Our experiments also addressed whether alterations in brain myeloid and lymphoid cell populations paralleled the loss of brain microvascular integrity. While alterations in peripheral myeloid and T-lymphoid cell populations have been described in numerous murine sepsis studies [64, 65], few studies have addressed changes in brain immune cells populations and trafficking peripheral leukocyte populations in late sepsis. Our results are consistent with findings from Singer et al. who also showed that monocytes and neutrophils persist up to day 14 in septic rodent brains [15]. Importantly, our findings showed that elevated numbers of leukocytes are present in the brain parenchyma post-sepsis in spite of a downward trend in sickness score, physical appearance of animal, and cessation of additional mortality past day five. Additionally, we also uncovered two novel findings that support a role for brain immunosuppression in late sepsis. It has been demonstrated that sepsis-associated immunosuppression in peripheral tissues contributes to poor recovery and long-term morbidity post-sepsis $[66,67]$, but a role of brain immunosuppression in sepsis is less clear. There are no reports, to our knowledge, that have investigated alterations in brain T-regulatory cell or MDSC populations in sepsis, our observation that brain $\mathrm{CD} 4^{+} / \mathrm{CD} 25^{+} / \mathrm{Foxp}^{+} \mathrm{T}$-regulatory cells (Tregs) were elevated in septic mice suggests that dysregulation of peripheral Tregs may play a role in generating the immunosuppressive phenotype associated with late sepsis [68-70]; however, further studies are needed to substantiate this finding. While the role of MDSCs in neuroinflammation is not well understood, a recent study in a mouse model of traumatic brain injury showed that circulating PMN-MDSCs and M-MDSCs entered the brain shortly after injury to suppress neuroinflammation; however, PMN-MDSCs declined thereafter while M-MDSCs remained elevated in the brain [71]. In contrast, the results from our study suggest that both PMN-MDSCs and M-MDSCs remain elevated in late sepsis. While MDSCs have been shown to express an immunosuppressive phenotype, we did not perform a functional analysis of MDSC populations and cannot determine whether this population contributes to sepsis-associated immunosuppression. 
Due to a persistent presence of brain microvascular dysfunction post-sepsis, we also investigated the impact of these changes on two key glial cell populations: microglia and astrocytes. Prior studies that have utilized Iba-1 and CD11b as markers to assess alterations in microglia populations have shown that microglia numbers are increased in the cortex and hippocampus of septic mice [15, 72]. In agreement with prior reports, we found that Iba-1 positive cell populations were increased in cortex and hippocampus. It is important to note that the Iba-1 and CD11b markers label microglia and/or infiltrating monocytes [72, 73]. Further immunostaining with TMEM119, a marker for residential microglia, did not reveal any differences between microglial populations in sham and septic mice. The observed differences reflect the heterogeneity of the brain microglial/monocyte populations and emphasize their importance in the pathophysiology of sepsis $[74,75]$. These findings further suggest that the resultant increase in lba-1 positive cells may be attributed to infiltrating monocyte populations that differentiate into macrophages expressing Iba-1.

Clinical studies have shown that elevated serum GFAP concentrations are associated with a poor prognosis and quality of life post-sepsis [76]. Similarly, preclinical studies have shown that GFAP-mediated astrogliosis is associated with brain microvascular dysfunction in many neuroinflammatory conditions [3]. Therefore, we speculated that the presence of brain microvascular dysfunction in late sepsis would support sustained astrogliosis in the somatosensory cortex and spinal cord but not in the hippocampus because there were no differences in hippocampal TNAP enzyme activity between sham and septic mice. Our results confirmed prominent astrogliosis in the somatosensory cortex and spinal cord. Taken together, our results demonstrate that brain microvascular dysfunction is also coupled to sustained microgliosis and astrogliosis in late sepsis.

Since septic survivors are burdened with neurological impairments [12], we investigated whether the neuroinflammatory findings in our sepsis model paralleled sensorimotor, nociceptive, and learning and memory impairments. Importantly, we identified several novel 
behavioral outcomes that are supported by findings in human sepsis survivors. We speculate that the significant decrease in spontaneous locomotion we observed was due to a lack of motivation to explore a novel environment post-sepsis; however, when septic mice were forced to engage in motor activity using the rotarod test, this motor impairment disappeared. These differences may be due to an effect of sepsis on motivation. Motivation is an affective behavior that is difficult to quantify in mice [77], and sepsis-induced changes in motivation have been observed following lipopolysaccharide injection [78]. Recent studies have also reported similar neuropsychiatric disturbances in motivation in sepsis survivors $[79,80]$. We also assessed deficits in thermal sensory dysfunction using the hot plate test since altered pain perception has previously been reported in sepsis patients [81]. Increased sensitivity to deep pain but not cutaneous pain has been reported in studies of human experimental endotoxemia [82]. Our results uncovered a novel anti-nociceptive phenotype in septic mice. The mechanism through which the observed anti-nociceptive phenotype is unclear, but we speculate that this phenotype may result from loss of TNAP enzyme activity observed in the somatosensory cortex and spinal cord, as these regions are part of critical neural circuits involved pain perception and processing biobehavioral pathways [83].

In contrast to previous studies, we did not observe any alterations in either spatial or non-spatial learning and memory tests between septic and sham mice. The absence of neuronal loss in the basal forebrain or the hippocampus supports these behavioral findings. It is highly likely that the seven-day post-sepsis time frame was too early to detect cognitive impairment, as other studies have shown that learning and memory are significantly altered at one-month post-sepsis in mice and may be mediated via infiltration of CCR2 ${ }^{+}$monocytes and/or increased levels of high mobility group box 1 protein (HMGBI) secreted by inflammatory cells $[16,84]$. Conceivably, activation of TNAP enzyme activity may limit the infiltration of CCR2+ monocytes and HMGBI into the brain and prevent the cognitive impairment seen one-month post-sepsis. It is also plausible that TNAP activity may be suppressed in other brain regions, 
e.g., hippocampus, at later time points such as one-month post-sepsis when learning and memory deficits typically become more apparent. Thus, a limitation of our behavioral studies is that they spanned from day 2 (early sepsis) up to day six (late sepsis).

In summary, this study establishes that diminished TNAP enzyme activity in cerebral microvessels is a consequence of the brain's sustained response to systemic inflammation and infection, i.e., sepsis. We propose a model in which TNAP functions as part of a dynamic complex of barrier integrity proteins on the luminal and abluminal surfaces of cerebral microvessels. Inhibition of TNAP activity disrupts this complex, thereby resulting in the loss of tight junction proteins, e.g., claudin-5, elevated extravasation of circulating proteins, e g. IgG, and increased leukocyte trafficking into the brain parenchyma. In turn, these events exacerbate neuroinflammation and behavioral deficits in sepsis survivors. A better understanding of these mechanisms will support the development of targeted therapies to mitigate chronic neurological impairments in sepsis survivors. It is highly likely that this novel regulatory mechanism extends beyond sepsis and encompasses a common mechanism of cerebral microvascular dysfunction in both acute and chronic systemic inflammatory disorders that cause neurological impairment.

\section{Author Contributions}

D.C.N, D.D, and C.M.B designed the studies. D.C.N. and A.L.B. performed CLP surgeries and monitored animals after sepsis procedure. D.C.N., C.A.G., and E.B.E-C. performed behavioral tests and analyzed behavioral data. D.C.N. and S.A.B. performed immunohistochemistry and image analysis. D.C.N., S.C., W.W., and S.J. performed and analyzed flow cytometric data. D.C.N., D.D., C.M.B. wrote the manuscript. JL.M. and A.B.P. provided the SBI-425 reagent. All authors read and revised the final manuscript.

\section{Acknowledgements}


The authors gratefully thank Jessica Povroznik, M.S. for her technical support and assistance with rodent behavioral training. We acknowledge the technical support from Dr. Kathleen Brundage in the West Virginia University Flow Cytometry and Single Cell Core Facility, which is supported by the National Institutes of Health $(\mathrm{NIH})$ equipment grant number S10 OD016165 and the Institutional Development Awards (IDeA) from the National General Medical Sciences of the National Institutes of Health under grant numbers P30 GM103488 (Cancer CoBRE) and P20 GM103434 (INBRE). Funding for this work was supported by the NIH T32 AG052375 (D.C.N, A.L.B), K01 NS081014 (C.M.B), West Virginia Clinical and Translational Science Institute (U54 GM104942), and the West Virginia University Stroke CoBRE (P20 GM109098). 


\section{Figures}

\section{Figure 1}

\section{Experimental Timeline}

\section{Cohort 1}

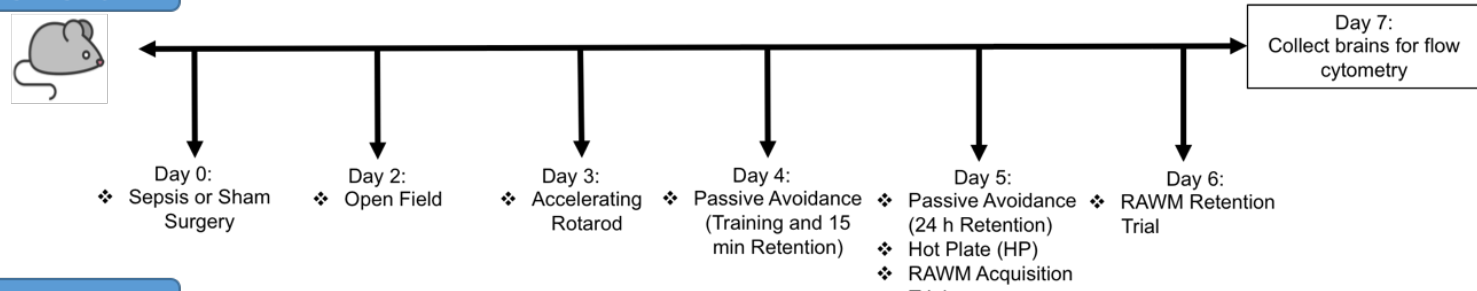

\section{Cohort 2}

$\underbrace{2}$

* Sepsis or Sham

Surgery

* $\quad$ Open Field

\section{Surgery}

\section{Cohort 3}

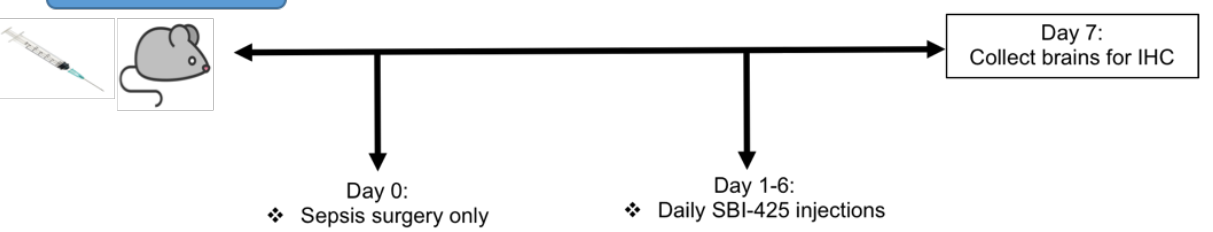


Figure 2

A

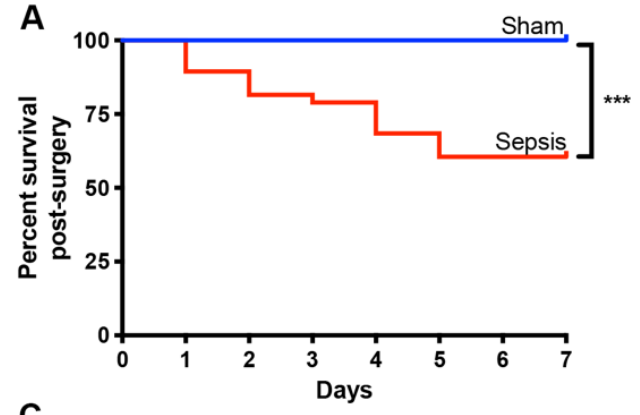

B
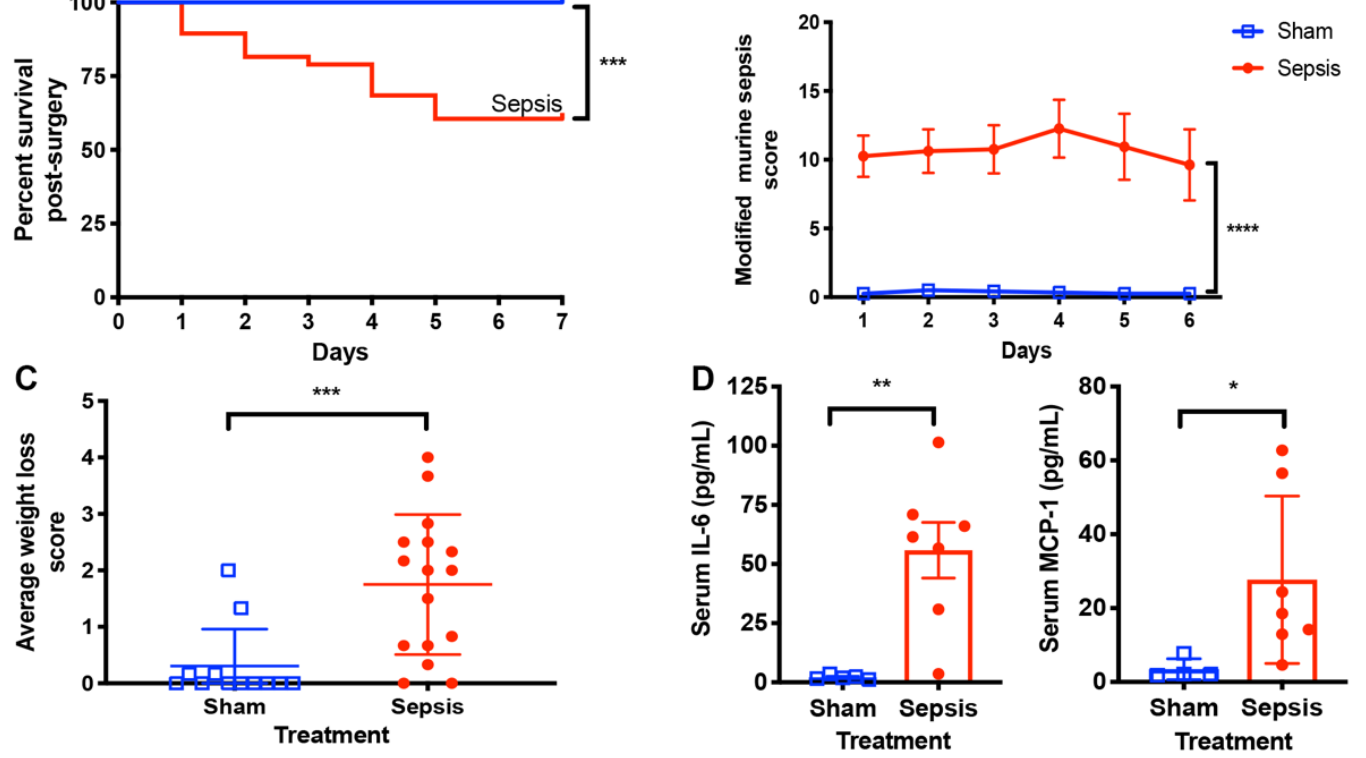
Figure 3

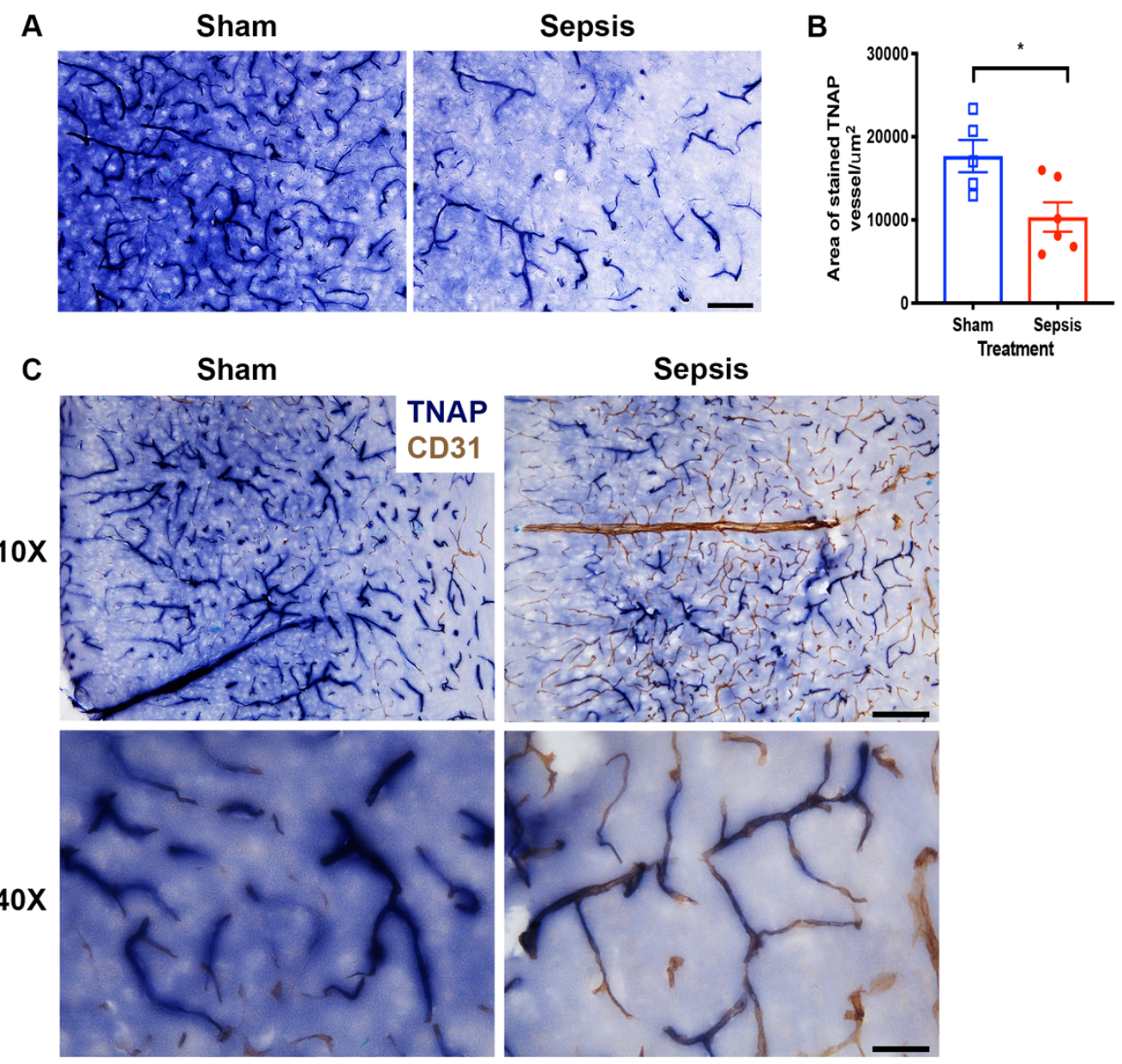


Figure 4

A

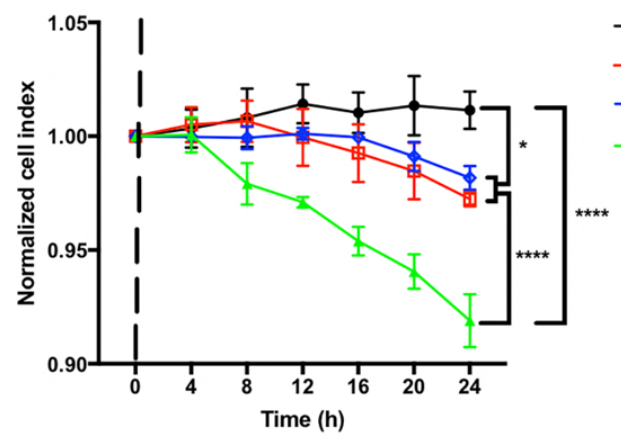

C

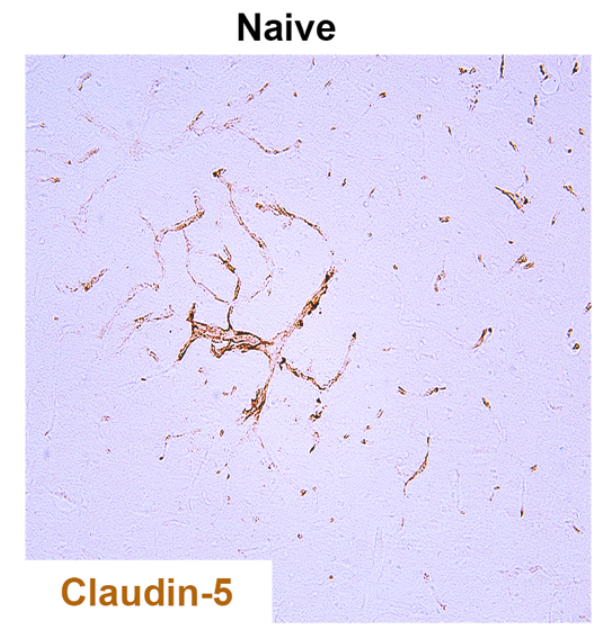

B
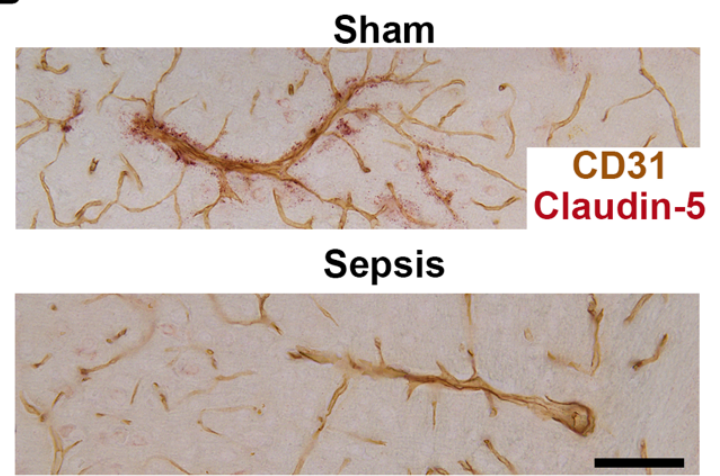

Sepsis + Veh 1

Sepsis + Veh 2

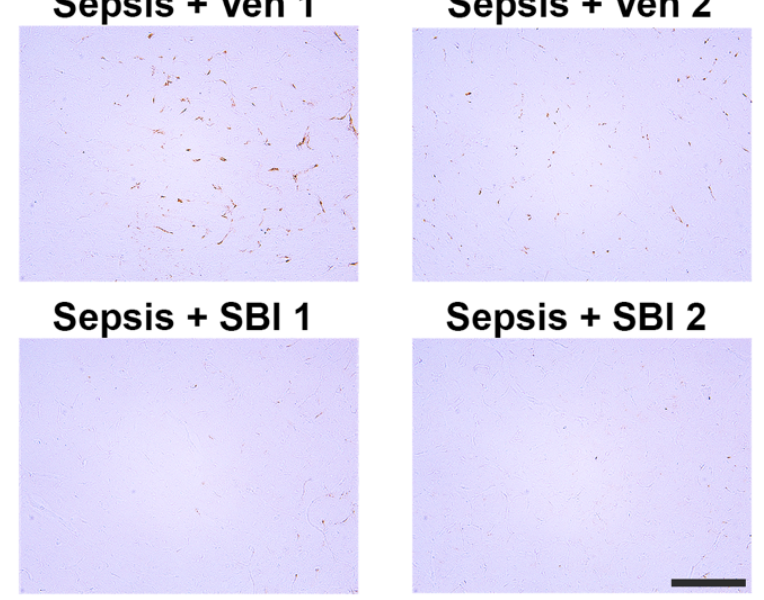

D

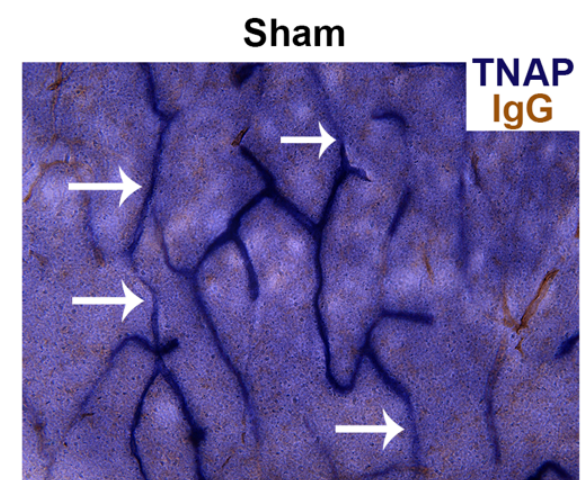

Sham
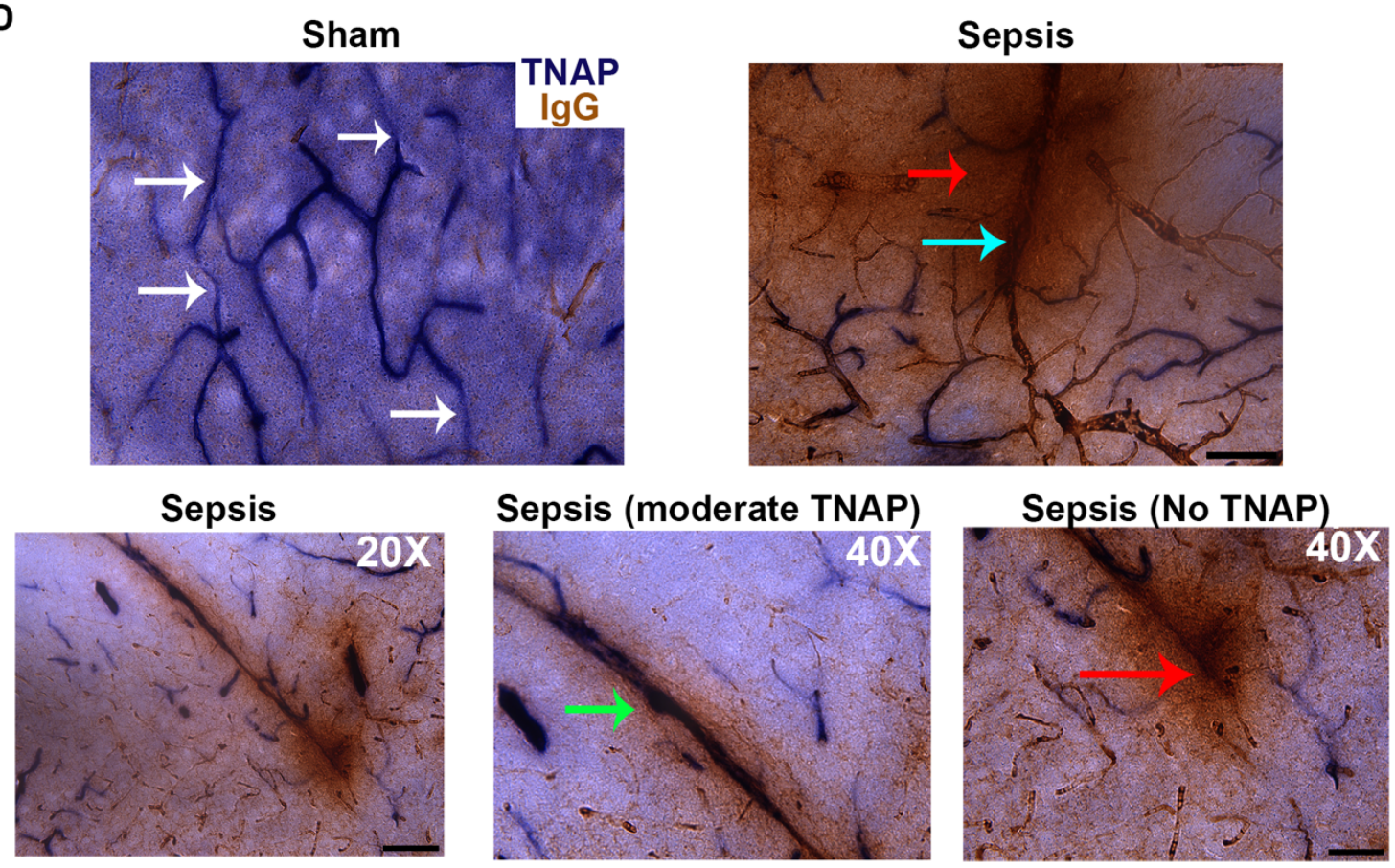
Figure 5

A

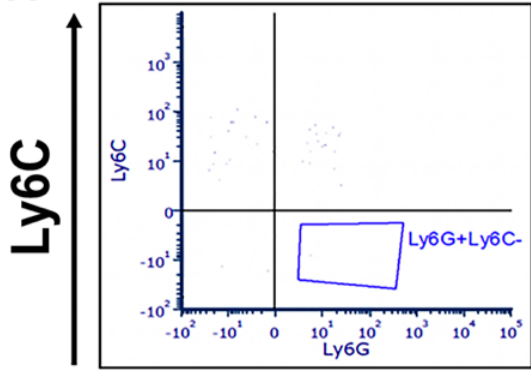

Sham

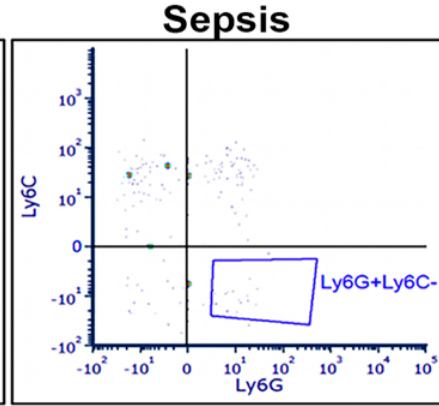

Ly6G

C

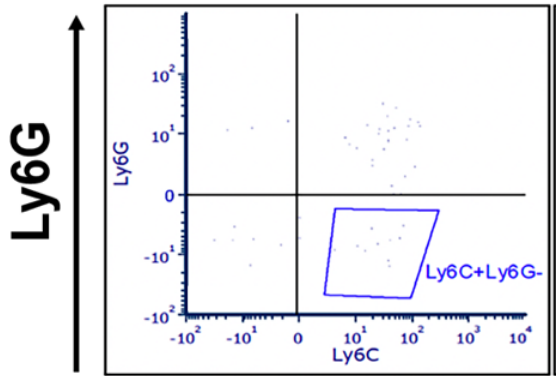

Ly6C

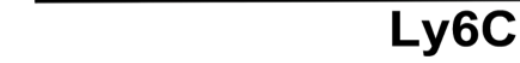

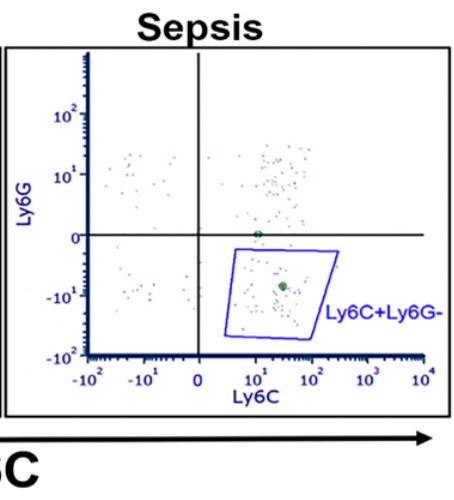
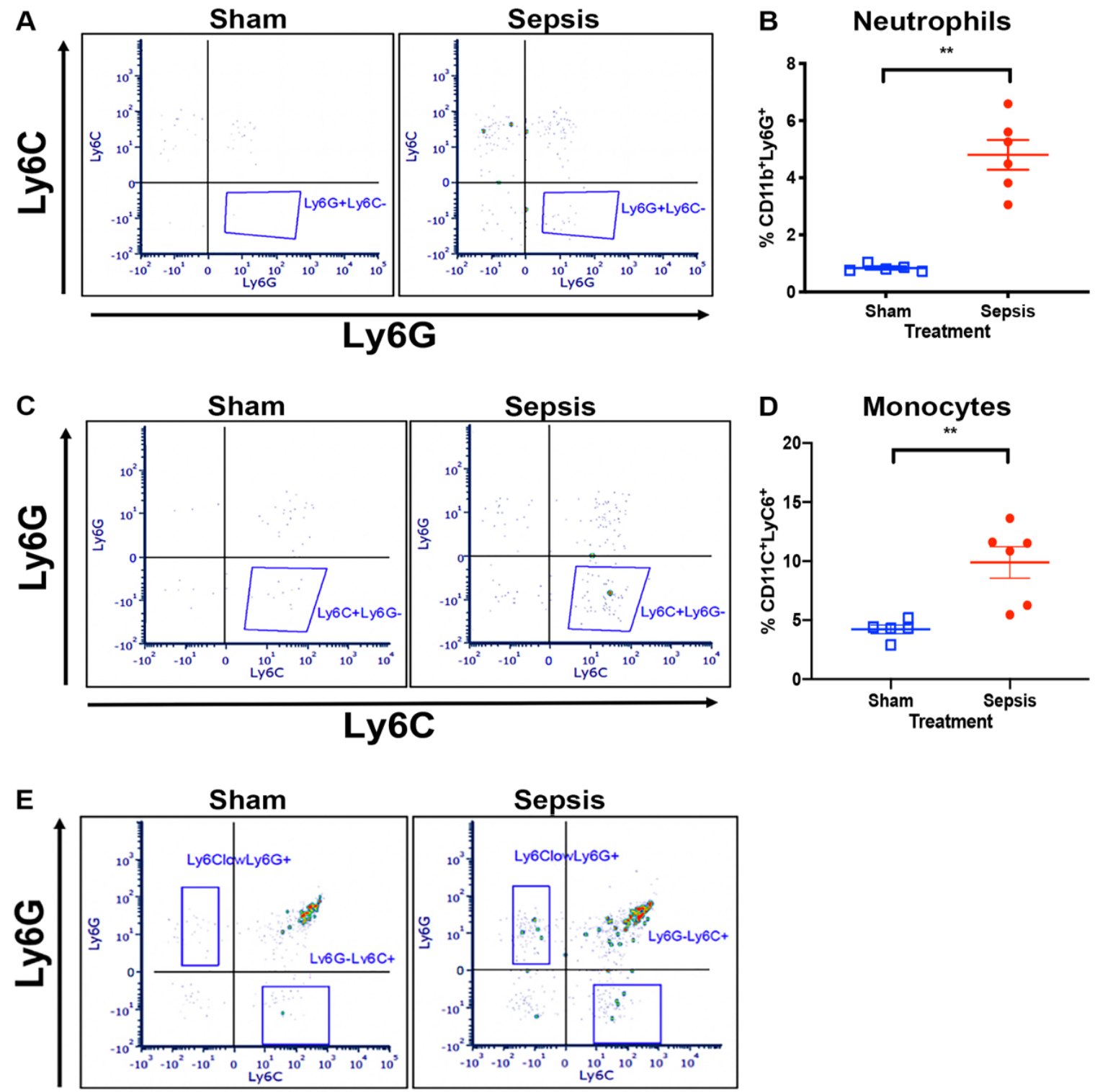

Sepsis

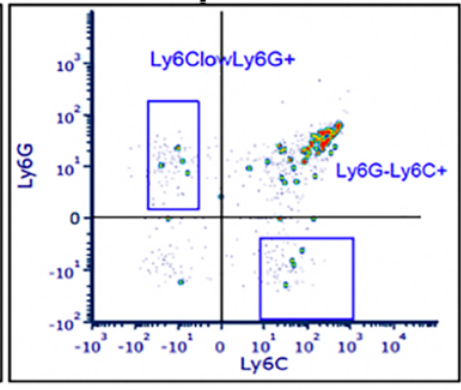

Ly6C

F PMN-MDSCs

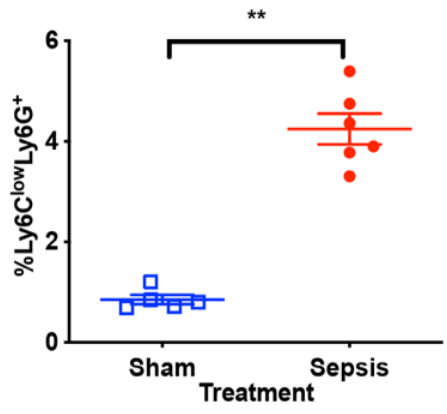

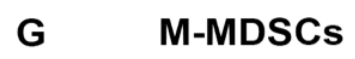

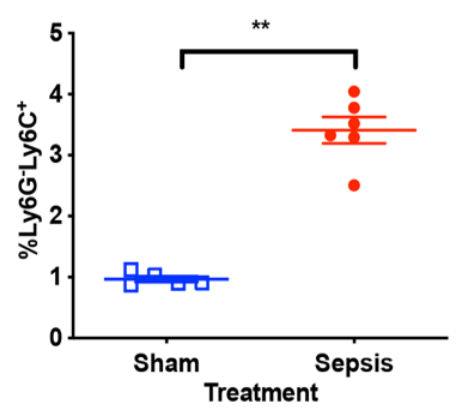


Figure 6
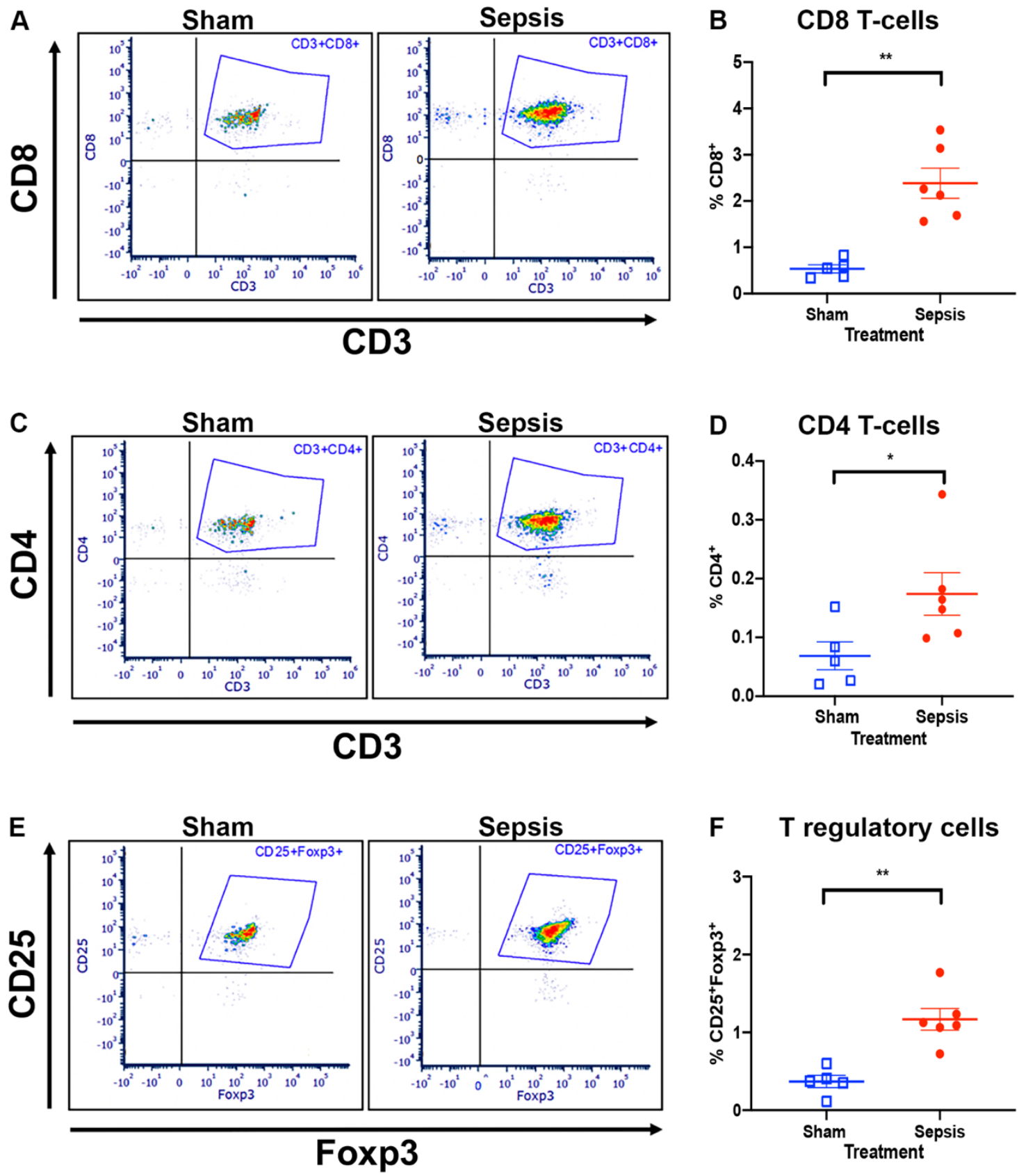
Figure 7
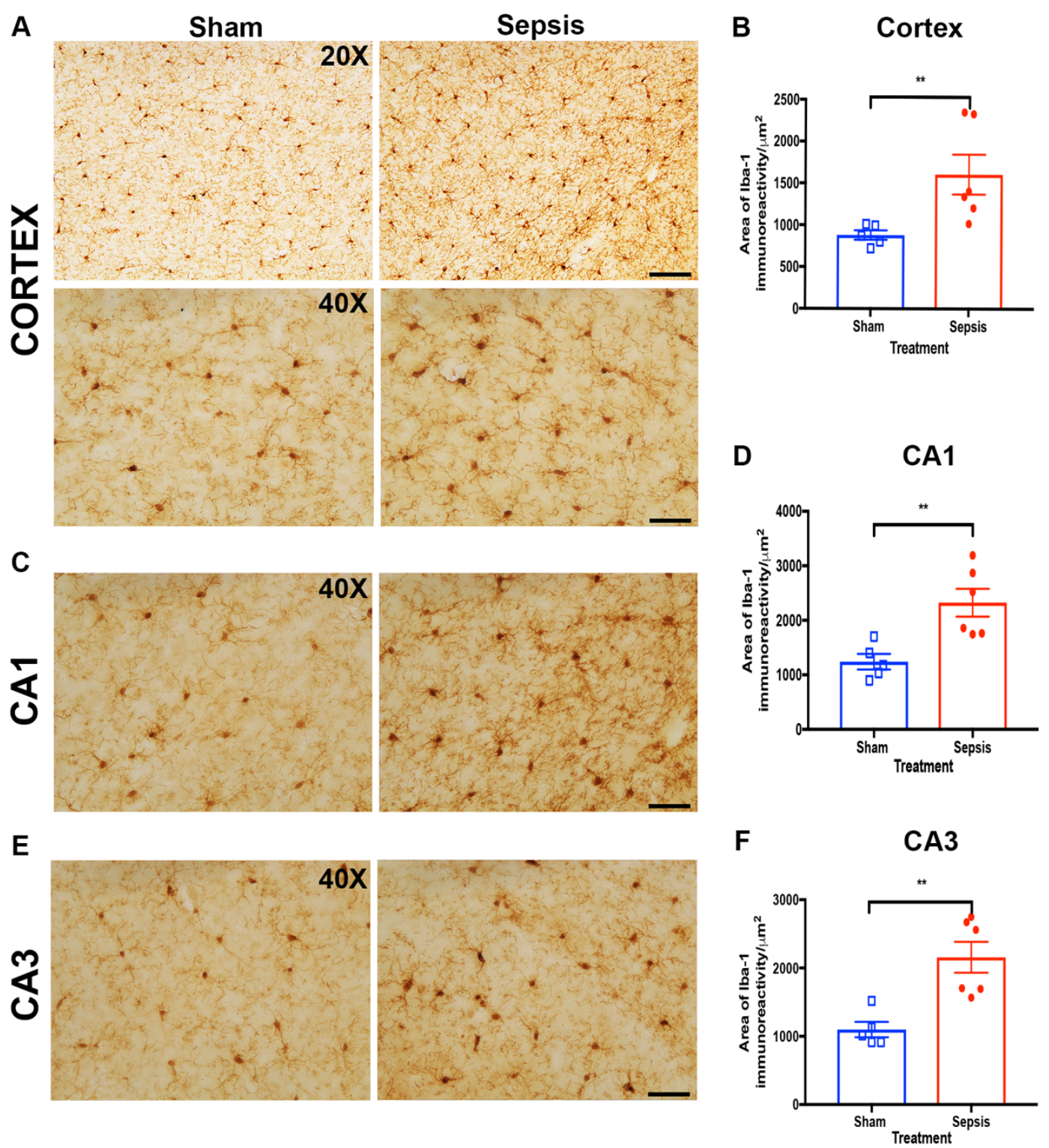
Figure 8

A

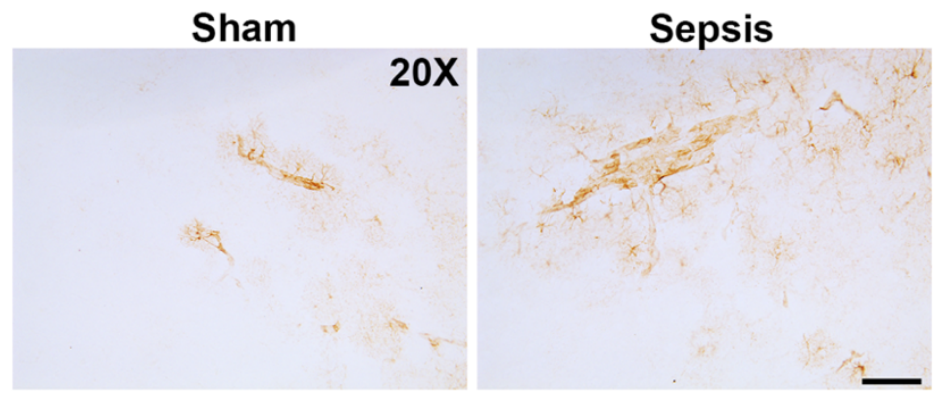

C

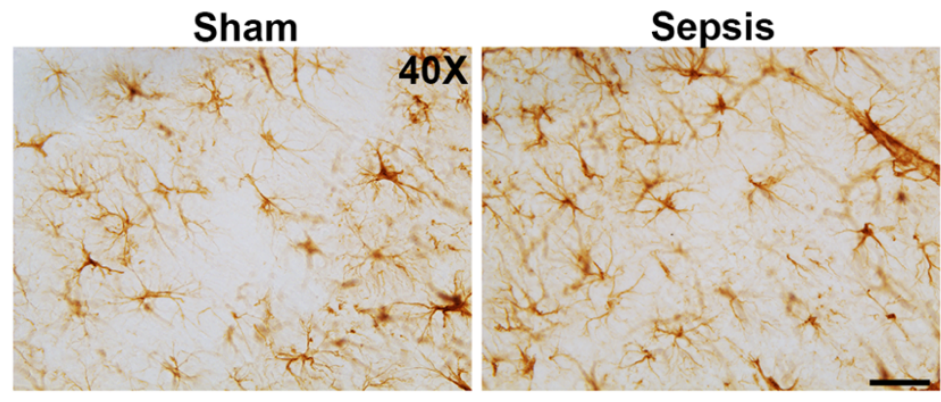

E

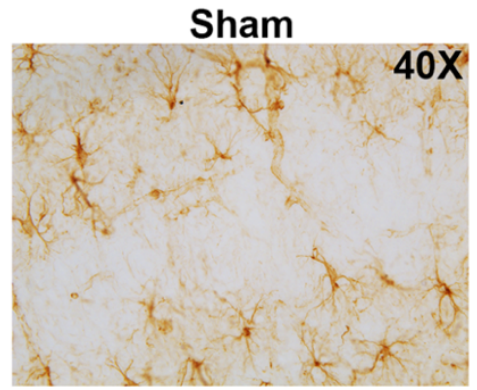

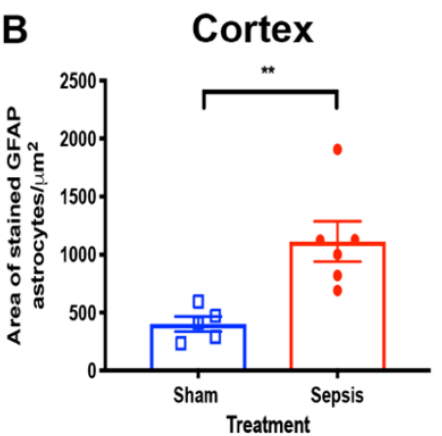

D CA1

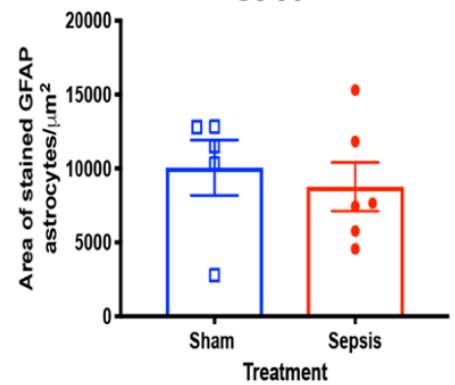

F

CA3

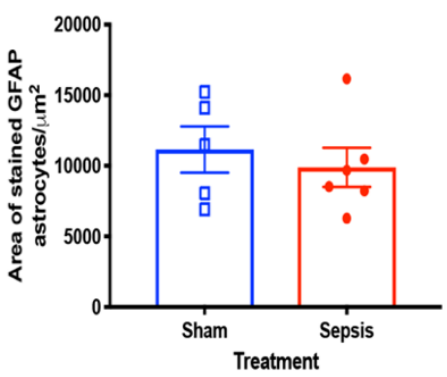




\section{Figure 9}

A

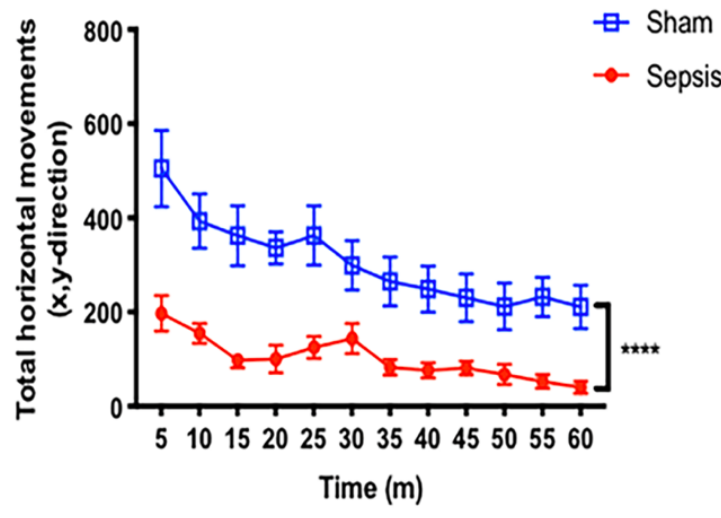

B

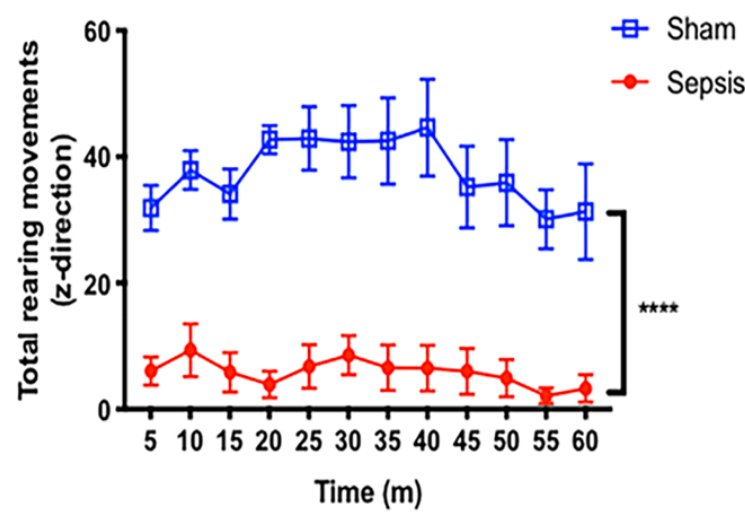

C

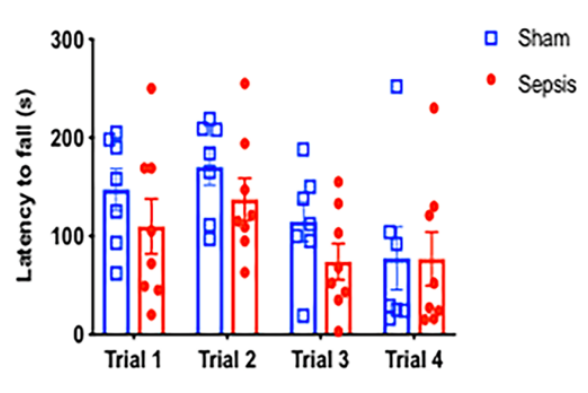

D

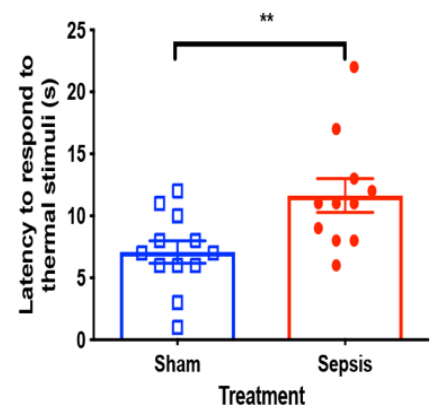

E

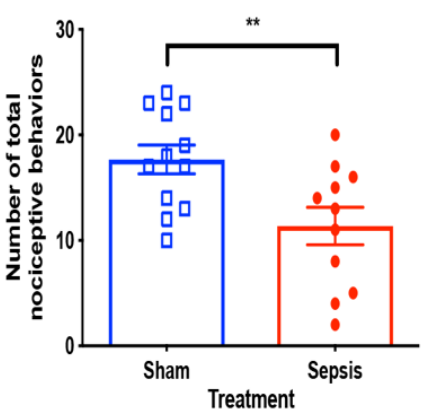


Figure 10
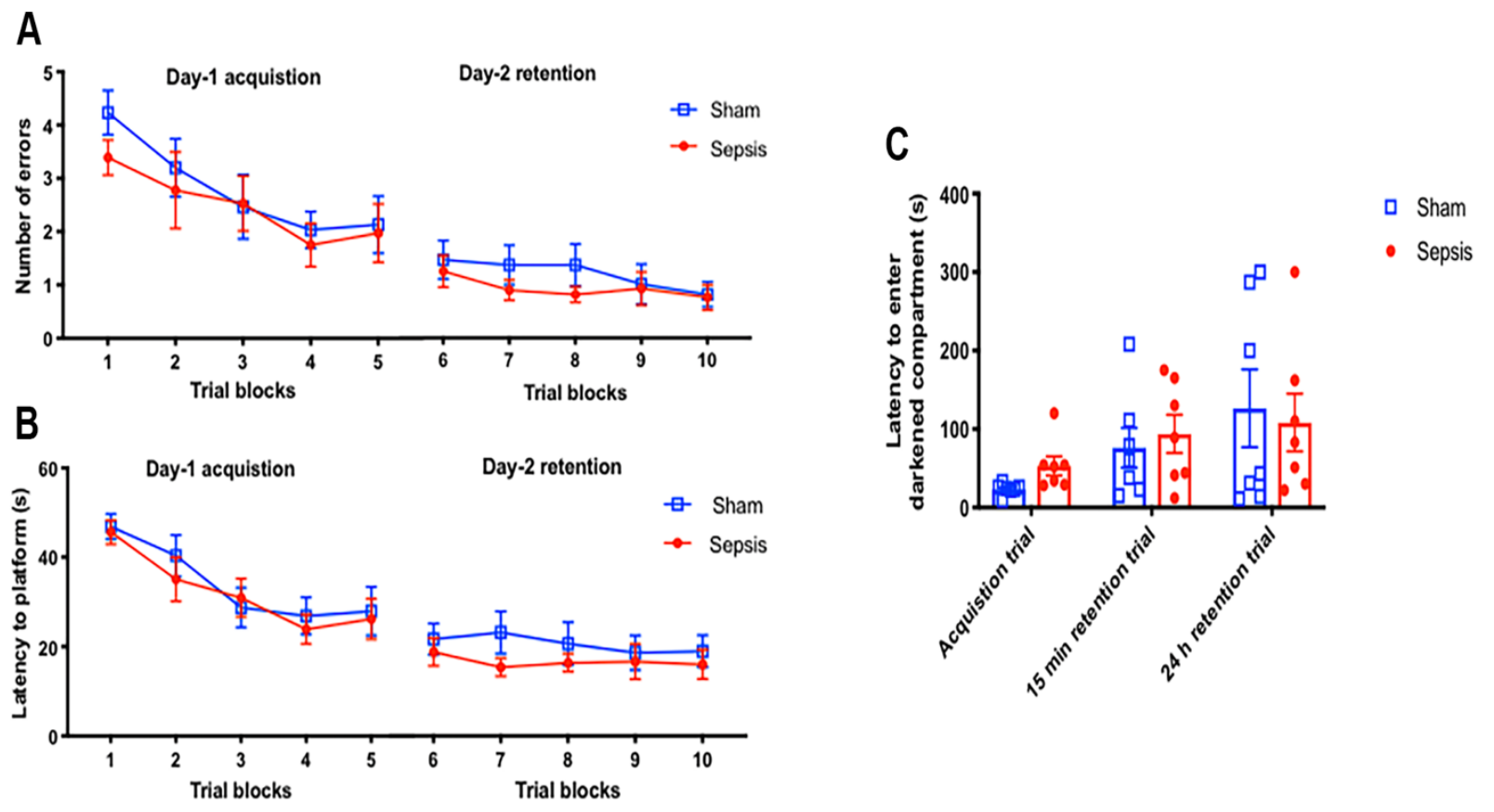


\section{Figure Legends}

Figure 1: Experimental design and behavioral testing paradigm to study neuroimmune and brain microvascular function in late sepsis. Animals in cohorts 1 and 2 were randomized and underwent either sham or sepsis (cecal ligation and puncture, CLP) surgery. All animals were subjected to a series of daily behavioral assays from day two to six. Mice were euthanized on day seven and their brains were harvested for flow cytometry (cohort 1) or immunohistochemistry (IHC), and alkaline phosphatase (AP) histology (cohort 2). Spinal cords were used for IHC and AP histology (cohort 2). Animals in cohort 3 underwent sepsis surgery alone and were injected with SBI-425 or vehicle intraperitoneally $1 \mathrm{~h}$ post-CLP and daily for six days post-CLP. Mice in cohort 3 were euthanized on day seven post-sepsis and brains collected for IHC.

Figure 2: Survival is decreased while longitudinal clinical scores and serum proinflammatory cytokines are increased in sepsis. (A) A Kaplan-Meier log-rank survival curve analysis of sham $(n=22)$ and septic $(n=38)$ mice from day 0 of surgery to euthanasia at day seven showed that septic mice had a significant decrease in survival $\left({ }^{* * *} p=0.001\right)$ compared to sham mice. $(B, C)$ Septic mice $(n=16)$ had significantly higher sickness scores $\left({ }^{* * *} p<0.0001\right.$, repeated two-way ANOVA) and average weight loss scores $\left({ }^{* * *} p=0.0005\right.$, Mann-Whitney test) compared to sham mice $(n=12)$. (D) Examination of blood serum inflammatory cytokines at day seven revealed a persistent increase in IL-6 ( ${ }^{* *} p=0.005$, MannWhitney test) and MCP-1 ( ${ }^{*} p=0.01$, Mann-Whitney test) in septic mice $(n=7)$ compared to sham mice $(\mathrm{n}=4-5)$. ${ }^{*}$ indicates $p<0.05,{ }^{* *} p<0.01,{ }^{* * *} p<0.001,{ }^{* * *} p<0.0001$, and is considered significant. All data are presented as mean \pm SEM.

Figure 3: Tissue nonspecific alkaline phosphatase (TNAP) enzyme activity in cerebral microvessels is decreased in late sepsis. (A) Representative images of TNAP enzyme activity on brain microvessels showed that TNAP's enzyme activity in the somatosensory cortex (20X magnification) of septic mice is decreased compared to sham mice. (B) Quantification of TNAP enzyme activity on sections of the somatosensory cortex showed that TNAP enzyme activity is significantly decreased $\left({ }^{*} p=0.02\right.$, unpaired t-test) in septic ( $\left.n=6\right)$ compared to sham treated mice $(n=5)$. (C) Co-labelling of TNAP enzyme activity (purple) with CD31 cerebral microvessel marker (brown) in the somatosensory cortex (10X and 40X magnification) further demonstrates that loss of TNAP's enzyme activity in brain sections is due to a loss of TNAP enzyme activity in cerebral microvessels rather than a loss of brain microvessels. * indicates $p<$ 0.05 , and is considered significant. All data are presented as mean \pm SEM. 10X, 20X, and 40X magnification scale bar $=160 \mu \mathrm{m}, 80 \mu \mathrm{m}$, and $40 \mu \mathrm{m}$ respectively.

Figure 4: Changes in claudin-5 expression and lgG permeability are consistent with diminished tissue nonspecific alkaline phosphatase (TNAP) enzyme activity in cerebral microvessels. (A) Normalized cell index is an impedance-based measure of barrier integrity. Treatment of hCMEC/D3 cells with TNAPI significantly decreased barrier integrity $\left({ }^{*} p=0.026\right.$, Tukey's multiple comparison test, two-way ANOVA) compared to DMSO treated hCMEC/D3 cells. Combination treatment of LPS and TNAPI significantly decreased barrier integrity compared to TNAPI only ( ${ }^{* * * *} p<0.0001$, Tukey's multiple comparison test, two-way ANOVA), LPS only $\left({ }^{* * * *} p<0.0001\right.$, Tukey's multiple comparison test, two-way ANOVA), and DMSO $\left({ }^{* * *} p<0.0001\right.$, Tukey's multiple comparison test, two-way ANOVA) treated hCMEC/D3 cells. (B) Claudin-5 (red) expression surrounding CD31 (brown) labelled brain microvessels is decreased in septic compared to sham mice (20X magnification). (C) Daily injections of SBI-425 up to day six post-sepsis dramatically reduced claudin-5 expression compared to vehicle (Veh) treated septic mice or naïve mice. $20 \mathrm{X}$ and $40 \mathrm{X}$ magnification scale bar $=80 \mu \mathrm{m}$ and $40 \mu \mathrm{m}$ 
respectively. (D) Vascular (cyan arrow) and perivascular IgG permeability (red arrow) is increased in septic brains compared to sham brains (white arrow) (40X magnification). Also, note that cortical microvessels with slight TNAP enzyme activity (green arrow) in septic brains showed reduced perivascular IgG permeability compared to areas without TNAP enzyme activity (red arrow) (40X magnification). ${ }^{*}$ indicates $p<0.05,{ }^{* * * *} p<0.0001$, and is considered significant. All data are presented as mean \pm SEM. 20X and $40 \mathrm{X}$ magnification scale bar $=80$ $\mu \mathrm{m}$ and $40 \mu \mathrm{m}$ respectively.

Figure 5: Brain infiltrating myeloid cell populations are elevated in late sepsis. (A-D) Representative dot plot and quantification of inflammatory myeloid cells showed a significant increase in neutrophil ( ${ }^{* *} p=0.004$, Mann-Whitney test) and monocyte $\left({ }^{* *} p=0.004\right.$, MannWhitney test) cell populations in the brains of septic compared to sham mice. [85] Analyses of myeloid derived suppressor cells (MDSCs) showed that septic brains have a significant increase in polymorphonuclear-MDSC/PMN-MDSC ( ${ }^{* *} p=0.004$, Mann-Whitney test) and monocyticMDSCs/M-MDSC ( ${ }^{* *} p=0.004$, Mann-Whitney test) cell populations compared to sham mice. Flow cytometric results are expressed as the percentage of an indicated cell type in total CD45 live cells in septic mice $(n=6)$ or sham mice $(n=5)$. Analyzed population is indicated by the encircled gating. ${ }^{* *}$ indicates $p<0.01$, and is considered significant. All data are presented as mean \pm SEM.

Figure 6: Multiple T-lymphoid cell populations are elevated in the brain during late sepsis. (A-D) Representative dot plot and quantification of T-lymphoid cells revealed that septic brains have a significant increase in CD8+ $\left({ }^{* *} p=0.004\right.$, Mann-Whitney test $)$ and CD4+ T-cell ( ${ }^{*} p=0.046$, unpaired t-test) populations compared to sham mice. (E, F) Further analyses of a specific subset of CD4 ${ }^{+}$cells (T-regulatory cells, Tregs) using Foxp3 and CD25 markers revealed a significant increase in the percentage of Tregs $\left({ }^{* *} p=0.0011\right.$, unpaired t-test) in the brains of septic compared to sham mice. Quantification of flow cytometric results are expressed as the percentage of an indicated cell type in total CD45 live cells in septic mice $(n=6)$ or sham mice $(n=5)$. The analyzed population is indicated by the encircled gating. * indicates $p<$ $0.05,{ }^{* *} p<0.01$, and is considered significant. All data are presented as mean \pm SEM.

Figure 7: Late sepsis is characterized by increased microglia/infiltrating monocyte populations. (A-F) Representative histological images (A, C, E) and quantification of Iba-1 (B, $D, F)$ showed an increased number of Iba-1 immunoreactive cells in the somatosensory cortex $\left({ }^{* *} p=0.004\right.$, Mann-Whitney test), CA1 $\left({ }^{* *} p=0.007\right.$, unpaired t-test) and CA3 $\left({ }^{* *} p=0.004\right.$, unpaired t-test) brain regions of septic $(n=6)$ compared to sham mice $(n=5)$. ${ }^{* *}$ indicates $p<$ 0.01 , and is considered significant. All data are presented as mean \pm SEM. 20X and 40X magnification scale bar $=80 \mu \mathrm{m}$, and $40 \mu \mathrm{m}$ respectively.

Figure 8: Sustained astrogliosis in the somatosensory cortex of septic mice. (A-F) Representative glial fibrillary acidic protein (GFAP) astrocyte histological images and quantification of astrogliosis (astrocyte proliferation) in the somatosensory cortex, CA1, and CA3 brain regions of septic and sham mice. (B) Quantification of GFAP astrocytes showed a sustained significant increase in astrocyte proliferation in the somatosensory cortex $\left({ }^{* *} p=\right.$ 0.006, unpaired t-test) of septic $(n=6)$ compared to sham mice $(n=5) .(D, F)$ However, no differences in astrocyte proliferation were seen between septic $(n=6)$ and sham mice $(n=6)$ in the hippocampal brain regions CA1 $(p=0.61$, unpaired t-test) and CA3 ( $p=0.56$, unpaired ttest). ${ }^{* *}$ indicates $p<0.01$, and is considered significant. All data are presented as mean \pm SEM. 20X and $40 \mathrm{X}$ magnification scale bar $=80 \mu \mathrm{m}$, and $40 \mu \mathrm{m}$ respectively. 
Figure 9: Spontaneous sensorimotor activity and nociceptive functions are impaired in septic mice. (A, B) Open field testing of spontaneous locomotion on day two showed that septic mice $(n=14)$ had a significant decrease in horizontal $\left({ }^{* * *} p<0.0001\right.$, repeated two-way ANOVA) and vertical $\left({ }^{* * *} p<0.0001\right.$, repeated two-way ANOVA) spontaneous locomotion compared to sham mice $(n=10)$. (C) Rotarod assessment of evoked locomotion on day three showed no difference ( $p=0.23$, repeated two-way ANOVA) between septic mice $(n=8)$ and sham mice $(n$ $=7)$. $(\mathrm{D}, \mathrm{E})$ Evaluation of sensory dysfunction with the hot plate test revealed a novel antinociceptive behavior in septic mice i.e., septic mice exhibited a significant increase in the latency to respond to thermal stimuli ( ${ }^{* *} p=0.01$, unpaired t-test) and a significant decrease in the total number of nociceptive behaviors (hind limb - lick, flick, and jump) $\left({ }^{* *} p=0.0096\right.$, unpaired t-test). ${ }^{* *}$ indicates $p<0.01,{ }^{* * * *} p<0.0001$, and is considered significant. All data are presented as mean \pm SEM.

Figure 10: Absence of spatial and non-spatial learning and memory behavioral deficits in late sepsis. (A, B) Mice underwent two-day radial arm water maze (2D-RAWM) testing to assess spatial learning and memory deficits on days five and six post-sepsis. Septic mice $(n=$ 12) showed no difference in the number of errors $(p=0.39$, repeated two-way ANOVA) or the latency to find the hidden platform ( $p=0.36$, repeated two-way ANOVA) on day two of the retention trial compared to sham mice $(n=10)$. (C) The passive avoidance assessed nonspatial learning and memory on days four and five post-sepsis. Septic mice $(n=7)$ showed no difference in latency to enter the darkened compartment ( $p=0.77$, repeated two-way ANOVA) compared to sham mice $(n=7)$. All data are presented as mean \pm SEM. 


\section{Supplementary Tables:}

\section{Supplementary Table 1}

Quantification of TMEM 119 positive brain microglia populations sham $(n=5)$ and septic mice $(n$ $=6)$

\begin{tabular}{|c|c|c|c|}
\hline \multicolumn{4}{|c|}{ Area of stained TMEM119 microglia/ $\mu \mathrm{m}^{2}$} \\
\hline \multicolumn{4}{|c|}{ Treatment } \\
\hline Brain region & Sham (mean \pm SEM) & Sepsis (mean \pm SEM) & p-value \\
\hline Cortex & $17634 \pm 2313$ & $27110 \pm 4924$ & 0.13 \\
\hline CA1 & $16152 \pm 1138$ & $16006 \pm 1609$ & 0.94 \\
\hline CA3 & $11837 \pm 2436$ & $10475 \pm 2103$ & 0.68 \\
\hline
\end{tabular}

All data are presented as mean \pm SEM, unpaired t-test.

\section{Supplementary Table 2}

Quantification of cholinergic ChAT positive neurons in the basal forebrain of sham $(n=5)$ and septic mice $(n=6)$

\begin{tabular}{|c|c|c|c|}
\hline \multicolumn{4}{|c|}{ \# of ChAT positive neurons } \\
\hline \multicolumn{4}{|c|}{ Treatment } \\
\hline Brain region & Sham (mean \pm SEM) & Sepsis (mean \pm SEM) & p-value \\
\hline Basal forebrain & $24 \pm 2.63$ & $19.4 \pm 3.04$ & 0.28 \\
\hline
\end{tabular}

All data are presented as mean \pm SEM, unpaired t-test. 
Supplemental Figures:

Supplemental Figure 1.

A.
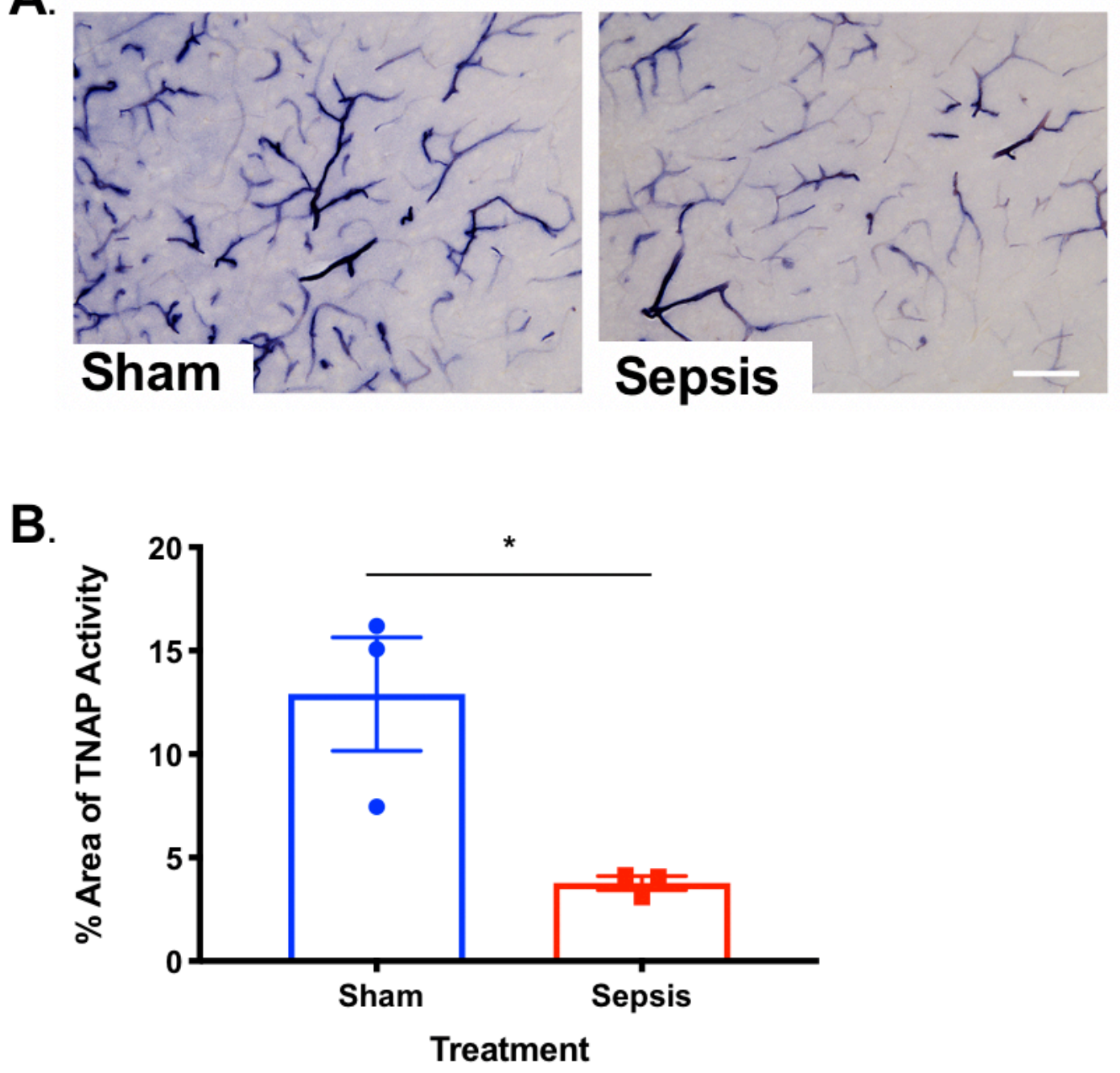
Supplementary Figure 2.
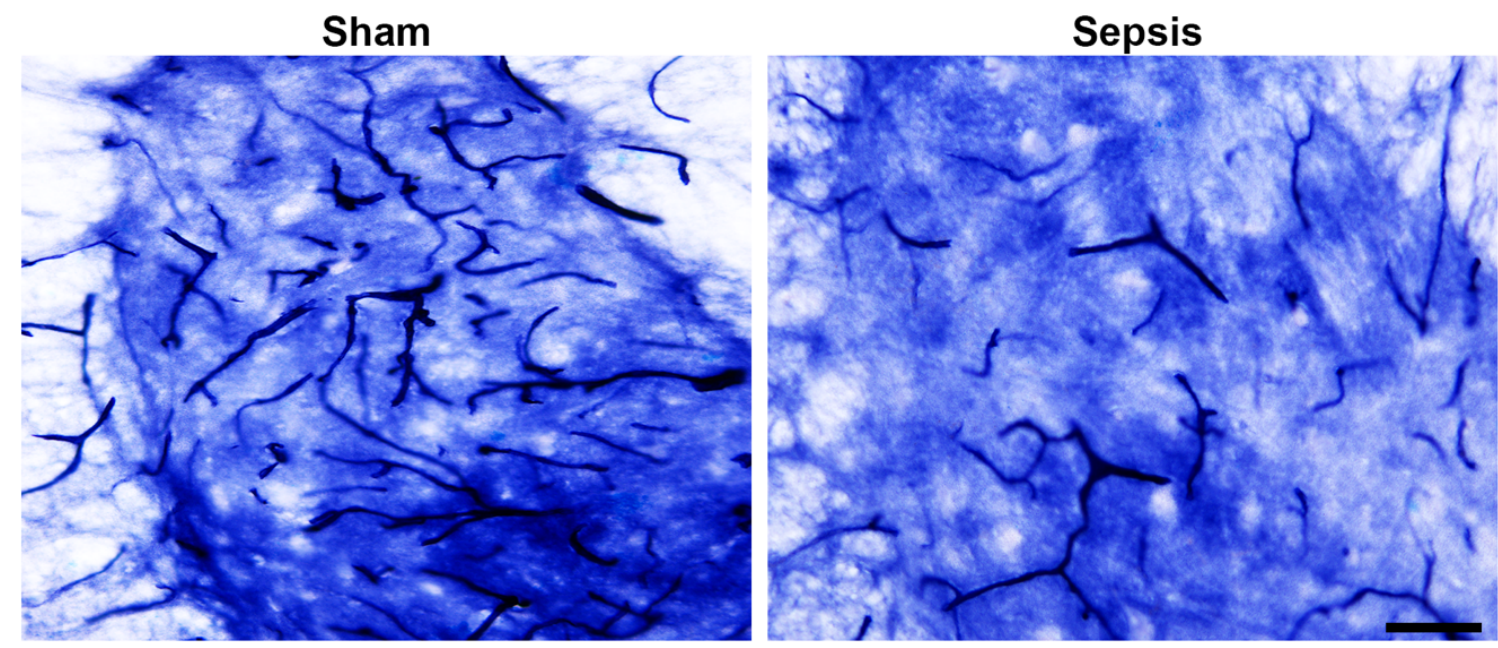
Supplementary Figure 3.

A Live cell gating
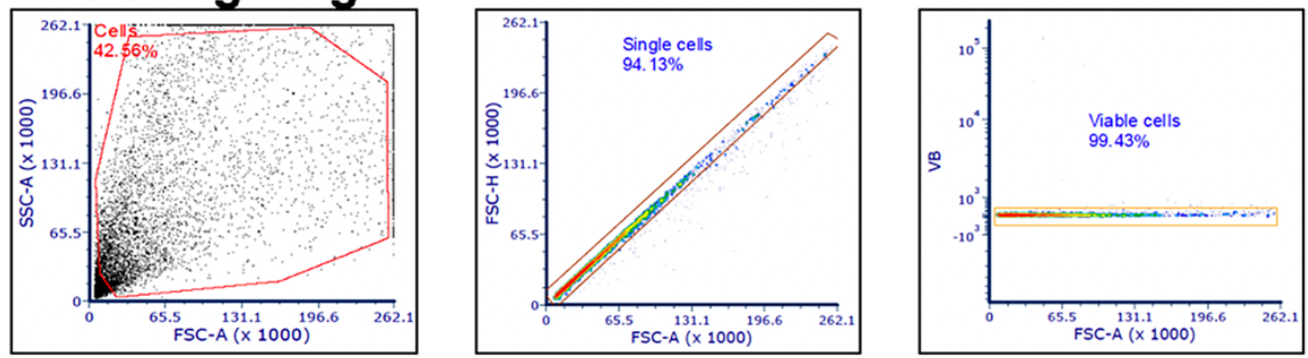

B Neutrophil
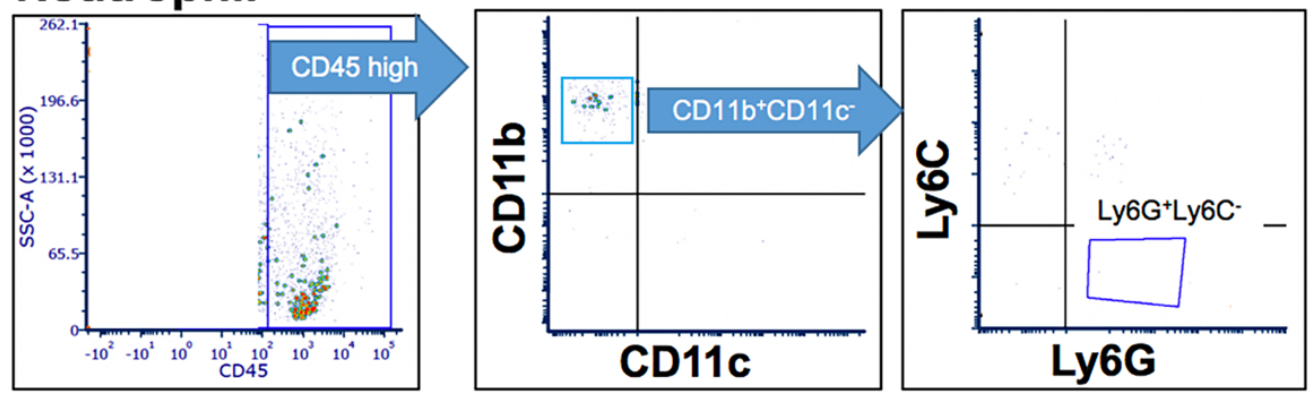

C Monocytes
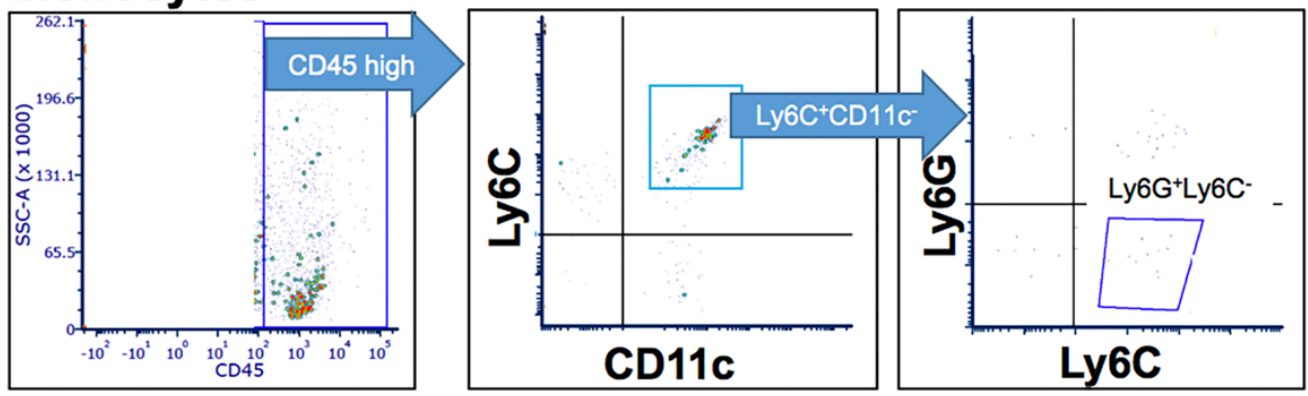

D MDSCs
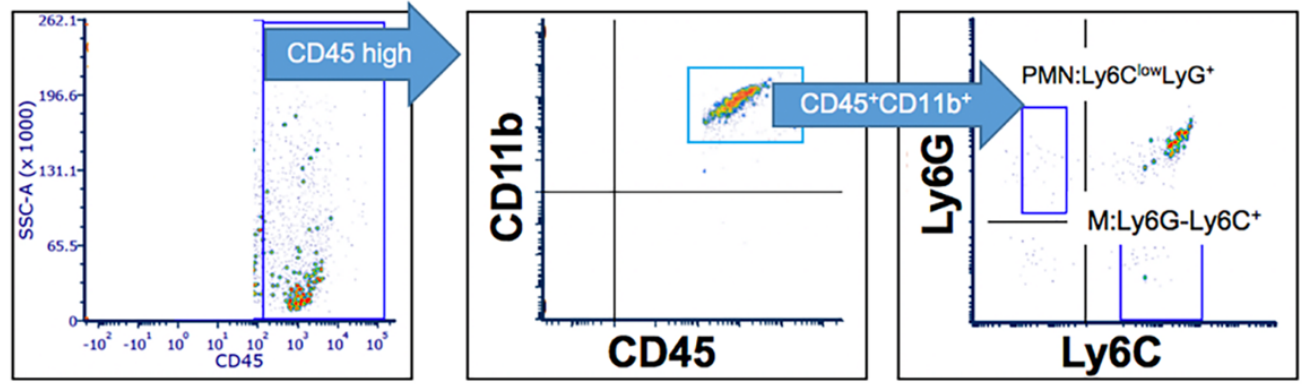
Supplementary Figure 4.

\section{A Live cell gating}
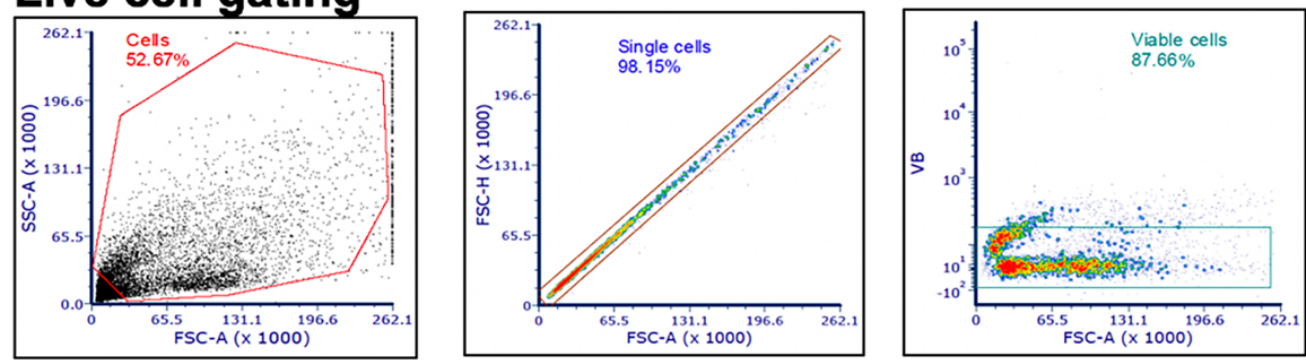

B CD4 T-cells

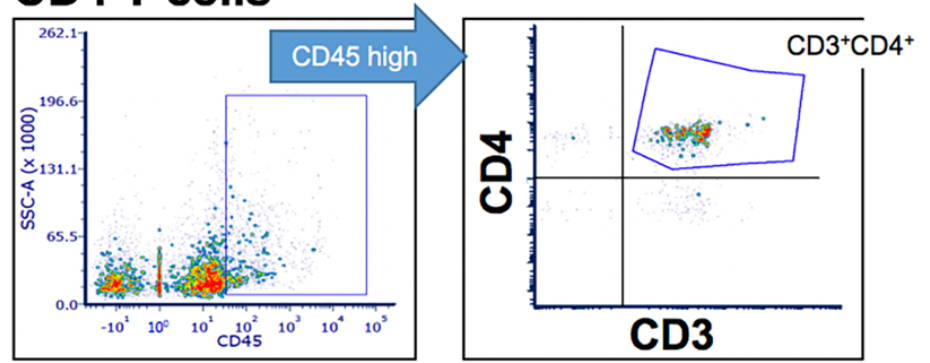

\section{CD8 T-cells}

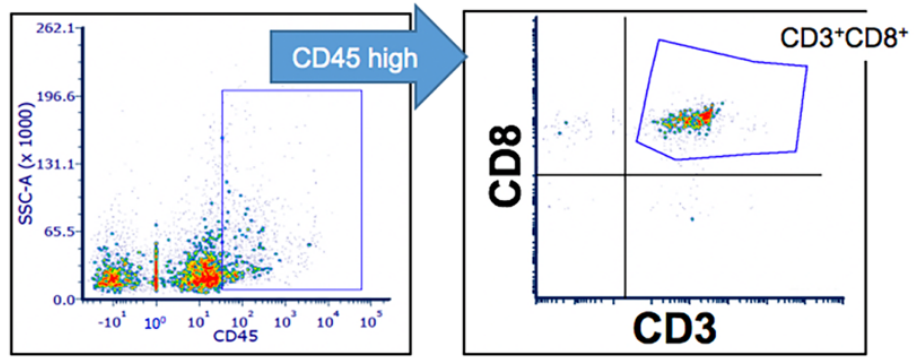

D T-regulatory cells

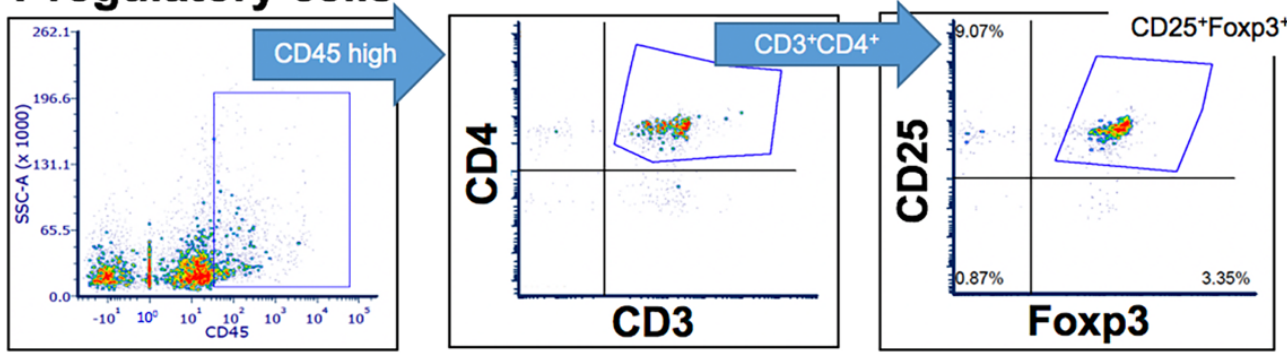


Supplementary Figure 5.
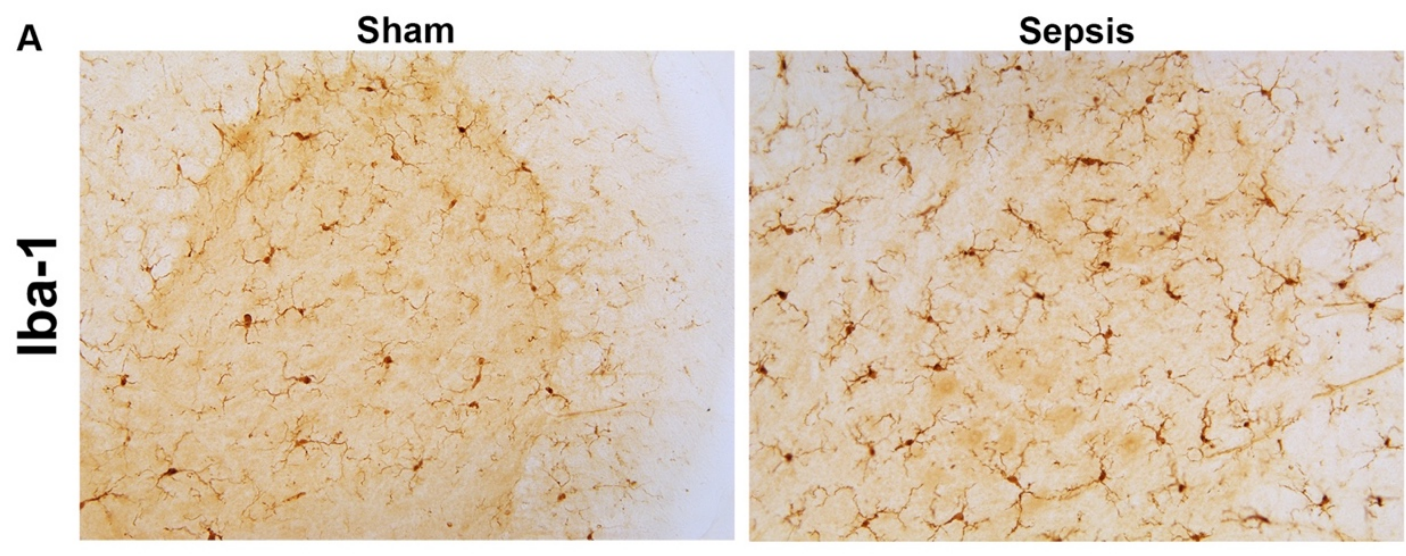

B
$\frac{1}{4}$
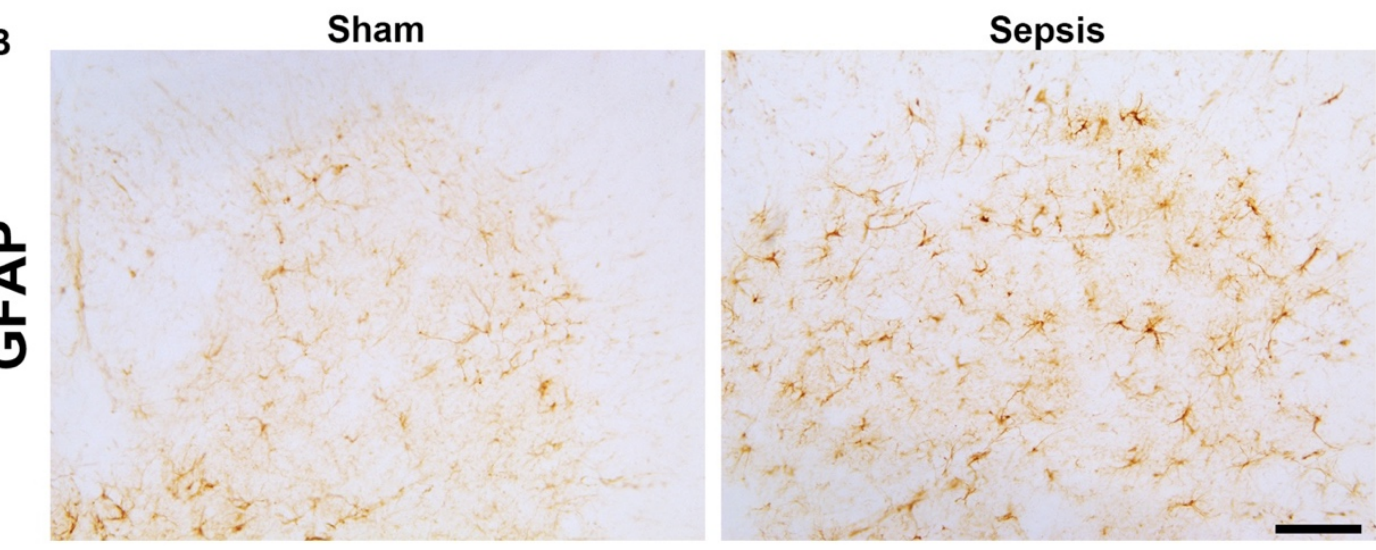
Supplementary Figure 6.
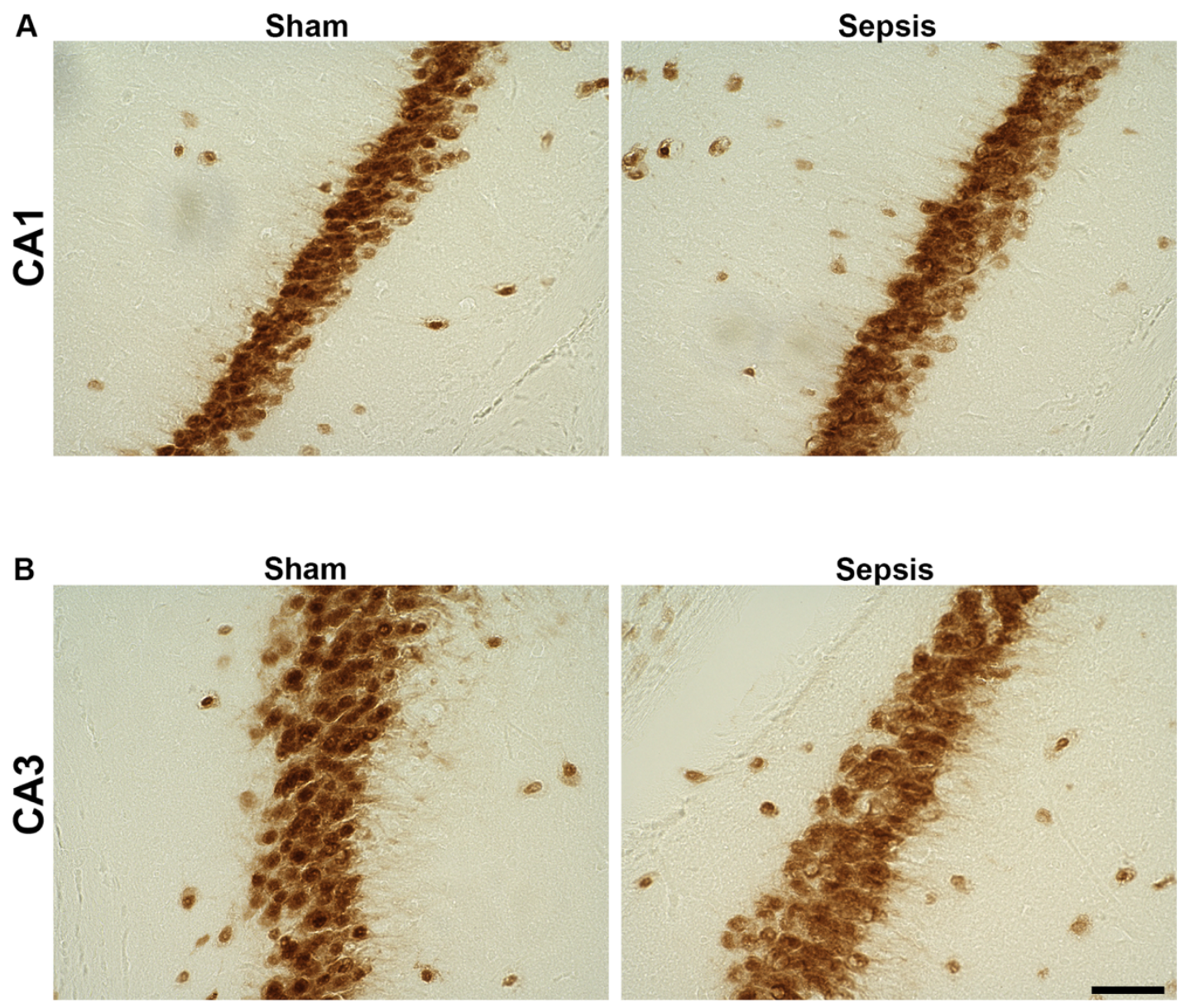


\section{Supplementary Figure Legends}

\section{Supplementary Figure 1.}

TNAP activity in the brain $24 \mathrm{~h}$ post-sepsis. (A) Histological staining for TNAP activity shows decreased TNAP enzyme activity in the cortex of septic male mice subjected to the cecal ligation and puncture (CLP) model of experimental sepsis. C57BL/6J mice were subjected to CLP or a sham injury and brains were harvested 24 hours later. (B) Graph shows the quantification of cortical AP enzyme activity in CLP $(n=3,52.49 \pm 0.1094)$ versus sham $(n=3$, $53 \pm 0.1142$ ) mice (sections $=3$ per mouse; data represented as mean $\pm \mathrm{SEM},{ }^{*} P<.05, t$ $(4)=3.384$, unpaired Student's $t$-test, scale bar $=115 \mu \mathrm{m})$.

\section{Supplementary Figure 2.}

Tissue non-specific alkaline phosphatase (TNAP) enzyme activity in the spinal cord. Representative alkaline phosphatase (AP) histology shows that TNAP's enzyme activity is decreased in the spinal cord of septic mice compared to sham mice (20X magnification). $20 \mathrm{X}$ magnification scale bar $=80 \mu \mathrm{m}$.

\section{Supplementary Figure 3.}

Gating strategy for brain myeloid populations. (A) Representative live cell gating strategy. Neutrophil (B), monocytes (C), and MDSCs (C) were gating under the parent gate CD45 ${ }^{\text {hi }}$ prior to being organized into precise cell types using the following specific markers: CD11b, CD11c, Ly6G, and Ly6C. SSC = side scatter, FSC = forward scatter, $A=$ area, $H=$ height, VB = viability, $\mathrm{M}=$ monocytic MDSCs, and PMN = polymorphonuclear MDSCs. Analyzed population is indicated by the encircled gating.

\section{Supplementary Figure 4.}

Gating strategy for brain lymphoid populations. (A) Representative live cell gating strategy. CD4, CD8, and regulatory T-cells were gating under the parent gate CD45 hi prior to being organized into precise cell types using the following specific markers: CD3, CD4, CD8, CD25, and Foxp3. SSC = side scatter, FSC = forward scatter, $A=$ area, $\mathrm{H}=$ height, and $\mathrm{VB}=$ viability. Analyzed population is indicated by the encircled gating.

\section{Supplementary Figure 5.}

Iba-1 positive microglia and GFAP positive astrocyte immunohistochemistry in the spinal cord of septic and sham mice. (A) lba-1 positive cells are increased in the spinal cords of septic mice compared to sham mice. (B) GFAP positive cells are increased in the spinal cords of septic mice compared to sham mice (20X magnification). 20X magnification scale bar $=80$ $\mu \mathrm{m}$.

\section{Supplementary Figure 6.}

NeuN positive immunohistochemistry for neuronal loss in the CA1 and CA3 hippocampal regions. Representative histology for NeuN immunolabelling shows no apparent differences in neuronal loss in the (A) CA1 or (C) CA3 between septic and sham mice (40X magnification). 40X magnification scale bar $=40 \mu \mathrm{m}$. 


\section{References}

1. Stare, J., et al., Effects of Peritoneal Sepsis on Rat Central Osmoregulatory Neurons Mediating Thirst and Vasopressin Release. J Neurosci, 2015. 35(35): p. 12188-97.

2. Zaghloul, N., et al., Forebrain Cholinergic Dysfunction and Systemic and Brain Inflammation in Murine Sepsis Survivors. Front Immunol, 2017. 8: p. 1673.

3. Varatharaj, A. and I. Galea, The blood-brain barrier in systemic inflammation. Brain Behav Immun, 2017. 60: p. 1-12.

4. Fleischmann, C., et al., Assessment of Global Incidence and Mortality of Hospital-treated Sepsis. Current Estimates and Limitations. Am J Respir Crit Care Med, 2016. 193(3): p. 259-72.

5. Brichacek, A.L., et al., Systemic inhibition of tissue-nonspecific alkaline phosphatase alters the brain-immune axis in experimental sepsis. Sci Rep, 2019. 9(1): p. 18788.

6. Boehme, A.K., et al., Risk of Acute Stroke After Hospitalization for Sepsis: A CaseCrossover Study. Stroke, 2017. 48(3): p. 574-580.

7. Wang, H.E., et al., Chronic medical conditions and risk of sepsis. PLoS One, 2012. 7(10): p. e48307.

8. Ovbiagele, B., et al., Forecasting the future of stroke in the United States: a policy statement from the American Heart Association and American Stroke Association. Stroke, 2013. 44(8): p. 2361-75.

9. Stoller, J., et al., Epidemiology of severe sepsis: 2008-2012. J Crit Care, 2016. 31(1): p. 58-62.

10. Fink, M.P. and H.S. Warren, Strategies to improve drug development for sepsis. Nat Rev Drug Discov, 2014. 13(10): p. 741-58.

11. Iskander, K.N., et al., Sepsis: multiple abnormalities, heterogeneous responses, and evolving understanding. Physiol Rev, 2013. 93(3): p. 1247-88.

12. Iwashyna, T.J., et al., Long-term cognitive impairment and functional disability among survivors of severe sepsis. JAMA, 2010. 304(16): p. 1787-94.

13. Zauner, C., et al., Impaired subcortical and cortical sensory evoked potential pathways in septic patients. Crit Care Med, 2002. 30(5): p. 1136-9.

14. Gofton, T.E. and G.B. Young, Sepsis-associated encephalopathy. Nat Rev Neurol, 2012. 8(10): p. 557-66.

15. Singer, B.H., et al., Cecal Ligation and Puncture Results in Long-Term Central Nervous System Myeloid Inflammation. PLoS One, 2016. 11(2): p. e0149136.

16. Andonegui, G., et al., Targeting inflammatory monocytes in sepsis-associated encephalopathy and long-term cognitive impairment. JCI Insight, 2018. 3(9).

17. Hollenbeck, P.J., et al., Intermediate filament collapse is an ATP-dependent and actindependent process. J Cell Sci, 1989. 92 ( Pt 4): p. 621-31.

18. Wang, P., et al., Omi/HtrA2 Regulates a Mitochondria-Dependent Apoptotic Pathway in a Murine Model of Septic Encephalopathy. Cell Physiol Biochem, 2018. 49(6): p. 21632173.

19. Towner, R.A., et al., Assessing long-term neuroinflammatory responses to encephalopathy using MRI approaches in a rat endotoxemia model. Geroscience, 2018. 40(1): p. 49-60.

20. Deracinois, B., et al., Tissue Non-specific Alkaline Phosphatase (TNAP) in Vessels of the Brain. Subcell Biochem, 2015. 76: p. 125-51.

21. Millan, J.L., Mammalian alkaline phosphatases : from biology to applications in medicine and biotechnology. 2006, Weinheim: Wiley-VCH.

22. Bender, B., et al., Recombinant human tissue non-specific alkaline phosphatase successfully counteracts lipopolysaccharide induced sepsis in mice. Physiol Res, 2015. 64(5): p. 731-8. 
23. Verweij, W.R., et al., Protection against an Escherichia coli-induced sepsis by alkaline phosphatase in mice. Shock, 2004. 22(2): p. 174-9.

24. Vorbrodt, A.W., A.S. Lossinsky, and H.M. Wisniewski, Localization of alkaline phosphatase activity in endothelia of developing and mature mouse blood-brain barrier. Dev Neurosci, 1986. 8(1): p. 1-13.

25. Betz, A.L., J.A. Firth, and G.W. Goldstein, Polarity of the blood-brain barrier: distribution of enzymes between the luminal and antiluminal membranes of brain capillary endothelial cells. Brain Res, 1980. 192(1): p. 17-28.

26. Friede, R.L., A quantitative mapping of alkaline phosphatase in the brain of the rhesus monkey. J Neurochem, 1966. 13(3): p. 197-203.

27. Otto, G.P., et al., The late phase of sepsis is characterized by an increased microbiological burden and death rate. Crit Care, 2011. 15(4): p. R183.

28. Crowell, K.T., et al., Immune and metabolic responses in early and late sepsis during mild dietary zinc restriction. J Surg Res, 2017. 210: p. 47-58.

29. Wang, X., et al., Sirtuin-2 Regulates Sepsis Inflammation in ob/ob Mice. PLoS One, 2016. 11(8): p. e0160431.

30. Vachharajani, V.T., et al., SIRT1 inhibition during the hypoinflammatory phenotype of sepsis enhances immunity and improves outcome. J Leukoc Biol, 2014. 96(5): p. 78596.

31. Pinkerton, A.B., et al., Discovery of 5-((5-chloro-2methoxyphenyl)sulfonamido)nicotinamide (SBI-425), a potent and orally bioavailable tissue-nonspecific alkaline phosphatase (TNAP) inhibitor. Bioorg Med Chem Lett, 2018. 28(1): p. 31-34.

32. Weksler, B.B., et al., Blood-brain barrier-specific properties of a human adult brain endothelial cell line. FASEB J, 2005. 19(13): p. 1872-4.

33. Sheen, C.R., et al., Pathophysiological role of vascular smooth muscle alkaline phosphatase in medial artery calcification. J Bone Miner Res, 2015. 30(5): p. 824-36.

34. Shrum, B., et al., A robust scoring system to evaluate sepsis severity in an animal model. BMC Res Notes, 2014. 7: p. 233.

35. Bachman, J., Immunohistochemistry on freely floating fixed tissue sections. Methods Enzymol, 2013. 533: p. 207-15.

36. Eriksson, J.E., et al., Introducing intermediate filaments: from discovery to disease. J Clin Invest, 2009. 119(7): p. 1763-71.

37. Garcia-Bonilla, L., et al., Endogenous Protection from Ischemic Brain Injury by Preconditioned Monocytes. J Neurosci, 2018. 38(30): p. 6722-6736.

38. Pratt, W.B. and D.O. Toft, Steroid receptor interactions with heat shock protein and immunophilin chaperones. Endocr Rev, 1997. 18(3): p. 306-60.

39. Doll, D.N., et al., Lipopolysaccharide exacerbates infarct size and results in worsened post-stroke behavioral outcomes. Behav Brain Funct, 2015. 11(1): p. 32.

40. Barichello, T., et al., Cognitive impairment in sepsis survivors from cecal ligation and perforation. Crit Care Med, 2005. 33(1): p. 221-3; discussion 262-3.

41. Vachon, P., et al., Alleviation of chronic neuropathic pain by environmental enrichment in mice well after the establishment of chronic pain. Behav Brain Funct, 2013. 9: p. 22.

42. Colton, C.A., et al., mNos2 deletion and human NOS2 replacement in Alzheimer disease models. J Neuropathol Exp Neurol, 2014. 73(8): p. 752-69.

43. Kan, M.J., et al., Arginine deprivation and immune suppression in a mouse model of Alzheimer's disease. J Neurosci, 2015. 35(15): p. 5969-82.

44. Kilkenny, C., et al., Animal research: reporting in vivo experiments: the ARRIVE guidelines. Br J Pharmacol, 2010. 160(7): p. 1577-9.

45. Angele, M.K., et al., Gender differences in sepsis: cardiovascular and immunological aspects. Virulence, 2014. 5(1): p. 12-9. 
46. Denstaedt, S.J., et al., S100A8/A9 Drives Neuroinflammatory Priming and Protects against Anxiety-like Behavior after Sepsis. J Immunol, 2018. 200(9): p. 3188-3200.

47. Cardoso, F.L., et al., Exposure to lipopolysaccharide and/or unconjugated bilirubin impair the integrity and function of brain microvascular endothelial cells. PLoS One, 2012. 7(5): p. e35919.

48. Hu, Y., et al., Omi/HtrA2 Protease Associated Cell Apoptosis Participates in Blood-Brain Barrier Dysfunction. Front Mol Neurosci, 2019. 12: p. 48.

49. Brun-Heath, I., et al., Differential expression of the bone and the liver tissue non-specific alkaline phosphatase isoforms in brain tissues. Cell Tissue Res, 2011. 343(3): p. 521-36.

50. Street, S.E., et al., Tissue-nonspecific alkaline phosphatase acts redundantly with PAP and NT5E to generate adenosine in the dorsal spinal cord. J Neurosci, 2013. 33(27): p. 11314-22.

51. Nowak, L.G., et al., Tetramisole and Levamisole Suppress Neuronal Activity Independently from Their Inhibitory Action on Tissue Non-specific Alkaline Phosphatase in Mouse Cortex. Subcell Biochem, 2015. 76: p. 239-81.

52. Debray, J., et al., Inhibitors of tissue-nonspecific alkaline phosphatase: design, synthesis, kinetics, biomineralization and cellular tests. Bioorg Med Chem, 2013. 21(24): p. 7981-7.

53. Yang, C.H., et al., Simvastatin attenuates sepsis-induced blood-brain barrier integrity loss. J Surg Res, 2015. 194(2): p. 591-8.

54. Banks, W.A., et al., Lipopolysaccharide-induced blood-brain barrier disruption: roles of cyclooxygenase, oxidative stress, neuroinflammation, and elements of the neurovascular unit. J Neuroinflammation, 2015. 12: p. 223.

55. Bennett, M.L., et al., New tools for studying microglia in the mouse and human CNS. Proc Natl Acad Sci U S A, 2016. 113(12): p. E1738-46.

56. Khan, J., et al., Early development of critical illness myopathy and neuropathy in patients with severe sepsis. Neurology, 2006. 67(8): p. 1421-5.

57. Widmann, C.N. and M.T. Heneka, Long-term cerebral consequences of sepsis. Lancet Neurol, 2014. 13(6): p. 630-6.

58. Mazeraud, A., et al., Neuroanatomy and Physiology of Brain Dysfunction in Sepsis. Clin Chest Med, 2016. 37(2): p. 333-45.

59. Sonneville, R., et al., Understanding brain dysfunction in sepsis. Ann Intensive Care, 2013. 3(1): p. 15.

60. Bi, W., et al., USP8 ameliorates cognitive and motor impairments via microglial inhibition in a mouse model of sepsis-associated encephalopathy. Brain Res, 2019.

61. Buchet, R., J.L. Millan, and D. Magne, Multisystemic functions of alkaline phosphatases. Methods Mol Biol, 2013. 1053: p. 27-51.

62. Helms, H.C., et al., In vitro models of the blood-brain barrier: An overview of commonly used brain endothelial cell culture models and guidelines for their use. J Cereb Blood Flow Metab, 2016. 36(5): p. 862-90.

63. Langer, D., et al., Distribution of ectonucleotidases in the rodent brain revisited. Cell Tissue Res, 2008. 334(2): p. 199-217.

64. Hotchkiss, R.S., et al., The sepsis seesaw: tilting toward immunosuppression. Nat Med, 2009. 15(5): p. 496-7.

65. Deutschman, C.S. and K.J. Tracey, Sepsis: current dogma and new perspectives. Immunity, 2014. 40(4): p. 463-75.

66. Mira, J.C., et al., Persistent Inflammation, Immunosuppression and Catabolism Syndrome. Crit Care Clin, 2017. 33(2): p. 245-258.

67. Delano, M.J. and P.A. Ward, The immune system's role in sepsis progression, resolution, and long-term outcome. Immunol Rev, 2016. 274(1): p. 330-353. 
68. Molinaro, R., et al., CCR4 Controls the Suppressive Effects of Regulatory T Cells on Early and Late Events during Severe Sepsis. PLoS One, 2015. 10(7): p. e0133227.

69. Tatura, R., et al., Relevance of Foxp3(+) regulatory T cells for early and late phases of murine sepsis. Immunology, 2015. 146(1): p. 144-56.

70. Cao, C., et al., Toll-like receptor 4 deficiency increases resistance in sepsis-induced immune dysfunction. Int Immunopharmacol, 2018. 54: p. 169-176.

71. Hosomi, S., et al., Myeloid-Derived Suppressor Cells Infiltrate the Brain and Suppress Neuroinflammation in a Mouse Model of Focal Traumatic Brain Injury. Neuroscience, 2019. 406: p. 457-466.

72. Hoogland, I.C., et al., Systemic inflammation and microglial activation: systematic review of animal experiments. J Neuroinflammation, 2015. 12: p. 114.

73. Prinz, M., D. Erny, and N. Hagemeyer, Ontogeny and homeostasis of CNS myeloid cells. Nat Immunol, 2017. 18(4): p. 385-392.

74. Colton, C.A., Heterogeneity of microglial activation in the innate immune response in the brain. J Neuroimmune Pharmacol, 2009. 4(4): p. 399-418.

75. Smolders, S.M., et al., Microglia: Brain cells on the move. Prog Neurobiol, 2019. 178: p. 101612.

76. Wu, L., et al., Serum glial fibrillary acidic protein and ubiquitin C-terminal hydrolase-L1 for diagnosis of sepsis-associated encephalopathy and outcome prognostication. J Crit Care, 2019. 52: p. 172-179.

77. Ward, R.D., Methods for Dissecting Motivation and Related Psychological Processes in Rodents. Curr Top Behav Neurosci, 2016. 27: p. 451-70.

78. Anderson, S.T., et al., Lipopolysaccharide-induced sepsis induces long-lasting affective changes in the mouse. Brain Behav Immun, 2015. 43: p. 98-109.

79. Barichello, T., et al., Long-Term Cognitive Outcomes After Sepsis: a Translational Systematic Review. Mol Neurobiol, 2019. 56(1): p. 186-251.

80. Erbs, G.C., et al., Comorbidities Might Condition the Recovery of Quality of Life in Survivors of Sepsis. J Intensive Care Med, 2019. 34(4): p. 337-343.

81. Thomas, R., et al., Nociceptin system as a target in sepsis? J Anesth, 2014. 28(5): p. 759-67.

82. Karshikoff, B., et al., Modality and sex differences in pain sensitivity during human endotoxemia. Brain Behav Immun, 2015. 46: p. 35-43.

83. Garland, E.L., Pain processing in the human nervous system: a selective review of nociceptive and biobehavioral pathways. Prim Care, 2012. 39(3): p. 561-71.

84. Chavan, S.S., et al., HMGB1 mediates cognitive impairment in sepsis survivors. Mol Med, 2012. 18: p. 930-7.

85. Keime-Guibert, F., et al., Treatment of paraneoplastic neurological syndromes with antineuronal antibodies (Anti-Hu, anti-Yo) with a combination of immunoglobulins, cyclophosphamide, and methylprednisolone. J Neurol Neurosurg Psychiatry, 2000. 68(4): p. 479-82. 


\section{Chapter 3}

\section{Brain endothelial cell tissue-nonspecific alkaline phosphatase (TNAP) activity promotes maintenance of barrier impedance via the ROCK pathway}

Divine C. Nwafor ${ }^{a}$, Allison L. Brichacek ${ }^{b}$, Wei Wang $^{a}$, Nina Bidwaic ${ }^{c}$, Christa L. Lilly ${ }^{d}$, José Luis Millán ${ }^{\mathrm{e}}$, and Candice M. Brown ${ }^{\mathrm{a} \mathrm{b}^{*}}$

\footnotetext{
${ }^{a}$ Department of Neuroscience, West Virginia University Health Science Center, Morgantown, WV 26506, USA

${ }^{\mathrm{b}}$ Department of Microbiology, Immunology, and Cell Biology, School of Medicine, West Virginia University Health Science Center, Morgantown, WV 26506, USA

'West Virginia University School of Medicine, Morgantown, WV 26506, USA

${ }^{d}$ Department of Biostatistics, School of Public Health, West Virginia University Health Science Center, Morgantown, WV 26506, USA

eSanford-Burnham Prebys Medical Discovery Institute, La Jolla, CA 92037, USA
}

Nwafor DC, Brichacek AL, Wang W, Bidwai N, Lilly CL, Millan JL, Brown CM. Brain endothelial cell tissue-nonspecific alkaline phosphatase (TNAP) activity promotes loss of barrier integrity via the ROCK pathway. Submitted.

Note: Parts of this chapter include text and figures taken from the publication highlighted above 


\subsection{Abstract}

Blood-brain barrier (BBB) dysfunction is a key feature in many neuroinflammatory diseases. Yet, no therapies exist to effectively mitigate BBB dysfunction. A strategy to bridge this knowledge gap requires an examination of proteins localized to brain microvascular endothelial cells (BMECs) and evaluating their role in preserving barrier integrity. Tissuenonspecific alkaline phosphatase (TNAP) is highly abundant in brain microvascular endothelial cells (BMECs); however, its function in BMECs remains unclear. We hypothesized that a loss or inhibition of TNAP activity on BMECs would impair barrier integrity through increased cytoskeletal remodeling driven by the Rho-associated protein kinase [1] pathway. First, we examined barrier integrity (measured as impedance) in hCMEC/D3 cells treated with a TNAP inhibitor (TNAPi) and in primary BMECs (pBMECs) via the conditional deletion of TNAP in endothelial cells. Our results showed that both pharmacological inhibition and genetic conditional loss of TNAP significantly worsened endothelial barrier impedance compared to controls. Next, we examined the mechanisms through which TNAP activity exerts a protective phenotype on BMECs. Our results showed that hCMEC/D3 cells treated with TNAPi displayed remarkable phalloidin and vimentin cytoskeletal remodeling compared to control. We then examined the role of ROCK, a key player in cytoskeletal remodeling. Our results showed that TNAPi increased the expression of ROCK 1/2. Furthermore, inhibition of ROCK 1/2 with fasudil mitigated TNAPi-induced and VE-cKO barrier dysfunction. Collectively, our results support a novel mechanism through which loss of TNAP activity results in cerebrovascular dysfunction, and selective modulation of TNAP activity in BMECs may be a therapeutic strategy to improve BBB function.

Keywords: Tissue-nonspecific alkaline phosphatase; Alpl; Barrier integrity; ROCK; Brain microvasculature; Cytoskeleton 


\subsection{Introduction}

The blood-brain barrier (BBB) is a dynamic vascular interface that separates the brain parenchyma from systemic circulation [2]. The brain's microvasculature differs from the peripheral vasculature due to its unique composition of brain capillary endothelial cells linked tightly together by junctional proteins, surrounding pericytes, basal lamina, and astrocyte endfoot processes [3]. At the BBB, brain capillary endothelial cell-cell junctions are critical for maintaining the integrity of the BBB via paracellular transport size selective restriction of molecules, toxin, and cells [4]. Furthermore, the expression of specific proteins, enzymes, and transporters on luminal and abluminal surfaces of brain capillary endothelial cells help to regulate blood-to-brain trafficking of certain molecules [5]. One example of these important proteins is the endothelial sphingosine-1-receptor-1 (S1Pr $\left.\mathrm{P}_{1}\right)$. Yanagida et al., showed that conditional endothelial knockout of S1Pr1 led to a size selective BBB leakiness to fluorescent tracers less than $3 \mathrm{kDa}$. Furthermore, BBB leakiness in the S1Pr1 endothelial knockout mice was coupled to altered subcellular distribution of junctional proteins [6]. Thus, a crossexamination of specific proteins, enzymes, and transporters localized to brain capillary endothelial cells may provide therapeutic insight that may mitigate BBB dysfunction and the consequential long-term cognitive impairment seen across many neuroinflammatory conditions $[3,7]$.

Alkaline phosphatases [8] are found in numerous tissues, and are thought to play an important role in regulating inflammation [9]. There are four AP isozymes in humans (gene name in italics): placental alkaline phosphatase (PLAP; $A L P P)$, germ cell alkaline phosphatase (GCAP; ALPPL2), intestinal alkaline phosphatase (IAP; $A L P I)$, and tissue-nonspecific alkaline phosphatase (TNAP; ALPL; also known as Akp2 or Alpl in mouse) [10]. Of the four AP isozymes, only TNAP is expressed in the brain tissue of humans and rodents [11]. Moreover, biochemical and histological studies have shown that brain microvessels are abundantly rich in TNAP $[12,13]$. Several studies have demonstrated a role for APs in catalyzing the hydrolysis of 
nucleotides (ATP, ADP, and AMP) to free adenosine and inorganic phosphates [14]. Similarly, APs are able to dephosphorylate inflammatory molecules such as lipopolysaccharide (LPS), damage-associated molecular patterns (DAMPs), and pathogen-associated molecular patterns (PAMPs) [15-17]. Free adenosine and dephosphorylation of LPS (dLPS) are suggested to support the anti-inflammatory role for APs in disease [14, 18-20]. However, the molecular mechanisms through which APs maintain homeostasis via its putative anti-inflammatory function remain unclear. Earlier investigations from our group revealed that brain microvascular TNAP activity is decreased as early as $24 \mathrm{~h}$ post-sepsis, and this decrease is sustained up to 7 days post-sepsis. Furthermore, the decrease in brain microvascular TNAP activity at 7-days postsepsis was coupled to increased immunoglobulin G [21] permeability and sustained neuroinflammation. Moreover, treatment of septic animals with a specific TNAP inhibitor decreased brain endothelial junctional protein claudin- 5 expression compared to vehicle treated septic and naïve mice $[3,22]$. These results suggest a putative role for microvascular TNAP activity in maintaining vascular barrier integrity at the endothelium. Therefore, in this study we elucidated the mechanistic role for TNAP at the brain endothelium and examined the molecular and cellular pathways targeted by TNAP.

Investigating the role of brain endothelial TNAP has been challenging for decades due to TNAP's ubiquitous expression in other tissue types such as the liver, kidney, spleen, lung, bone, and diverse brain cell types $[10,23]$. Furthermore, mice with a global knockout of TNAP $\left(A / p r^{-1}\right)$ die within days after birth from seizures and rickets characteristics of hypophosphatasia [24]. Newly generated pharmacological agents such as the TNAP inhibitor (TNAPi) probe and the pharmacological derivative 5-((5-chloro-2-methoxyphenyl) sulfonamido) nicotinamide (SBI-425) have advanced our knowledge on TNAP function in recent years [25, 26]; however, the use of these drugs in vivo makes it difficult to delineate brain endothelial TNAP function as opposed to other cell types. The availability of the Alp/ floxed mouse allowed for a targeted approach to delineate cell-specific function by Cre-lox recombination [27]. We utilized both pharmacological 
(using TNAP inhibitor (TNAPi)) and genetic TNAP deletion on endothelial cells (i.e., VE-cKO mice), to demonstrate a mechanistic role for brain endothelial TNAP in maintaining barrier integrity (measured as impedance) in vitro and ex vivo. Taken together, our results suggest that TNAP plays a critical role in maintaining in vitro barrier impedance via Rho-associated protein kinase mediated by cytoskeletal reorganization.

\subsection{Methods}

\subsubsection{Cell lines}

The hCMEC/D3 human cerebral microvascular endothelial cell line (D3 cells) is an immortalized endothelial line that retains BBB characteristics in vitro [28]. The cell line was purchased from Cedarlane Labs (Burlington, NC).

\subsubsection{Animals}

All experiments were conducted in accordance with the National Institutes of Health Guide for the Care and Use of Laboratory Animals and were approved by the Institutional Animal Care and Use Committee at West Virginia University. Male wild-type (WT; C57BL6/J; Bar Harbor, ME, Catalog \# 000664) mice were bred in West Virginia University Health Sciences Center vivarium facilities and used for sepsis and stroke experiments at 3-5 months old. Floxed $A / p l\left(A / p f^{f / f f}\right)$ mice on a C57BL/6J genetic background were obtained from Dr. José Luis Millán at the Sanford-Burnham Prebys Medical Discovery Institute and crossed with B6.FVB-Tg(Cdh5-cre)7Mlia/J (VE-Cadherin Cre, Bar Harbor, ME, Catalog \# 006137) mice obtained from Jackson Labs. Creation of $A / p f^{f / f l}$ mice is described in [27] and creation of VECadherin Cre mice is described in [29]. VE-Cadherin Cre and Alpf $\left.\right|^{f / f l}$ mice were crossed to ultimately produce mice with a conditional deletion of $A / p /$ in the endothelium (VE-cKO) and littermate control mice $\left(A / p I^{f / f f}\right)$. For genotyping, DNA was extracted from ear snips using the Purelink Genomic DNA Mini Kit (Invitrogen, Carlsbad, CA, USA), and PCR products were 
amplified by using a Veriti 96-well Thermal Cycler (Applied Biosystems, ThermoFisher Scientific, Waltham, MA) under the following conditions: $94^{\circ} \mathrm{C}$ for $1 \mathrm{~min},\left[\left(94^{\circ} \mathrm{C}\right.\right.$ for $30 \mathrm{sec}$, $60^{\circ} \mathrm{C}$ for $30 \mathrm{sec}, 72^{\circ} \mathrm{C}$ for $\left.\left.45 \mathrm{sec}\right) \times 40 \mathrm{cycles}\right]$, then $72^{\circ} \mathrm{C}$ for $1 \mathrm{~min}$. VE-Cadherin Cre specificity was determined by the presence of a 700 bp product using the following primers; ACRE_F: 5'- GAACCTGATGGACATGTTCAGGGA -3', and ACRE_R: 5'CAGAGTCATCCTTAGCGCCGTAAA -3' [29]. Confirmation of floxed Alp/ sites was determined by the presence of a 263 bp product using the following primer set; Alplflox_F: 5'GTTGCGATGTGTGAAGATGTCCTCG -3', and Alplflox_R: 5'-

CTTGGGCTTGCTGTCGCCAGTAAC -3'. An additional strain was employed. Red fluorescent protein (RFP) B6.Cg-Gt[30]26Sor (Ai9) mice under a genetic C57BL/6J background were obtained from Dr. Eric Tucker in the Department of Neuroscience at West Virginia University; generation of these mice is described in [31]. All mice were group housed in environmentally controlled conditions with a reverse light cycle $\left(12: 12 \mathrm{~h}\right.$ light/dark cycle at $\left.21 \pm 1^{\circ} \mathrm{C}\right)$ and provided food and water ad libitum.

\subsubsection{Cecal Ligation and Puncture (CLP)}

The cecal ligation and puncture (CLP) model of polymicrobial sepsis was employed as previously described [22, 32]. Briefly, C57BL/6J mice were anesthetized by the inhalation of 1$2 \%$ isoflurane and abdominal access was obtained via a midline incision. The cecum was ligated below the ileocecal valve, punctured twice with a $22 \mathrm{G}$ needle through and through, and placed back into the abdominal cavity. The abdominal muscle and skin layer were closed with 60 and $5-0$ sutures (Ethilon, Cornelia, GA) respectively. Sham-operated animals had their cecum isolated and then returned to the peritoneal cavity without being ligated or punctured. One $\mathrm{mL}$ of sterile $0.9 \%$ saline was administered subcutaneously (s.c.) for fluid resuscitation in all experimental groups. Mice used for all experiments were euthanized at seven days post-CLP. 


\subsubsection{Transient Middle Cerebral Artery Occlusion (tMCAO)}

tMCAO surgery was performed under isofluorane anesthesia as previously described [33]. Briefly, male C57BL/6J mice were subjected to 60 min tMCAO using silicon coated sutures (Cat. \#702334, Doccol Corporation, MA) followed by reperfusion. Body temperatures were controlled at $37 \pm 0.5^{\circ} \mathrm{C}$ during occlusion. Occlusion and reperfusion were verified in each animal by a Laser Speckle Imager (Moor Instruments, England). Bupivacaine ( $2 \mathrm{mg} / \mathrm{kg}$, s.c.) was administered to relieve pain after surgery. Mice were followed for seven days post-stroke and euthanized.

\subsubsection{Tissue Collecting and Processing}

Mice were deeply anesthetized with isoflurane and transcardially perfused (Masterflex 7524-10, Cole-Parmer, Vernon Hills, IL) as described previously [22]. Briefly, blood was removed with $0.9 \%$ saline followed by perfusion and fixation with $4 \%$ chilled paraformaldehyde (PFA, Fisher Scientific, Pittsburgh, PA). Perfused brains were post-fixed in 4\% PFA overnight at $4^{\circ} \mathrm{C}$. On the following day, brains were rinsed in $0.01 \mathrm{M}$ phosphate buffered saline (PBS) and incubated sequentially in $15 \%$ and $30 \%$ sucrose in PBS for $24 \mathrm{~h}$ each. Next, brains were coembedded in $15 \%$ gelatin for sectioning. The gelatin block was processed sequentially through $4 \%$ PFA for $24 \mathrm{~h}, 15 \%$ sucrose for $48 \mathrm{~h}$, and $30 \%$ sucrose for $48 \mathrm{~h}$. The block was trimmed and placed in a $-80^{\circ} \mathrm{C}$ freezer for $1 \mathrm{~h}$. Sectioning was performed in the coronal plane at $35 \mu \mathrm{m}$ on a sliding microtome (HM 450, ThermoFisher Scientific).

\subsubsection{Primary Brain Microvascular Endothelial Cell (pBMEC) Culture}

Brain microvascular endothelial cells (BMECs) were cultured from male and female mice as previously described [32]. Briefly, 6-8 weeks old $A / p f^{f / f t}(n=5)$ and VE-cKO $(n=5)$ were perfused with 0.01 M PBS. Cortices were dissected, homogenized, digested in papain and DNase I (Worthington Biochemical Corp, Lakewood, NJ) at $37^{\circ} \mathrm{C}$ for $1 \mathrm{~h}$. The homogenate was 
then centrifuged $(1360 \times \mathrm{g})$ for 10 minutes, followed by myelin removal. The cell pellet was resuspended in endothelial cell growth medium (ECGM: F12 medium with 10\% fetal bovine serum (FBS), endothelial growth supplement, ascorbate $(2.5 \mu \mathrm{g} / \mathrm{ml})$, L-glutamine $(4 \mathrm{mM})$, and heparin $(10 \mu \mathrm{g} / \mathrm{ml})$ ), and plated into four collagen-coated wells (calf skin collagen, SigmaAldrich) of a six-well plate. Cultures were treated with fresh ECGM medium the next day followed by treatment with puromycin hydrochloride $(4 \mu \mathrm{g} / \mathrm{ml})$ with EGCM + FBS for 2.5 days. Cultures reached confluency after 5-7 days and were used for barrier function assays.

\subsubsection{Brain Endothelial Cell Barrier Function Assays}

Barrier function assays were carried out as previously described for hCMEC/D3 cells [22] and in pBMECs [32]. Briefly, hCMEC/D3 cells were seeded onto 3 independent collagencoated 16-well E-Plate PET arrays (ACEA Biosciences, San Diego, CA) at a concentration of 20,000 cells/well and loaded onto an xCelligence RTCA DP system (ACEA Biosciences) enclosed in a cell culture incubator. Once hCMEC/D3 cells reached confluence $\sim 24 \mathrm{~h}$ after seeding, triplicate wells in each array were treated with $200 \mu \mathrm{l}$ of the following: $0.3 \%$ dimethyl sulfoxide (DMSO), tissue-nonspecific alkaline phosphatase inhibitor (TNAPi $100 \mu$ M; Millipore, Temecula, CA), or tumor necrosis factor-alpha and interferon-gamma (TNF- $\alpha$ and IFN-ץ; 10 ng/mL; Sigma-Aldrich, Milwaukee, WI). In a second set of experiments hCMEC/D3 cells in triplicate wells were treated with $200 \mu$ l of the following: $0.3 \%$ DMSO, TNAPi (100 $\mu M)$, fasudil $(10 \mu \mathrm{M}$; Selleckchem, Houston, TX), or TNAPi $(100 \mu \mathrm{M})$ and fasudil $(10 \mu \mathrm{M})$. Barrier function assays were also performed in pBMECs cultures isolated from Alp $f^{f / f f l}$ and VE-cKO mice. Barrier function of pBMEC cultures was recorded both with and without treatments. $A / p f^{f / f f}$ and VE-cKO endothelial cell cultures were treated with vehicle or $10 \mu \mathrm{M}$ fasudil (i.e., Alp $f^{f / f f l}$ untreated, Alp $f^{f / f f l}$ fasudil, VE-cKO untreated, and VE-cKO fasudil). Cell impedance or normalized cell impedance was recorded and analyzed with RTCA Software 2.0 (ACEA Biosciences). Normalized cell impedance is calculated by dividing cell impedance at the normalized time (i.e., when cells are 
treated) by the original cell impedance. Untreated for all experiments refers to cells treated with vehicle (i.e., ECGM medium for pBMECs and endothelial cell growth basal medium-2 (EBM-2) for hCMEC/D3 cells).

\subsubsection{In-Cell Western (ICW) Assay}

The ICW assay was performed using the Odyssey Imaging System (LI-COR Biosciences, Lincoln, NE) as previously described [34]. Briefly, hCMEC/D3 cell cultures were grown in 96-well plates until they reached confluency. Thereafter, hCMEC/D3 cells were treated with vehicle, $0.3 \%$ DMSO, or TNAPi $(100 \mu \mathrm{M})$ for $24 \mathrm{~h}$. The following day, cells were fixed with 4\% PFA then permeabilized with $0.5 \%$ Triton $\mathrm{X}-100$ for $15 \mathrm{~min}$ at room temperature and blocked with LI-COR Odyssey Blocking Solution (LI-COR Biosciences) for $1 \mathrm{~h}$. The cells were then incubated overnight at $4^{\circ} \mathrm{C}$ with primary antibodies. The following primary antibodies were used: ROCK1 (Invitrogen (1:1000), AB_11155392), ROCK2 (Invitrogen (1:1000), AB_11157047), and RhoA (Abcam (1:1000), AB_10675086, Cambridge, MA). The next day, the cells were washed three times with PBS and incubated with the appropriate secondary IgG IRDye ${ }^{\text {TM }}$ 800/680 antibody (1:10,000 dilution, LI-COR Biosciences) at room temperature for $2 \mathrm{~h}$. The 96-well plates were scanned with the Odyssey CLx Infrared Imaging System (LI-COR Biosciences), and the integrated fluorescence intensities representing the protein expression levels were acquired using the Odyssey software (Odyssey Software Version 3.0, LI-COR Biosciences). The relative amount of the protein of interest was obtained by normalizing to total cell number (CellTag700 stain) in all experiments.

\subsubsection{Alkaline Phosphatase Activity Assay Using Homogenized Brain Tissue}

Measurement of alkaline phosphatase activity, quantified as area under the curve [1], from homogenized brain tissue was performed as previously described [32]. Briefly, perfused

mouse brains were homogenized in 500 ul assay buffer (comprised of $1 \mathrm{M} \mathrm{Tris-} \mathrm{HCl}, 1 \mathrm{M} \mathrm{MgCl}$, 
$50 \mathrm{mM} \mathrm{ZnCl}$, and deionized water), centrifuged to remove insoluble material, aliquoted, and frozen at $-80^{\circ} \mathrm{C}$ until analysis. On the day of the assay, brain samples were thawed and diluted 1:10 using assay buffer. Sample wells given assay buffer containing para-nitrophenylphosphate (pNPP; Millipore Sigma, Billerica, MA) for measurement of enzyme activity, were matched to a background control well containing the same buffer as sample wells with the addition of $5 \%$ sodium orthovanadate (BeanTown Chemical, Hudson, NH). This assay was performed in a 384well plate and read kinetically, every 10 mins for 5 hours, on a plate reader at $O_{380}$ using Gen5 Version 2.01.14 software (BioTek Instruments, Inc., Winooski, VT). To compare total enzyme activity over time between genotypes, quantification of AUCs was applied.

\subsubsection{Tissue-Nonspecific Alkaline Phosphatase (TNAP) Enzyme Activity Histology}

Brain tissue section and cell cultures were evaluated for alkaline phosphatase enzyme activity with the BCIP/NBT substrate kit (SK-5400, Vector Laboratories, Burlingame, CA) as previously described $[22,32]$. Tissue sections and cells were rinsed three times in $0.1 \mathrm{M}$ Tris$\mathrm{HCl}(\mathrm{pH}=9.5)$ for $5 \mathrm{~min}$ and incubated in BCIP/NBT staining solution for $4 \mathrm{~h}$ at room temperature. Following incubation, sections were rinsed in $0.01 \mathrm{M}$ PBS and mounted onto microscope slides (Unifrost+, Azer Scientific, Morgantown, PA), air-dried overnight, dehydrated through a standard dehydration series, and cover-slipped with Permount (Fisher Scientific, Pittsburgh, PA).

\subsubsection{Immunohistochemistry}

Brain sections and cell cultures were immunostained using standard immunohistochemistry techniques [22, 32]. Briefly, tissue sections and cells were washed three times, permeabilized, and blocked for 30 min on a shaker. Tissue sections and cells were then incubated for $24 \mathrm{~h}$ with primary antibodies or $1 \mathrm{~h}$ in Alexa 594 phalloidin dye at room temperature, followed by a $2 \mathrm{~h}$ incubation with the appropriate secondary antibody at room 
temperature. The following primary antibodies were used with working dilutions and antibody identification indicated in parentheses: CD31 (RnD Systems (1:500), AB_1026192, Minneapolis, MN), Phalloidin (Invitrogen (1:1000), AB_2315633, Carlsbad, CA), Claudin-5 (Invitrogen (1:200), AB_2806730), and Vimentin (Cell Signaling Technologies (1:500), AB_10695459, Danvers, $\mathrm{MA})$.

\subsubsection{Image Analysis}

Sections were viewed on a Leica DM6B microscope (Leica Camera, Allendale, NJ) and images were captured using Leica LASX software (Leica Microsystems, Buffalo Grove, IL). Cell culture images were captured on the EVOS FL Auto 2 microscope (ThermoFisher Scientific). The cortex, striatum, and hippocampus were identified by referring to the Allen Institute Brain Atlas (http://mouse.brain-map.org). TNAP and/or CD31 images were captured in the cortex, striatum, and hippocampus (40X magnification). 6 fields from 2 sections per animal were collected for the quantification of regional TNAP enzyme activity. CD31 and RFP images were captured in the cortex at 20X magnification. 30 images/cell culture well [35] were used for the quantification of phalloidin, vimentin, and TNAP enzyme activity stains. Images were quantified using the $\mathrm{FIJI} / \mathrm{Image} \mathrm{J}$ version 2.0 software.

\subsubsection{Statistical Analysis}

All experiments were executed to enhance rigor and avoid experimenter bias. Investigators were blinded to the experimental groups for all image analyses. Immunohistochemistry and in-cell western images were analyzed using a one-way analysis of variance (ANOVA) followed by Tukey's multiple comparisons post hoc test. The hCMEC/D3 cell barrier function assay was analyzed using a one-way repeated measure analysis of variance (ANOVA) followed by Tukey's multiple comparisons post hoc test. The pBMECs (i.e., Alp fl/ft versus $\mathrm{VE}-\mathrm{KKO}$ ) barrier function assay was analyzed using a two-way repeated measure 
analysis of variance (ANOVA). Whole brain AP activity assay in Alpfl/fl versus VE-cKO was analyzed using a two-tailed unpaired Student's t-test. Barrier function assay for the fasudil treated pBMECs was analyzed using linear mixed modeling with repeated subject set to cell line and covariance matrix set to compound symmetry. The model effects included the time block (pre-treatment, fasudil treatment, and $48 \mathrm{~h}$ post-fasudil treatment), group ( $A / \mathrm{p} f^{f / f l}$ untreated, $A / p f^{f / f l}$ fasudil, VE-cKO untreated, and VE-cKO fasudil), and time block by group interaction. Comparisons within time blocks were made across groups on the least square means (LS means) with Tukey-Kramer adjustments. All analyses were conducted using SAS 9.4 (SAS software, Cary, NC) and GraphPad Prism 8.1 (GraphPad Software, La Jolla, CA). All p-values and $n$ values are indicated in the figure legends. Results were expressed as means \pm SEM and $p$-values $<0.05$ were considered significant.

\subsection{Results}

\subsubsection{Brain Microvascular TNAP Activity is in Models of Cerebrovascular Dysfunction}

We have previously shown that brain microvascular TNAP activity is decreased at $24 \mathrm{~h}$ post-sepsis, and this decrease in TNAP activity is sustained up to 7 days post-sepsis $[3,22]$. Therefore, we examined whether the decrease in brain microvascular TNAP activity post-sepsis (Fig. 1a) extends to other neuroinflammatory conditions such as stroke. Cortex and striatum were examined because these regions revealed increased microvascular TNAP activity compared to other regions like the hippocampus in mice (Supplementary Fig. 1). Our results showed that following transient middle cerebral artery occlusion, the penumbra in the ipsilateral cortex and striatum exhibited decreased TNAP activity compared to the contralateral cortex and striatum (Fig. 1b). These results are consistent with an earlier study from our group showing that the loss of brain microvascular TNAP activity post-sepsis did not result from the loss of brain microvessels (Supplementary Fig. 2a) [22]. Similarly, CD31 and TNAP activity double-label histology in the penumbra of stroke tissue (striatum shown) revealed a loss of brain 
microvascular TNAP activity that is independent of CD31 positive vessel loss (Supplementary Fig. 2b). The loss of TNAP activity on CD31 positive microvessels after stroke and sepsis led us to speculate that TNAP may play an important role in cerebrovascular inflammation.

\subsubsection{TNAP Inhibition in Brain Microvascular hCMEC/D3 Endothelial Cells Promotes Loss of Barrier Impedance}

Brain microvascular endothelial cells are a key component of cerebral microvessels and play an important role in maintaining barrier integrity [36]. Next, we investigated whether a decrease in TNAP activity in brain endothelial cells promotes barrier dysfunction. Brain microvascular hCMEC/D3 endothelial cells were treated with TNAPi alone, TNF- $\alpha$ and IFN- $\gamma$, or TNF- $\alpha$ and IFN- $\gamma$ combined with TNAPi. Our results showed that treatment of hCMEC/D3 endothelial cells with TNAPi $(p=0.01)$ and TNF- $\alpha$ and IFN-y $(p=0.0005)$ significantly decreased endothelial TNAP activity compared to appropriate controls (Tukey's multiple comparisons test, one-way ANOVA). Furthermore, a combined treatment of TNAPi and TNF- $\alpha$ and IFN- $\gamma$ significantly decreased $(p<0.0001)$ endothelial TNAP activity compared to TNAPi alone (Tukey's multiple comparisons test, one-way ANOVA) (Fig. 2a, b). We then examined whether the loss of TNAP activity in hCMEC/D3 endothelial cells following treatment with TNAPi or TNF- $\alpha$ and IFN-y resulted in a loss of barrier function. Our results showed that both TNAPi $(p$ $<0.0001)$ and TNF- $\alpha$ and IFN-Y $(p<0.0001)$ significantly decreased barrier integrity (impedance) compared to control (Tukey's multiple comparisons test, one-way ANOVA) (Fig. 2c).

\subsubsection{Inhibition of Brain Endothelial TNAP Activity Induces Cytoskeletal Remodeling}

The next studies sought to explore the mechanisms responsible for TNAPi-induced barrier dysfunction shown in Figure 2. Earlier studies by Deracinois et al. with a pan-

phosphatase inhibitor (levamisole) suggested that the TNAP-dependent loss of barrier integrity 
in bovine capillary endothelial cells was associated with cytoskeleton remodeling [37]. However, levamisole is a non-specific inhibitor of TNAP and is capable of decreasing the activity of other phosphatases [26, 38]. Hence, we examined cytoskeletal remodeling by using a specific TNAP inhibitor. To do this, we treated hCMEC/D3 endothelial cells with TNAPi alone, TNF- $\alpha$ and IFN$\mathrm{Y}$, or TNF- $\alpha$ and IFN- $\mathrm{y}$ combined with TNAPi. Thereafter, we immunostained for F-actin using phalloidin and intermediate filaments using vimentin $24 \mathrm{~h}$ following treatment. Our results showed that treatment of hCMEC/D3 endothelial cells with TNAPi alone $(p=0.0008)$ and TNF- $\alpha$ and IFN-y $(p<0.002)$ significantly decreased F-actin fluorescence intensity compared to appropriate controls (Tukey's multiple comparisons test, one-way ANOVA). Furthermore, combined treatment of TNF- $\alpha$ and IFN- $\gamma$ with TNAPi significantly decreased F-actin fluorescence intensity compared to TNAPi alone $(p<0.006)$ or TNF- $\alpha$ and IFN-y $(p=0.0001$; Tukey's multiple comparisons test, one-way ANOVA). Moreover, we observed increased cell detachment (white arrows) in the TNAPi alone, TNF- $\alpha$ and IFN- $\gamma$, and TNF- $\alpha$ and IFN- $\gamma$ combined with TNAPi groups compared to controls (i.e., DMSO or untreated) (Fig. 3a, b). Likewise, intermediate filament (vimentin) fluorescence intensity was significantly decreased in the TNAPi alone $(p=0.03)$ and TNF- $\alpha$ and IFN- $y$ combined with TNAPi $(p=0.008)$ when compared to DMSO control (Tukey's multiple comparisons test, one-way ANOVA). However, treatment with TNF- $\alpha$ and IFN-Y alone did not appear to significantly alter the fluorescence intensity of vimentin (Fig. 3c, d).

\subsubsection{ROCK Protein Expression is Increased Following TNAP Inhibition in Endothelial Cells}

Numerous studies have implicated the Rho/ROCK pathway as a critical mediator involved in endothelial cytoskeletal remodeling [39]. From the results derived in Figure 3, we employed in-cell westerns (ICW) to examine the implications of TNAP inhibition on RhoA, ROCK 1, and ROCK 2 endothelial protein expression. Although RhoA expression increased relative to the appropriate control (DMSO) following treatment with TNAPi; however, this 
increase was not significant (Fig. 4a, b). Next, we examined downstream proteins (i.e., ROCK 1 and ROCK 2) involved in the Rho/ROCK pathway. Our results showed that ROCK $2(p=$ $0.0003)$ and ROCK $1(p=0.02)$ protein expression was significantly increased following TNAPi treatment compared to DMSO control (Tukey's multiple comparisons test, one-way ANOVA) (Fig. 4c-f). Interestingly, DMSO, the vehicle for TNAPi, significantly increased $(p=0.02)$ ROCK1 protein expression relative to untreated endothelial cells (Tukey's multiple comparisons test, one-way ANOVA). This finding was not uncommon since DMSO has been shown to induce slight inflammation [40].

\subsubsection{A ROCK Inhibitor Mitigates Loss of Barrier Impedance Following TNAP Inhibition}

Fasudil is a potent competitive inhibitor of ROCK 1 and ROCK 2 [41]. Therefore, we examined whether fasudil alleviates TNAPi-induced barrier dysfunction. To do this, hCMEC/D3 endothelial cells were treated with TNAPi, fasudil, and TNAPi and fasudil. Following treatment, barrier impedance was measured over a 48-h period. Our results showed that TNAPi treatment significantly decreased $(p<0.0001$, Tukey's multiple comparisons test, repeated one-way ANOVA) barrier impedance compared to DMSO control. A combination treatment of fasudil and TNAPi revealed a significantly improved $(p<0.0001$, Tukey's multiple comparisons test, repeated one-way ANOVA) barrier impedance relative to TNAPi alone (Fig. 5). These results implicate ROCK proteins as downstream mediators of TNAP inhibition.

\subsubsection{Conditional Deletion of TNAP in Primary Brain Microvascular Endothelial Cells Reduces} Barrier Impedance

To further establish an important role for endothelial TNAP in barrier maintenance, we generated a mouse model with a conditional deletion of endothelial $A / p /$ - the gene that encodes TNAP - in endothelial cells [42]. To do this, we bred a Cdh5-Cre driver mouse with the previously published Alp/ floxed mouse [27]. Cdh5 encodes VE-cadherin, a marker of 
endothelial cells. First, we verified that Cre expression was specific for vascular endothelial cells by crossing the Cdh5-Cre mice with Ai9 RFP promoter mice (Supplementary Figure 3). Then, we measured whole brain AP activity in offspring littermates. VE-cKO (endothelial TNAP knockouts) mice revealed a significantly decreased ( $t=5.4, p=0.006$, unpaired t-test) whole brain AP activity compared to Alp $f^{f / f t}$ mice (littermate control) (Fig. 6a). To further demonstrate the loss of TNAP in endothelial cells, we isolated and cultured primary brain microvascular endothelial cells ( $\mathrm{pBMECs}$ ) from Alp $f^{f / f f}$ and VE-cKO mice. Our results revealed an absence of TNAP activity in VE-cKO pBMECs compared to Alp ${ }^{f / t / t}$ pBMECs (Fig. 6b). Additionally, VE-cKO endothelial cells revealed increased cell-cell detachment and loss of junction protein - claudin-5 compared to Alp $f^{f / f t}$ endothelial cells (Supplementary Figure 5). Consequently, we assessed whether the loss of TNAP on VE-cKO pBMECs were comparable to the barrier assay results derived with TNAPi in hCMEC/D3 endothelial cells. Our results revealed a significant loss of barrier impedance $(p<0.0001)$ in VE-cKO pBMECs compared to Alp $f^{f / t / t}$ pBMECs (Tukey's multiple comparisons test, repeated two-way ANOVA) (Fig. 6c).

\subsubsection{Fasudil Rescues Loss of Barrier Impedance in VE-cKO Endothelial Cells}

Next, we assessed whether ROCK inhibition would mitigate the loss of barrier impedance shown in Figure 6c. Barrier impedance data was collected at three timepoints: pretreatment, fasudil treatment, and $48 \mathrm{~h}$ post-fasudil treatment (i.e., drug elimination). Our results shown in Figure 7a (pre-treatment) paralleled the results derived in Figure $\mathbf{6 c}$ as expected (i.e., VE-cKO pBMECs demonstrated a significant loss of barrier impedance $(p=0.0009)$ compared to Alp $f^{f / f t}$ pBMECs (repeated three-way ANOVA). Following treatment with fasudil, barrier impedance in VE-cKO pBMECs significantly improved $(p<0.0001)$ compared to untreated VEcKO pBMECs (Tukey-Kramer comparisons test, linear mixed modeling). Interestingly, the improvement of barrier impedance in fasudil treated VE-cKO pBMECs was comparable $(p=$ 0.89 ) to untreated $A / p f^{f / f t}$ pBMECs (Tukey-Kramer comparisons test, linear mixed modeling) 
(Fig. 7b). $48 \mathrm{~h}$ post-fasudil treatment, barrier impedance in fasudil treated VE-cKO pBMECs declined and became comparable $(p=0.52)$ to untreated VE-cKO pBMECs (Tukey-Kramer comparisons test, linear mixed modeling) (Fig. 7c).

\subsection{Discussion}

In the present study, we sought to elucidate the mechanistic role of an AP isozyme, TNAP, in brain microvascular endothelial cells. We have shown previously that microvascular TNAP activity is decreased as early as $24 \mathrm{~h}$ post-sepsis and sustained up to 7 days post-sepsis in the brains of mice $[3,22]$. In addition, we showed that the decrease in TNAP activity in brain microvessels extrapolates to other acute inflammatory conditions such as stroke. The observation of a loss microvascular TNAP activity in late (7 days) sepsis and ischemic stroke highlights a putative role for TNAP at the brain's vasculature. Our results demonstrate an important mechanistic role for endothelial TNAP in maintaining barrier integrity (measured as impedance) by preventing the ROCK-mediated disruption of the cytoskeleton. Of note, it

remains unclear how TNAP's action on ROCK proteins converge into the well-known function of TNAP involving the dephosphorylation of nucleotides, LPS, DAMPs, and PAMPs [15-17]. Inflammatory molecules such as LPS have been shown in other models to increase ROCK protein expression and cause endothelial barrier integrity loss [43-45]; hence, it is likely that the action of LPS, DAMPs, and PAMPs are upstream relative to the novel mechanistic findings shown in this study. Future studies will address the convergence of these pathways.

Circulating levels of pro-inflammatory cytokines such as TNF- $\alpha$ and IFN- $\gamma$ have been shown to be increased in neuroinflammatory conditions like sepsis and stroke [21, 46-48]. Our laboratory has previously shown that there is a sustained increase in pro-inflammatory cytokines up to 7 days post-sepsis, and this increase is coupled to a loss of TNAP activity in brain microvessels [22]; therefore, we examined whether treatment with TNF- $\alpha$ and IFN- $\gamma$ decreased 
endothelial TNAP activity similar to TNAPi. Our results showed that treatment of the brain microvascular D3 endothelial cells with TNF- $\alpha$ and IFN- $\gamma$ sufficiently decreased TNAP activity similarly to TNAPi treatment alone. This finding demonstrates that pro-inflammatory cytokines may, in part, be responsible for the initial loss of brain microvascular TNAP activity seen in sepsis and stroke. Furthermore, we showed that the decrease in endothelial TNAP activity or the conditional loss of TNAP worsened endothelial cell barrier impedance. Owing to these findings, we propose that impaired BBB function seen in sepsis and stroke pathogenesis originates from endothelial cell damage, and the loss of endothelial TNAP activity contributes to BBB dysfunction.

The overall findings from our pharmacological and genetic studies have identified a novel molecular mechanism through which brain endothelial cell TNAP regulates BBB impedance. Importantly, our results substantially extend the findings from a previous study which showed that treatment of bovine capillary endothelial cells with a pan-AP inhibitor, i.e. levamisole, induced the retraction of endothelial cells [37]. Endothelial cell retraction and detachment are prototypical indicators of cytoskeletal reorganization and have been shown to accompany BBB dysfunction and increase cellular permeability $[49,50]$. We observed that treatment of BMECs with TNF- $\alpha$ and IFN-y combined with inhibition of TNAP enzymatic activity disrupted the actin cytoskeleton. More importantly, the reorganization of the BMEC actin cytoskeleton was accompanied by increased cell detachment between adjacent endothelial cells, which represents reduced cell-cell junctional protein contact and adhesion. Junctional proteins such as claudin-5 are connected to the actin cytoskeleton via scaffold proteins and have been shown to play an important role in maintaining paracellular barrier permeability [51]. Therefore, it becomes plausible that the observed TNAPi-induced loss of endothelial barrier impedance shown in this study originates from the inability of brain endothelial cells to form proper cell-cell tight junctions. Therefore, we propose that the observed TNAPi-induced loss of endothelial barrier impedance shown in this study originates from the inability of brain 
endothelial cells to form proper cell-cell adhesion. This is supported by a previous in vivo study from our laboratory which showed that the junctional protein claudin-5 is decreased in the brains of septic mice treated intraperitoneally with an in vivo TNAP inhibitor, SBI-425, compared to vehicle treated septic mice [22]. In addition, morphological analyses of endothelial TNAP conditional knockout cell cultures with the AP activity stain revealed endothelial retraction and cellular detachment similar to TNAPi treated microvascular D3 endothelial cells (data not shown).

Intermediate filaments have been shown to directly and indirectly interact with actin [52, 53]. This interaction is demonstrated by studies which showed that actin disruption affected intermediate filament sub-localization networks in cells [54-56]. We observed that the disruption of actin following TNAP enzyme inhibition disrupted the intermediate filament. Vimentin is one of the major intermediate filaments shown to provide a structural support for cells [57].

Interestingly, we only observed diminished vimentin fluorescence intensity following inhibition of TNAP activity, but not with TNF- $\alpha$ and IFN- $\gamma$ treatment. In contrast, other studies that employed different cell types, human umbilical vein endothelial cells (HUVECs) or astrocytes, have demonstrated that TNF- $\alpha$ treatment increased vimentin protein expression $[58,59]$. We suggest that the properties of individual cell types, the dosage used, and use of TNF- $\alpha$ alone instead of TNF- $\alpha$ and IFN-Y are responsible for this discrepancy. Taken together, our results suggest that the sustained loss of microvascular TNAP activity induced by the presence of pro-inflammatory cytokines may be detrimental to the actin cytoskeleton and intermediate filaments. However, it remains unclear whether the loss of actin initiates vimentin disruption following TNAPi treatment or vice versa.

ROCK protein expression has been shown to play a role in regulating cytoskeletal proteins such as vimentin and actin by initiating F-actin contraction/retraction and cellular detachment $[39,41,49]$. Upstream of ROCK is the RhoA protein, one of many proteins that drives the activation of ROCK, and the Rho-ROCK pathway has been shown to play an 
important role in endothelial cell function Based on our cytoskeletal findings, we addressed whether the Rho/ROCK pathway was implicated in TNAP signaling. Our results showed that ROCK (1/2) protein expression was significantly increased following TNAPi treatment, along with a trending decrease in RhoA. protein levels. This finding indicates that TNAP-mediated signaling mechanism which suppresses ROCK activation is likely mediated by at least one separate pathway in addition to the canonical RhoA pathway. We speculate that the drastic effect of TNAP inhibition on ROCK 2 compared to ROCK 1 protein expression arises from the increased expression of ROCK 2 in brain tissue compared to ROCK 1 [41]. Furthermore, it is also likely that ROCK 1 and ROCK 2 may play independent effector roles downstream. For example, Shi et el., showed that ROCK 1 regulates the actin cytoskeleton through myosin light chain 2 (MLC2) phosphorylation while ROCK 2 regulates the actin cytoskeleton through cofilin phosphorylation in mouse embryonic fibroblast (MEF) cells [60]. Finally, we utilized a ROCK1/2 inhibitor [61] to further demonstrate the involvement of ROCK proteins in a novel TNAP signaling pathway in both D3 cells and pBMECs. These results contribute a novel mechanism in support of the neuroprotective and anti-inflammatory benefit of fasudil treatment established in preclinical models of sepsis and stroke [61-64].

BBB dysfunction is a common feature in many neuroinflammatory disorders [5]. Diminished BBB function is characterized by increased loss of junctional proteins, increased paracellular permeability of molecules and immune cells into the brain parenchyma, and endothelial cell transcytosis of immune cells into the parenchyma [3]. To our knowledge, this is the first study to describe the novel role played by endothelial TNAP in maintaining paracellular barrier impedance since our barrier assays can only assess this parameter. Our results suggest that endothelial TNAP exerts an inhibitory function on the activity of endothelial ROCK proteins in health and this function is dysregulated during acute injury as suggested by our septic and stroke studies. We propose a working model (Fig. 8) in which TNAP inhibition or loss prevents the contraction/retraction of endothelial cells and junctional protein disruption. During 
inflammation and/or acute injury, the rapid increase in pro-inflammatory cytokines leads to a reduction in endothelial TNAP activity on microvessels. The sustained loss of TNAP activity leads to cytoskeletal remodeling indicated by endothelial contraction and junctional protein detachment, which then permits the paracellular infiltration of pro-inflammatory molecules and immune cells that promote astrogliosis and microglial activation.

Despite the current advances discussed in this study, some limitations exist. Since the experiments were carried out using in vitro and ex vivo methods, the contribution of astrocytes or pericytes is not clear and will be addressed in future studies. Since D3 cells were generated from a female donor and mixed sex pBMECs were used in the ex vivo experiments, we were unable to delineate sex-specific pathways. Future studies will address the role of sex on brain endothelial TNAP function. Furthermore, the barrier assay used in this study was unable to delineate how endothelial TNAP may affect transcellular permeability or alter the physiological and metabolic functions of brain endothelial cells. These mechanisms will also be addressed in future studies. In summary, our results support a novel role for TNAP signaling in maintaining BMEC barrier integrity. Therapeutic strategies that modulate the endothelial TNAP activity may be beneficial for the treatment of BBB dysfunction or temporal - targeted drug delivery for various neurological disorders such as brain tumors, ischemic stroke, or Alzheimer's disease.

\section{Author contributions}

D.C.N and C.M.B designed the studies. D.C.N. and W.W. performed all cell culture experiments. D.C.N. and N.B. performed image analyses. A.L.B. generated and genotyped $A / p f^{f / f f l}$ and VE-cKO animals needed for experiments. D.C.N. and C.L.L. performed statistical analyses of data and generation of graphs. D.C.N. and C.M.B. wrote the manuscript. JL.M. provided $A / p f^{f / f f l}$ mice. All authors read and revised the final manuscript. 
Acknowledgement: The authors gratefully thank the West Virginia University Office of Laboratory Animal Resources and the West Virginia University Experimental Stroke Core for their assistance with stroke surgeries. Funding for this work was supported by the NIH T32 AG052375 (D.C.N, A.L.B), K01 NS081014 (C.M.B), West Virginia Clinical and Translational Science Institute (U54 GM104942), and the West Virginia University Stroke CoBRE (P20 GM109098). 
Figures

Figure 1
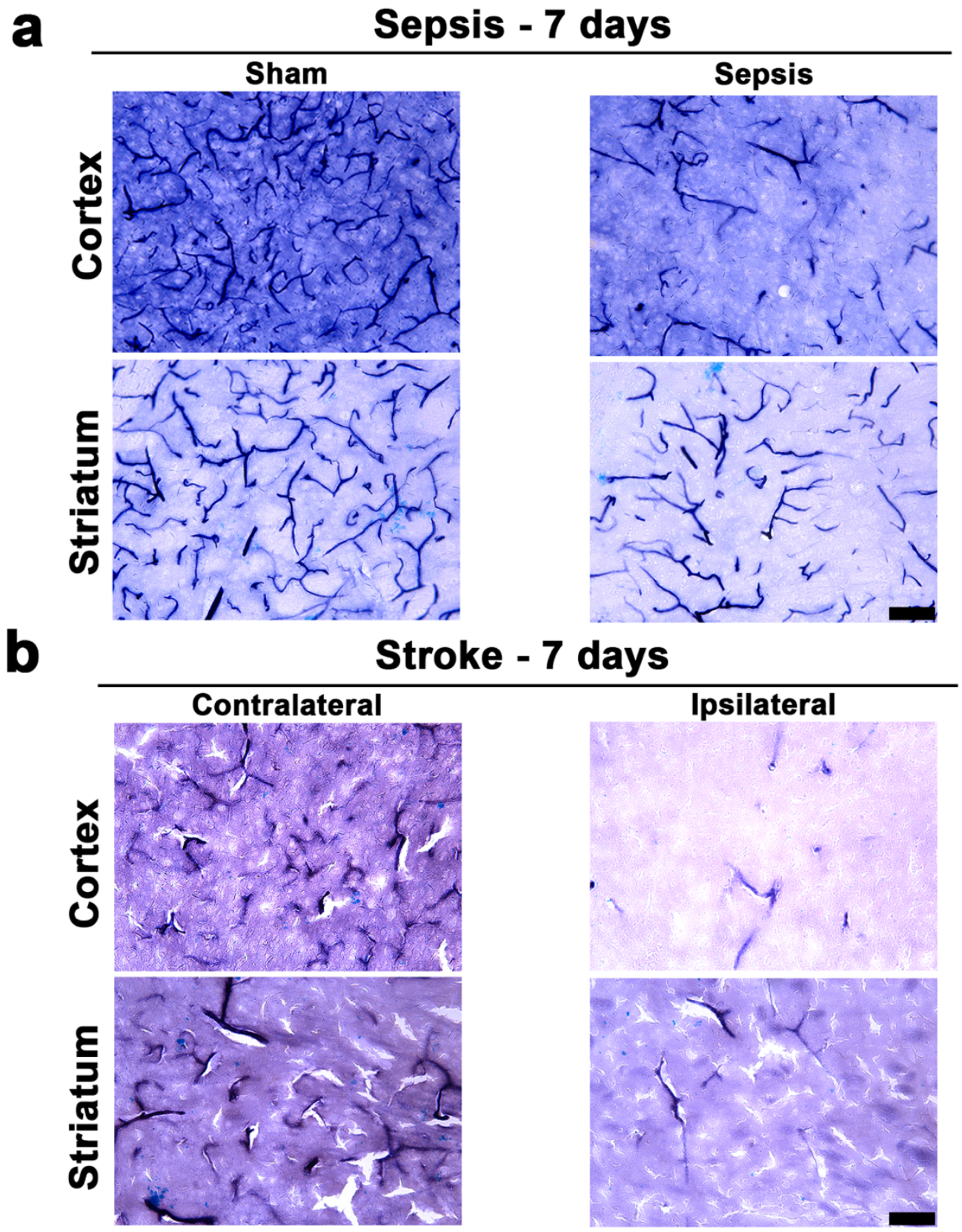
Figure 2

a

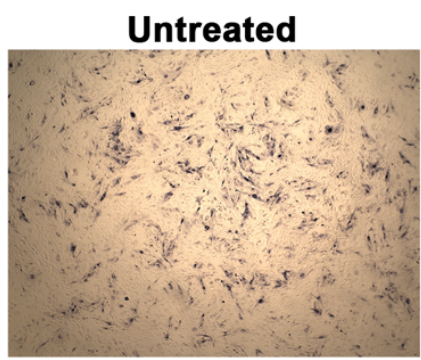

TNAPi

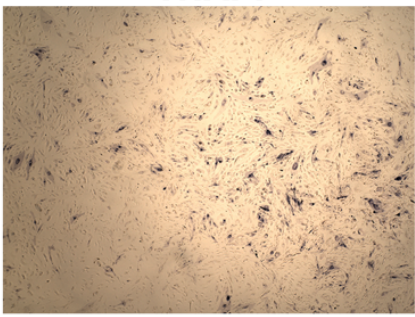

TNAPi + TNF- $\alpha+I F N-\gamma$
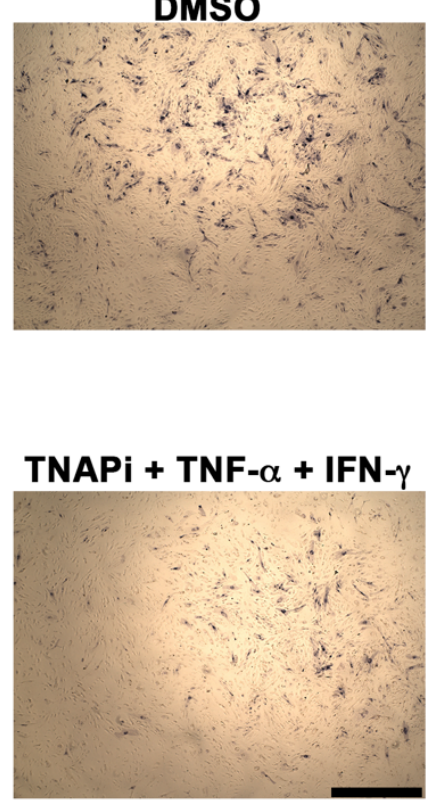

TNF- $\alpha+$ IFN- $-\gamma$

b

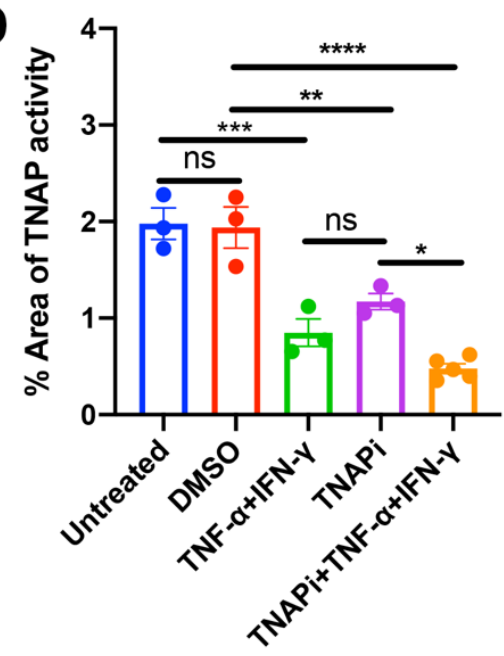

Treatment

C

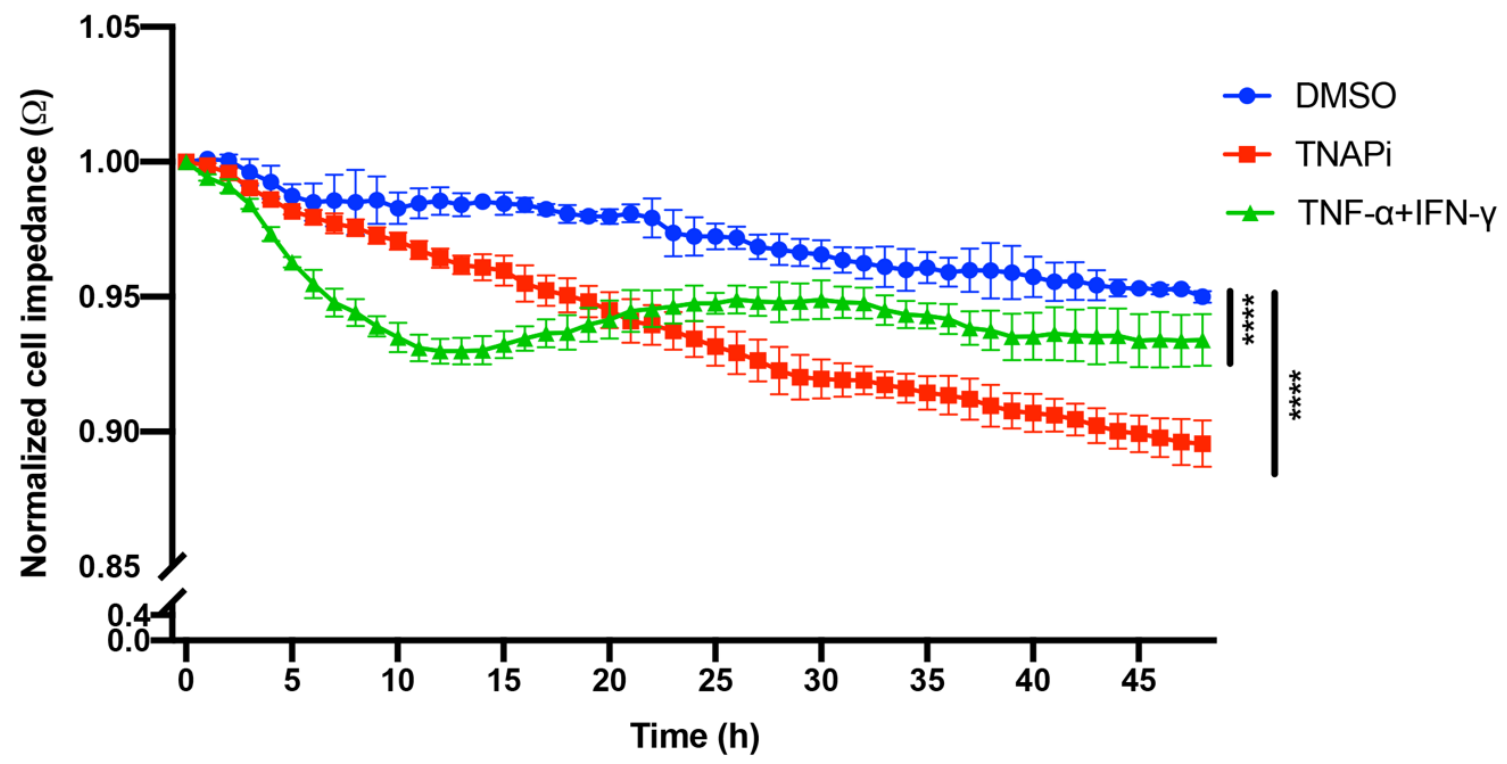


Figure 3

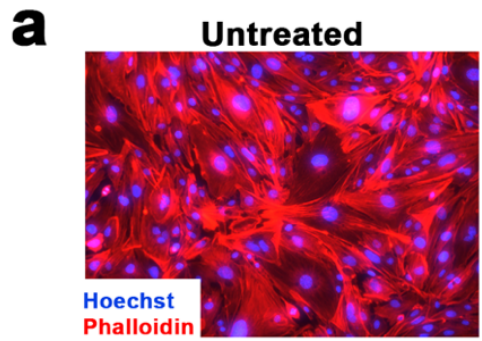

TNAPi

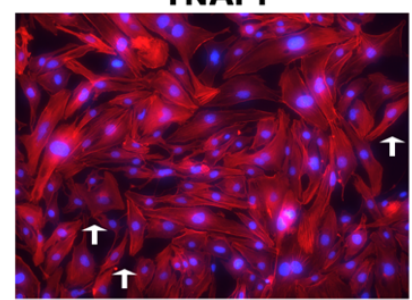

C

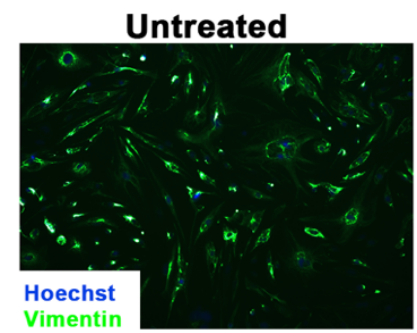

TNAPi

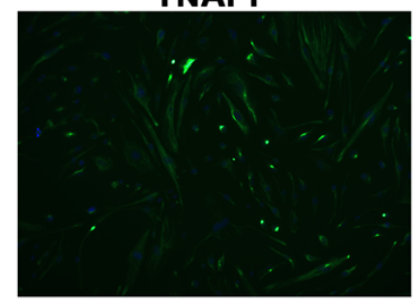

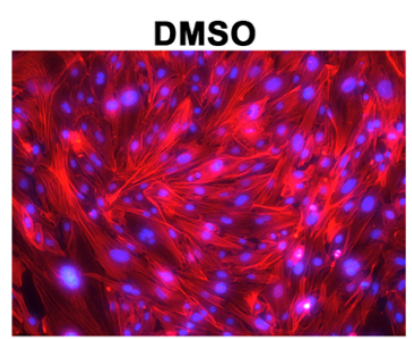

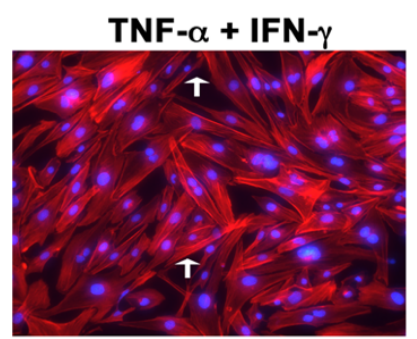

\section{b}

TNAPi + TNF- $\alpha+$ IFN- $-\gamma$
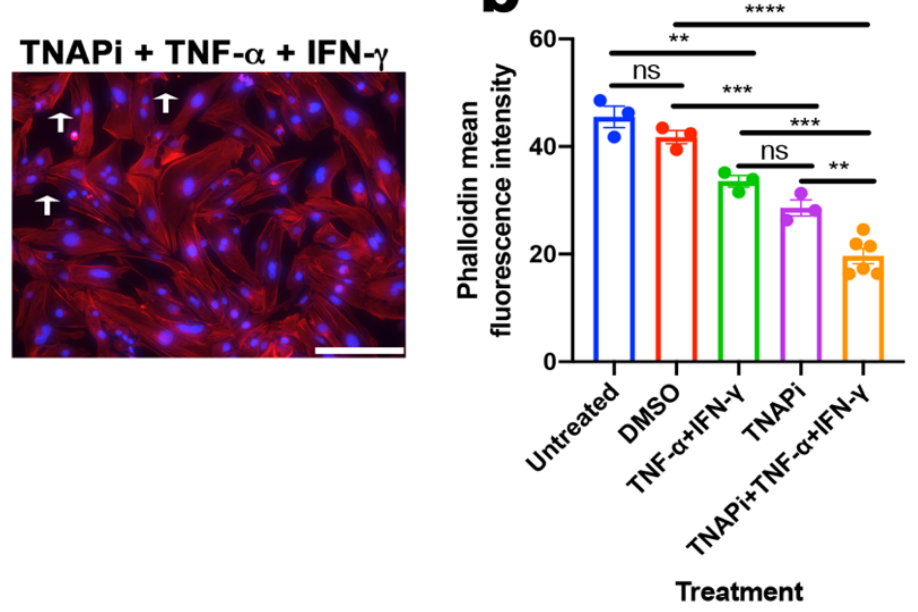

DMSO

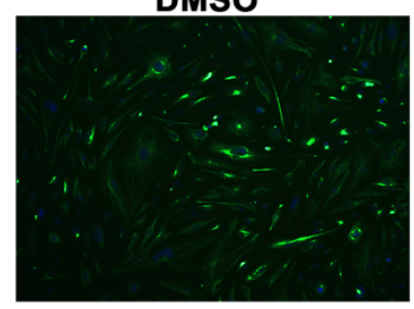

TNF- $\alpha+$ IFN- $-\gamma$

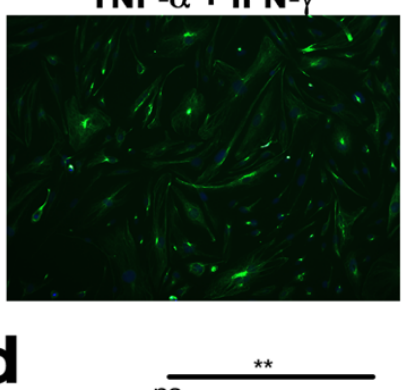

TNAPi + TNF- $\alpha+$ IFN- $-\gamma$

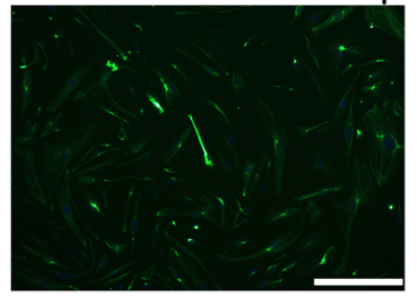

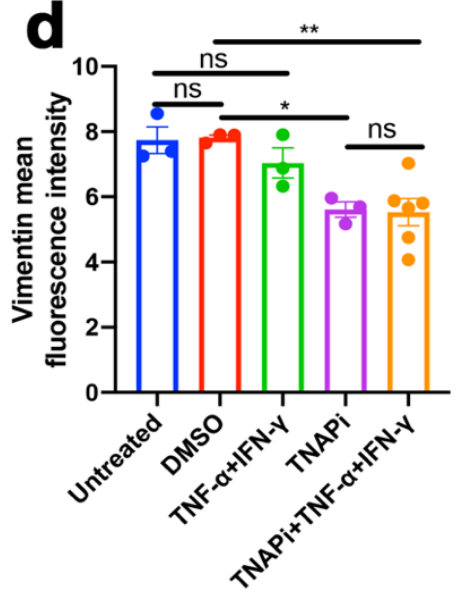

Treatment 
Figure 4

a RhoA

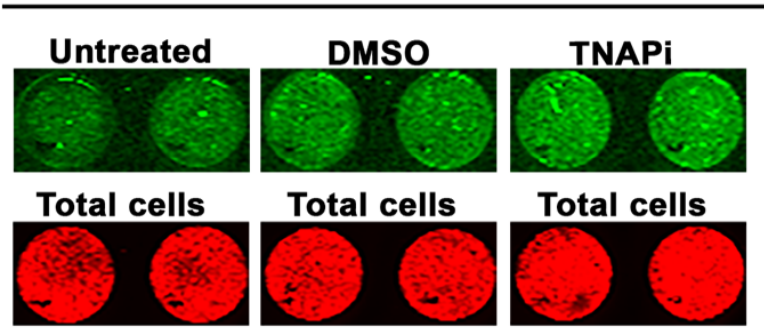

b

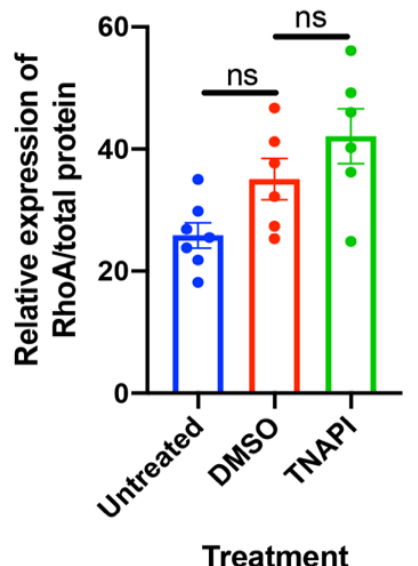

e ROCK1

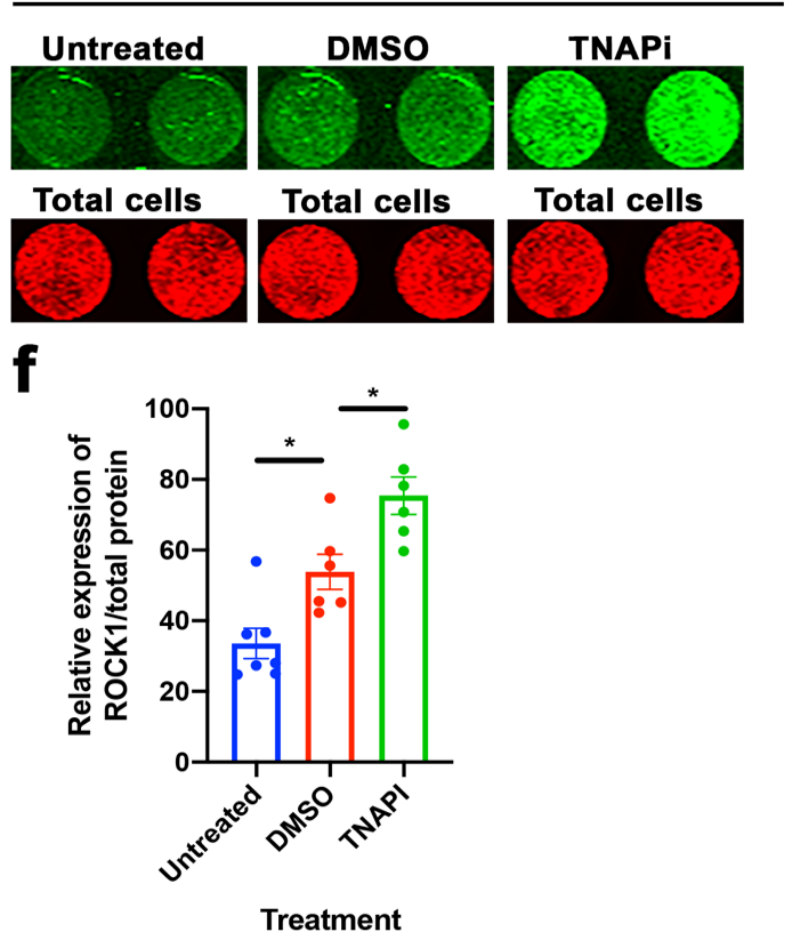

c ROCK2
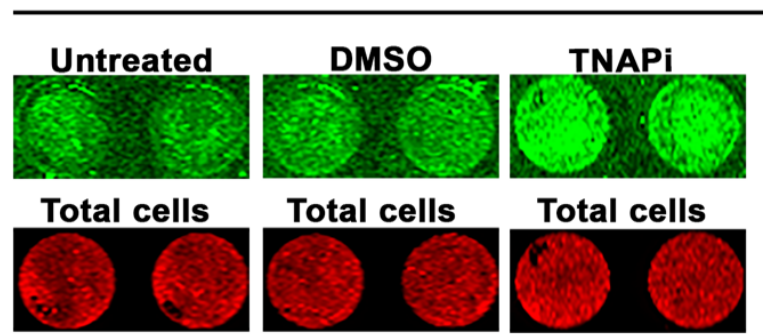

d

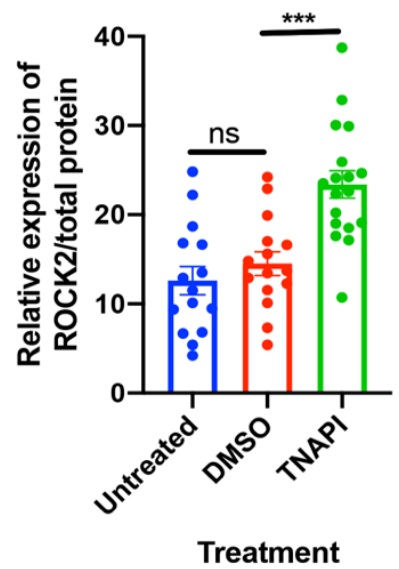


Figure 5

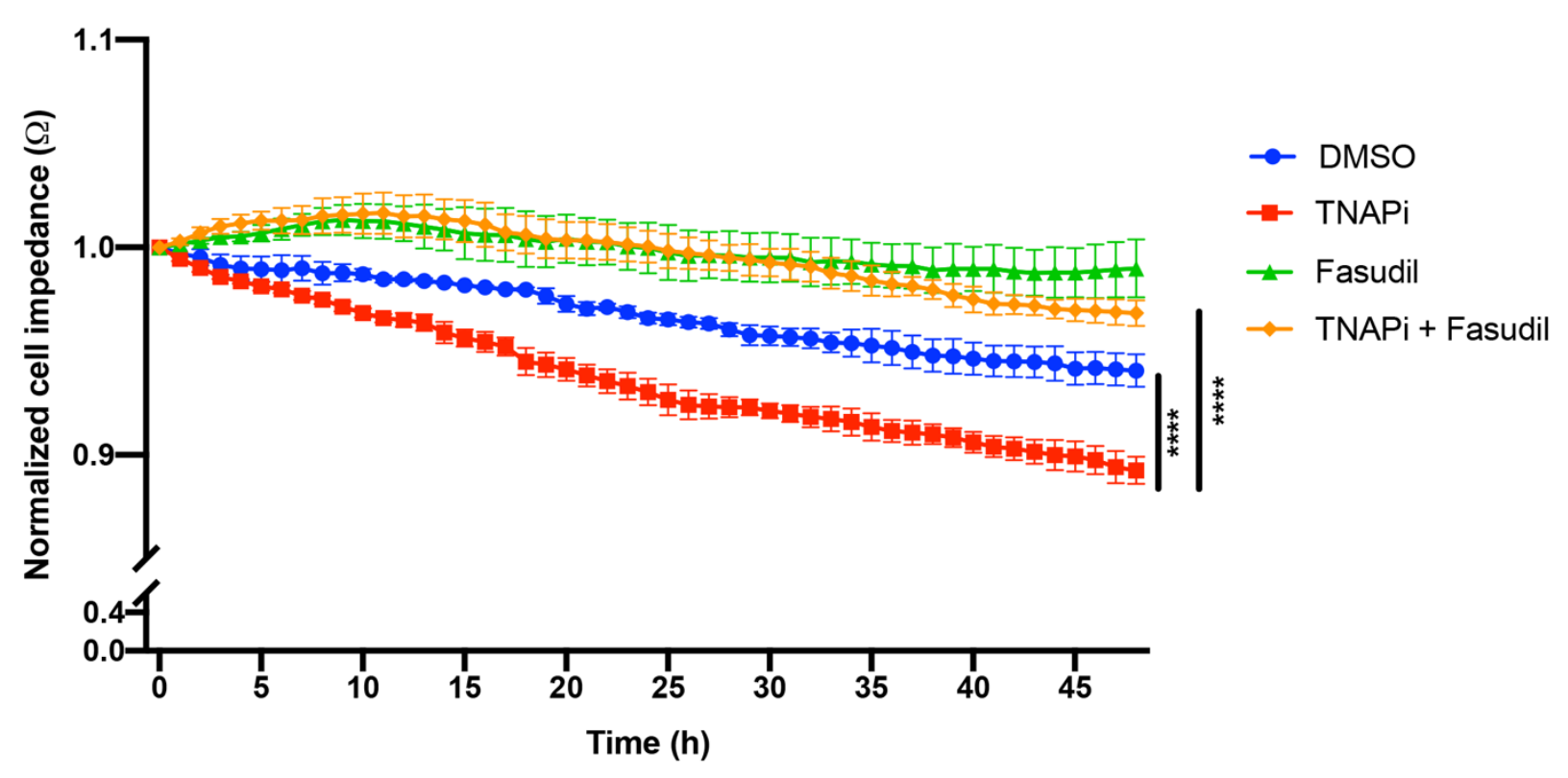


Figure 6

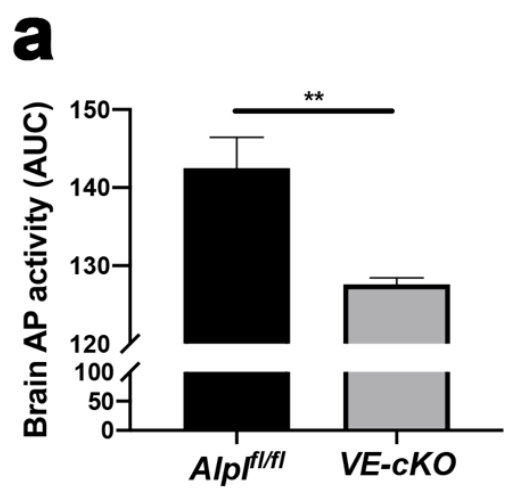

b
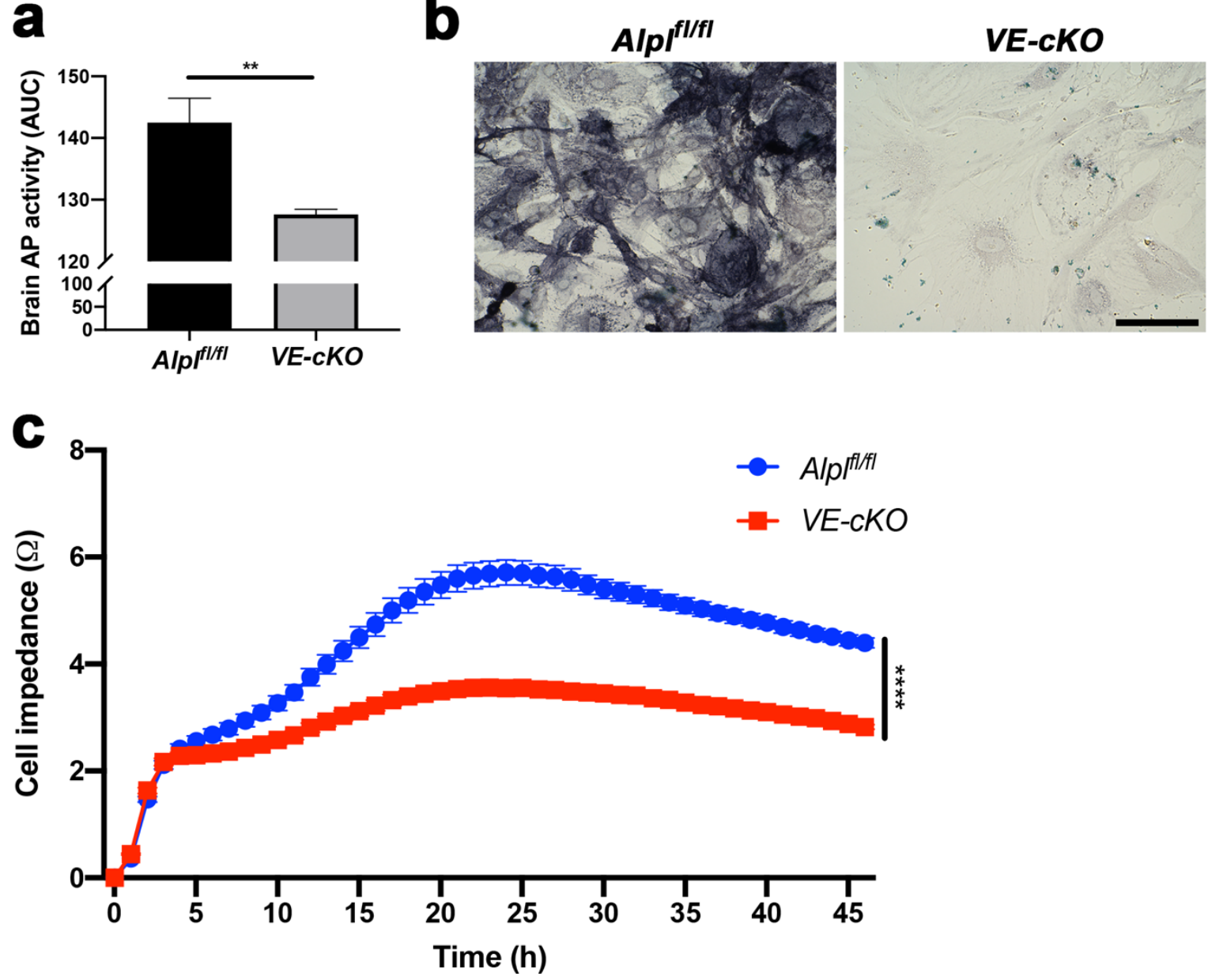
Figure 7

a

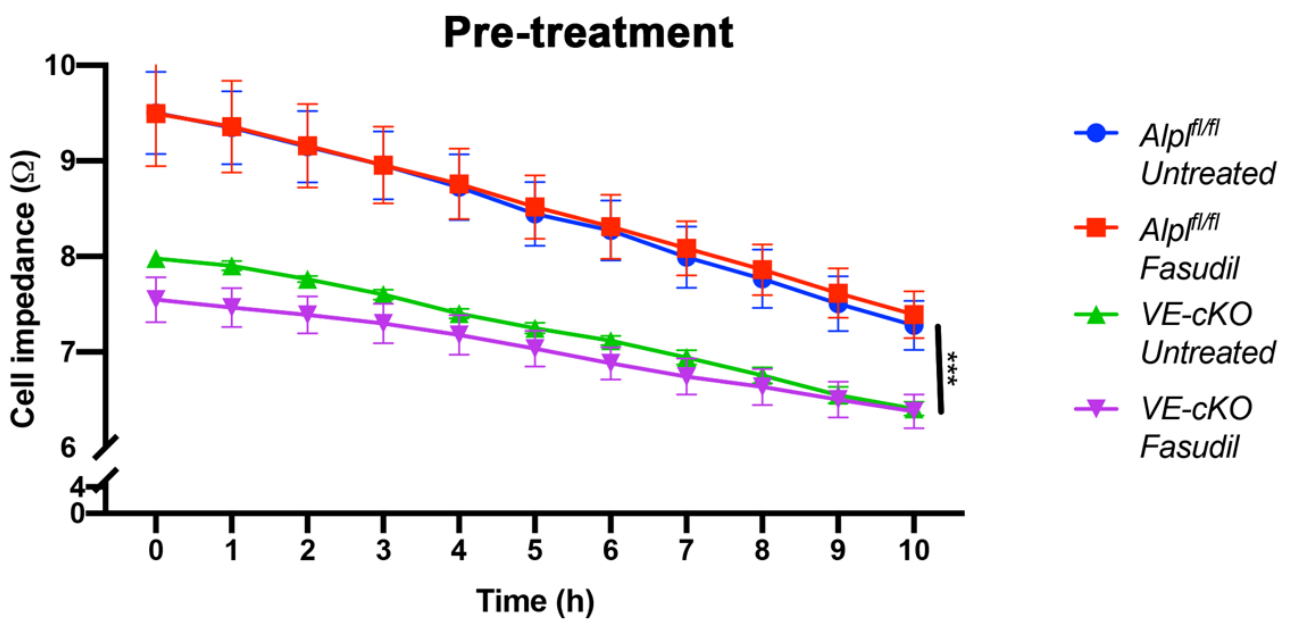

b

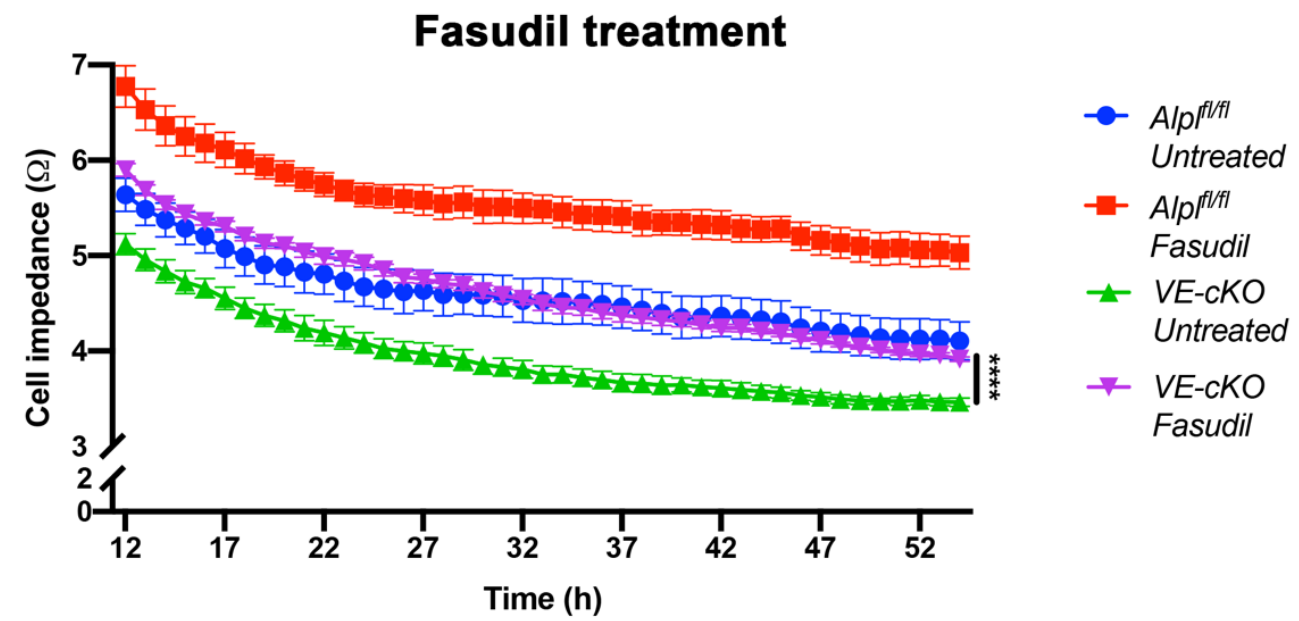

C

48 h Post-fasudil treatment

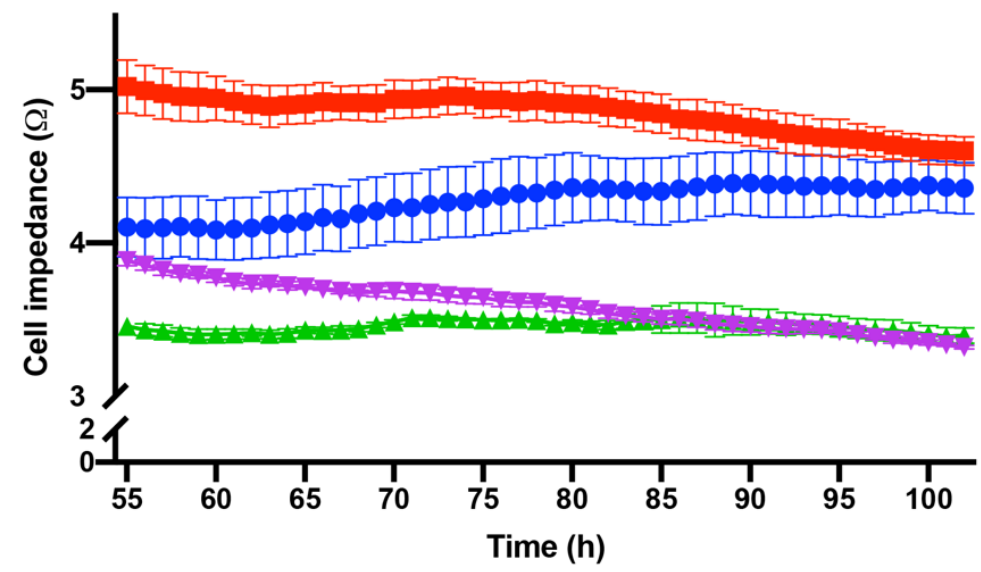

$$
\begin{aligned}
- & \text { Alpflffl } \\
& \text { Untreated } \\
- & \text { Alpflffl } \\
& \text { Fasudil } \\
- & \text { VE-cKO } \\
& \text { Untreated } \\
- & \text { VE-cKO } \\
& \text { Fasudil }
\end{aligned}
$$




\section{Figure Legends}

Figure 1: TNAP activity is decreased in sepsis and stroke (AD). (A-B) Representative images showed that TNAP activity in the cortex and striatum is decreased in sepsis ( 7 days post-sepsis) and stroke (7 days post-stroke) compared to appropriate controls. Images taken at 20X magnification and scale bar $=75 \mu \mathrm{m}$.

Figure 2: Inhibition of brain hCMEC/D3 endothelial TNAP promotes barrier dysfunction. (A, B) TNAPi ( $p=0.01$, Tukey's multiple comparisons test, one-way ANOVA) and TNF- $\alpha$ and IFN- $\gamma$ ( $p=0.0005$, Tukey's multiple comparisons test, one-way ANOVA) significantly decreased brain endothelial TNAP activity compared to appropriate controls (DMSO or untreated). Moreover, combined treatment of TNAPi, TNF- $\alpha$ and IFN- $\gamma$ significantly decreased $(p<0.0001$, Tukey's multiple comparisons test, one-way ANOVA) brain endothelial TNAP activity compared to TNAPi alone. (C) TNAPi $(p<0.0001$, Tukey's multiple comparisons test, repeated one-way ANOVA) and TNF- $\alpha$ and IFN- $\gamma(p<0.0001$, Tukey's multiple comparisons test, repeated oneway ANOVA) significantly decreased barrier integrity (impedance) compared to DMSO control. * indicates $p<0.05,{ }^{* *} p<0.01{ }^{* * *} p<0.001$, and ${ }^{* * * *} p<0.0001$, and is considered significant. All data presented as mean \pm SEM. Images taken at 10X magnification and scale bar $=1000 \mu \mathrm{m} . \mathrm{n}$ $=3-5$ wells/treatment group, ns $=$ not significant.

Figure 3: TNAPi induces cytoskeletal remodeling in hCMEC/D3 brain endothelial cells. (A, B) TNAPi ( $p=0.0008$, Tukey's multiple comparisons test, one-way ANOVA) and TNF- $\alpha$ and IFN- $\gamma(p<0.002$, Tukey's multiple comparisons test, one-way ANOVA) significantly decreased phalloidin (F-actin) fluorescence intensity compared to appropriate controls (DMSO or untreated). Furthermore, combined treatment of TNAPi, TNF- $\alpha$ and IFN- $\gamma$ significantly exacerbated the decrease in phalloidin fluorescence intensity compared to TNAPi $(p<0.006$, Tukey's multiple comparisons test, one-way ANOVA) or TNF- $\alpha$ and IFN- $\gamma$ alone $(p=0.0001$, Tukey's multiple comparisons test, one-way ANOVA). Increased endothelial cell detachment (gaps, white arrows) accompanied the decrease in phalloidin fluorescence intensity. (C, D) TNAPi ( $p=0.03$, Tukey's multiple comparisons test, one-way ANOVA) and combined treatment of TNAPi, TNF- $\alpha$ and IFN- $\gamma(p=0.008$, Tukey's multiple comparisons test, one-way ANOVA) significantly decreased vimentin fluorescence intensity compared to DMSO control. Interestingly, TNF- $\alpha$ and IFN- $\gamma$ alone did not alter vimentin fluorescence intensity. * indicates $p<$ $0.05,{ }^{* *} p<0.01{ }^{* * *} p<0.001$, and ${ }^{* * * *} p<0.0001$, and is considered significant. All data presented as mean \pm SEM. Images taken at $20 X$ magnification and scale bar $=200 \mu \mathrm{m} . \mathrm{n}=3-6$ wells/treatment group, ns = not significant.

Figure 4: ROCK isoforms are increased following TNAPi treatment in hCMEC/D3 brain endothelial cells. (A, B) Rho protein was increased following treatment with TNAPi; however, this increase was not significant $(p=0.34$, Tukey's multiple comparisons test, one-way ANOVA). (C-F) Treatment with TNAPi increased ROCK 2 ( $p=0.0003$, Tukey's multiple comparisons test, one-way ANOVA) and ROCK 1 ( $p=0.02$, Tukey's multiple comparisons test, one-way ANOVA) protein expression compared to DMSO control. Fluorescence signal was normalized to total cell number for each respective well. ${ }^{*}$ indicates $p<0.05$ and ${ }^{* * *} p<0.001$, and is considered significant. All data presented as mean \pm SEM. Average $n=10$ wells/treatment group, ns = not significant 
Figure 5: Fasudil mitigates TNAPi-induced barrier dysfunction in hCMEC/D3 brain endothelial cells. TNAPi alone significantly decreased $(p<0.0001$, Tukey's multiple comparisons test, repeated one-way ANOVA) barrier impedance compared to DMSO control. Combined treatment of TNAPi and fasudil significantly $(p<0.0001$, Tukey's multiple comparisons test, repeated one-way ANOVA) rescues TNAPi-induced barrier dysfunction. ${ }^{* * * *}$ indicates $p<0.001$, and is considered significant. All data presented as mean \pm SEM. $n=3$ wells/treatment group.

Figure 6: Conditional loss of endothelial TNAP worsens barrier impedance. $(A)$ Whole brain AP activity is significantly increased $\left(p=0.006\right.$, unpaired t-test) in $A / p f^{f / f l}(n=2$, littermate control) mice compared to VE-cKO ( $n=4$, endothelial TNAP knockout) pBMECs. (B) VE-cKO pBMECs were absent of TNAP activity stain (purple) compared to Alp $f^{l / f l}$ pBMECs. (C) Barrier impedance in VE-cKO pBMECs was significantly decreased $(p<0.0001$, Tukey's multiple comparisons test, repeated two-way ANOVA) compared to Alp $f^{f / f 1}$ pBMECs. ${ }^{* *}$ indicates $p<0.01$ and ${ }^{* * * *} p<0.0001$, and is considered significant. All data presented as mean \pm SEM. Images taken at $40 \mathrm{X}$ magnification and scale bar $=100 \mu \mathrm{m} . \mathrm{n}=4$ wells/treatment group.

Figure 7: Fasudil mitigates barrier impedance loss in VE-cKO pBMECs. (A) Barrier impedance was significantly decreased ( $p=0.0009$, repeated three-way ANOVA) in VE-cKO pBMECs compared to Alp $f^{f / f l}$ pBMECs before fasudil treatment. (B) Fasudil treatment significantly mitigated ( $p<0.0001$, Tukey-Kramer comparisons test, linear mixed modeling) loss of barrier impedance in fasudil treated VE-cKO pBMECs compared untreated VE-cKO pBMECs. (C) $48 \mathrm{~h}$ post-fasudil treatment (i.e., drug elimination) barrier impedance in fasudil treated VEcKO pBMECs was comparable $(p=0.52$, Tukey-Kramer comparisons test, linear mixed modeling) to untreated VE-cKO pBMECs. ${ }^{* * *}$ indicates $p<0.001$, and ${ }^{* * * *} p<0.0001$, and is considered significant. All data presented as mean \pm SEM. $n=3$ wells/treatment group. 


\section{Supplementary Figures}

\section{Supplementary Figure 1}

a

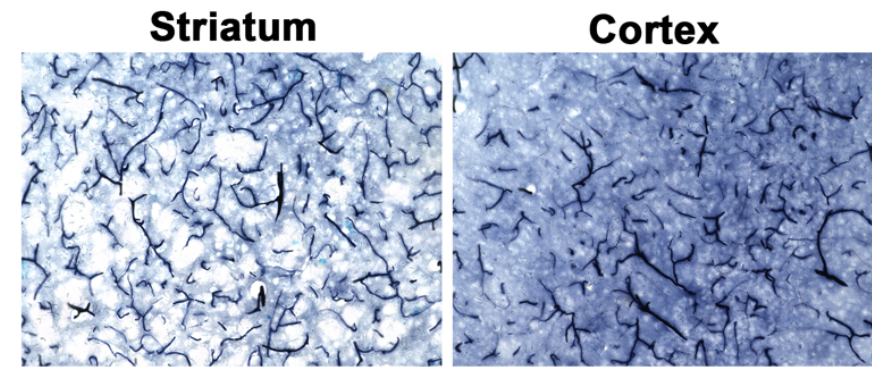

Hippocampus

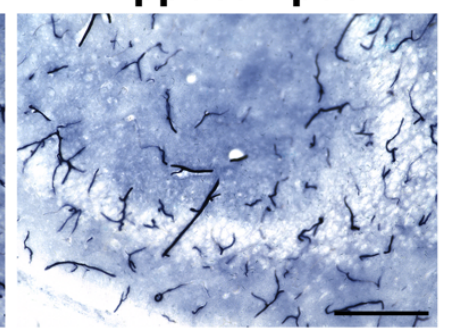

b

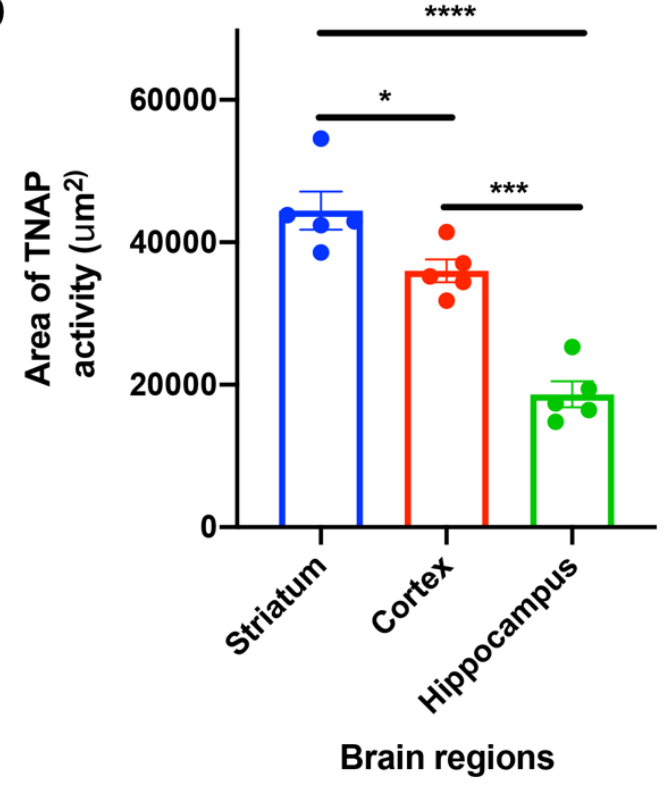


Supplementary Figure 2

a

Sepsis - 7 days
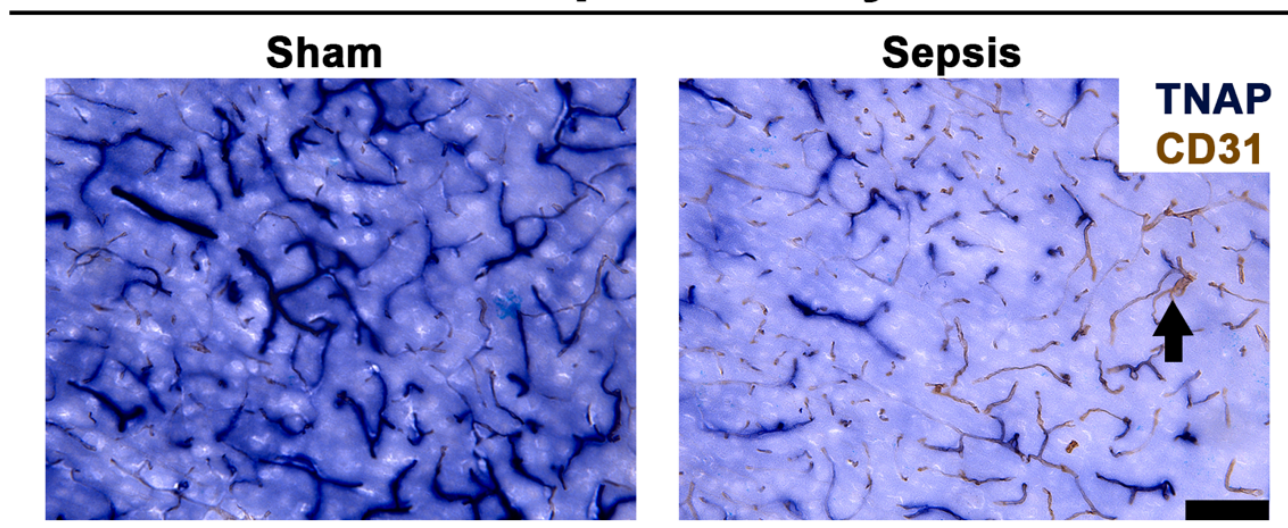

b

Stroke - 7 days
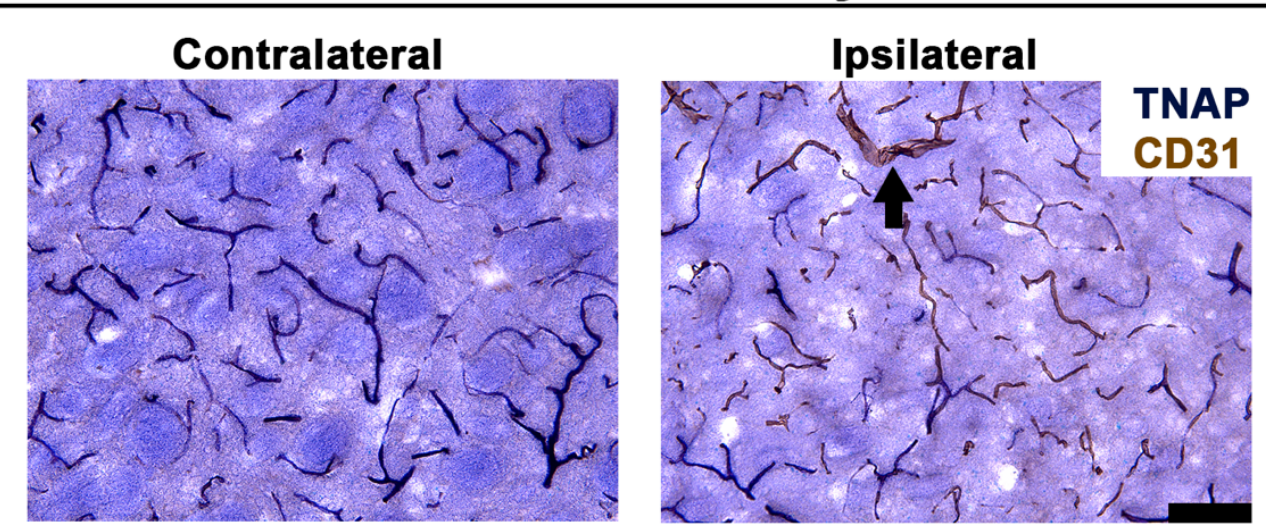
Supplementary Figure 3

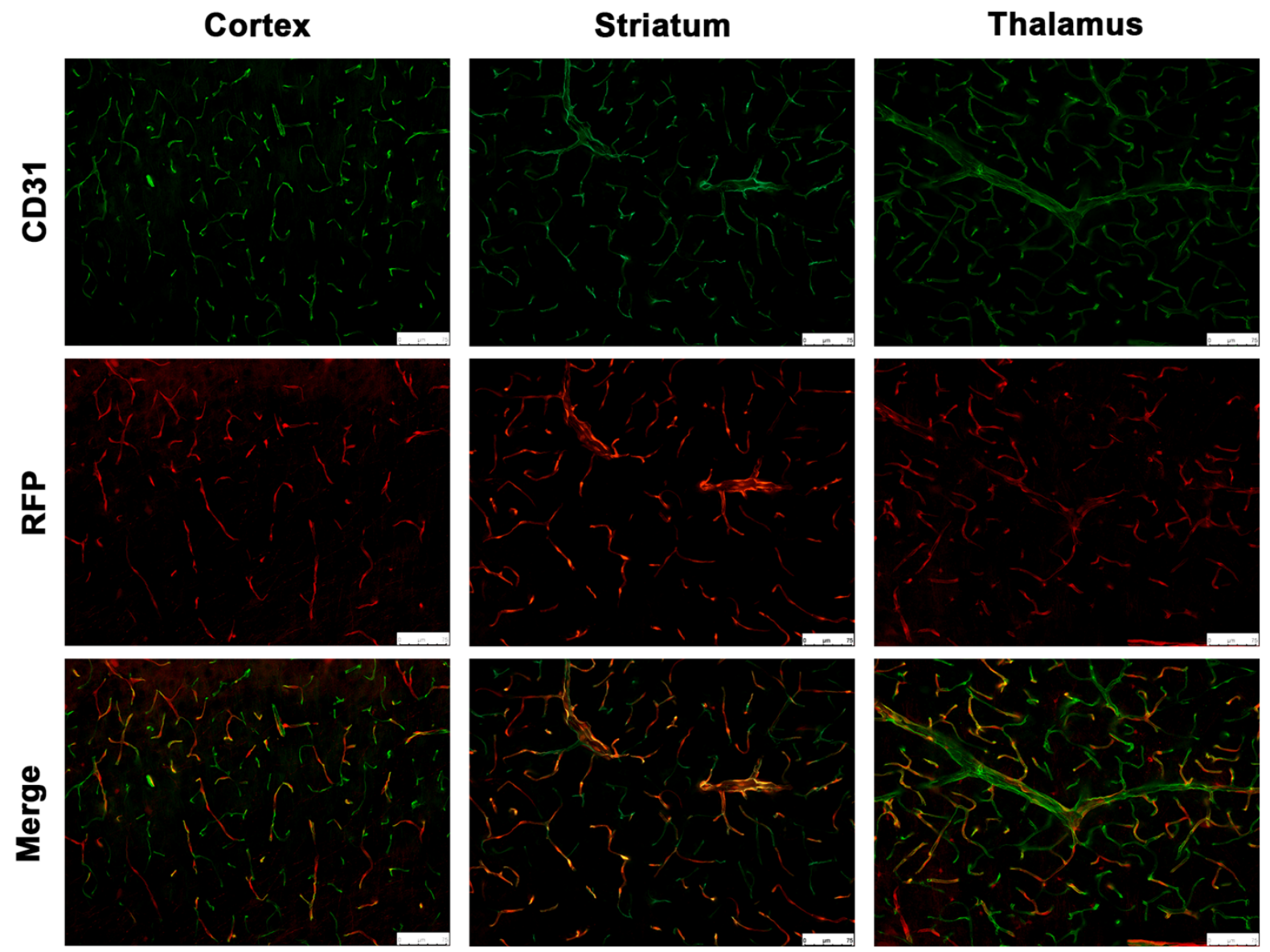


Supplementary Figure 4

a

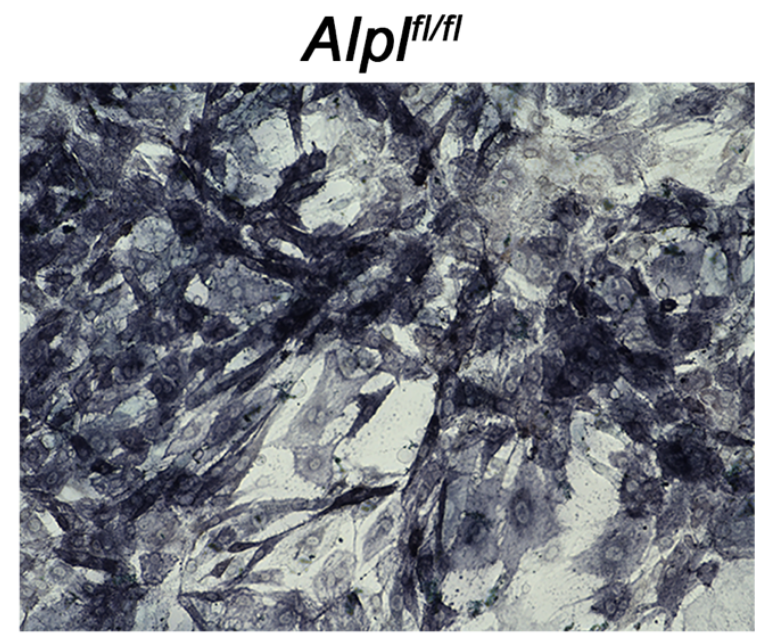

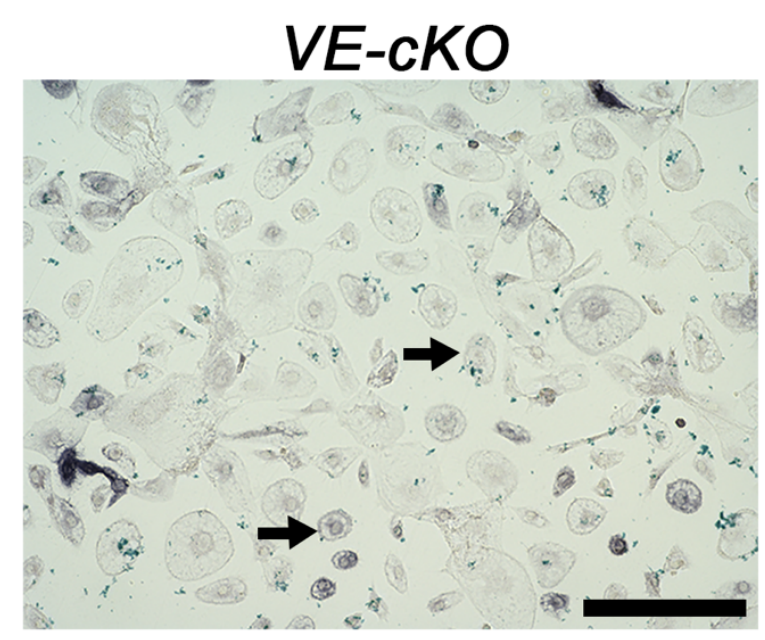

b
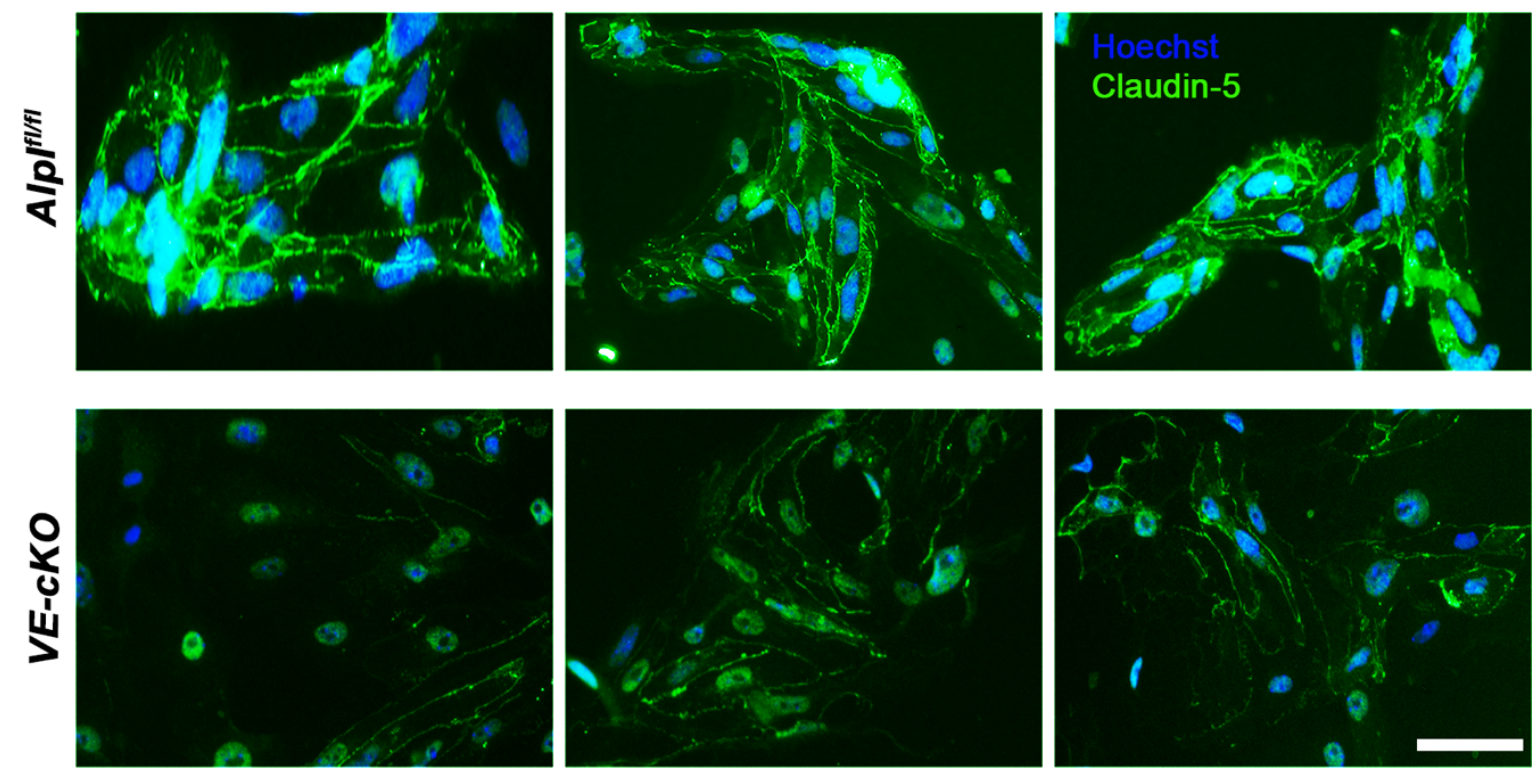


\section{Supplementary Figure Legends}

Supplementary Figure 1: Expression of brain microvascular TNAP activity is region specific in mice. (A) Representative images of brain microvascular TNAP activity expression in the cortex, striatum, and hippocampus (CA3 shown) of WT mice $(n=5)$. (B) Quantification of brain microvascular TNAP activity expression revealed that TNAP activity is most significantly expressed in the striatum ( $p<0.0001$, Tukey's multiple comparisons test, one-way ANOVA) and cortex ( $p=0.0002$, Tukey's multiple comparisons test, one-way ANOVA) relative to the hippocampus. Interestingly, TNAP activity in the striatum was significantly increased $(p=0.03)$ when compared to the cortex. ${ }^{*}$ indicates $p<0.05,{ }^{* * *} p<0.001$, and ${ }^{* * *} p<0.0001$, and is considered significant. All data are presented as mean \pm SEM. Images taken at $20 X$ magnification and scale bar $=200 \mu \mathrm{m}$.

Supplementary Figure 2: Loss of TNAP activity on brain microvessels. (A) Representative images showed loss of TNAP activity on CD31 positive (black arrow) brain microvessels 7 days post-sepsis. (B) Similarly, TNAP activity on CD31 positive brain microvessels decreased in the ipsilateral striatal penumbra (stroke hemisphere) compared to the contralateral striatum (nonstroke hemisphere) 7 days post-stroke. Images taken at 20X magnification and scale bar $=250$ $\mu \mathrm{m}$.

Supplementary Figure 3: VE-Cadherin Cre is specific for microvessels. Red fluorescent protein (RFP) signal colocalizes with CD31 vascular endothelial marker. Cortex, striatum, and thalamus are shown. Images taken at 20X magnification and scale bar $=75 \mu \mathrm{m}$

Supplementary Figure 4: pBMEC morphology and junctional protein expression. (A) Representative images showed increased cell detachment and endothelial retraction (black arrows) in VE-cKO pBMECs compared to AlpI/f/fl pBMECs. (B) Claudin-5 junctional protein expression is decreased in VE-cKO pBMECs compared to Alpl ${ }^{\text {fl/fl }}$ pBMECs. Images taken at 20X magnification and scale bar $=200 \mu \mathrm{m}(\mathrm{A})$; Images taken at 40X magnification and scale bar $=$ $100 \mu \mathrm{m}(\mathrm{B})$. 


\section{References}

1. Kishnani, P.S., et al., Five-year efficacy and safety of asfotase alfa therapy for adults and adolescents with hypophosphatasia. Bone, 2019. 121: p. 149-162.

2. Banks, W.A., From blood-brain barrier to blood-brain interface: new opportunities for CNS drug delivery. Nat Rev Drug Discov, 2016. 15(4): p. 275-92.

3. Nwafor, D.C., et al., Targeting the Blood-Brain Barrier to Prevent Sepsis-Associated Cognitive Impairment. J Cent Nerv Syst Dis, 2019. 11: p. 1179573519840652.

4. Daneman, R. and A. Prat, The blood-brain barrier. Cold Spring Harb Perspect Biol, 2015. 7(1): p. a020412.

5. Varatharaj, A. and I. Galea, The blood-brain barrier in systemic inflammation. Brain Behav Immun, 2017. 60: p. 1-12.

6. Yanagida, K., et al., Size-selective opening of the blood-brain barrier by targeting endothelial sphingosine 1-phosphate receptor 1. Proc Natl Acad Sci U S A, 2017. 114(17): p. 4531-4536.

7. Obermeier, B., R. Daneman, and R.M. Ransohoff, Development, maintenance and disruption of the blood-brain barrier. Nat Med, 2013. 19(12): p. 1584-96.

8. Heemskerk, S., et al., Alkaline phosphatase treatment improves renal function in severe sepsis or septic shock patients. Crit Care Med, 2009. 37(2): p. 417-23, e1.

9. Rader, B.A., Alkaline Phosphatase, an Unconventional Immune Protein. Front Immunol, 2017. 8: p. 897.

10. Buchet, R., J.L. Millan, and D. Magne, Multisystemic functions of alkaline phosphatases. Methods Mol Biol, 2013. 1053: p. 27-51.

11. Brun-Heath, I., et al., Differential expression of the bone and the liver tissue non-specific alkaline phosphatase isoforms in brain tissues. Cell Tissue Res, 2011. 343(3): p. 521-36.

12. Farkas-Bargeton, E. and M.L. Arsenio-Nunes, [Maturation of enzymatic equipment in the vessel walls of the nervous system. Histochemical study]. Acta Neuropathol, 1970. 15(3): p. 251-71.

13. Williams, S.K., et al., Isolation and characterization of brain endothelial cells: morphology and enzyme activity. J Neurochem, 1980. 35(2): p. 374-81.

14. Picher, M., et al., Ecto 5'-nucleotidase and nonspecific alkaline phosphatase. Two AMPhydrolyzing ectoenzymes with distinct roles in human airways. J Biol Chem, 2003. 278(15): p. 13468-79.

15. Peters, E., et al., Alkaline phosphatase protects against renal inflammation through dephosphorylation of lipopolysaccharide and adenosine triphosphate. Br J Pharmacol, 2015. 172(20): p. 4932-45.

16. Bentala, H., et al., Removal of phosphate from lipid $A$ as a strategy to detoxify lipopolysaccharide. Shock, 2002. 18(6): p. 561-6.

17. Bates, J.M., et al., Intestinal alkaline phosphatase detoxifies lipopolysaccharide and prevents inflammation in zebrafish in response to the gut microbiota. Cell Host Microbe, 2007. 2(6): p. 371-82.

18. Peters, E., et al., Alkaline phosphatase: a possible treatment for sepsis-associated acute kidney injury in critically ill patients. Am J Kidney Dis, 2014. 63(6): p. 1038-48.

19. Eltzschig, H.K., M.V. Sitkovsky, and S.C. Robson, Purinergic signaling during inflammation. N Engl J Med, 2012. 367(24): p. 2322-33.

20. Bours, M.J., et al., Adenosine 5'-triphosphate and adenosine as endogenous signaling molecules in immunity and inflammation. Pharmacol Ther, 2006. 112(2): p. 358-404.

21. Michie, H.R., et al., Detection of circulating tumor necrosis factor after endotoxin administration. N Engl J Med, 1988. 318(23): p. 1481-6. 
22. Nwafor, D.C., et al., Loss of tissue-nonspecific alkaline phosphatase (TNAP) enzyme activity in cerebral microvessels is coupled to persistent neuroinflammation and behavioral deficits in late sepsis. Brain Behav Immun, 2020. 84: p. 115-131.

23. Fonta, C., et al., Rediscovering TNAP in the Brain: A Major Role in Regulating the Function and Development of the Cerebral Cortex. Subcell Biochem, 2015. 76: p. 85106.

24. Fonta, C., et al., Postnatal development of alkaline phosphatase activity correlates with the maturation of neurotransmission in the cerebral cortex. J Comp Neurol, 2005. 486(2): p. 179-96.

25. Teriete, P., A.B. Pinkerton, and N.D. Cosford, Inhibitors of tissue-nonspecific alkaline phosphatase (TNAP): from hits to leads. Methods Mol Biol, 2013. 1053: p. 85-101.

26. Pinkerton, A.B., et al., Discovery of 5-((5-chloro-2methoxyphenyl)sulfonamido)nicotinamide (SBI-425), a potent and orally bioavailable tissue-nonspecific alkaline phosphatase (TNAP) inhibitor. Bioorg Med Chem Lett, 2018. 28(1): p. 31-34.

27. Foster, B.L., et al., Conditional Alpl Ablation Phenocopies Dental Defects of Hypophosphatasia. J Dent Res, 2017. 96(1): p. 81-91.

28. Weksler, B.B., et al., Blood-brain barrier-specific properties of a human adult brain endothelial cell line. FASEB J, 2005. 19(13): p. 1872-4.

29. Alva, J.A., et al., VE-Cadherin-Cre-recombinase transgenic mouse: a tool for lineage analysis and gene deletion in endothelial cells. Dev Dyn, 2006. 235(3): p. 759-67.

30. Benjamin, E.J., et al., Heart Disease and Stroke Statistics-2018 Update: A Report From the American Heart Association. Circulation, 2018. 137(12): p. e67-e492.

31. Madisen, L., et al., A robust and high-throughput Cre reporting and characterization system for the whole mouse brain. Nat Neurosci, 2010. 13(1): p. 133-40.

32. Brichacek, A.L., et al., Systemic inhibition of tissue-nonspecific alkaline phosphatase alters the brain-immune axis in experimental sepsis. Sci Rep, 2019. 9(1): p. 18788.

33. Doll, D.N., et al., Lipopolysaccharide exacerbates infarct size and results in worsened post-stroke behavioral outcomes. Behav Brain Funct, 2015. 11(1): p. 32.

34. Ma, H.W., et al., In-Cell Western Assays to Evaluate Hantaan Virus Replication as a Novel Approach to Screen Antiviral Molecules and Detect Neutralizing Antibody Titers. Front Cell Infect Microbiol, 2017. 7: p. 269.

35. Kilkenny, C., et al., Animal research: reporting in vivo experiments: the ARRIVE guidelines. Br J Pharmacol, 2010. 160(7): p. 1577-9.

36. Engelhardt, B. and S. Liebner, Novel insights into the development and maintenance of the blood-brain barrier. Cell Tissue Res, 2014. 355(3): p. 687-99.

37. Deracinois, B., et al., Tissue Non-specific Alkaline Phosphatase (TNAP) in Vessels of the Brain. Subcell Biochem, 2015. 76: p. 125-51.

38. Debray, J., et al., Inhibitors of tissue-nonspecific alkaline phosphatase: design, synthesis, kinetics, biomineralization and cellular tests. Bioorg Med Chem, 2013. 21(24): p. 7981-7.

39. Hall, A., Rho GTPases and the actin cytoskeleton. Science, 1998. 279(5350): p. 509-14.

40. Xing, L. and D.G. Remick, Mechanisms of dimethyl sulfoxide augmentation of IL-1 beta production. J Immunol, 2005. 174(10): p. 6195-202.

41. Koch, J.C., et al., ROCK inhibition in models of neurodegeneration and its potential for clinical translation. Pharmacol Ther, 2018. 189: p. 1-21.

42. M.J. Weiss, K.R., P.S. Henthorn, B. Lamb, T. Kadesch, H. Harris, Structure of the human liver/bone/kidney alkaline phosphatase gene. JOURNAL OF BIOLOGICAL CHEMISTRY, 1988. 263: p. 12002-10. 
43. Yang, J., F. Ruan, and Z. Zheng, Ripasudil Attenuates Lipopolysaccharide (LPS)Mediated Apoptosis and Inflammation in Pulmonary Microvascular Endothelial Cells via ROCK2/eNOS Signaling. Med Sci Monit, 2018. 24: p. 3212-3219.

44. Grothaus, J.S., et al., Rho kinase inhibition maintains intestinal and vascular barrier function by upregulation of occludin in experimental necrotizing enterocolitis. Am J Physiol Gastrointest Liver Physiol, 2018. 315(4): p. G514-G528.

45. Feng, S., et al., RhoA/ROCK-2 Pathway Inhibition and Tight Junction Protein Upregulation by Catalpol Suppresses Lipopolysaccaride-Induced Disruption of BloodBrain Barrier Permeability. Molecules, 2018. 23(9).

46. Barone, F.C., et al., Tumor necrosis factor-alpha. A mediator of focal ischemic brain injury. Stroke, 1997. 28(6): p. 1233-44.

47. Romero, C.R., et al., The role of interferon-gamma in the pathogenesis of acute intraabdominal sepsis. J Leukoc Biol, 2010. 88(4): p. 725-35.

48. Yilmaz, G., et al., Role of T lymphocytes and interferon-gamma in ischemic stroke. Circulation, 2006. 113(17): p. 2105-12.

49. Mehra, A., et al., Nonionotropic Action of Endothelial NMDA Receptors on Blood-Brain Barrier Permeability via Rho/ROCK-Mediated Phosphorylation of Myosin. J Neurosci, 2020. 40(8): p. 1778-1787.

50. Schubert-Unkmeir, A., et al., Neisseria meningitidis induces brain microvascular endothelial cell detachment from the matrix and cleavage of occludin: a role for MMP-8. PLoS Pathog, 2010. 6(4): p. e1000874.

51. Greene, C., N. Hanley, and M. Campbell, Claudin-5: gatekeeper of neurological function. Fluids Barriers CNS, 2019. 16(1): p. 3.

52. Esue, O., et al., A direct interaction between actin and vimentin filaments mediated by the tail domain of vimentin. J Biol Chem, 2006. 281(41): p. 30393-9.

53. Svitkina, T.M., A.B. Verkhovsky, and G.G. Borisy, Plectin sidearms mediate interaction of intermediate filaments with microtubules and other components of the cytoskeleton. J Cell Biol, 1996. 135(4): p. 991-1007.

54. Hollenbeck, P.J., et al., Intermediate filament collapse is an ATP-dependent and actindependent process. J Cell Sci, 1989. 92 ( Pt 4): p. 621-31.

55. Dupin, I., Y. Sakamoto, and S. Etienne-Manneville, Cytoplasmic intermediate filaments mediate actin-driven positioning of the nucleus. J Cell Sci, 2011. 124(Pt 6): p. 865-72.

56. Jiu, Y., et al., Bidirectional Interplay between Vimentin Intermediate Filaments and Contractile Actin Stress Fibers. Cell Rep, 2015. 11(10): p. 1511-8.

57. Eriksson, J.E., et al., Introducing intermediate filaments: from discovery to disease. J Clin Invest, 2009. 119(7): p. 1763-71.

58. Yang, L., et al., Raf-1/CK2 and RhoA/ROCK signaling promote TNF-alpha-mediated endothelial apoptosis via regulating vimentin cytoskeleton. Toxicology, 2017. 389: p. 7484.

59. Trindade, P., et al., Short and long TNF-alpha exposure recapitulates canonical astrogliosis events in human-induced pluripotent stem cells-derived astrocytes. Glia, 2020. 68(7): p. 1396-1409.

60. Shi, J., et al., Distinct roles for ROCK1 and ROCK2 in the regulation of cell detachment. Cell Death Dis, 2013. 4: p. e483.

61. Shibuya, M., et al., Effects of fasudil in acute ischemic stroke: results of a prospective placebo-controlled double-blind trial. J Neurol Sci, 2005. 238(1-2): p. 31-9.

62. Fukuta, T., et al., Combination therapy with liposomal neuroprotectants and tissue plasminogen activator for treatment of ischemic stroke. FASEB J, 2017. 31(5): p. 18791890.

63. Liu, K., et al., Role of rho kinase in microvascular damage following cerebral ischemia reperfusion in rats. Int J Mol Sci, 2011. 12(2): p. 1222-31. 
64. Zhu, J., et al., Exploring the beneficial role of ROCK inhibitors in sepsis-induced cerebral and cognitive injury in rats. Fundam Clin Pharmacol, 2021. 


\section{Chapter 4}

\section{A Brief Report: The in vivo effects of a conditional loss of brain endothelial cell tissue-nonspecific alkaline phosphatase (TNAP) on blood-brain barrier permeability in the early stages of injury}

Divine C. Nwafor ${ }^{a}$ Allison L. Brichacek ${ }^{b}$, Luke Cox $^{a}$, José Luis Millán ${ }^{c}$, and Candice M.
Brown ${ }^{a, b^{*}}$

${ }^{a}$ Department of Neuroscience, West Virginia University Health Science Center, Morgantown, WV 26506, USA

${ }^{b}$ Department of Microbiology, Immunology, and Cell Biology, School of Medicine, West Virginia University Health Science Center, Morgantown, WV 26506, USA

'Sanford-Burnham Prebys Medical Discovery Institute, La Jolla, CA 92037, USA

Nwafor DC, Brichacek AL, Cox L, Millan JL, Brown CM. The in vivo effects of a conditional loss of brain endothelial cell tissue-nonspecific alkaline phosphatase (TNAP) on blood-brain barrier permeability in early sepsis. (In preparation)

Note: Parts of this chapter include text and figures taken from the publication highlighted above 


\subsection{Abstract}

Blood-brain barrier (BBB) dysfunction is a key feature seen across many neuroinflammatory conditions. Recent studies suggest that BBB dysfunction precedes long-term cognitive impairment seen across many neuroinflammatory diseases. Yet, no therapies exist to effectively mitigate BBB dysfunction. Preliminary evidence suggests that tissue-nonspecific alkaline phosphatase (TNAP), an ectoenzyme localized to cerebral microvessels, may play an important role in maintaining barrier integrity. In a recent study, we demonstrated that loss of TNAP in primary microvascular endothelial cell (pBMEC) cultures worsened barrier integrity. However, it remained unclear whether these findings translate in vivo. In the present study, we sought to investigate the role of BMEC TNAP in maintaining BBB integrity in health and during sepsis. We hypothesized that a conditional loss of brain endothelial TNAP in cerebral microvessels would exacerbate BBB permeability to fluorescent molecules in naïve VE-cKO mice. Our results revealed that naïve VE-cKO demonstrated a size selective increased permeability to small molecules such as Rhodamine 123 (380 Da) but not to Texas red (625 Da). More importantly, when sepsis or stroke is initiated in VE-cKO mice, BBB permeability is significantly increased. Taken together, we demonstrate a novel role for BMEC TNAP in maintaining barrier integrity in health and during systemic inflammation.

Keywords: Tissue-nonspecific alkaline phosphatase; Alpl; Blood-brain barrier, BBB Permeability; Brain microvascular endothelial cell; Sepsis 


\subsection{Introduction}

The blood-brain barrier (BBB) is a selective vascular interface composed of brain microvascular endothelial cells (BMECs), pericytes, basement membrane, and astrocyte endfeet processes [1, 2]. BBB dysfunction is a key neuropathological feature seen in many neuroinflammatory diseases [3]. Yet, therapies that mitigate BBB dysfunction remain to be elucidated despite recent studies suggesting that BBB dysfunction may be responsible for longterm cognitive impairment [4-6]. To elucidate the role of the BBB in disease progression, it is imperative that researchers examine protein/enzymes localized to cells that comprise the BBB such as the BMEC.

Tissue-nonspecific alkaline phosphatase (TNAP) is one of many proteins localized to BMECs, and is highly abundant in human and rodent cerebral microvessels [7]. Recent studies from our group have demonstrated that following sepsis, TNAP activity was decreased in cerebral microvessels and this decrease in TNAP activity was coupled to increased BBB permeability to immunoglobulin G [8] [9]. Furthermore, we showed that pharmacological inhibition of TNAP activity in hCMEC/D3 cells or conditional loss of brain endothelial TNAP (VEcKO) in primary cultures diminished paracellular barrier integrity using in vitro and ex vivo barrier assays [10]. However, it is not clear whether the findings of diminished barrier seen in VE-cKO primary brain microvascular endothelial cell (pBMECs) culture translate in vivo (i.e., in the context of the BBB) given that endothelial cell solo-cultures lose their BBB properties and characteristics [11].

The objective of this study was to examine the role of brain endothelial TNAP in health and sepsis. We hypothesized that a conditional loss of brain endothelial TNAP in cerebral microvessels would exacerbate BBB permeability to fluorescent molecules in VE-cKO naïve and septic mice. First, we assessed permeability of the BBB in naïve VE-cKO mice using fluorescent dyes of different sizes. Thereafter, we initiated sepsis in our mice via the cecal ligation and puncture (CLP) model of sepsis, and then assessed BBB permeability to fluorescent dyes $24 \mathrm{~h}$ 
post-sepsis. Furthermore, we examined whether VE-cKO stroke mice exhibit worsened BBB dysfunction and neuroinflammation compared to $A / p f^{f / f l}$ stroke mice. Taken together, our results demonstrate that loss of brain endothelial TNAP in vivo resulted in a size selective whole brain permeability in naïve VE-cKO mice that is exacerbated in septic and stroke. Moreover, our finding provides preclinical evidence for the use of TNAP therapeutics to mitigate BBB dysfunction seen in sepsis and stroke.

\subsection{Methods}

\subsubsection{Animals}

Experiments were conducted in accordance with the National Institutes of Health Guide for the Care and Use of Laboratory Animals and were approved by the Institutional Animal Care and Use Committee at West Virginia University. Creation of $A / p f^{f / f f}$ mice is described in [12] and creation of VE-Cadherin Cre mice is described in [13]. Floxed $A / p l\left(A / p f^{f / f f}\right)$ mice on a C57BL/6J genetic were crossed with B6.FVB-Tg (Cdh5-cre)7Mlia/J (VE-Cadherin Cre, Bar Harbor, ME, Catalog \# 006137) to ultimately produce mice with a conditional deletion of $A / p /$ in the endothelium (VE-cKO) and littermate control mice $\left(A / p /^{f / f f}\right)$. Genotyping was performed as described previously [10]. Briefly, DNA was extracted from ear snips using the Purelink Genomic DNA Mini Kit (Invitrogen, Carlsbad, CA, USA), and PCR products were amplified by using a Veriti 96-well Thermal Cycler (Applied Biosystems, ThermoFisher Scientific, Waltham, MA) under the following conditions: $94^{\circ} \mathrm{C}$ for $1 \mathrm{~min},\left[\left(94^{\circ} \mathrm{C}\right.\right.$ for $30 \mathrm{sec}, 60^{\circ} \mathrm{C}$ for $30 \mathrm{sec}, 72^{\circ} \mathrm{C}$ for $45 \mathrm{sec}) \times$ cycles], then $72^{\circ} \mathrm{C}$ for $1 \mathrm{~min}$. VE-Cadherin Cre specificity was determined by the presence of a 700 bp product using the following primers; ACRE_F: 5'-

GAACCTGATGGACATGTTCAGGGA -3', and ACRE_R: 5'-

CAGAGTCATCCTTAGCGCCGTAAA -3' [13]. Confirmation of floxed Alp/ sites was determined by the presence of a 263 bp product using the following primer set; Alplflox_F: 5'GTTGCGATGTGTGAAGATGTCCTCG -3', and Alplflox_R: 5’- 
CTTGGGCTTGCTGTCGCCAGTAAC -3'. All mice were group housed in environmentally controlled conditions with a reverse light cycle $\left(12: 12 \mathrm{~h}\right.$ light/dark cycle at $\left.21 \pm 1^{\circ} \mathrm{C}\right)$ and provided food and water ad libitum. Male and female wild-type mice were bred in West Virginia University Health Sciences Center vivarium facilities and both sexes were used in all experiments. Mice 3-4 months old were used for naïve and stroke experiments while 11-12 months old were used for sepsis experiments. Aged mice were used in the septic experiments due to prior studies demonstrating that middle aged and elderly individuals are at a higher risk for sepsis mortality and morbidity [14-16].

\subsubsection{Cecal Ligation and Puncture (CLP)}

The cecal ligation and puncture (CLP) model of polymicrobial sepsis was employed as previously described $[9,17]$. Briefly, $A / p f^{f / f l}$ and VE-cKO mice were anesthetized by the inhalation of $1-2 \%$ isoflurane and abdominal access was obtained via a midline incision. The cecum was ligated below the ileocecal valve, punctured twice with a $22 \mathrm{G}$ needle through and

through, and placed back into the abdominal cavity. The abdominal muscle and skin layer were closed with 6-0 and 5-0 sutures (Ethilon, Cornelia, GA) respectively. Sham-operated animals had their cecum isolated and then returned to the peritoneal cavity without being ligated or punctured. One $\mathrm{mL}$ of sterile $0.9 \%$ saline was administered subcutaneously (s.c.) for fluid resuscitation in all experimental groups. Mice used for all experiments were euthanized at $24 \mathrm{~h}$ post-sepsis surgery for permeability studies.

\subsubsection{Permanent Middle Cerebral Artery Occlusion (pMCAO)}

pMCAO surgery was performed under isoflurane anesthesia as previously described [18]. Briefly, Alp $f^{f / f f l}$ and VE-cKO mice were subjected to pMCAO using silicon coated sutures (Cat. \#702334, Doccol Corporation, MA) followed by reperfusion. Body temperatures were controlled at $37 \pm 0.5^{\circ} \mathrm{C}$ during occlusion. Occlusion was verified in each animal by a Laser 
Speckle Imager (Moor Instruments, England). Bupivacaine ( $2 \mathrm{mg} / \mathrm{kg}$, s.c.) was administered to relieve pain after surgery. Mice were euthanized $6 \mathrm{~h}$ following pMCAO surgery.

\subsubsection{BBB Permeability Assay, Perfusion, and Tissue Collection}

Rhodamine 123 (Invitrogen, Carlsbad, CA) and Texas red (Invitrogen) fluorescent dyes were used to measure permeability as described previously [19]. Briefly, both dyes were coinjected at a concentration of $2 \mathrm{mM}$ intravenously via the retro-orbital vein. Following retroorbital injection, the dyes were allowed to circulate for 15 mins prior to euthanasia. Mice were then deeply anesthetized with isoflurane after 15 mins of dye circulation and transcardially perfused (Masterflex 7524-10, Cole-Parmer, Vernon Hills, IL) with 0.9\% saline to remove blood. Thereafter, brains were removed from the skull with the olfactory bulbs and cerebellum carefully dissected out and discarded. The right cerebrum and left cerebrum were quickly separated. The left cerebrum was immediately frozen in $-80^{\circ} \mathrm{C}$ isopentane for $1 \mathrm{~min}$ and then stored at $-20^{\circ} \mathrm{C}$ until further processing. The right cerebrum was weighed (grams) and placed in microcentrifuge tubes (VWR, Bridgeport, NJ) containing $300 \mu \mathrm{L}$ of phosphate buffered saline (PBS) for homogenization. Following homogenization, samples were then centrifuged at $15000 \mathrm{~g}, 20$ mins, at $4^{\circ} \mathrm{C}$. Supernatants were transferred into a new microcentrifuge tube (see Figure 1A). A similar experimental paradigm as shown in Figure 1A was carried out for sham and septic experiments, however, cascade blue-3 kDa dextran dye (Invitrogen) was utilized in addition to the Rhodamine 123 and Texas red fluorescent. Furthermore, dyes were allowed to circulate for $1 \mathrm{~h}$ instead of 15 mins.

\subsubsection{BBB permeability quantification}

\subsubsection{Spatial permeability}

Spatial/regional permeability was assessed in the left cerebrum of naive animals as previously described [19]. Frozen brains were sectioned at $20 \mu$ on a Leica CM30505 cryostat 
(Leica Biosystems, Buffalo Grove, IL). Regions such as the cortex, striatum, hippocampus, and thalamus were identified using the Allen Institute Brain Atlas (http://mouse.brain-map.org). 3 sections per brain region from each animal were used for all image analyses. Fluorescence intensity was measured on the Olympus MVS10 stereomicroscope (Olympus, Japan) using a 2X objective and an optical zoom 0.63 to $6.3 X$. Rhodamine 123 and Texas red excitation/emission filters (470/525 $\mathrm{nm}$ and $560 / 645 \mathrm{~nm}$ respectively) were used to acquire fluorescence intensity.

\subsubsection{Whole cerebrum (brain) permeability}

Whole brain permeability was assessed in the right cerebrum of naïve and septic animals as previously described [19]. Briefly, $50 \mu \mathrm{L}$ triplicates of tissue supernatant from the right cerebrum was pipetted into a 96-well plate. The 96-well plate was inserted into a plate reader (BioTek, Winooski, VT) and fluorescence was measured using respective excitation/emission filters for Rhodamine (488/525 nm), Texas red (461/605 nm), and cascade blue-3 kDa dextran $(360 / 450 \mathrm{~nm})$ dyes. Relative fluorescence intensity was normalized to the weight of the right cerebrum.

\subsubsection{Stroke brain collection, immunohistochemistry, and image analysis}

Mice were deeply anesthetized with isoflurane and transcardially perfused as described previously [9]. Briefly, blood was removed with $0.9 \%$ saline followed by perfusion and fixation with $4 \%$ chilled paraformaldehyde (PFA, Fisher Scientific, Pittsburgh, PA). Perfused brains were post-fixed in $4 \%$ PFA overnight at $4^{\circ} \mathrm{C}$. On the following day, brains were rinsed in $0.01 \mathrm{M}$ PBS and incubated sequentially in $15 \%$ and $30 \%$ sucrose in PBS for $24 \mathrm{~h}$ each. Brains were sectioned at $35 \mu \mathrm{m}$ on a sliding microtome (HM 450, ThermoFisher Scientific). Brain sections were washed three times, permeabilized, and blocked for 30 min on a shaker. Brain sections were then incubated overnight with primary antibodies, followed by a $2 \mathrm{~h}$ incubation with the 
appropriate secondary antibody at room temperature. The following primary antibodies were used with working dilutions and antibody identification indicated in parentheses: GFAP (Agilent (1:10,000), AB_2811722, Santa Clara, CA) and Fibrinogen (Abcam (1:500), (AB_732367), Cambridge, MA). Sections were viewed on a Leica DM6B microscope (Leica Camera, Allendale, NJ) and images were captured using Leica LASX software (Leica Microsystems, Buffalo Grove, IL). Cortex was identified by referring to the Allen Institute Brain Atlas (http://mouse.brain-map.org). Three random images were collected per animal per brain region of interest. All collected images were converted to an 8-bit image and quantified in FIJI/Image $\mathrm{J}$ version 2.0 software as percent area.

\subsubsection{Statistical Analysis}

Experiments were executed to enhance rigor and avoid experimenter bias according to ARRIVE guidelines [20]. Animals whose brain did not perfuse well were removed from the study as described in [19]. All statistical analyses were performed in GraphPad Prism 9.0 (GraphPad Software, La Jolla, CA). Results are expressed as mean \pm SEM and $p$-values $\leq 0.05$ were considered significant. Data was analyzed using a two-tailed unpaired Student's t-test, repeated two-way analysis of variance (ANOVA), or two-way analysis of variance followed by Tukey's multiple comparisons post hoc test. Datasets that did not display a Gaussian distribution were subjected to a comparable non-parametric analysis (Mann-Whitney test). All $p$-values and $n$ values are indicated in the figure legends. Outliers were removed using the GraphPad prism Grubbs outlier test.

\subsection{Results}

4.4.1. Naïve VE-cKO mice whole brain demonstrated a size selective permeability to Rhodamine 123 (380 Da) but not to Texas red (625 Da). 
First, we assessed whether the loss of brain endothelial TNAP would increase BBB permeability to fluorescent dyes of different sizes. Our results showed that VE-cKO mice revealed no difference in Rhodamine 123 or Texas red spatial permeability in the cortex. striatum, hippocampus, and thalamus when compared to Alpfl/fl mice (Fig. 1B-G; Supplementary Fig.1). Next, we assessed whether VE-cKO mice would demonstrate increased whole brain permeability when compared to $A / p f^{f / f f l}$ mice. Our results showed that VE-cKO mice revealed a significantly increased permeability to Rhodamine 123 but $(p=0.03)$ not to Texas red $(p=0.24)$

(Fig. 2). Taken together, our results suggest that loss of brain endothelial TNAP results in a size selective loss of whole brain BBB integrity.

\subsubsection{Loss of brain endothelial TNAP worsened BBB permeability to fluorescent dyes post- sepsis}

Next, we assessed whether the increase in whole brain permeability to small molecules seen in naïve VE-cKO mice would be exacerbated in early sepsis (24 h). Paradoxically, Rhodamine 123 permeability was significantly decreased (main effect: $p=0.003$ ) in the brains of VE-cKO and Alp $f^{f / f f l}$ septic mice (Fig. 3A). Post hoc analyses revealed that the decrease in Rhodamine 123 permeability post-sepsis was only significant between $A / f^{f / / f l}$ sham and Alp fl/fl septic mice $(p=0.0028)$ but not between VE-cKO sham and VE-cKO septic mice $(p=0.32)$. Sepsis significantly increased permeability to Texas red (main effect: $p<0.0005$ ) and cascade blue-3 kDa dextran (main effect: $p<0.0021$ ) dyes for both VE-cKO and Alp/f/ffl mice. Post-hoc analyses revealed that VE-cKO septic mice when compared to VE-cKO sham mice demonstrated a significantly increased permeability to Texas red $(p=0.0022)$ and cascade blue-3 kDa dextran $(p=0.03)$. However, no differences in permeability to Texas red $(p=0.33)$ and cascade blue-3 kDa dextran $(p=0.24)$ were seen between male Alp $f^{f / f f l}$ sham and Alp/f/ffl septic mice. More importantly, comparisons between VE-cKO sham and $A / p f^{f l / f l}$ sham mice revealed no difference in Texas red $(p=0.99)$ or cascade blue $(p=0.99)$ permeability (Fig. 3 B, 
C). These results suggest a plausible role for TNAP in maintaining BBB permeability during systemic inflammation.

\subsubsection{VE-cKO stroke mice displayed increased fibrinogen extravasation and astrocyte} proliferation

Thereafter, we examined whether VE-cKO mice subjected to $6 \mathrm{~h}$ pMCAO would exhibit a worsened BBB integrity compared to $A / p I^{f / f t}$ stroke mice. Our results showed that VE-ckO stroke mice demonstrated a significant increase $(p=0.050)$ in brain parenchymal fibrinogen deposition compared to $A / p I^{f / f t}$ stroke mice. Furthermore, the increase in fibrinogen brain deposition ( $p=$ 0.046) was coupled to significant astrogliosis in VE-cKO (Fig. 4).

\subsection{Discussion}

TNAP activity is abundantly expressed in cerebral microvessels, however, its function on cerebral microvessels has been vastly unexplored until lately. We recently demonstrated that inhibition of TNAP activity in an immortalized human endothelial cell line (hCMEC/D3) or a conditional loss of TNAP in pBMECs resulted in a loss of barrier integrity [10]. Though these results have advanced our knowledge on the function of TNAP in BMECs, it remained unclear whether the observed in vitro findings of diminished barrier integrity would persist in vivo.

In the present study, we sought to examine whether conditional loss of BMEC TNAP in vivo would exacerbate BBB permeability in naïve and septic mice. We showed here that whole brain BBB permeability following a loss of BMEC TNAP was increased to smaller molecules $(\sim 380 \mathrm{Da})$ but not to molecules greater or equal to $625 \mathrm{Da}$. The findings from this study resemble the deletion of junctional protein claudin- 5 in mice which led to a size selective permeability to small molecules $<800 \mathrm{Da}$ [21]. Interestingly, both TNAP and claudin-5 global knockouts die immediately after birth which further highlights the critical role played by both proteins in normal physiology $[21,22]$. Furthermore, a recent study showed that size selective permeability to small molecules in the early phase of ischemic brain injury is attributed to actin 
cytoskeletal re-organization [23]. We have previously shown that inhibition of BMEC TNAP led to actin cytoskeletal re-organization via the ROCK pathway [10]. Thus, it is likely that loss of BMEC TNAP in vivo and the resultant size selective permeability to small molecules may be mediated through actin cytoskeletal re-organization.

Spatial permeability was also assessed in naïve mice; however, no differences were observed indicating that whole brain rather than regional permeability needs to be taken into account when studying BMEC TNAP function in vivo. It is unclear whether BBB permeability to very small molecules like Rhodamine 123 (380 Da) holds any biological significance in healthy mice. Future studies will need to address whether naïve VE-cKO mice exhibit cognitive decline in young and aged mice. Additionally, whole brain selective permeability to only small molecules in naïve mice suggest that other cell types of the BBB may compensate for BMEC TNAP loss through molecular cross-talk via signaling molecules. For instance, co-cultures of BMECs and mixed glial cells in vitro has been shown to drastically increase TNAP protein, activity, and mRNA expression in BMECs compared to solo-cultures [24-26]. More importantly, astrocyte end-feet processes and pericytes have been shown to express TNAP activity [27-29]. Hence, it becomes plausible that the loss of cerebral microvascular TNAP activity seen in sepsis may have resulted from a combined loss of TNAP activity in pericytes or astrocytes rather than BMECs alone. Future studies are warranted to examine how TNAP expression in other cell types of the BBB work together with BMEC TNAP to maintain precise cerebral homeostasis.

Since naïve mice demonstrated a size selective permeability to smaller molecules, we examined the implications of this size selective permeability in the context of systemic inflammation (i.e., sepsis). Not surprising, there was a significant main effect of treatment (sepsis) that led to an increased permeability of fluorescent dyes in VE-cKO and Alp $f^{f / f f l}$ septic mice compared to controls. These findings are corroborated by other septic studies which have shown increased BBB dysfunction post-sepsis [30, 31]. Interestingly, post-hoc analyses revealed that VE-cKO septic mouse exhibited a significantly increased permeability to Texas red 
(625 Da) and cascade blue-3 kDa dextran when compared to VE-cKO sham mice. However, no significant differences in permeability were seen between $A / p f^{f / f f l}$ sham and $A / p f^{f / f f l}$ septic mice when compared. Next, we examined the loss of BMEC in a model of stroke. As expected, VEcKO stroke mice exhibited a worsened barrier integrity denoted by increased fibrinogen extravasation into the brain parenchyma. Therefore, our results suggest that the size selective permeability seen in naïve VE-cKO mice is worsened when sepsis or stroke is superimposed on an already leaky BBB.

Paradoxically, Rhodamine 123 was decreased in the brains of septic mice compared to sham mice. We speculate that the decrease in Rhodamine 123 in the brains of septic mice likely resulted from the $1 \mathrm{~h}$ dye circulatory period prior to euthanasia. Furthermore, inflammatory molecules have been shown to increase P-glycoprotein (P-gp) efflux transporter expression in the kidney, pulmonary, and intestinal tissue [32, 33]. Given that Rhodamine 123 is a P-gp substrate [34], it is likely that this dye would be rapidly removed from the brain by the P-gp efflux transporter if allowed to circulate for longer periods (> 15 mins) post-sepsis. Interestingly, posthoc analyses revealed that the significant decrease in Rhodamine 123 is only present between $A l p f^{f / f f l}$ sham and $A l p f^{f / f l}$ septic mice but not VE-cKO sham and VE-cKO septic mouse. These findings suggest that P-gp efflux transporter function may be dysfunctional in VE-cKO mice following sepsis.

In summary, our results demonstrate a novel role for BMEC TNAP in maintaining barrier integrity in vivo that corroborates our recent in vitro findings [10]. Furthermore, we have demonstrated a plausible role for the P-gp efflux transporter post-sepsis, which may yield therapeutic consideration when selecting drugs to treat sepsis associated brain dysfunction. Further investigations that examine the contribution of other BBB TNAP expressing cell types such as astrocytes and pericytes in maintaining BBB integrity are needed to fully elucidate the role of TNAP in cerebral microvessels. 


\subsection{Author contributions}

D.C.N, A.L.B, and C.M.B designed the studies. D.C.N and L.C. sectioned all tissues. D.C.N and L.C performed image analyses. D.C.N and A.L.B performed sepsis surgeries and permeability experiments. A.L.B. generated and genotyped $A / f^{f / f f l}$ and VE-cKO animals needed for experiments. D.C.N. and performed statistical analyses of data and generation of graphs. D.C.N. and C.M.B. wrote the manuscript. JL.M. provided $A / p f^{f / f f}$ mice. All authors read and revised the final manuscript.

Acknowledgement: The authors gratefully thank the West Virginia University Office of Laboratory Animal Resources and the West Virginia University Experimental Stroke Core for their assistance with stroke surgeries. Funding for this work was supported by the NIH T32 AG052375 (D.C.N, A.L.B), K01 NS081014 (C.M.B), West Virginia Clinical and Translational Science Institute (U54 GM104942), and the West Virginia University Stroke CoBRE (P20 GM109098). 
Figures

Figure 1

a.

1. Retro-orbital injection of mouse and dye

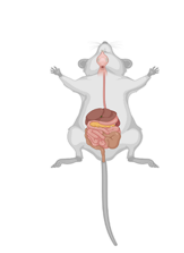$$
\text { . }
$$
2. Mouse is perfused
and brain is collected

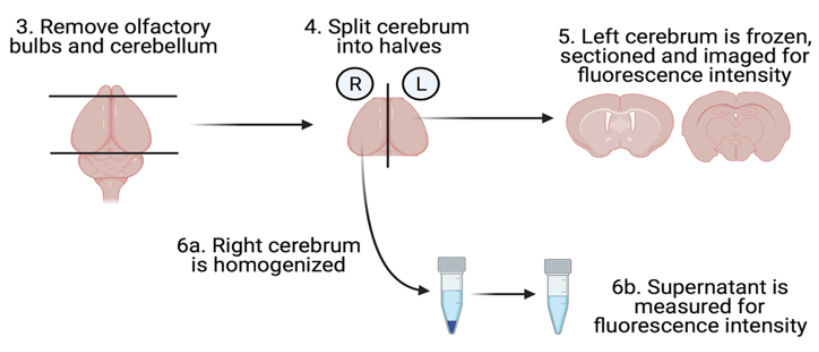

b.

Rhodamine 123 (380 Da)
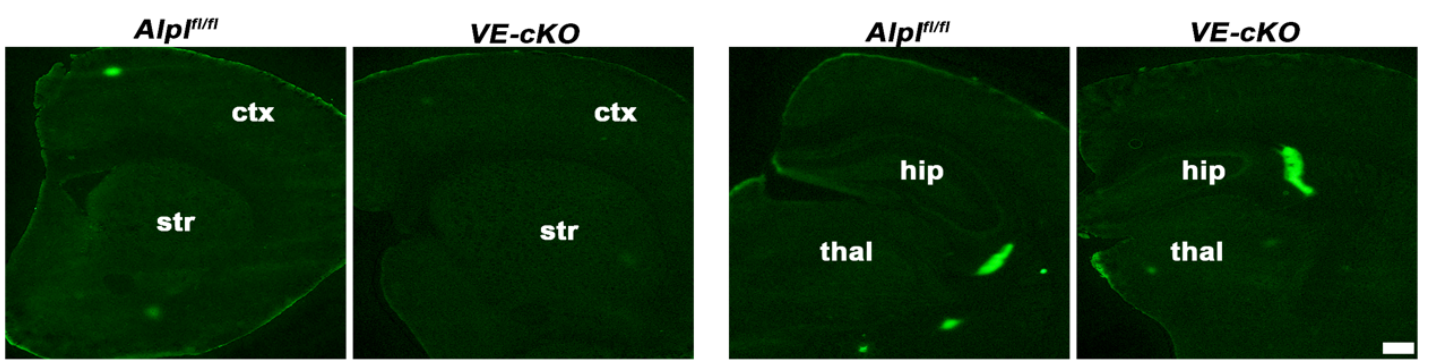

c.
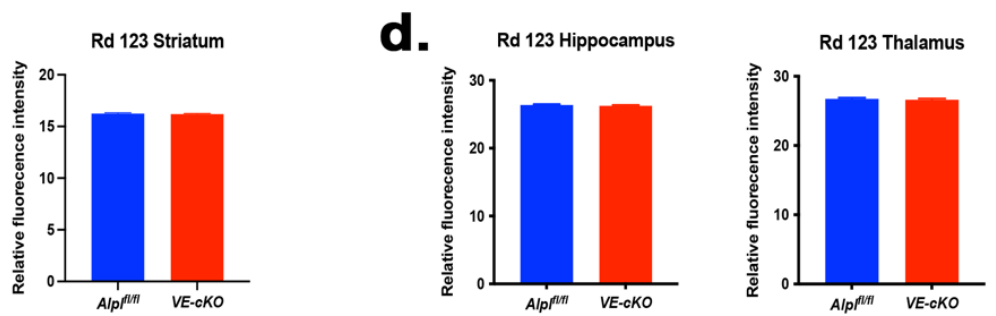

e.

Texas Red (625 Da)
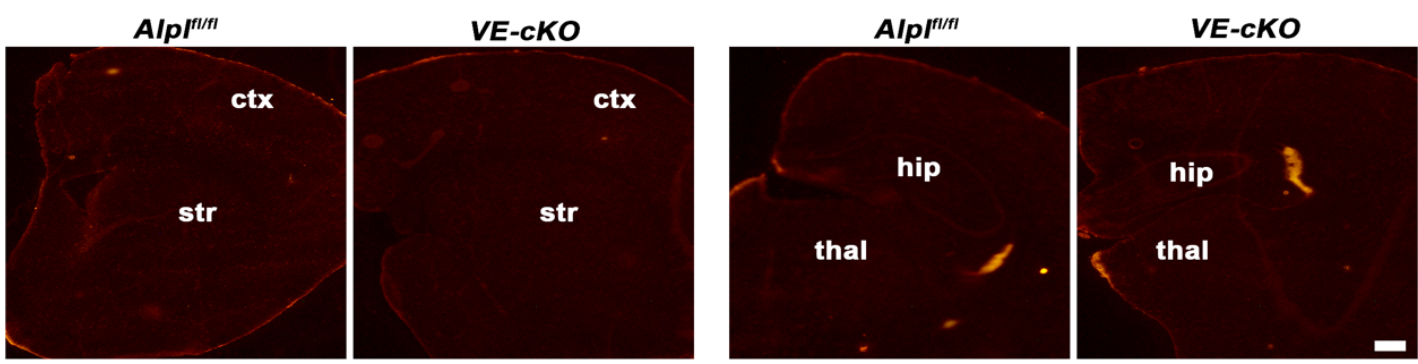

f.

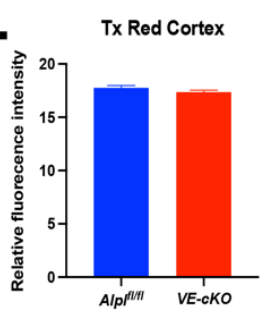

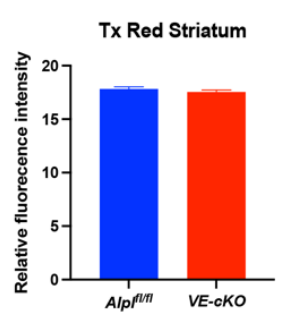

g.

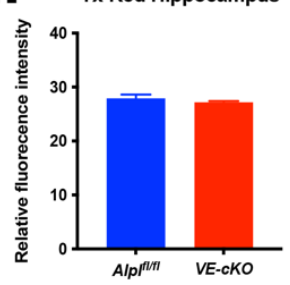

Tx Red Thalamus

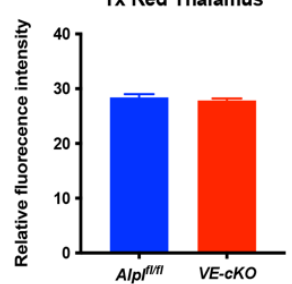


Figure 2

a.

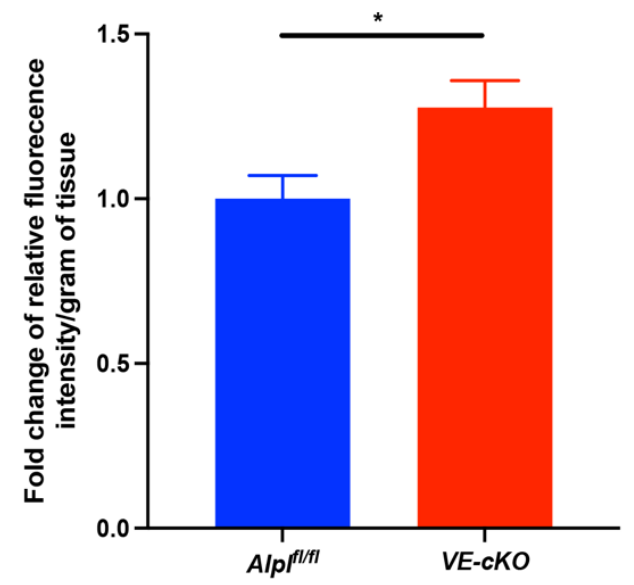

b.

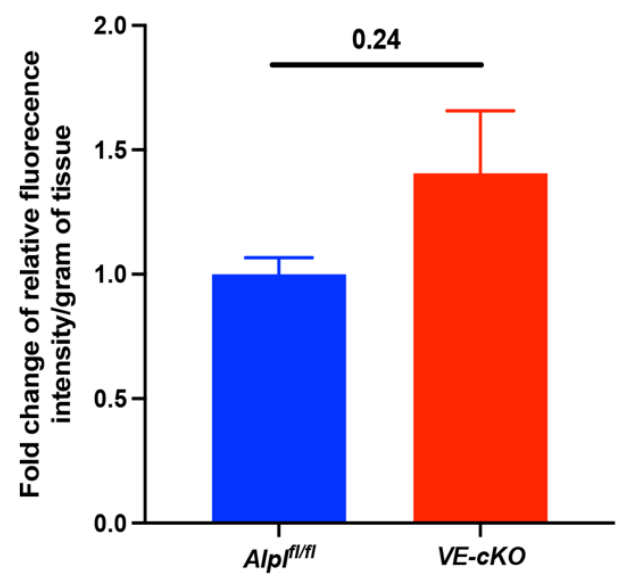


Figure 3

a.

Rhodamine 123

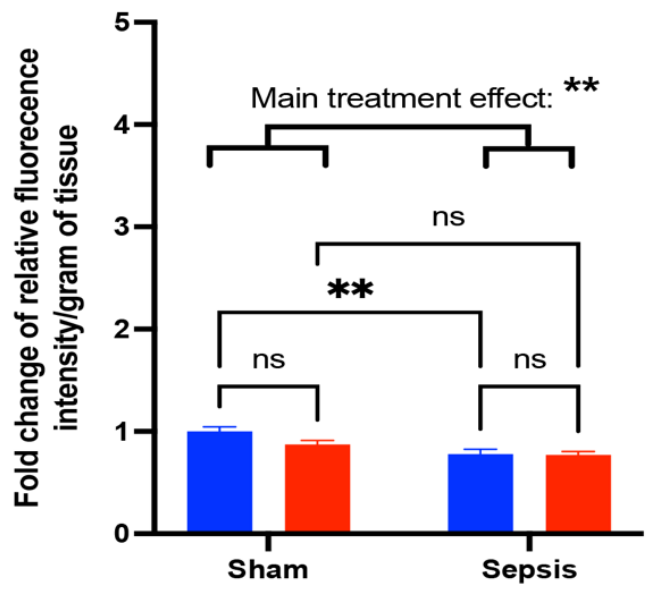

b.

Texas Red

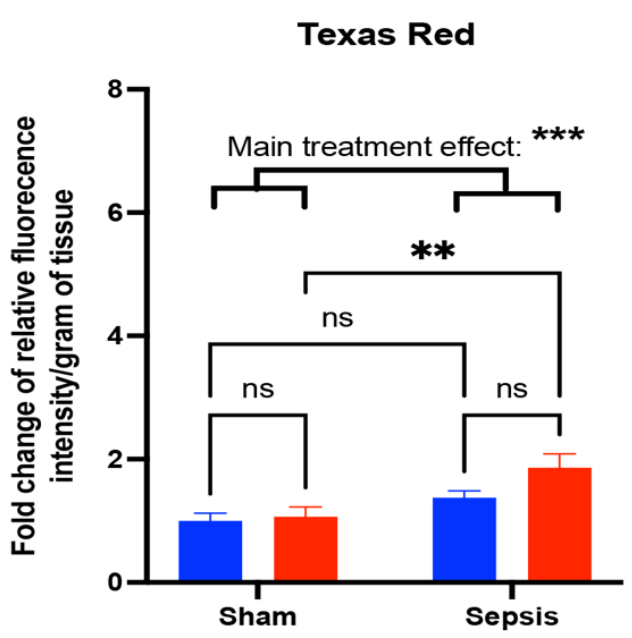

Alpf $f^{f / f l}$

VE-cKO

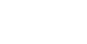

c.

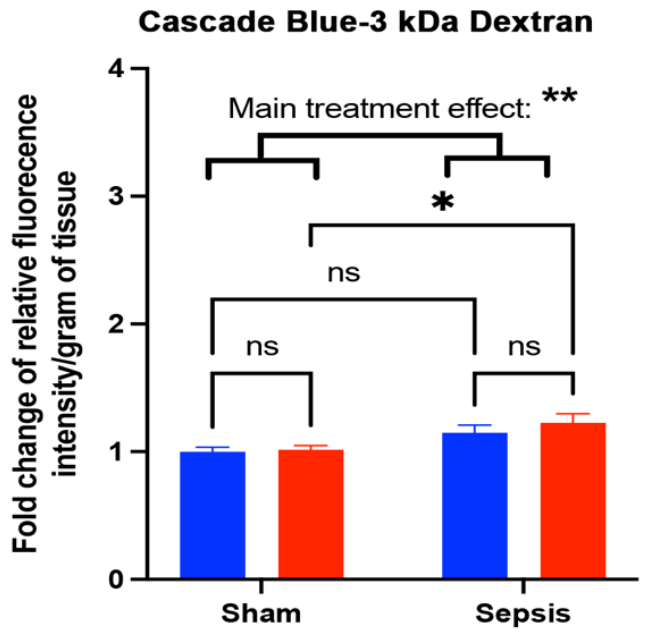

Alpfflfl

VE-CKO 
Figure 4
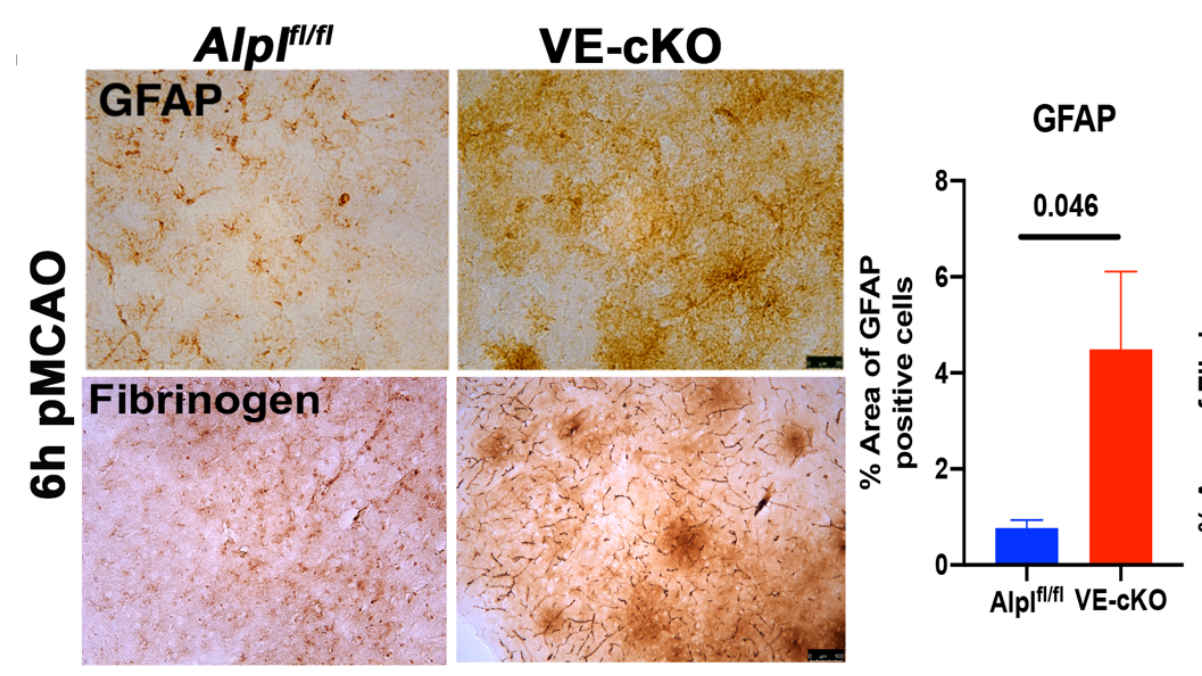

Fibrinogen

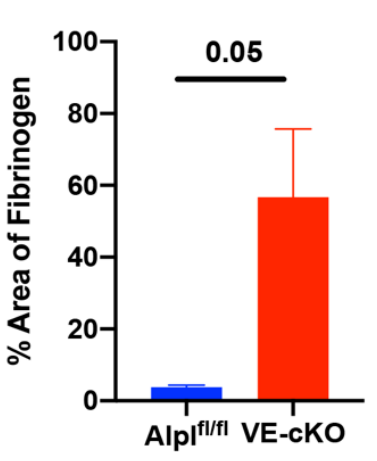




\section{Figure Legends}

Figure 1: Naïve VE-cKO mice displayed no differences in spatial BBB permeability. (A) Experimental design for the naïve BBB permeability study. (B-G) Cortex (ctx), striatum (str), hippocampus (hip), and thalamus (thal) revealed no differences in spatial permeability to Rhodamine $123(p>0.05)$ or Texas red $(p>0.05)$ fluorescent dyes between naive VE-cKO and Alp $f^{f / f l}$ mice. A two-tailed unpaired Student's t-test was used for all analyses. All data presented as mean \pm SEM. Images taken at $1.5 \mathrm{X}$ magnification and scale bar $=1 \mathrm{~mm}$. $n=6 /$ mice group.

Figure 2: Naïve VE-cKO mice demonstrate a whole brain size selective permeability to Rhodamine 123. (A) Rhodamine 123 (380 Da) whole brain permeability was significantly increased ( $p=0.03$; two-tailed unpaired Student's t-test) in VE-cKO mice compared to Alp f/ffl mice. (B) Texas red (625 Da) permeability showed no significant differences $(p>0.05$; MannWhitney test) between naive VE-cKO and $A / p f^{f / f f l}$ mice. ${ }^{*}$ indicates $p<0.05$ and is considered significant. All data presented as mean \pm SEM. $n=6 /$ mice group.

Figure 3: Sepsis exacerbates VE-cKO size selective permeability. (A) Rhodamine 123 permeability was significantly decreased (main treatment effect: $p=0.003$; two-way ANOVA) in the brains of VE-cKO and Alp/fl/fl septic mice. Tukey's multiple comparisons test revealed that the decrease in Rhodamine 123 permeability post-sepsis was only significant $(p=0.0028)$ between $A / p f^{f / f f l}$ sham and $A / p f^{f / f l}$ septic mice. (B-C) Sepsis significantly increased permeability to Texas red (main treatment effect: $p<0.0005$; two-way ANOVA) and cascade blue-3 kDa dextran (main treatment effect: $p<0.0021$; two-way ANOVA) dyes for both VE-cKO and Alpfl/fl mice. Tukey's multiple comparisons test revealed that VE-cKO septic mice when compared to VE-cKO sham mice demonstrated a significantly increased permeability to Texas red $(p=$ $0.0022)$ and cascade blue-3 kDa dextran $(p=0.03)$. Alp $f^{f l / f l}$ sham and Alpff/fli septic mice Tukey's multiple comparisons revealed no significant differences in permeability to Texas red $(p=0.33)$ and cascade blue $(p=0.24)$ dyes. ${ }^{*}$ indicates $p<0.05,{ }^{* *} p<0.01{ }^{* * *} p<0.001$, and is considered significant. All data presented as mean \pm SEM. $n=13-20 /$ mice group. $n s=$ not significant.

Figure 4: VE-cKO stroke mice displayed BBB dysfunction and astrogliosis. Brains of VEcKO stroke mice exhibited a significant increase in astrogliosis $(p=0.046)$ and fibrinogen $(p=$ 0.05 ) deposition compared to $A / p f^{f / f l}$ mice. Scale bar $=75 \mu \mathrm{m}$. A two-tailed unpaired Student's ttest was used for all analyses All data presented as mean \pm SEM. $n=2 /$ mice group. 


\section{Supplementary Figure}
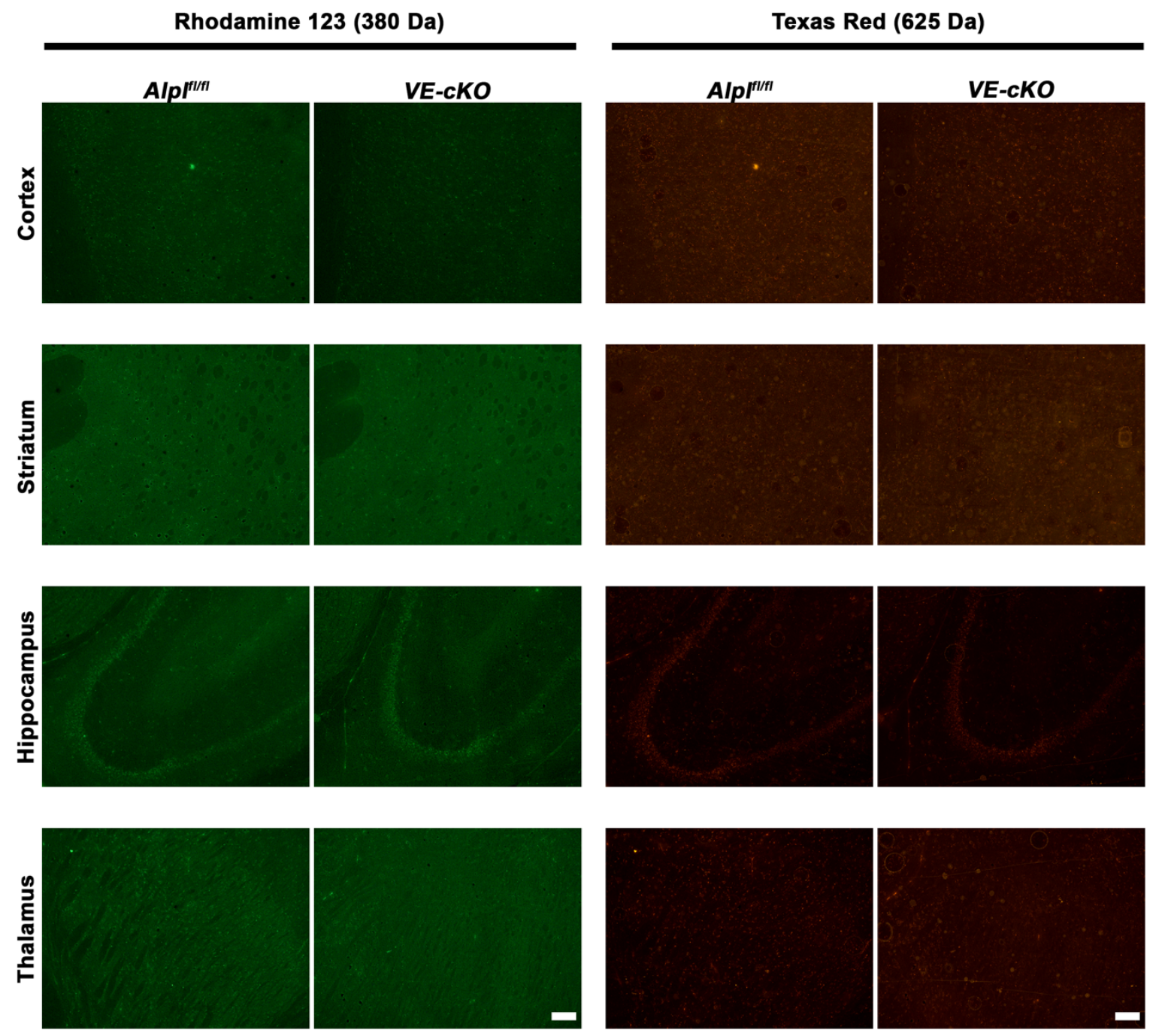


\section{Supplementary Figure Legends}

Supplemental Figure 1 VE-cKO mice displayed no differences in spatial BBB

permeability. 10X magnified images further demonstrated that Cortex, striatum, hippocampus (CA3 shown), and thalamus revealed no differences in spatial permeability to Rhodamine 123 or Texas red fluorescent dyes between naive VE-cKO and $A / p f^{f / t / t}$ mice. Scale bar $=100 \mu \mathrm{m}$. 


\section{Reference}

1. Nwafor, D.C., et al., Targeting the Blood-Brain Barrier to Prevent Sepsis-Associated Cognitive Impairment. J Cent Nerv Syst Dis, 2019. 11: p. 1179573519840652.

2. Banks, W.A., From blood-brain barrier to blood-brain interface: new opportunities for CNS drug delivery. Nat Rev Drug Discov, 2016. 15(4): p. 275-92.

3. Varatharaj, A. and I. Galea, The blood-brain barrier in systemic inflammation. Brain Behav Immun, 2017. 60: p. 1-12.

4. Montagne, A., et al., Blood-brain barrier breakdown in the aging human hippocampus. Neuron, 2015. 85(2): p. 296-302.

5. Nation, D.A., et al., Blood-brain barrier breakdown is an early biomarker of human cognitive dysfunction. Nat Med, 2019. 25(2): p. 270-276.

6. $\quad$ Cramer, S.P., et al., Abnormal blood-brain barrier permeability in normal appearing white matter in multiple sclerosis investigated by MRI. Neuroimage Clin, 2014. 4: p. 182-9.

7. Deracinois, B., et al., Tissue Non-specific Alkaline Phosphatase (TNAP) in Vessels of the Brain. Subcell Biochem, 2015. 76: p. 125-51.

8. Michie, H.R., et al., Detection of circulating tumor necrosis factor after endotoxin administration. N Engl J Med, 1988. 318(23): p. 1481-6.

9. Nwafor, D.C., et al., Loss of tissue-nonspecific alkaline phosphatase (TNAP) enzyme activity in cerebral microvessels is coupled to persistent neuroinflammation and behavioral deficits in late sepsis. Brain Behav Immun, 2020. 84: p. 115-131.

10. Nwafor, D.C., et al., Brain endothelial cell tissue-nonspecific alkaline phosphatase (TNAP) activity promotes maintenance of barrier integrity via the ROCK pathway. 2021: bioRxiv.

11. Helms, H.C., et al., In vitro models of the blood-brain barrier: An overview of commonly used brain endothelial cell culture models and guidelines for their use. J Cereb Blood Flow Metab, 2016. 36(5): p. 862-90.

12. Foster, B.L., et al., Conditional Alpl Ablation Phenocopies Dental Defects of Hypophosphatasia. J Dent Res, 2017. 96(1): p. 81-91.

13. Alva, J.A., et al., VE-Cadherin-Cre-recombinase transgenic mouse: a tool for lineage analysis and gene deletion in endothelial cells. Dev Dyn, 2006. 235(3): p. 759-67.

14. Iwashyna, T.J., et al., Long-term cognitive impairment and functional disability among survivors of severe sepsis. JAMA, 2010. 304(16): p. 1787-94.

15. Calsavara, A.J.C., et al., Factors Associated With Short and Long Term Cognitive Changes in Patients With Sepsis. Sci Rep, 2018. 8(1): p. 4509.

16. Mankowski, R.T., et al., Older Sepsis Survivors Suffer Persistent Disability Burden and Poor Long-Term Survival. J Am Geriatr Soc, 2020. 68(9): p. 1962-1969.

17. Brichacek, A.L., et al., Systemic inhibition of tissue-nonspecific alkaline phosphatase alters the brain-immune axis in experimental sepsis. Sci Rep, 2019. 9(1): p. 18788.

18. Doll, D.N., et al., Lipopolysaccharide exacerbates infarct size and results in worsened post-stroke behavioral outcomes. Behav Brain Funct, 2015. 11(1): p. 32.

19. Devraj, K., et al., An In Vivo Blood-brain Barrier Permeability Assay in Mice Using Fluorescently Labeled Tracers. J Vis Exp, 2018(132).

20. Kilkenny, C., et al., Improving bioscience research reporting: the ARRIVE guidelines for reporting animal research. PLoS Biol, 2010. 8(6): p. e1000412.

21. Nitta, T., et al., Size-selective loosening of the blood-brain barrier in claudin-5-deficient mice. J Cell Biol, 2003. 161(3): p. 653-60.

22. Buchet, R., J.L. Millan, and D. Magne, Multisystemic functions of alkaline phosphatases. Methods Mol Biol, 2013. 1053: p. 27-51. 
23. Shi, Y., et al., Rapid endothelial cytoskeletal reorganization enables early blood-brain barrier disruption and long-term ischaemic reperfusion brain injury. Nat Commun, 2016. 7: p. 10523.

24. Deracinois, B., et al., TNAP and EHD1 are over-expressed in bovine brain capillary endothelial cells after the re-induction of blood-brain barrier properties. PLoS One, 2012. 7(10): p. e48428.

25. Meyer, J., J. Rauh, and H.J. Galla, The susceptibility of cerebral endothelial cells to astroglial induction of blood-brain barrier enzymes depends on their proliferative state. J Neurochem, 1991. 57(6): p. 1971-7.

26. Rauh, J., et al., Development of an in vitro cell culture system to mimic the blood-brain barrier. Prog Brain Res, 1992. 91: p. 117-21.

27. Mayahara, H., et al., The new lead citrate method for the ultracytochemical demonstration of activity of non-specific alkaline phosphatase (orthophosphoric monoester phosphohydrolase). Histochemie, 1967. 11(1): p. 88-96.

28. Mori, S. and M. Nagano, Electron-microscopic cytochemistry of alkaline-phosphatase activity in endothelium, pericytes and oligodendrocytes in the rat brain. Histochemistry, 1985. 82(3): p. 225-31.

29. Ovtscharoff, W., [Ultracytochemical localisation of the alkaline phosphatase in the cerebral cortex of newborn rats (author's transl)]. Histochemie, 1973. 37(1): p. 93-5.

30. Kikuchi, D.S., et al., Poldip2 mediates blood-brain barrier disruption in a model of sepsisassociated encephalopathy. J Neuroinflammation, 2019. 16(1): p. 241.

31. Towner, R.A., et al., Assessing long-term neuroinflammatory responses to encephalopathy using MRI approaches in a rat endotoxemia model. Geroscience, 2018. 40(1): p. 49-60.

32. Ibrahim, Y.F., et al., Tocilizumab attenuates acute lung and kidney injuries and improves survival in a rat model of sepsis via down-regulation of NF-kappaB/JNK: a possible role of P-glycoprotein. Inflammopharmacology, 2020. 28(1): p. 215-230.

33. Moriguchi, J., et al., Effects of lipopolysaccharide on intestinal P-glycoprotein expression and activity. Eur J Pharmacol, 2007. 565(1-3): p. 220-4.

34. Jouan, E., et al., Evaluation of P-Glycoprotein Inhibitory Potential Using a Rhodamine 123 Accumulation Assay. Pharmaceutics, 2016. 8(2). 


\section{Chapter 5}

\section{Discussion}

Nwafor, DC., Brichacek, AL., Ahsan, A., \& Brown, CM. TNAP in Central Nervous System

Health and Disease: A Focus on Brain Microvascular Endothelial Cells. International Journal of Molecular Sciences. 2021.

Nwafor DC, Brown CM. A novel role for tissue-nonspecific alkaline phosphatase at the bloodbrain barrier during sepsis. Neural Regen Res. 2021;16(1):99-100. doi:10.4103/16735374.286958

Nwafor, D.C, Brichacek, A.L, Chakraborty, S., Gambill, C., Benkovic, S., \& Brown, C.M. (2020, March 31). Neuroendocrine and Neuroimmune Mechanisms Regulating the BloodBrain Barrier. Oxford Research Encyclopedia of Neuroscience. doi:

10.1093/acrefore/9780190264086.013.307

Note: Parts of this chapter include text and figures taken from the publications highlighted above 
Brain microvascular endothelial cells (BMECs) are a key cellular component of the blood-brain barrier (BBB) that provide a stringent, yet, dynamic interface between systemic circulation and the brain parenchyma [1, 2]. BMECs are characterized by a non-fenestrated continuous endothelium linked together by tight junction [3] proteins that confer high resistance to paracellular transport between adjacent endothelial cells [4-6]. BMEC dysfunction is seen across several neuroinflammatory diseases including sepsis, stroke, multiple sclerosis (MS), and Alzheimer's disease (AD). Elegant studies have shown that BBB dysfunction precedes cognitive decline or disease activity in AD, MS, and aging [7-12]. TNAP is one of many proteins localized to BMECs, and is highly abundant in human and rodent cerebral microvessels [13]. Despite the high expression of TNAP on cerebral microvessels, the role of TNAP in the brain microvascular endothelium remained unclear until recently [13-17]. The overarching objective of

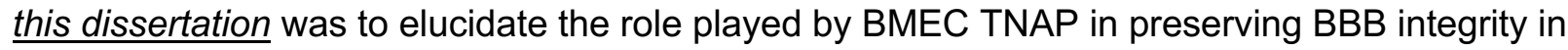
normal physiology and in sepsis. Three sets of studies were designed to address the knowledge gaps associated with understanding the function of TNAP in cerebral microvessels - specifically BMECs. We proposed a central hypothesis that BMEC TNAP is critical for maintenance of BBB integrity in normal physiology and in sepsis through molecular mechanisms that preserve BMEC structure and function.

In this body of work, we showed that BMEC TNAP activity plays a critical role in maintaining paracellular barrier integrity in health and in disease (sepsis and stroke). Prior to this work, the role for BMEC TNAP in cerebral microvessel was vastly unexplored. Previous studies from decades ago had suggested that the appearance of TNAP activity in cerebral microvessels during development correlated with a functional BBB [13]. However, these studies did not examine how TNAP contributed to proper BBB integrity and function. A critical question in the field that remained unanswered was whether the loss of TNAP activity seen in disease coupled BBB dysfunction. Indeed, we showed in Chapters 2 and 3 that TNAP activity was coupled to BBB dysfunction in sepsis and stroke. Furthermore, we provided substantial 
evidence in Chapter 2 that pro-inflammatory cytokines may be responsible for the decrease in BMEC TNAP activity seen in sepsis and stroke. More importantly, our studies in Chapter 3 significantly contributed to the field by mechanistically delineating a molecular target of TNAP ROCK. Lastly, we investigated the role of BMEC TNAP in vivo in sepsis and stroke. We showed that loss of BMEC TNAP in sepsis and stroke worsened BBB dysfunction. This is the first in vivo study to provide preliminary evidence for TNAP as a therapeutic target to treat cerebral injury.

One of the many challenges in studying organ specific TNAP function arises from the fact that life expectancy of mice with a global genetic deletion of the Alpl gene $\left(\mathrm{Alp} \mathrm{F}^{-H^{-}}\right)$averages 3-5 weeks due to hypophosphatasia (HPP) and neurological deficits such as seizures [18, 19]. However, development of the Alpl floxed mice by Dr. Jose Luis Millan and VE-Cadherin Cre mice by Alva et el. have aided our understanding of TNAP in BMECs [20-23]. The data described in this dissertation demonstrates the utility of the VE-CKO mice as a mouse model that could be used to investigate BMEC TNAP function in future studies.

\section{A novel anti-inflammatory role for TNAP in cerebral microvessels}

Historically, TNAP activity has been used as a histological marker for the presence or absence of cerebral vessels in disease without much attention given to why the loss of TNAP activity was occurring, and how this loss might affect disease progression [13]. This perspective is exemplified in an earlier study utilizing TNAP activity to examine vascular density [24]. Briefly, Brown et al. investigated brain arteriolar and capillary density in 12 patients with leukoaraisosis (LA; age-related white matter degenerative disease) and 9 age-matched control patients using TNAP activity. The authors conclude that vascular density is significantly decreased in LA when compared to control patients [24]. However, it was not evident from the study why TNAP activity was decreased in patients with LA compared to controls. It was also not clear if the loss of TNAP activity in LA was due to the loss of cerebral microvessels since the study did not utilize 
appropriate markers for the vasculature such as CD31, CD34, or collagen type IV [25]. Owing to these findings, several important questions were raised for future investigations:

1) Is the loss of TNAP activity on cerebral microvessels specific to a unitary disease state?

2) Does the loss of TNAP activity equate to the loss of cerebral microvessels?

3) Does the loss of TNAP in cerebral microvessels couple the loss of barrier integrity?

4) What are the relevant long-term neuroinflammatory and behavioral implications of brain microvascular TNAP loss?

The results derived in chapter 2 of this dissertation sought to answer these critical questions. A significant number of studies have demonstrated a role for peripheral endothelial TNAP activity in disease [26-29], however, there is a paucity of data on the function of cerebral microvascular TNAP activity in disease. To elucidate whether TNAP activity was altered in disease, we utilized the cecal ligation and puncture (CLP) model of sepsis to examine how TNAP activity changed in early sepsis and whether this changed was sustained long-term (7 days). Our results showed that loss of TNAP activity is present as early as $24 \mathrm{~h}$ post-sepsis [30] and this loss was sustained up to 7 days post-sepsis [15]. However, it was unclear whether the resultant loss of TNAP activity was due to cerebral microvessel loss as stated in the Brown et al. LA study [24]. Therefore, we co-labelled for cerebral microvessel using CD31 and TNAP activity. Our results showed that indeed the loss of TNAP activity was not due to a loss of brain microvessels. This finding refutes the utility of brain microvascular TNAP activity as a measure of vascular density since this activity changes with disease. More importantly, we showed that the loss of TNAP activity on cerebral microvessels coupled a loss of junctional protein claudin-5, microglial activation, astrocyte proliferation, and the infiltration of an endogenous permeability marker, immunoglobulin G [31], into the brain [15]. To further demonstrate a role for brain microvascular TNAP in disease, we intraperitoneally injected septic mice with SBI-425 (an in 
vivo TNAP inhibitor) or vehicle (DMSO) and examined junctional protein claudin-5 expression. Our results showed that claudin-5 expression was decreased in SBI-425 treated septic mice compared to vehicle treated septic mice.

Sepsis survivors are burdened with long-term cognitive impairments [32], hence, we examined whether the loss of TNAP in cerebral microvessels coupled behavioral deficits at 7 days post-sepsis. We showed that our septic mice displayed an impaired sensorimotor phenotype but had no alterations in memory. We speculate that the impairment in sensorimotor dysfunction likely results from the loss of TNAP in the cortex, striatum, and spinal cord, whereas, no differences in memory were observed because hippocampal TNAP activity at 7day post-sepsis revealed no differences between sham and septic animals [15]. Nevertheless, it remains unclear whether hippocampal TNAP activity may be altered at later timepoints ( $>7$ days) when memory loss has been shown to occur in other septic studies [33, 34]. Taken together, the data discussed in Chapter 2 of this dissertation provided a novel role for TNAP activity in cerebral microvessels (Fig. 1). Despite these advancements in understanding the role of TNAP activity in cerebral microvessels, it remains unclear what parts of the brain's vascular tree (arteries, capillaries, or veins) may be critical for BBB maintenance relating to TNAP function.

The cerebral vasculature is heterogenous and displays an arteriovenous hierarchy similar to peripheral vascular beds. Moreover, the expression of a single protein or molecule differs across the cerebral arteriovenous vascular bed. For example, a recent study demonstrated that the expression of von Willebrand Factor (VWF) is increased in venous BMECs compared to brain arterial and capillary BMECs, whereas, major facilitator super family domain containing 2a (Mfsd2a) protein is highly abundant in capillary BMECs compared to brain arterial and venous BMECs [35]. The localization of TNAP activity in the brain endothelium has been explored in an earlier study. Vorbrodt et al. showed that adult mouse capillary and arterial BMECs express strong TNAP activity, however, venules showed a complete lack of TNAP 
activity. Importantly, despite brain arterioles and capillaries sharing a strong TNAP activity expression, electron microscopy revealed that capillaries express TNAP activity on the luminal surface, whereas, arterioles express TNAP activity on the luminal and abluminal surfaces of BMECs [36]. Intriguingly, a recent study utilizing single-cell RNA sequencing demonstrated that TNAP protein (i.e., Alp/) is also present in the cerebral venous endothelium. Furthermore, this same study also demonstrated that $A / p /$ is highly abundant in brain endothelial cells compared to astrocytes, pericytes, neuron, microglia, and oligodendrocytes [17]. It is unclear whether this new finding contradicts the earlier findings from the Vorbrodt et al. study, which showed an absence of TNAP activity on the venous endothelium using electron microscopy, or rather demonstrates that the protein is present on the venous endothelium but its activity is absent. Spatial/regional brain microvascular expression of TNAP activity was examined in this dissertation. We showed that TNAP activity is highly expressed in striatum (highest TNAP expression) and cortex compared to the hippocampal cerebral microvessels [16].

Future studies, will need to utilize multiphoton imaging, optical tissue clearing, and electron microscopy methods to examine TNAP brain zonation in arterioles, venules, and capillaries given that these vascular regions are morphologically and functionally different [37]. For example, arteriolar vessels have been shown to regulate blood-flow in the cerebral microvasculature via smooth muscle actin, and this regulation is known as neurovascular coupling $[38,39]$. On the other hand, brain capillaries are importance sites of molecular and nutrient transport [37], whereas, post-capillary venules have been shown to play an important role in immune cell transmigration [40]. It remains unclear how a loss of BMEC TNAP may affect the function of these different vessel types in health and disease. The availability of the VE-cKO mice along with specific pharmacological inhibitors (TNAPi and SBI-425) could help answer these new questions. For example, VE-cKO mice would be injected via the retroorbital vein with LysM green fluorescent protein (GFP) monocytes or CD4-GFP T-cells, thereafter, multiphoton in vivo live imaging could be used to examine immune cell extravasation at the post-capillary 
venules in naïve VE-cKO and septic VE-cKO mice. Alternatively, flow cytometric methods could be used to assess whole brain immune cell populations in naïve VE-cKO and septic VE-cKO mice.

\section{TNAP-mediated signaling pathways in brain endothelium: a mechanism to explain the repercussions of diminished cerebral microvascular TNAP activity}

Alkaline phosphatases such as TNAP have been previously shown to detoxify toxic molecules such as lipopolysaccharide (LPS) [41]. Other known substrate for TNAP under healthy conditions include: inorganic pyrophosphates (PPi), vitamin B6, adenosine triphosphate (ATP), adenosine diphosphate (ADP), adenosine monophosphate (AMP), and phosphoethanolamine (PEA) [42, 43]. Despite the intriguing findings of a loss of brain microvascular TNAP coupled to BBB dysfunction in sepsis as shown in Chapter 2, it was not apparent on what factors or molecules induced brain microvascular TNAP loss following sepsis, and whether this loss could be seen in other acute neuroinflammatory conditions such as stroke. These new questions were answered in Chapter 3, which showed that the loss of TNAP activity in cerebral microvessels during sepsis manifests similarly in the cortical and striatal penumbra following transient ischemic stroke in mice [16]. Sepsis and stroke animal models have been shown to exhibit a rapid increase in systemic and cerebral pro-inflammatory cytokines [31, 44-46], therefore, we hypothesized that the presence of pro-inflammatory cytokines would decrease BMEC TNAP activity. As expected, treatment of hCMEC/D3 with TNF- $\alpha$ and interferon-gamma (IFN- $\gamma$ ) significantly decreased BMEC TNAP activity to similar levels as the use of TNAPi. More importantly, we showed that the decrease in TNAP activity was coupled to a loss of paracellular barrier integrity. Thereafter, we delineated the mechanisms through which the loss of paracellular barrier integrity occurred in hCMEC/D3 cells.

BMEC TNAP was studied in this dissertation as opposed to other BBB cell types because BMECs have been shown to express abundant TNAP protein compared to astrocytes 
and pericytes [17]. In the brain, the molecular mechanisms through which TNAP operates are well studied in neurons but not well elucidated in brain endothelial cells. For an in-depth discussion on neuronal TNAP function, the reader is directed towards these excellent reviews [47-49]. Preliminary evidence from the Deracinois et al. group was the first study to show that inhibition of TNAP using a pan-AP inhibitor (levamisole) in bovine capillary endothelial cells (BCECs) increased barrier permeability to Lucifer Yellow $\left(\mathrm{Pe}^{\mathrm{LY}}\right)$ [50]. Our data in Chapter 3 corroborates this finding. In Chapter 3, we utilized the Real Time Cell Analysis Dual Plate instrument (RTCA DP) that measures cell impedance as a function of cell viability, cell morphology, and degree of adhesion. The presence of cells in the wells act as an insulator and alters the ionic environment. Therefore, adhesive cells exhibit higher cell impedance values, whereas, non-adhesive cells should theoretically exhibit a lower cell impedance (see RTCA DP instrument manual: http://www.cytometrie-imagerie-saint antoine.org/media/4046/RTCA_DP_System_Operator_Manual_v3.pdf). Pharmacological inhibition of TNAP with a specific TNAP inhibitor (TNAPi) in an appropriate human BBB endothelial cell line model (hCMEC/D3 cells) demonstrated worsened paracellular barrier impedance. Furthermore, primary BMECs (pBMECs) cultured from 6-8 weeks old mice with a conditional deletion of endothelial TNAP (VE-cKO) demonstrated a worsened paracellular barrier impedance. Together, these results suggest that BMEC TNAP activity plays an important role in maintaining barrier integrity, however, the mechanisms through which this occurred remained unclear.

Next, we showed that the loss in barrier impedance resulted from cytoskeletal remodeling denoted by cellular detachment and loss of fluorescence intensity of actin and vimentin. Rho-associated protein kinase [51] proteins belong to a family of serine/threonine kinases and are often downstream targets of Rho GTPases. Following activation, ROCK inhibits the phosphorylation of myosin II regulatory light chain (MLC) and MLC phosphatase (MLCP) which are both important for cellular relaxation, inhibition of stress fiber formation, and 
prevention of cell-cell detachment [52]. More importantly, increased ROCK protein expression has been implicated in many neurodegenerative diseases including amyotrophic lateral sclerosis [53], Huntington's disease (HD), AD, and Parkinson's disease (PD) [54]. Thus, we explored the role of the ROCK pathway in mediating cytoskeletal remodeling following TNAPi treatment. Our results showed that TNAPi increased BMEC expression of ROCK 1 and 2 proteins. Interestingly, TNAPi did not significantly increase Ras homolog family member A (RhoA; an Upstream target of ROCK) protein expression. This indicates that TNAP activity may decrease ROCK expression through another pathway that is independent of RhoA. Future studies will need to examine what additional proteins are involved in TNAP-induced downregulation of ROCK protein expression.

In a final set of experiments, we utilized a pan-ROCK inhibitor to demonstrate that ROCK inhibition mitigated the worsened barrier integrity seen in TNAPi-treated hCMEC/D3 cells or VE-cKO pBMECs [16]. These results elucidated a novel mechanism through which TNAP is able to maintain barrier integrity in brain endothelial cells (Fig. 2A-C), and demonstrates why drugs like fasudil have shown some therapeutic efficacy in preclinical models of stroke [55-57]. For example, Fukuta et al. showed that co-administration of fasudil liposomes and tissue plasminogen activator (t-PA) significantly decreased brain endothelial damage and improved motor scores [56]. However, there is a paucity of data on the utility of fasudil to improve sepsis associated mortality and neurological impairment. Pilot data from our laboratory suggest that fasudil treatment alone for 6 days following sepsis initiation decreased microglia activation (amoeboid morphology; see arrows) and astrocyte proliferation in the dentate gyrus of the hippocampus in mice (Fig. 2D). Future studies will address whether fasudil alone or combined treatment of fasudil and AP-based drugs is able to improve survival, BBB dysfunction, and neurological impairment post-sepsis.

\section{Deciphering the role of BMEC TNAP at the neurovascular unit in vivo}




\section{A. A Potential role for TNAP in the regulation of BBB permeability}

In Chapter 4, we sought to elucidate the role of BMEC TNAP in vivo given that our in vitro and ex vivo data shown in Chapter 3 supported a role for BMEC in maintaining paracellular integrity. Furthermore, the data in Chapter 4 expanded on the limitations of the study conducted in Chapter 3. We showed that naïve VE-cKO mice demonstrated a whole brain size selective permeability to small molecules ( $380 \mathrm{Da})$. Interestingly, we did not observe any differences in spatial permeability between naïve VE-cKO and $A / p f^{f / f t}$ mice. Three plausible explanation may be responsible for this finding: 1) The tissues collected for quantification were not sufficient enough to observe the size selective permeability we saw in the whole brain permeability study 2) The dyes used were sensitive to the tissue processing procedure and 3) The imaging modality was not sensitive enough to detect the size selective permeability in tissue sections. To address this issues, ongoing studies will utilize multiphoton live animal imaging to elucidate spatial permeability in naïve VE-cKO mice. Alpl (TNAP protein) has been shown in a recent study to regulate caveolin mediated transcytosis (transcellular transport) [17]. Future studies utilizing electron microscopy, Mfsd2a (a marker for transcytosis [58]) immunohistochemistry, and albumin permeability assays will address the role of BMEC TNAP in regulating transcellular barrier integrity.

Next, we explored whether the size selective permeability seen in naïve VE-cKO mice was worsened in sepsis. Our data demonstrated that VE-cKO mice were more susceptible to significant BBB dysfunction $24 \mathrm{~h}$ post-sepsis compared to Alp $\mathrm{fl}^{f / f t}$ septic mice. Despite this intriguing finding, we had expected that VE-cKO mice should have exhibited a remarkable loss of barrier integrity in normal physiology and sepsis, given the data derived from Chapters 2 and 3. Upon examining the literature, we found that other cell types that comprise the BBB such as astrocyte end-feet processes and pericytes also expressed TNAP activity [59-61]. Furthermore, co-culture of BMECs with mixed glial cells in vitro has been shown to drastically increase TNAP protein, activity, and mRNA expression in BMECs compared to solo-cultures [50, 62, 63]. In 
another study, Tio et al. used conditioned medium to demonstrate that secreted products from astrocytes were capable of inducing an increase in TNAP activity expression in endothelial cells [64]. Owing to the finding from these studies, we examined whether naïve VE-cKO mice still expressed TNAP activity in cerebral microvessels. We demonstrated in Chapter 3 that pBMEC cultures from VE-cKO mice did not show any TNAP activity compared to controls. Therefore, if TNAP activity remained present in cerebral microvessels of VE-cKO mice, it is likely that this activity originates from other BBB cell types such as astrocytes and pericytes. We showed here that indeed TNAP activity was still present in cerebral microvessels of VE-cKO mice (Fig. 3A). More importantly, cerebral microvascular TNAP activity colocalized with aquaporin-4 (AQP4), a marker for astrocyte end-feet processes (Fig. 3B) [65]. Therefore, it is plausible that the continued presence of TNAP activity in cerebral microvessels may be responsible for the small size selective BBB permeability seen in naïve VE-cKO mice. This suggestion is supported by the Deracinois et al. study which showed that BCECs co-cultured with mixed glial cells did not exhibit increase permeability to $\mathrm{Pe}^{\mathrm{LY}}$ (> $380 \mathrm{Da}$ ) following levamisole (pan-AP inhibitor) treatment [50]. Consequently, future studies will need to address the contributions of these cells with widely available astrocyte and pericyte Cre-driver mouse (Aldh1/1-Cre/ER ${ }^{T 2}$ and PDGFR $\beta$ $P 2 A-C r e E R^{T 2}$ respectively) $[66,67]$. Furthermore, we demonstrated in Chapter 4 that the magnitude of BBB dysfunction in VE-cKO mice may become apparent in severe disease conditions such as stroke. In summary, these studies support a role for TNAP in maintaining BBB integrity in disease.

\section{B. Emerging roles for brain microvascular TNAP in aging, Alzheimer's disease, and Alzheimer's} disease related dementia's (ADRD)

Aging increases susceptibility of brain endothelial cells to injury or toxins [68]. Preclinical studies utilizing plasma substitution therapies from aged mice into young mice have been able to show the significant effects of aging on brain endothelial cell function and zonation $[69,70]$. 
The role for BMEC TNAP in aging is poorly understood, however, recent data suggest that brain microvascular TNAP protein (i.e., Alpl) expression is decreased in the hippocampus of aged mice (20 months old) compared to young mice (3 months) [70]. Given the observation of increased TNAP protein expression with aging, we examined whether brain microvascular TNAP activity also increased with aging. As shown in Figure 4A, TNAP activity increased in cerebral microvessels of the cortex, striatum, and hippocampus (CA3 shown) in 14-month-old mice (middle aged) compared to 4-month-old mice (young mice). These results suggest a dichotomy in brain microvascular TNAP function whereby following injury TNAP activity is diminished while in aging TNAP activity increases in cerebral microvessels. The implications of the increased brain microvascular TNAP protein expression were further explored in a new report which showed that inhibition of brain microvascular TNAP in aged mice enhanced transcytosis of plasma proteins into the brain parenchyma [17]. To our knowledge, these are the only studies that have investigated a role of brain microvascular TNAP in aging. Notably, a recent transcriptome study demonstrating that each vessel segment (i.e., arteries, capillaries, and venules) age differently emphasizes the need to consider differential functions of the vascular tree when studying BMEC TNAP function [70]. Likewise, the role of sex-specific BMEC TNAP function in aging remains to be elucidated.

Aging is a risk factor for dementia and AD. However, no studies have examined the role of BMEC TNAP or cerebral microvascular TNAP in AD pathogenesis and progression. We present here for the first time preliminary evidence suggesting that brain microvascular TNAP is decreased in a model of AD (APPSwDI/Nos2 ${ }^{-/-}$or CVN-AD [71]) compared to wild-type mice (Figure 4B). This finding contrast neuronal TNAP studies which demonstrated that neuronal TNAP exacerbates tau toxicity in AD through its dephosphorylation mechanism [72]. We had previously shown that 8-month-old female CVN-AD mice displayed AD associated neuropathology which coupled sleep, metabolic, and sensorimotor dysfunction [73]. Female mice were used due to a higher prevalence of $A D$ in females than males [74]. In an unpublished 
study, we examined whether sepsis would exacerbate AD progression in 8-month-old CVN-AD female mice. Our results showed that sepsis worsened memory retention on the radial arm water maze (RAWM). Interestingly, memory loss in AD septic mice coupled to IgG infiltration and loss of cerebral microvascular TNAP activity (Figure 4C). These findings suggest a role for TNAP in AD pathogenesis. Future studies are needed to assess the role of cerebral microvascular TNAP in AD pathogenesis and progression. Experimental paradigms that involve chronic treatment of AD animals with TNAPi prior to the development of AD neuropathology would help bridge this knowledge gap. Given the preliminary data discussed here, we speculate that AD animals treated with TNAPi would demonstrate worsened AD neuropathology and cognitive outcomes. Data from this dissertation as described in Chapter 3 supports the use of fasudil alone or combined treatment of fasudil and AP-based drugs as a therapy for AD. This suggestion is corroborated by a recent study showing that AD mice treated with fasudil exhibited decreased neuroinflammation (activation of microglia and astrocytes) and improved memory and learning [75].

\section{Restoring AP activity as therapy to treat neuroinflammatory diseases}

To date, there are few pharmaceuticals available for exogenous treatment to restore AP activity. However, some AP-based therapeutic agents have been investigated in both preclinical and clinical studies. Recombinant human TNAP (rhTNAP) purified from rabbit transgenic milk samples and administered to LPS-infected mice resulted in increased survival [76]. Clinical trials using bovine intestinal AP (bIAP) have shown functional improvement in patients with sepsis and ulcerative colitis [77-80]. However, due to bIAP side effects, a human recombinant AP (recAP), which encompasses the stability afforded from placental AP and catalytic viability of IAP, was created and has been promising in preclinical trials for treatment of sepsis-induced acute kidney injury (sepsis-AKI) [81, 82]. One drawback is that recAP administration did not affect pulmonary inflammation or endothelial and epithelial dysfunction in rats [83], and it failed 
to improve short-term kidney function in patients with sepsis-associated acute kidney injury [84]. This compound is currently in a Phase III clinical trials that has enrolled patients in European and North American hospitals. The data in this dissertation provide preclinical evidence to support the use of rhTNAP or recAP to treat sepsis and stroke patients. Future studies will need to assess whether rhTNAP or recAP are able to mitigate BBB dysfunction and long-term cognitive impairment seen in sepsis survivors.

\section{A plausible role for the hypothalamic-pituitary-adrenal axis (HPA) in regulating endothelial TNAP activity}

\section{A. The HPA axis}

The HPA axis collectively comprises the organs and secretions whose activation results in the release of corticosteroid (CORT) hormones from the adrenal glands. Physiological actions of CORT are primarily regulatory and are involved in the establishment and maintenance of homeostasis. At the cellular level, corticosteroids interact with two types of steroid hormone receptors: mineralocorticoid receptors (MR), which bind CORT and aldosterone (ALD), and glucocorticoid receptors (GR), which bind CORT [85, 86]. MR has a 10-fold higher affinity for CORT than GR resulting in high receptor occupation at normally low circulating levels of hormone [87]. GR occupancy increases as a result of elevated levels of hormone during stress, and the peaks of fluctuating hormone levels linked to circadian or ultradian rhythms [88]. This occupancy profile suggests the majority of corticosteroid-regulated effects are mediated through GR. CORT modulates a number of systems and processes such as immunity, inflammation, metabolism, and energy balance in a variety of cell types [89]. Importantly, CORT has been shown to regulate the stability of the BBB [90]. In the brain, MR expression is restricted to the neurons of limbic brain regions including the hippocampus, lateral septum, amygdala, and prefrontal cortex [91]. GR expression is more ubiquitous among cells in all tissue types, and especially in neurons, endothelial cells, and glia of the brain [92]. 


\section{B. Glucocorticoid regulation of the BBB in health and systemic inflammation}

Glucocorticoids (GCs) are a class of CORT with strong anti-inflammatory properties [93]. Experimental studies have shown an upregulation of TJ proteins, increased electrical resistance, and reduction in vascular permeability following treatment with synthetic GCs [9496]. GC treatment in pathology and physiological conditions has been shown to reduce leukocyte infiltration, enhance transendothelial electrical resistance (TEER), and increase the expression of efflux transporters [97-99]. Administration of GC inhibits the translocation of brain endothelial pro-inflammatory signals like NF-k $\beta$ and reduces the binding of GATA and AP-1 to DNA elements [100]. The summative effect of blocking NF-k $\beta$ endothelial translocation and other pro-inflammatory signals via GC treatment is a reduction in several inflammatory cytokines and chemokines (IL-6, CXCL8, CCL2, etc.), decreased immune cell trafficking across the BBB, increased MMP inhibitors (TIMP-1, TIMP-3, etc.), and downregulation of endothelial integrins (ICAM-1, VCAM-1, etc.) [101-105].

The TJ protein, occludin, is important to the electrical resistance of the junction and contributes to the formation of aqueous pores within the junction [106]. Occludin transcription is positively regulated by glucocorticoid response elements (GREs) [107]. Claudin is another TJ protein regulated by GCs. The claudin family consists of at least 24 conserved proteins, several of which play a role in maintenance of the BBB, including claudin-1, $-3,-5$, and -12 [108-110]. The 5' promoter region of claudin-5 has been shown to contain several putative GREs that confer increased claudin-5 transcription following GC treatment $[111,112]$.

GCs also have direct effects on enzymes localized to the endothelium such as TNAP. Adams and Melnykovych showed that bovine aortic endothelial cells treated with dexamethasone demonstrated an elevated increase in TNAP activity [113]. Despite these intriguing results, it is unclear till date whether these findings extrapolate to BMECs. We speculate that the BMECs treated with dexamethasone would demonstrate an increase in TNAP activity that is coupled to an increase in barrier impedance (integrity). This speculation is 
not unwarranted since several studies have shown that dexamethasone improved barrier integrity in several models [114].

More importantly, other molecules such as retinol, cyclic AMP (cAMP), transforming growth factor-beta (TGF- $\beta$ ), interleukin-6 (IL-6), and basic fibroblast growth factor (bFGF) are known to modulate TNAP activity [13]. While retinol, cyclic cAMP, IL-6, and bFGF have been shown in previous studies to increase TNAP activity $[113,115-117]$, TGF- $\beta$ has been shown to suppress TNAP activity in brain ECs [117]. The mechanisms through which GCs and the aforementioned molecules modulate TNAP activity remain to be elucidated. Likewise, it remains unclear how the presence of TNAP activity on BMECs influences targeted downstream pathways associated with these molecules.

\section{Concluding remarks}

The results discussed in this dissertation demonstrate a novel role for TNAP in maintaining BBB integrity and adds to the only existing scientific literature which had previously examined TNAP's role in maintaining barrier function [13]. Furthermore, the contributions from our study highlight the emerging importance of TNAP in other neuroinflammatory and neurodegenerative diseases. Most importantly, our study lends support for TNAP as a therapeutic target to mitigate the long-term cognitive impairment symptoms seen in septic, stroke and AD patients via the maintenance of BBB integrity. Conversely, TNAP activity could be manipulated to allow pharmacological therapies to penetrate the brain and target cancerous cells. Nevertheless, we must stress that much research is needed to fully elucidate additional mechanisms and signaling pathways through which TNAP is able to maintain barrier integrity. Additional studies are also needed to understand how other TNAP expressing cell types communicate with brain endothelial cells to preserve BBB integrity in the face of injury. 


\section{Figures}

\section{Figure 1}

\begin{tabular}{|c|c|}
\hline \multicolumn{2}{|l|}{ KEY: } \\
\hline & Monocytes \\
\hline & Lymphocytes \\
\hline & Neutrophil \\
\hline & Astrocyte end-feet \\
\hline & Astrocyte \\
\hline & Reactive astrocyte \\
\hline & Surveillance microglia \\
\hline & Activated microglia \\
\hline s & Junctional protein \\
\hline$\therefore$ & Endothelium: TNAP activity \\
\hline$\infty$ & Endothelium: TNAP activity \\
\hline 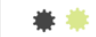 & Pro-inflammatory cytokines \\
\hline
\end{tabular}

(A) Healthy BBB

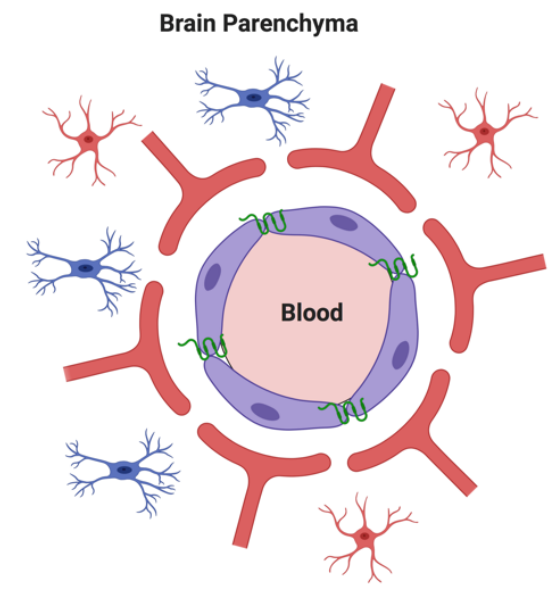

(B) Cortex 7 days post-sepsis

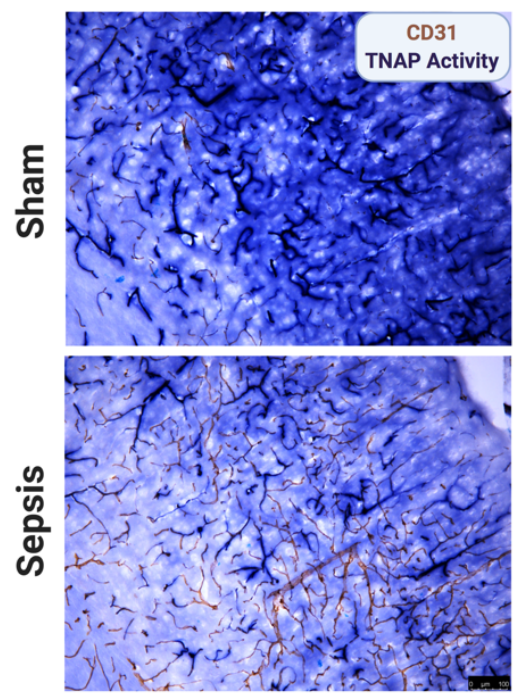

(C) Septic BBB

Astrogliosis

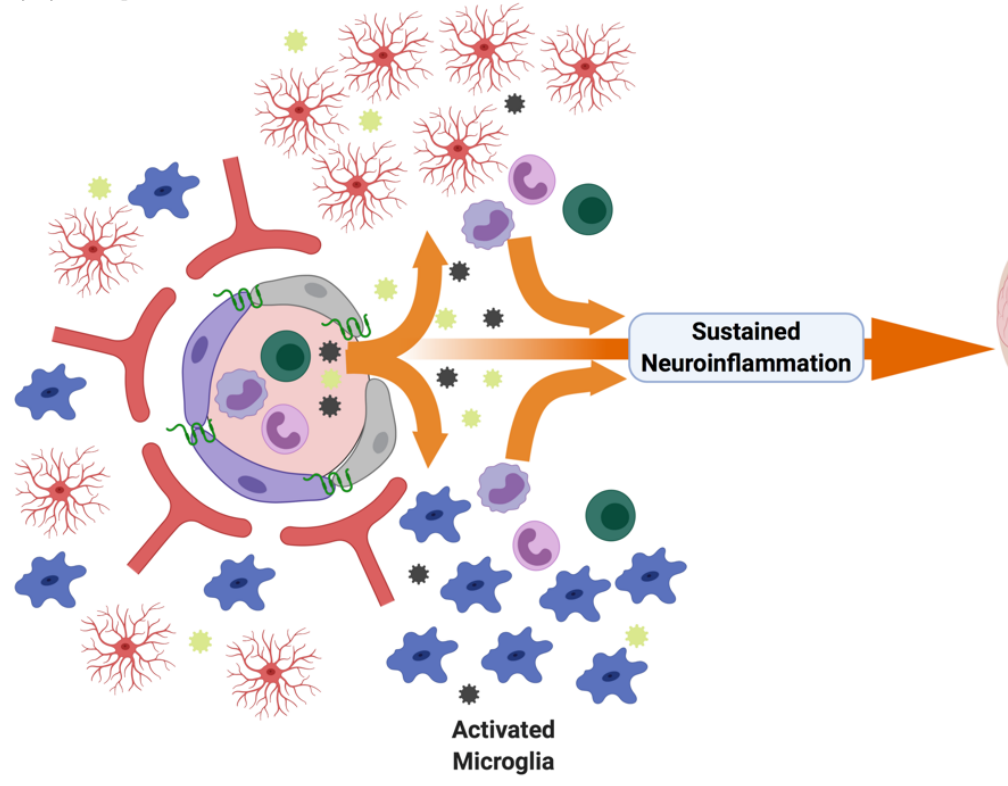

Long-term cognitive impairment

Sensorimotor dysfunction

Increased mortality and morbidity 
Figure 2
(a)
(b)
(c)

\begin{tabular}{c|c:c}
$\begin{array}{c}\text { Health: TNAP enriched } \\
\text { endothelium }\end{array}$ & $\begin{array}{c}\text { Inflammation: TNAP } \\
\text { deficient endothelium }\end{array}$ & $\begin{array}{c}\text { Inflammation: TNAP } \\
\text { deficient endothelium }\end{array}$
\end{tabular}

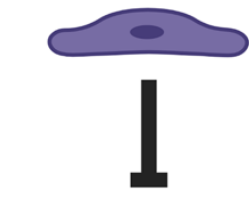

$\downarrow \downarrow$ ROCK $1 / 2$

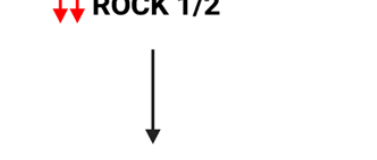

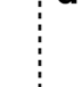
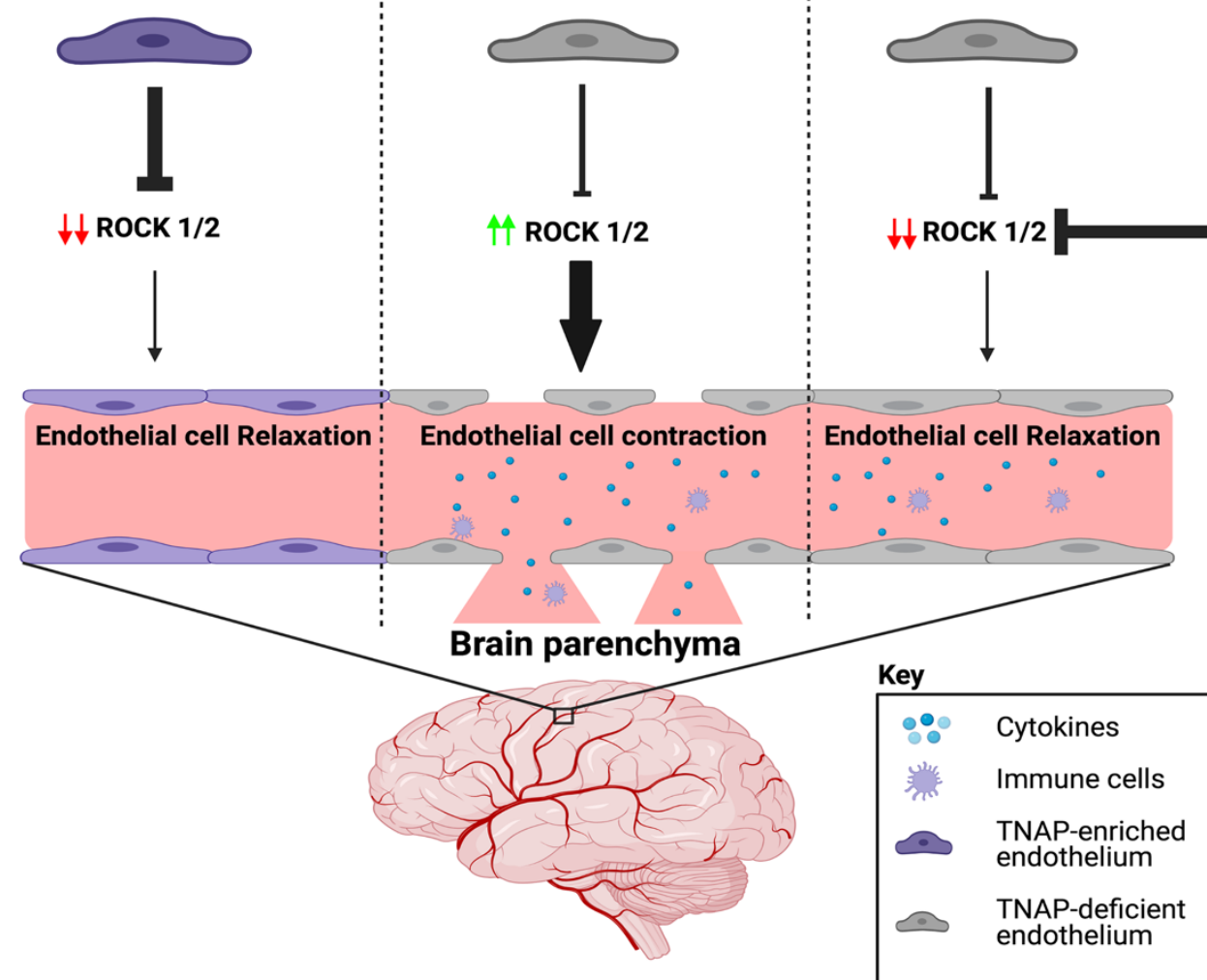

d.
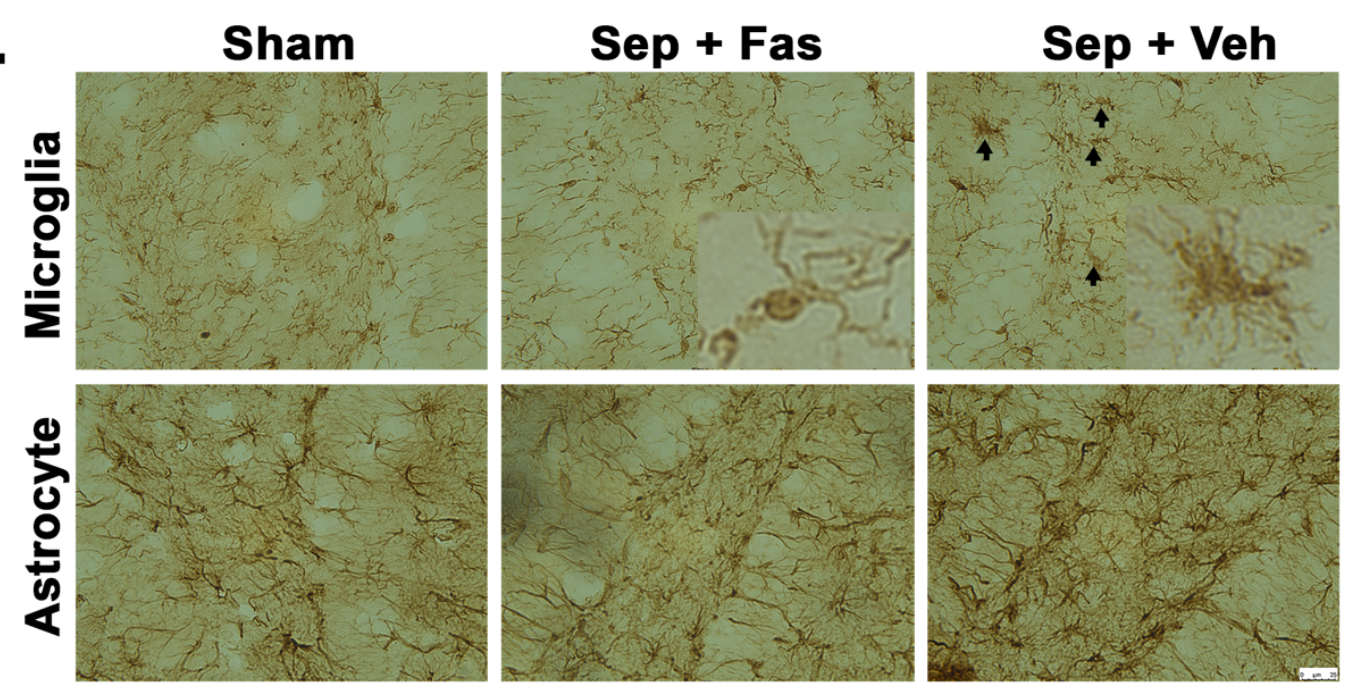
Figure 3
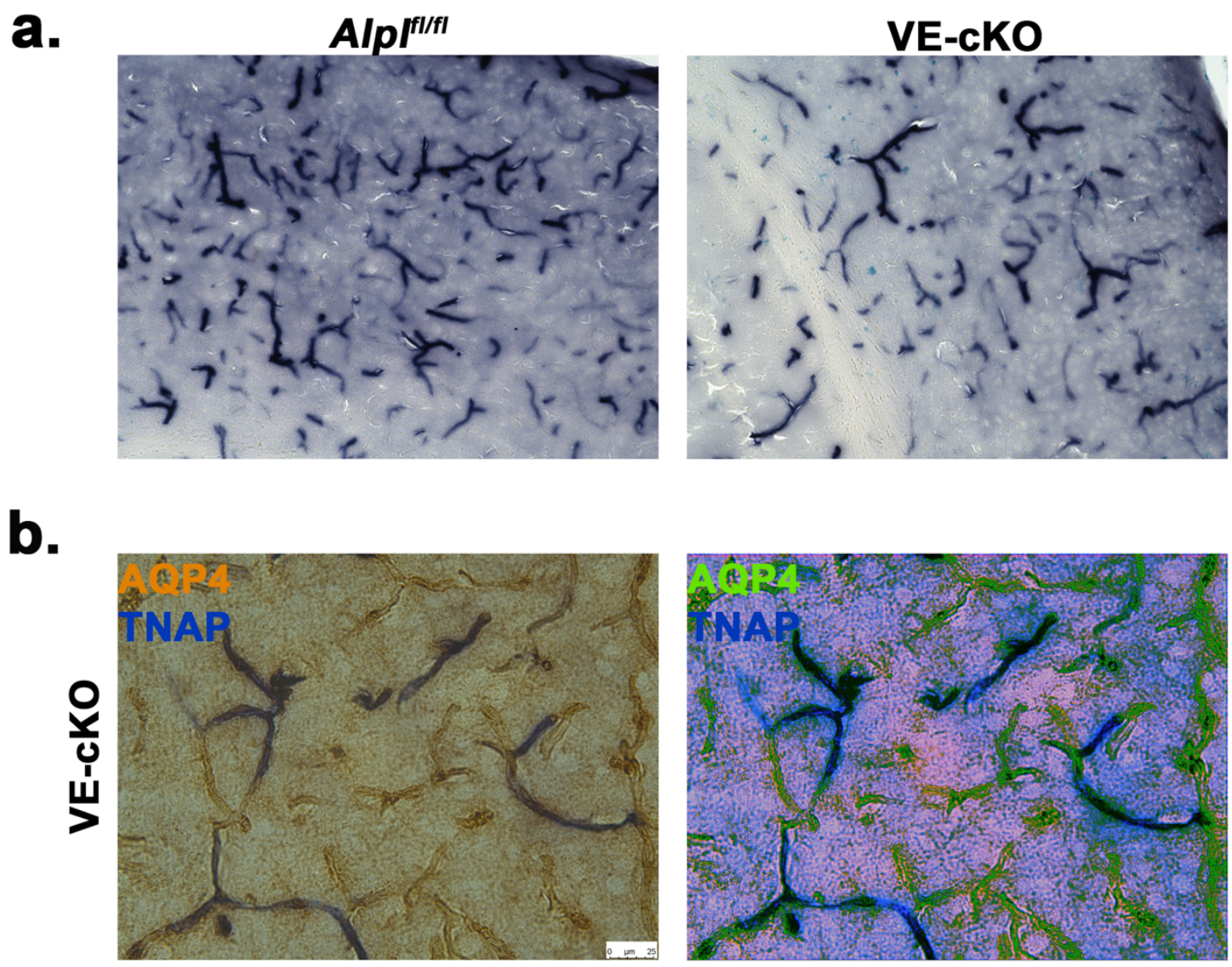
Figure 4
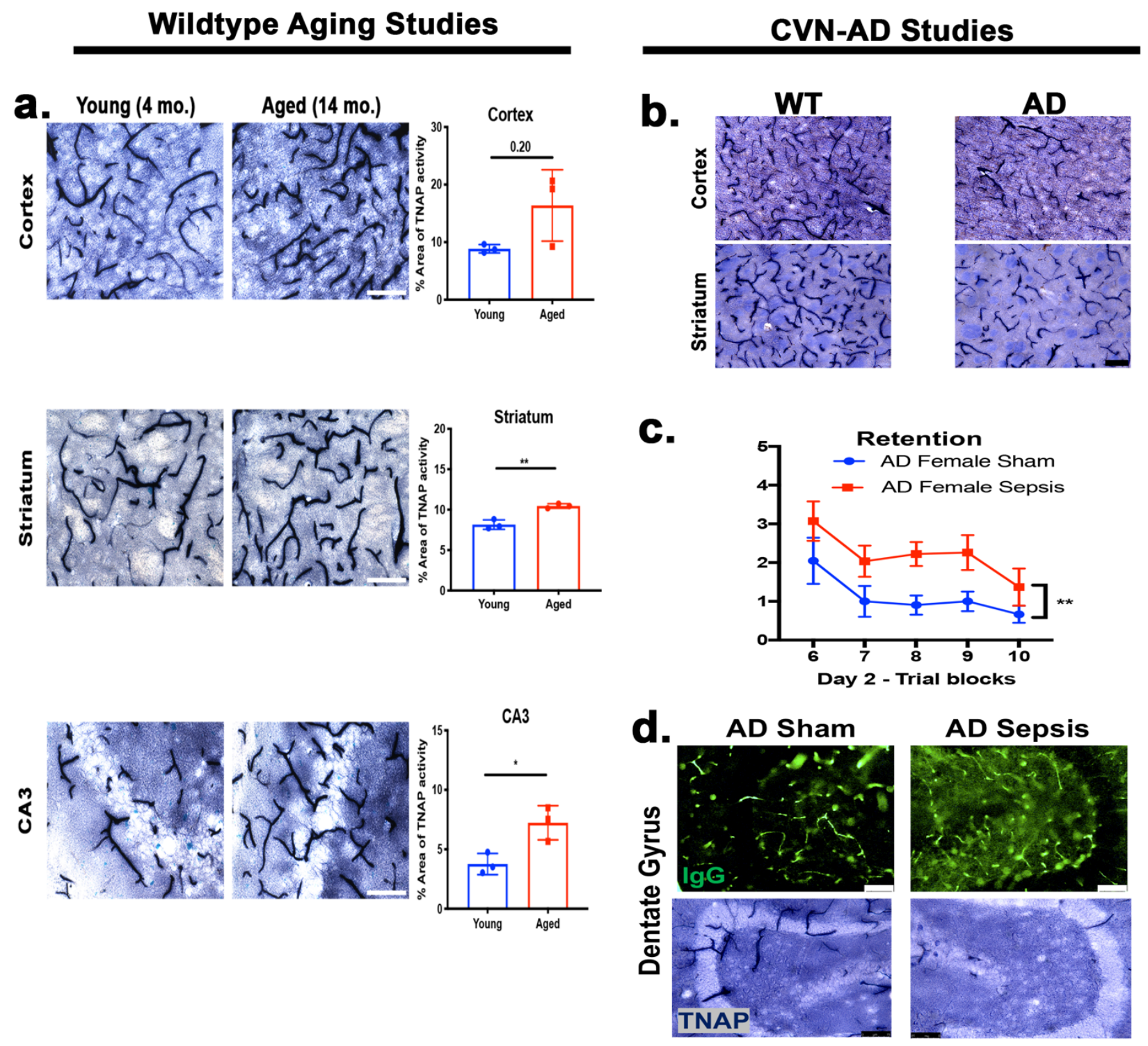


\section{Figure Legends}

Figure 1: Schema for TNAP's role at the BBB during acute systemic inflammation. (A) Under physiologic conditions, the BMEC is enriched with TNAP activity (shown in purple), and this activity is required to maintain precise cerebral homeostasis. (B) Key brain regions (cortex depicted) become susceptible to loss of TNAP activity (i.e., purple stain) in septic compared to sham mice. The loss of TNAP activity on CD31+ (brown) cerebral microvessels is sustained up to 7 days post-sepsis. (C) Following a loss of TNAP activity in BMECs, junctional protein disruption occurs and promotes the infiltration of peripheral pro-inflammatory cytokines and leukocytes into the brain parenchyma. Infiltrating cytokines increase the number of activated microglia and reactive astrocytes. Activated microglia and reactive astrocytes contribute to a state of sustained neuroinflammation which promotes long-term cognitive impairment, sensorimotor dysfunction, and increased mortality and morbidity. BMECs: brain microvascular endothelial cells; TNAP: tissue-nonspecific alkaline phosphatase; BBB: blood-brain barrier. Images were taken at 10X magnification, scale bar $=100 \mu \mathrm{m}$. Image credit: Biorender

Figure 2: ROCK proteins in BMEC TNAP function and fasudil utility as therapeutic for sepsis. (A) In health, BMECs are enriched with TNAP activity. We propose that abundant BMEC TNAP activity decreases ROCK (1/2) protein expression, which maintains the endothelial cytoskeleton by ensuring that brain endothelial cells are in a relaxed state. (B) However, during inflammation, BMEC TNAP activity decreases substantially, and this decrease promotes increased ROCK (1/2) protein expression. Increased ROCK (1/2) protein expression leads to BMEC cytoskeletal contraction and ultimately alters paracellular barrier integrity. Disruption of the BMEC paracellular barrier allows for the infiltration of cytokines and immune cells into the brain parenchyma. (C) Therapeutic injection of fasudil (ROCK inhibitor) during inflammation decreases ROCK (1/2) protein expression, thereby allowing for a maintenance of paracellular barrier integrity despite the loss of BMEC TNAP activity. (D) Mice were injected with fasudil or vehicle for 6 days post-sepsis. On day 8 , mice were euthanized and brains collected for assessment of neuroinflammation using Iba-1 (microglia marker) and GFAP (astrocyte marker). Microglia activation (amoeboid morphology; see arrows) is increased in the dentate gyrus of septic mice treated with vehicle (sep + veh) compared to septic mice treated with fasudil (sep + fas) or sham mice. Astrocyte proliferation is also increased in the dentate gyrus of septic mice treated with vehicle compared to septic mice treated with fasudil or sham mice. BMEC: Brain microvascular endothelial cells; TNAP: tissue-nonspecific alkaline phosphatase; ROCK: rhoassociated protein kinase; Iba-1: ionized calcium binding adaptor molecule 1; GFAP: glial fibrillary acidic protein. Images were taken at $40 \mathrm{X}$ magnification, scale bar $=75 \mu \mathrm{m}$. Image credit: Biorender

Figure 3: Astrocyte end-feet and cerebral microvascular TNAP activity colocalization. (A) Despite a knockout of BMEC TNAP in cerebral microvessels, TNAP activity is still present in naïve VE-cKO mice. Scale bar $=75 \mu \mathrm{m}(B)$ Bright field image and 3D reconstruction showed that astrocyte end-feet processes visualized with AQP4 (green; 3D resconstruction) colocalized (cyan; 3D reconstruction) with TNAP activity in cerebral microvessels (purple). Scale bar $=25$ $\mu \mathrm{m}$. TNAP: Tissue-nonspecific alkaline phosphatase; 3D: 3-dimension.

Figure 5: Brain microvascular TNAP activity in aging and in a mouse model of AD. (A) The striatum $(p=0.004)$ and hippocampal CA3 $(p=0.02)$ regions revealed a significant increase in brain microvascular TNAP activity in aged male mice (14 months old) compared to young male mice (4 months old). The cortex also revealed an increase in TNAP activity in aged mice, however, this increase was not statistically significant $(p=0.20)$. Images taken at $20 \mathrm{X}$ magnification and scale bar $=100 \mu \mathrm{m}$. (B) Preliminary data from male CVN-AD mice showed a 
decrease in cerebral microvascular TNAP activity in the cortex and striatum compared to WT (C57BL/6J) mice. (C) Female AD sepsis mice exhibited loss of memory in the RAWM retention test (D) Female AD sepsis mice also exhibited increased microvascular infiltration of IgG which was coupled to a loss of brain microvascular TNAP activity. Images taken at 20X magnification and scale bar $=75 \mu \mathrm{m}$. TNAP: Tissue-nonspecific alkaline phosphatase; AD: Alzheimer's disease; WT: Wild-type; and RAWM: radial-arm water maze. ${ }^{*}$ indicates $p<0.05$ and ${ }^{* *} p<0.01$. 


\section{References}

1. Yu, Q.J., et al., Targeting brain microvascular endothelial cells: a therapeutic approach to neuroprotection against stroke. Neural Regen Res, 2015. 10(11): p. 1882-91.

2. Engelhardt, B. and S. Liebner, Novel insights into the development and maintenance of the blood-brain barrier. Cell Tissue Res, 2014. 355(3): p. 687-99.

3. Hollenbeck, P.J., et al., Intermediate filament collapse is an ATP-dependent and actindependent process. J Cell Sci, 1989. 92 ( Pt 4): p. 621-31.

4. Kniesel, U. and $\mathrm{H}$. Wolburg, Tight junctions of the blood-brain barrier. Cell Mol Neurobiol, 2000. 20(1): p. 57-76.

5. Lochhead, J.J., et al., Structure, Function, and Regulation of the Blood-Brain Barrier Tight Junction in Central Nervous System Disorders. Front Physiol, 2020. 11: p. 914.

6. Crone, C. and S.P. Olesen, Electrical resistance of brain microvascular endothelium. Brain Res, 1982. 241(1): p. 49-55.

7. Cramer, S.P., et al., Abnormal blood-brain barrier permeability in normal appearing white matter in multiple sclerosis investigated by MRI. Neuroimage Clin, 2014. 4: p. 182-9.

8. Cramer, S.P., et al., Permeability of the blood-brain barrier predicts no evidence of disease activity at 2 years after natalizumab or fingolimod treatment in relapsingremitting multiple sclerosis. Ann Neurol, 2018. 83(5): p. 902-914.

9. van de Haar, H.J., et al., Blood-Brain Barrier Leakage in Patients with Early Alzheimer Disease. Radiology, 2016. 281(2): p. 527-535.

10. Montagne, A., et al., Blood-brain barrier breakdown in the aging human hippocampus. Neuron, 2015. 85(2): p. 296-302.

11. Nation, D.A., et al., Blood-brain barrier breakdown is an early biomarker of human cognitive dysfunction. Nat Med, 2019. 25(2): p. 270-276.

12. Verheggen, I.C.M., et al., Imaging the role of blood-brain barrier disruption in normal cognitive ageing. Geroscience, 2020. 42(6): p. 1751-1764.

13. Deracinois, B., et al., Tissue Non-specific Alkaline Phosphatase (TNAP) in Vessels of the Brain. Subcell Biochem, 2015. 76: p. 125-51.

14. Brichacek, A.L., et al., Systemic inhibition of tissue-nonspecific alkaline phosphatase alters the brain-immune axis in experimental sepsis. Sci Rep, 2019. 9(1): p. 18788.

15. Nwafor, D.C., et al., Loss of tissue-nonspecific alkaline phosphatase (TNAP) enzyme activity in cerebral microvessels is coupled to persistent neuroinflammation and behavioral deficits in late sepsis. Brain Behav Immun, 2020. 84: p. 115-131.

16. Nwafor, D.C., et al., Brain endothelial cell tissue-nonspecific alkaline phosphatase (TNAP) activity promotes maintenance of barrier integrity via the ROCK pathway. 2021: bioRxiv.

17. Yang, A.C., et al., Physiological blood-brain transport is impaired with age by a shift in transcytosis. Nature, 2020. 583(7816): p. 425-430.

18. Liu, J., et al., Tissue-nonspecific alkaline phosphatase deficiency causes abnormal craniofacial bone development in the Alpl(-/-) mouse model of infantile hypophosphatasia. Bone, 2014. 67: p. 81-94.

19. Waymire, K.G., et al., Mice lacking tissue non-specific alkaline phosphatase die from seizures due to defective metabolism of vitamin B-6. Nat Genet, 1995. 11(1): p. 45-51.

20. Foster, B.L., et al., Conditional Alpl Ablation Phenocopies Dental Defects of Hypophosphatasia. J Dent Res, 2017. 96(1): p. 81-91.

21. Dahl, R., et al., Discovery and validation of a series of aryl sulfonamides as selective inhibitors of tissue-nonspecific alkaline phosphatase (TNAP). J Med Chem, 2009. 52(21): p. 6919-25.

22. Pinkerton, A.B., et al., Discovery of 5-((5-chloro-2methoxyphenyl)sulfonamido)nicotinamide (SBI-425), a potent and orally bioavailable 
tissue-nonspecific alkaline phosphatase (TNAP) inhibitor. Bioorg Med Chem Lett, 2018. 28(1): p. 31-34.

23. Alva, J.A., et al., VE-Cadherin-Cre-recombinase transgenic mouse: a tool for lineage analysis and gene deletion in endothelial cells. Dev Dyn, 2006. 235(3): p. 759-67.

24. Brown, W.R., et al., Vascular dementia in leukoaraiosis may be a consequence of capillary loss not only in the lesions, but in normal-appearing white matter and cortex as well. J Neurol Sci, 2007. 257(1-2): p. 62-6.

25. Goncharov, N.V., et al., Markers and Biomarkers of Endothelium: When Something Is Rotten in the State. Oxid Med Cell Longev, 2017. 2017: p. 9759735.

26. Romanelli, F., et al., Overexpression of tissue-nonspecific alkaline phosphatase (TNAP) in endothelial cells accelerates coronary artery disease in a mouse model of familial hypercholesterolemia. PLoS One, 2017. 12(10): p. e0186426.

27. Savinov, A.Y., et al., Transgenic Overexpression of Tissue-Nonspecific Alkaline Phosphatase (TNAP) in Vascular Endothelium Results in Generalized Arterial Calcification. J Am Heart Assoc, 2015. 4(12).

28. Opdebeeck, B., et al., Pharmacological TNAP inhibition efficiently inhibits arterial media calcification in a warfarin rat model but deserves careful consideration of potential physiological bone formation/mineralization impairment. Bone, 2020. 137: p. 115392.

29. Goettsch, C., et al., TNAP as a therapeutic target for cardiovascular calcification - a discussion of its pleiotropic functions in the body. Cardiovasc Res, 2020.

30. Nwafor, D.C., et al., Targeting the Blood-Brain Barrier to Prevent Sepsis-Associated Cognitive Impairment. J Cent Nerv Syst Dis, 2019. 11: p. 1179573519840652.

31. Michie, H.R., et al., Detection of circulating tumor necrosis factor after endotoxin administration. N Engl J Med, 1988. 318(23): p. 1481-6.

32. Iwashyna, T.J., et al., Long-term cognitive impairment and functional disability among survivors of severe sepsis. JAMA, 2010. 304(16): p. 1787-94.

33. Andonegui, G., et al., Targeting inflammatory monocytes in sepsis-associated encephalopathy and long-term cognitive impairment. JCI Insight, 2018. 3(9).

34. Chavan, S.S., et al., HMGB1 mediates cognitive impairment in sepsis survivors. Mol Med, 2012. 18: p. 930-7.

35. Vanlandewijck, M., et al., A molecular atlas of cell types and zonation in the brain vasculature. Nature, 2018. 554(7693): p. 475-480.

36. Vorbrodt, A.W., A.S. Lossinsky, and H.M. Wisniewski, Localization of alkaline phosphatase activity in endothelia of developing and mature mouse blood-brain barrier. Dev Neurosci, 1986. 8(1): p. 1-13.

37. Daneman, R. and A. Prat, The blood-brain barrier. Cold Spring Harb Perspect Biol, 2015. 7(1): p. a020412.

38. Shen, Z., et al., An artery-specific fluorescent dye for studying neurovascular coupling. Nat Methods, 2012. 9(3): p. 273-6.

39. Chow, B.W., et al., Caveolae in CNS arterioles mediate neurovascular coupling. Nature, 2020. 579(7797): p. 106-110.

40. Marchetti, L. and B. Engelhardt, Immune cell trafficking across the blood-brain barrier in the absence and presence of neuroinflammation. Vasc Biol, 2020. 2(1): p. H1-H18.

41. Peters, E., et al., Alkaline phosphatase protects against renal inflammation through dephosphorylation of lipopolysaccharide and adenosine triphosphate. Br J Pharmacol, 2015. 172(20): p. 4932-45.

42. Whyte, M.P., et al., Alkaline phosphatase: placental and tissue-nonspecific isoenzymes hydrolyze phosphoethanolamine, inorganic pyrophosphate, and pyridoxal 5'-phosphate. Substrate accumulation in carriers of hypophosphatasia corrects during pregnancy. $\mathrm{J}$ Clin Invest, 1995. 95(4): p. 1440-5. 
43. Say, J.C., et al., Alkaline phosphatase from rat osseous plates: purification and biochemical characterization of a soluble form. Biochim Biophys Acta, 1991. 1074(2): $p$. 256-62.

44. Barone, F.C., et al., Tumor necrosis factor-alpha. A mediator of focal ischemic brain injury. Stroke, 1997. 28(6): p. 1233-44.

45. Romero, C.R., et al., The role of interferon-gamma in the pathogenesis of acute intraabdominal sepsis. J Leukoc Biol, 2010. 88(4): p. 725-35.

46. Yilmaz, G., et al., Role of T lymphocytes and interferon-gamma in ischemic stroke. Circulation, 2006. 113(17): p. 2105-12.

47. Graser, S., D. Liedtke, and F. Jakob, TNAP as a New Player in Chronic Inflammatory Conditions and Metabolism. Int J Mol Sci, 2021. 22(2).

48. Fonta, C., et al., Rediscovering TNAP in the Brain: A Major Role in Regulating the Function and Development of the Cerebral Cortex. Subcell Biochem, 2015. 76: p. 85106.

49. Zimmermann, H. and D. Langer, Tissue-Nonspecific Alkaline Phosphatase in the Developing Brain and in Adult Neurogenesis, in Neuronal Tissue-Nonspecific Alkaline Phosphatase (TNAP), C. Fonta and L. Négyessy, Editors. 2015, Springer Netherlands: Dordrecht. p. 61-84.

50. Deracinois, B., et al., TNAP and EHD1 are over-expressed in bovine brain capillary endothelial cells after the re-induction of blood-brain barrier properties. PLoS One, 2012. 7(10): p. e48428.

51. Kishnani, P.S., et al., Five-year efficacy and safety of asfotase alfa therapy for adults and adolescents with hypophosphatasia. Bone, 2019. 121: p. 149-162.

52. Liu, J., H.Y. Gao, and X.F. Wang, The role of the Rho/ROCK signaling pathway in inhibiting axonal regeneration in the central nervous system. Neural Regen Res, 2015. 10(11): p. 1892-6.

53. Inc., A.P., STRENSIQ. 2020.

54. Koch, J.C., et al., ROCK inhibition in models of neurodegeneration and its potential for clinical translation. Pharmacol Ther, 2018. 189: p. 1-21.

55. Shibuya, M., et al., Effects of fasudil in acute ischemic stroke: results of a prospective placebo-controlled double-blind trial. J Neurol Sci, 2005. 238(1-2): p. 31-9.

56. Fukuta, T., et al., Combination therapy with liposomal neuroprotectants and tissue plasminogen activator for treatment of ischemic stroke. FASEB J, 2017. 31(5): p. 18791890.

57. Liu, K., et al., Role of rho kinase in microvascular damage following cerebral ischemia reperfusion in rats. Int J Mol Sci, 2011. 12(2): p. 1222-31.

58. Ben-Zvi, A., et al., Mfsd2a is critical for the formation and function of the blood-brain barrier. Nature, 2014. 509(7501): p. 507-11.

59. Mayahara, H., et al., The new lead citrate method for the ultracytochemical demonstration of activity of non-specific alkaline phosphatase (orthophosphoric monoester phosphohydrolase). Histochemie, 1967. 11(1): p. 88-96.

60. Mori, S. and M. Nagano, Electron-microscopic cytochemistry of alkaline-phosphatase activity in endothelium, pericytes and oligodendrocytes in the rat brain. Histochemistry, 1985. 82(3): p. 225-31.

61. Ovtscharoff, W., [Ultracytochemical localisation of the alkaline phosphatase in the cerebral cortex of newborn rats (author's transl)]. Histochemie, 1973. 37(1): p. 93-5.

62. Meyer, J., J. Rauh, and H.J. Galla, The susceptibility of cerebral endothelial cells to astroglial induction of blood-brain barrier enzymes depends on their proliferative state. $\mathrm{J}$ Neurochem, 1991. 57(6): p. 1971-7.

63. Rauh, J., et al., Development of an in vitro cell culture system to mimic the blood-brain barrier. Prog Brain Res, 1992. 91: p. 117-21. 
64. Tio, S., M. Deenen, and E. Marani, Astrocyte-mediated induction of alkaline phosphatase activity in human umbilical cord vein endothelium: an in vitro model. Eur J Morphol, 1990. 28(2-4): p. 289-300.

65. Hubbard, J.A., et al., Expression of the Astrocyte Water Channel Aquaporin-4 in the Mouse Brain. ASN Neuro, 2015. 7(5).

66. Cuervo, $\mathrm{H}$., et al., PDGFRbeta-P2A-CreER(T2) mice: a genetic tool to target pericytes in angiogenesis. Angiogenesis, 2017. 20(4): p. 655-662.

67. Srinivasan, R., et al., New Transgenic Mouse Lines for Selectively Targeting Astrocytes and Studying Calcium Signals in Astrocyte Processes In Situ and In Vivo. Neuron, 2016. 92(6): p. 1181-1195.

68. Banks, W.A., et al., Healthy aging and the blood-brain barrier. Vol. 1. 2021: Nature Aging.

69. Yousef, H., et al., Aged blood impairs hippocampal neural precursor activity and activates microglia via brain endothelial cell VCAM1. Nat Med, 2019. 25(6): p. 988-1000.

70. Chen, M.B., et al., Brain Endothelial Cells Are Exquisite Sensors of Age-Related Circulatory Cues. Cell Rep, 2020. 30(13): p. 4418-4432 e4.

71. Colton, C.A., et al., The effects of NOS2 gene deletion on mice expressing mutated human AbetaPP. J Alzheimers Dis, 2008. 15(4): p. 571-87.

72. Diaz-Hernandez, M., et al., Tissue-nonspecific alkaline phosphatase promotes the neurotoxicity effect of extracellular tau. J Biol Chem, 2010. 285(42): p. 32539-48.

73. Nwafor, D.C., et al., Disruption of metabolic, sleep, and sensorimotor functional outcomes in a female transgenic mouse model of Alzheimer's disease. Behav Brain Res, 2020. 398: p. 112983.

74. Hebert, L.E., et al., Alzheimer disease in the United States (2010-2050) estimated using the 2010 census. Neurology, 2013. 80(19): p. 1778-83.

75. Guo, M.F., et al., Fasudil inhibits the activation of microglia and astrocytes of transgenic Alzheimer's disease mice via the downregulation of TLR4/Myd88/NF-kappaB pathway. J Neuroimmunol, 2020. 346: p. 577284.

76. Bender, B., et al., Recombinant human tissue non-specific alkaline phosphatase successfully counteracts lipopolysaccharide induced sepsis in mice. Physiol Res, 2015. 64(5): p. 731-8.

77. Pickkers, P., et al., Alkaline phosphatase for treatment of sepsis-induced acute kidney injury: a prospective randomized double-blind placebo-controlled trial. Crit Care, 2012. 16(1): p. R14.

78. Pickkers, P., et al., Clinical pharmacology of exogenously administered alkaline phosphatase. Eur J Clin Pharmacol, 2009. 65(4): p. 393-402.

79. Heemskerk, S., et al., Alkaline phosphatase treatment improves renal function in severe sepsis or septic shock patients. Crit Care Med, 2009. 37(2): p. 417-23, e1.

80. Lukas, M., et al., Exogenous alkaline phosphatase for the treatment of patients with moderate to severe ulcerative colitis. Inflamm Bowel Dis, 2010. 16(7): p. 1180-6.

81. Engelmann, C., et al., Recombinant Alkaline Phosphatase Prevents Acute on Chronic Liver Failure. Sci Rep, 2020. 10(1): p. 389.

82. Peters, E., et al., Effects of a human recombinant alkaline phosphatase on renal hemodynamics, oxygenation and inflammation in two models of acute kidney injury. Toxicol Appl Pharmacol, 2016. 313: p. 88-96.

83. Juschten, J., et al., Alkaline phosphatase in pulmonary inflammation-a translational study in ventilated critically ill patients and rats. Intensive Care Med Exp, 2020. 8(Suppl 1): p. 46.

84. Pickkers, P., et al., Effect of Human Recombinant Alkaline Phosphatase on 7-Day Creatinine Clearance in Patients With Sepsis-Associated Acute Kidney Injury: A Randomized Clinical Trial. JAMA, 2018. 320(19): p. 1998-2009. 
85. Arriza, J.L., et al., The neuronal mineralocorticoid receptor as a mediator of glucocorticoid response. Neuron, 1988. 1(9): p. 887-900.

86. Hollenberg, S.M., et al., Primary structure and expression of a functional human glucocorticoid receptor cDNA. Nature, 1985. 318(6047): p. 635-41.

87. Funder, J.W., Aldosterone and Mineralocorticoid Receptors-Physiology and Pathophysiology. Int J Mol Sci, 2017. 18(5).

88. Kolbe, I., R. Dumbell, and H. Oster, Circadian Clocks and the Interaction between Stress Axis and Adipose Function. Int J Endocrinol, 2015. 2015: p. 693204.

89. Burford, N.G., N.A. Webster, and D. Cruz-Topete, Hypothalamic-Pituitary-Adrenal Axis Modulation of Glucocorticoids in the Cardiovascular System. Int J Mol Sci, 2017. 18(10).

90. Lucassen, P.J., et al., Regulation of Adult Neurogenesis and Plasticity by (Early) Stress, Glucocorticoids, and Inflammation. Cold Spring Harb Perspect Biol, 2015. 7(9): p. a021303.

91. McEwen, B.S., et al., Putative estrogen and glucocorticoid receptors in the limbic brain. J Steroid Biochem, 1975. 6(6): p. 971-7.

92. Nicolaides, N.C., et al., The human glucocorticoid receptor: molecular basis of biologic function. Steroids, 2010. 75(1): p. 1-12.

93. Zielinska, K.A., et al., Endothelial Response to Glucocorticoids in Inflammatory Diseases. Front Immunol, 2016. 7: p. 592.

94. Buse, P., et al., Glucocorticoid-induced functional polarity of growth factor responsiveness regulates tight junction dynamics in transformed mammary epithelial tumor cells. J Biol Chem, 1995. 270(47): p. 28223-7.

95. Singer, K.L., et al., Relationship of serine/threonine phosphorylation/dephosphorylation signaling to glucocorticoid regulation of tight junction permeability and ZO-1 distribution in nontransformed mammary epithelial cells. J Biol Chem, 1994. 269(23): p. 16108-15.

96. Forster, C., et al., Occludin as direct target for glucocorticoid-induced improvement of blood-brain barrier properties in a murine in vitro system. J Physiol, 2005. 565(Pt 2): p. 475-86.

97. Paul, C. and C. Bolton, Inhibition of blood-brain barrier disruption in experimental allergic encephalomyelitis by short-term therapy with dexamethasone or cyclosporin A. Int J Immunopharmacol, 1995. 17(6): p. 497-503.

98. Bauer, B., et al., Pregnane $X$ receptor up-regulation of P-glycoprotein expression and transport function at the blood-brain barrier. Mol Pharmacol, 2004. 66(3): p. 413-9.

99. Chan, G.N., et al., In vivo induction of P-glycoprotein expression at the mouse bloodbrain barrier: an intracerebral microdialysis study. J Neurochem, 2013. 127(3): p. 34252.

100. Simoncini, T., et al., Estrogens and glucocorticoids inhibit endothelial vascular cell adhesion molecule-1 expression by different transcriptional mechanisms. Circ Res, 2000. 87(1): p. 19-25.

101. Zakkar, M., et al., Dexamethasone arterializes venous endothelial cells by inducing mitogen-activated protein kinase phosphatase-1: a novel antiinflammatory treatment for vein grafts? Circulation, 2011. 123(5): p. 524-32.

102. Shi, J.X., et al., Tristetraprolin is involved in the glucocorticoid-mediated interleukin 8 repression. Int Immunopharmacol, 2014. 22(2): p. 480-5.

103. Gelati, M., et al., High-dose methylprednisolone reduces cytokine-induced adhesion molecules on human brain endothelium. Can J Neurol Sci, 2000. 27(3): p. 241-4.

104. Dufour, A., et al., Modulation of ICAM-1, VCAM-1 and HLA-DR by cytokines and steroids on HUVECs and human brain endothelial cells. J Neurol Sci, 1998. 157(2): p. 117-21.

105. Hartmann, C., et al., TIMP-3: a novel target for glucocorticoid signaling at the blood-brain barrier. Biochem Biophys Res Commun, 2009. 390(2): p. 182-6. 
106. McCarthy, K.M., et al., Occludin is a functional component of the tight junction. J Cell Sci, 1996. 109 ( Pt 9): p. 2287-98.

107. Harke, N., et al., Glucocorticoids regulate the human occludin gene through a single imperfect palindromic glucocorticoid response element. Mol Cell Endocrinol, 2008. 295(1-2): p. 39-47.

108. Tsukita, S., M. Furuse, and M. Itoh, Multifunctional strands in tight junctions. Nat Rev Mol Cell Biol, 2001. 2(4): p. 285-93.

109. Wolburg, H. and A. Lippoldt, Tight junctions of the blood-brain barrier: development, composition and regulation. Vascul Pharmacol, 2002. 38(6): p. 323-37.

110. Krause, G., et al., Structure and function of claudins. Biochim Biophys Acta, 2008. 1778(3): p. 631-45.

111. Burek, M. and C.Y. Forster, Cloning and characterization of the murine claudin-5 promoter. Mol Cell Endocrinol, 2009. 298(1-2): p. 19-24.

112. Forster, C., et al., Glucocorticoid effects on mouse microvascular endothelial barrier permeability are brain specific. J Physiol, 2006. 573(Pt 2): p. 413-25.

113. Adams, S.E. and G. Melnykovych, Synergistic stimulation of alkaline phosphatase activity in bovine aortic endothelial cells grown in the presence of retinoids and glucocorticoids. J Cell Physiol, 1985. 124(1): p. 120-4.

114. Salvador, E., S. Shityakov, and C. Forster, Glucocorticoids and endothelial cell barrier function. Cell Tissue Res, 2014. 355(3): p. 597-605.

115. Beuckmann, C., S. Hellwig, and H.J. Galla, Induction of the blood/brain-barrierassociated enzyme alkaline phosphatase in endothelial cells from cerebral capillaries is mediated via cAMP. Eur J Biochem, 1995. 229(3): p. 641-4.

116. Roux, F., et al., Regulation of gamma-glutamyl transpeptidase and alkaline phosphatase activities in immortalized rat brain microvessel endothelial cells. J Cell Physiol, 1994. 159(1): p. 101-13.

117. Nakazato, H., et al., Alkaline phosphatase expression in cultured endothelial cells of aorta and brain microvessels: induction by interleukin-6-type cytokines and suppression by transforming growth factor betas. Life Sci, 1997. 61(20): p. 2065-72. 